\title{
SUPPLEMENTARY MATERIAL: Detrital Zircon U-Pb Provenance of the Colorado River: A Five Million Year Record of Incision into Cover Strata Overlying the Colorado Plateau and Adjacent Regions
}

David L. Kimbrough ${ }^{1}$, Marty Grove ${ }^{2}$, George E. Gehrels ${ }^{3}$, Rebecca J.

Dorsey $^{4}$, Keith A. Howard ${ }^{5}$, Oscar Lovera ${ }^{6}$, Andres Aslan ${ }^{7}$, P. Kyle House ${ }^{8}$, Philip A. Pearthree ${ }^{9}$

${ }^{1}$ Department of Geological Sciences, San Diego State University, San Diego, CA 92182

${ }^{2}$ Geological \& Environmental Sciences, Stanford University, Stanford, CA 94305

${ }^{3}$ Department of Geosciences, University of Arizona, Tucson, AZ 85721

${ }^{4}$ Department of Geological Sciences, University of Oregon, Eugene, OR 97403-1272

${ }^{5}$ United States Geological Survey, Menlo Park, CA 94025-3591

${ }^{6}$ Department of Earth and Space Sciences, University of California, Los Angeles, CA 90095

${ }^{7}$ Colorado Mesa University, Grand Junction, CO 81501

${ }^{8}$ United States Geological Survey, Flagstaff, AZ 86001

${ }^{9}$ Arizona Geological Survey, 416 W. Congress St. \#100, Tucson, AZ 85701

\section{SUMMARY OF DATA RESPOSITORY CONTENT}

1. SAMPLE DETAILS. Table DR1. Location and description of U-Pb zircon samples analyzed for this study.

2. LA-ICP-MS METHODS. Description of analytical methods associated with zircon U-Th-Pb laser ablation inductively coupled plasma mass spectrometry (LA-ICP-MS).

3. KOLMOGOROV-SMIRNOV (K-S) STATISTIC. Description of statistical comparisons based upon the Kolmogorov-Smirnov (K-S) statistic.

4. K-S STATISTICS FOR COLORADO RIVER SAMPLES. Tables DR2-6. K-S test comparisons for sandstone and Holocene sand samples from Colorado River basin and associated samples.

5. GENERALIZING THE K-S TEST TO MIXTURES. Explanation of how the K-S statistic is generalized for mixtures.

6. U-PB ZIRCON RESULTS. Table DR7. LA-ICP-MS U-Pb zircon geochronologic analysis results of Colorado River basin and associated samples.

8. U-PB ZIRCON RESULTS FOR SECONDARY STANDARD 49127. Table DR8-9. 
Kimbrough, D.L., Grove, M., Gehrels, G.E., Dorsey, R.J., Howard, K.A., Lovera, O., Aslan, A., House, P.K and Pearthree, P.A

2015, Detrital zircon U-Pb provenance of the Colorado River: A 5 m.y. record of incision into cover strata overlying the Colorado Plateau and adjacent regions: Geosphere, v. 11, doi:10.1130/GES00982.1.

MODERN RIVER SAND

01 CR05-1 $107 \quad 32.2353$

03 1-23-06-1

04 Yuma

05 San Felipito

06 Santa Clara

07 CR-1

08 CR-2

09 CR-3

10 CRT0806-1

11 CRT0806-3

12 CRT0806-2

12 CRT0806-2

13 CRT0806-4

15 CRT0806-6

16 CRT0806-7

17 CRT0806-8

18 CRT0806-9

19 32406-1

\subsection{3}

$-115.0553$ §Age \#Collect

20 CRT0806-10

$02 \quad 32.4943$

32.7293

$-114.8149$

$-114.6409$

$-114.6152$

$\begin{array}{ll}32.2376 & -115.0556 \\ 32.1055 & -114.9488\end{array}$

$36.2613-111.8269$

$36.0443-111.9187$

$36.2295-112.3388$

$39.1470-108.7442$

$38.6035-109.5760$

39.1152

$-109.5760$

-110.1098
-109.6178

$\begin{array}{ll}37.2596 & -109.6178 \\ 37.1501 & -109.8659\end{array}$

Colorado River

$-110.6532$

Colorado River

Colorado River

Colorado River

Colorado River

Colorado River

Grand Canyon

Grand Canyon

Grand Canyon

"Grand" River

"Grand" River

Green River

San Juan River
San Juan River

Sittle Colorado River

MIO-PLIO BIDAHOCHI BASIN NE AZ

54

35.1697

$-109.4383$

$-109.3620$

Bidahochi Fm

LOWER COLORADO RIVER SAND

$\begin{array}{llrr}23 & 32706-3 & 58 & 34.1613 \\ 24 & 32306-175 & 53 & 35.3781\end{array}$

$25 \quad 32506-1$

$33 \quad 35.0871$

$-114.3005$

$-114.5904$

Sub-Bouse

Lost Cabin beds

Bouse Fm

$26 \quad 32103-1$

$71 \quad 35.1458$

$-114.5774$

Bullhead alluvium

$27 \quad 320013-13$

$69 \quad 35.3422$

$-114.5883$

Bullhead alluvium

Bouse Fm

$29 \quad 06322-6$

$\begin{array}{ll}30 & 06322-37\end{array}$

35.3536

$-114.539$

Bullhead alluvium

Bullhead alluvium

Chemehuevi Fm

32 1-22-06-2

34.8901

35.2256

undifferentiated

-114.0601 Muddy Creek Fm

modern

modern

modern

modern

modern

modern

modern

modern

modern

modern

modern

modern

moder

moder

moder

modern

modern

modern

modern

modern

modern

Miocene-Pliocene

Miocene-Pliocene

33 32606-1 51

36.0747 late Miocene

$\sim 5.5 \mathrm{Ma}$

$\sim 5.5 \mathrm{Ma}$

early Pliocene

early Pliocene

late Miocene

early Pliocene

early Pliocene

late Pleistocene

Irvingtonian

late Miocene
DK San Felipito railroad bridge - east side of river - mix of 4 buckets of sand collected from bridge to $\sim 2 \mathrm{~km}$ south of bridge

K Mx Hwy 2 bridge crossing Colorado River San Luis RC - mix of 3 different buckets

Winterhaven - Rivers Edge RV resort

KF Yuma bridge

San Felipito railroad bridge - west side of river

KF Cienega de Santa Clara

GG Kwagunt Creek - $8.3 \mathrm{~km}$ upstream from confluence with Little Colorado River

Hance rapids - 150 meter upstream from rapids at boat pullout

Bass rapids - beach 150 meters upstream of rapids

Fruita, CO; Colorado River State Park

sand bar just upstream from St Hwy 191 bridge crossing

Lower Grey Canyon Rec site

Sand Island UT - St Hwy 191 mile 22 boat launch \& campground near Sand Island Petroglyphs

DK Mexican Hat - $\mathrm{N}$ side of river upstream from St Hwy 163 bridge

DK sample from $\mathrm{S}$ bank of river $\sim 50 \mathrm{~S}$ of $\mathrm{I}-87$ bridge crossing; floodplain terrace $\sim 2 \mathrm{~m}$ above river bottom

DK sample from S bank of river $\sim 500 \mathrm{~m}$ upstream of hwy 89 - floodplain terrace deposit $\sim 1 \mathrm{~m}$ above riverbed w/ tamarisk established on surface

DK sample from $\mathrm{N}$ bank of river $\sim 50 \mathrm{~m} \mathrm{~W}$ of gravel road crossing - floodplain deposit $\sim 2 \mathrm{~m}$ above river bottom $\mathrm{w} /$ tamarisk growth

DK sample from $\mathrm{N}$ bank of river $\sim 100 \mathrm{~m} \mathrm{E}$ of road - floodplain deposit $\mathrm{w} /$ tamarisk $\sim 1-2 \mathrm{~m}$ above riverbottom

DK sand sample from beneath the bypassed McPhaul suspension bridge $\sim 1 / 4$ mile west of where Hwy 95 crosses Gila River bed, $\sim 20$ east of Yuma - clean well sorted sand from "beach" next to flowing Gila R channe

DK sample collected $\sim 700 \mathrm{~m}$ downstream from Riverside bridge crossing; from $\sim 2 \mathrm{~m}$ high terrace next to river; laminated \& cross-bedded fluvial ss pebbly ss - tamarisk well established

DK upper member Bidahochi Fm; sample from "La Pinta Mesa Road" roadcut off of hwy191 $~ 7$ miles N of I-40; $~ 2 \mathrm{~m}$ high roadcut in reddish fluvial ss w/ faint laminations

DK upper member Bidahochi Fm; sample from "Chambers Community Road" roadcut off of hwy $191 \sim 2$ miles South from I40; poorly indurated reddish fluvial ss and pebbly ss w/ many root casts - thin silty interlayers - lower/upper Bidahochi contact well exposed to west of hwy191 to north of here
corly ind

JRM Parker Bridge - friable ss - sub-Bouse fluvial sediments

PAP Lost Cabin beds - stratigraphic equivalent of Muddy Creek Fm. sand sample $\sim 30 \mathrm{~m}$ below 3rd tephra, Spirit Mtn NE quad, Cottonwood Valley

DK\&KHs\& Bouse sand from Silver Creek wash outcrop - highest elevation Bouse in Mojave Valley; possibly transient high level

PAP lake stand - Bouse here is $\sim 3 \mathrm{~m}$ thick with lower and upper shaly and sandstones; sample from $\sim 15 \mathrm{~cm}$ thick sand near top of section

KHs Coarse sand from near the base of the early Colorado River section in Northern Mohave Valley--this is the strat. lowest sample

KHs Fine sand near the lowest exposure of early Colorado River sediments in Cottonwood Valley

KHs 'Colorado River-like' sand in the Bouse Formation in Cottonwood Valley (sand interbedded with green clay typical of Bouse, section underlain by basal marl)

PAP sand $840 \mathrm{ft}$ asl, Boundary Cone quad, Mohave Valley

PAP sand $1220 \mathrm{ft}$ asl, Boundary Cone quad, Mohave Valley

DK\&KHd Chemehuevi "unit E" sand facies of Metzger at top of aggradational package; loose sand $\sim 4 \mathrm{~m}$ above of $\mathrm{D}$ facies; $-50,000$ years old

RD lower to mid-Irvingtonian - El Golfo de Santa Clara / Upper Trash Canyon

DK\&KHd Muddy Creek sandstone bed collected from Pearce Ferry road outcrop beneath Grapevine Mesa \& "airport overlook" of Grand Wash; $15 \mathrm{~cm}$ thick fluvial ss bed interbedded w/ reddish shale $\sim 30$ meters beneath the upper Miocene Hualapai Limestone 
Kimbrough, D. L, Grove, M., Gehrels, G.E Dorsey, R.J., Howard, K.A Lovera, O Aslan, A , House, P.K, and Pearthree, P.A

2015, Detrital zircon U-Pb provenance of the Colorado River: A 5 m.y. record of incision into cover strata overlying the Colorado

Plateau and adjacent regions: Geosphere, v. 11, doi:10.1130/GES00982.1.

TABLE DR1. COLORADO RIVER DETRITAL ZIRCON SAMPLE DETAILS

\begin{tabular}{|c|c|c|c|c|c|c|c|c|}
\hline$\overline{\mathrm{ID} \#}$ & Sample & ${ }^{*} \mathrm{~N}$ & 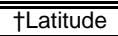 & Longitude & Formation/unit & §Age & \#Collect & Details \\
\hline 34 & H5HW-21 & 139 & 35.9177 & -114.1813 & Hualapai Wash & Miocene-Pliocene & $\mathrm{KHd}$ & sent to DK in mail by KH May 2006 to replace LM-HW-1 \\
\hline 35 & LMSP2 & 143 & 36.1156 & -114.1103 & Sandy Point & early Pliocene & $\mathrm{KHd}$ & gravels underneath 4.4 Ma basalt @ Sandy Point in Lk Meade \\
\hline \multirow[t]{2}{*}{36} & DM305 & 55 & 36.0878 & -114.1015 & Chemehuevi Fm & late Pleistocene & DM & South Cove TL sample; unit D; $2 / 20 / 06$ late Pleistocene $\sim 50,000$ years \\
\hline & WESTERN & TON & OUGH & & & & & \\
\hline 37 & FC06-3 & 58 & 32.9951 & -116.1181 & Imperial Group & $5.33 \mathrm{Ma}$ & DK & $\begin{array}{l}\text { 04Pw21; biot qtzofeldspathic ss - }-0.5 \mathrm{~m} \text { thick massive bed within well-bedded outcrop sequence at edge of wash - } \\
\sim 10 \mathrm{~m} \text { stratigraphically benearth incoming of CR sand }\end{array}$ \\
\hline 38 & FC05-1 & 86 & 32.9943 & -116.1191 & $\begin{array}{l}\text { Wind Caves } \\
\text { member }\end{array}$ & $5.29 \mathrm{Ma}$ & DK & $\begin{array}{l}\text { 04Pw25; Imperial Group - Latrania Fm - Wind Caves member; sandstone from } ~ 1.5 \mathrm{~m} \text { thick bed paleomag site } 04 \mathrm{Pw} 25 \\
\text { Fish Creek wash Split Mtn - above second sturstrom - within a few } 10 \mathrm{~s} \text { of meters of abrupt transition from L-suite (La } \\
\text { Posta sandstone) to C-suite (Colorado River) sandstone }\end{array}$ \\
\hline 39 & FC12-6 & 66 & 32.9940 & -116.1195 & $\begin{array}{l}\text { Wind Caves } \\
\text { member }\end{array}$ & $5.26 \mathrm{Ma}$ & $\mathrm{RD}$ & 04Pw27; Imperial Group - Latrania Fm - Wind Caves member \\
\hline 40 & FC05-2 & 57 & 32.9940 & -116.1196 & $\begin{array}{l}\text { Wind Caves } \\
\text { member }\end{array}$ & $5.26 \mathrm{Ma}$ & DK & $\begin{array}{l}\text { 04Pw27; Imperial Group - Latrania Fm - Wind Caves member; } 3 \text { meter thick amalgamated sandstone bed - paleomag } \\
\text { site 04Pw27 upsection } 20 \text { meters from FC05-1 }\end{array}$ \\
\hline 41 & FC12-7 & 68 & 32.9941 & -116.1202 & $\begin{array}{l}\text { Wind Caves } \\
\text { member }\end{array}$ & $5.24 \mathrm{Ma}$ & $\mathrm{RD}$ & between 04Pw28 and 04Pw29; Imperial Group - Latrania Fm - Wind Caves member \\
\hline 42 & FC05-3 & 124 & 32.9855 & -116.1271 & Mud Hill Member & 4.8 Ma & DK & $\begin{array}{l}\text { 04Pm32; Imperial Group - Deguynos Fm - Mud Hill Member - sandstone from rhymically bedded pro-delta deposits - } \\
\text { within the Sidufjall nomal polarity interval paleomag site } ~-04 \mathrm{Pm} 48\end{array}$ \\
\hline 43 & FC06-1 & 117 & 32.9748 & -116.1625 & Diablo Formation & 4.2 Ma & DK\&RD & $\begin{array}{l}\text { 02Tpd9; Palm Spring Group - Diablo Formation - sandstone Fish Creek Wash } \sim 2 \text { meter thick massive sandstone - from } \\
\text { sandy base of fluvial Diablo facies - massive 1-2 } \mathrm{m} \text { channel fill sands with thinner calcite cemented laminated ss - } \\
\text { within the Cochiti normal polarity interval }\end{array}$ \\
\hline 44 & FC06-2 & 114 & 32.9746 & -116.2072 & Diablo or Olla? Fm & 3.65 Ma & DK\&RD & $\begin{array}{l}\text { 02Tpd18; Palm Spring Group - Diablo or Olla? }-2 \mathrm{~m} \text { thick sandstone from massive trough } \mathrm{x} \text {-bedded fluvial channel } \\
\text { facies sandstone } \sim 30 \mathrm{~m} \text { below Gilbert-Gauss reversal - pure L-suite sand beds occur above and below this interval }\end{array}$ \\
\hline 45 & $2-4-06-1$ & 124 & 33.2344 & -116.2252 & upper Diablo Fm & $3.1 \mathrm{Ma}$ & $\mathrm{RD}$ & equivalent to 04Pd12; upper Diablo Fm, southern Borrego badlands \\
\hline 46 & $2-4-06-2$ & 117 & 33.2402 & -116.2221 & lower Borrego Fm & $2.4 \mathrm{Ma}$ & $\mathrm{RD}$ & equivalent to 04QTb30; "C-ss" above S.1 sst lower Borrego Fm, southern Borrego badlands \\
\hline \multirow[t]{2}{*}{47} & $2-4-06-3$ & 126 & 33.2467 & -116.2239 & upper Borrego Fm & $1.3 \mathrm{Ma}$ & $\mathrm{RD}$ & $\begin{array}{l}\text { between 04QTb49 and 04QTb50; 1-m sst below S6; } 152 \mathrm{~m} \text { below base of Ocotillo Fm, which is } 1.05 \text { +/- .03 Ma; } \sim 1.2 \\
\text { Ma - upper Borrego Fm, southern Borrego badlands }\end{array}$ \\
\hline & BROWNS & $\mathrm{FOR}$ & ATION & & & & & \\
\hline 48 & Tbp81412 & 95 & 40.12077 & -107.16699 & Browns Park Fm & late Miocene & DN & $\begin{array}{l}\text { Pyramid Peak, CO; fluvial sandstone outcrop; pebbly sand in uppermost Tbp exposure between upper and lower basalt } \\
\text { flows on Pyramid Pk; collected by Doug Nichols }\end{array}$ \\
\hline 49 & Tbp7912-2 & 92 & 40.64819 & -108.55599 & Browns Park Fm & late Miocene & AA & $\begin{array}{l}\text { John Weller Mesa, CO; Tbp fluvial sandstone outcrop poorly exposed along NE side of main hwy; outcrop higher } \\
\text { topographically than the } 8.6 \text { Ma ash from E. Boone Draw indicating a young Tbp age }\end{array}$ \\
\hline
\end{tabular}

${ }^{*} \mathrm{~N}=$ number of zircon analyses from sample

$\dagger$ WGS84 datum

$\S$ Western Salton Trough sample ages determined by linear interpolation between magnetic reversals (Dorsey et al., $2011 \mathrm{GSAB}$ )

\# Collect = DK, Kimbrough, RD, Flessa, GG, Gehrels, JRM, Morgan, PAP, Pearthree, KHd, House, RD, Dorsey, DN, Doug Nichols, AA, Aslan 
Kimbrough, D.L., Grove, M., Gehrels, G.E., Dorsey, R.J., Howard, K.A., Lovera, O., Aslan, A., House, P.K., and Pearthree, P.A., 2015, Detrital zircon U-Pb provenance of the Colorado River: A 5 m.y. record of incision into cover strata overlying the Colorado Plateau and adjacent regions: Geosphere, v. 11, doi:10.1130/GES00982.1.

\section{LA-ICP-MS METHODS}

3758 detrital zircon U-Pb ages from 49 samples are reported here. Detrital zircons were separated from modern river sand and sandstone samples by standard crushing, density and magnetic separation techniques at San Diego State University. Sample locations and details are presented in Table 3 of the paper. With the exception of a few gravelly sandstone samples from the Bullhead Alluvium, samples typically were fine- to medium grained moderately well-sorted subarkosic sand and sandstone. Zircon concentrates were mounted together with a Sri Lanka zircon standard SL-2 (564 Ma) or SL-Marty (557 Ma) reference zircon. Sample 41927 was used as a secondary standard on all mounts (137 Ma). Zircons potted in epoxy, sectioned to expose the outer $\sim 1 / 3$ of the average grain dimension, and lightly polished.

Zircons were analyzed during seven different sessions at the University of Arizona LaserChron Center (Gillis et al., 2005; Gehrels et al., 2006, 2008; Johnson et al., 2009). Samples analyzed prior to February 2009 were analyzed with a Multicollector Inductively Coupled Plasma Mass Spectrometer (GVI Isoprobe); later samples were analyzed with a Nu HR ICPMS. The GVI Isoprobe instrument is equipped with nine faraday collectors, an axial Daly detector, and four ion-counting channels. The mass spectrometer is coupled to a New Wave Instruments Excimer laser ablation system which has an emission wavelength of $193 \mathrm{~nm}$. The laser output energy was $\sim 32 \mathrm{~mJ}$ with a repetition rate of $8 \mathrm{hz}$. Analyses were conducted on 35 microns spots and the depth of each ablation pit was 20 microns. Each analysis consisted of one 20-s integration on backgrounds (on peak centers with no laser firing) and twenty 1-s integrations on peaks with the laser firing. The collector configuration allows simultaneous measurement of ${ }^{204} \mathrm{~Pb}$ in a secondary electron multiplier while ${ }^{206} \mathrm{~Pb},{ }^{207} \mathrm{~Pb},{ }^{208} \mathrm{~Pb},{ }^{232} \mathrm{Th}$, and ${ }^{238} \mathrm{U}$ are measured with Faraday detectors. All analyses were conducted in static mode. Hg contributions to ${ }^{204} \mathrm{~Pb}$ were removed by measuring on peak backgrounds. Inter-element fractionation for both the GVI Isoprobe and Nu HR ICPMS instrument measurements were monitored by reference to the Sri Lanka zircon standards. The standard zircon was analyzed once for every five unknowns. A protocol was established to ensure random selection of sample grains.

The Nu HR ICPMS measures $\mathrm{U}$, Th, and Pb isotopes simultaneously in static mode, using Faraday detectors with $3 \times 10^{11}$ ohm resistors for ${ }^{238} \mathrm{U},{ }^{232} \mathrm{Th},{ }^{208} \mathrm{~Pb},{ }^{208} \mathrm{~Pb},{ }^{207} \mathrm{~Pb},{ }^{206} \mathrm{~Pb}$, and discrete dynode ion counters for ${ }^{204} \mathrm{~Pb}$ and ${ }^{202} \mathrm{Hg}$. Ion yields are $\sim 0.8 \mathrm{mv}$ per $\mathrm{ppm}$. Each analysis consists of one 15-second integration on peaks with the laser off (for backgrounds), 15 one- 
second integrations with the laser firing, and a 30 second delay to purge the previous sample and prepare for the next analysis. The analyses involve ablation of zircon with a New Wave UP193HE Excimer laser (operating at a wavelength of $193 \mathrm{~nm}$ ) The ablation pit is typically 30 microns in diameter and $\sim 15$ microns in depth. For each analysis, the errors in determining ${ }^{206} \mathrm{~Pb} /{ }^{238} \mathrm{U}$ and ${ }^{206} \mathrm{~Pb} /{ }^{204} \mathrm{~Pb}$ result in a measurement error of $\sim 1-2 \%$ (at 2-sigma level) in the ${ }^{206} \mathrm{~Pb} /{ }^{238} \mathrm{U}$ age. The errors in measurement of ${ }^{206} \mathrm{~Pb} /{ }^{207} \mathrm{~Pb}$ and ${ }^{206} \mathrm{~Pb} /{ }^{204} \mathrm{~Pb}$ also result in $\sim 1-2 \%$ (at 2-sigma level) uncertainty in age for grains that are $>1.0 \mathrm{Ga}$, but are substantially larger for younger grains due to low intensity of the 207Pb signal. For most analyses, the cross-over in precision of ${ }^{206} \mathrm{~Pb} /{ }^{238} \mathrm{U}$ and ${ }^{206} \mathrm{~Pb} /{ }^{207} \mathrm{~Pb}$ ages occurs at $\sim 1.0 \mathrm{Ga}$. 204Hg interference with ${ }^{204} \mathrm{~Pb}$ is accounted for measurement of ${ }^{202} \mathrm{Hg}$ during laser ablation and subtraction of ${ }^{204} \mathrm{Hg}$ according to the natural ${ }^{202} \mathrm{Hg} /{ }^{204} \mathrm{Hg}$ of 4.35 . This $\mathrm{Hg}$ is correction is not significant for most analyses because $\mathrm{Hg}$ backgrounds in the $\mathrm{Nu}$ instrument are low (generally 150 cps at mass 204). Common Pb correction is accomplished by using the Hg-corrected ${ }^{204} \mathrm{~Pb}$ and assuming an initial $\mathrm{Pb}$ composition from Stacey and Kramers (1975). Uncertainties of 1.5 for ${ }^{206} \mathrm{~Pb} /{ }^{204} \mathrm{~Pb}$ and 0.3 for ${ }^{207} \mathrm{~Pb} /{ }^{204} \mathrm{~Pb}$ are applied to these compositional values based on the variation in $\mathrm{Pb}$ isotopic composition in modern crystal rocks.

In this study, interpreted ages are generally based on ${ }^{206} \mathrm{~Pb} /{ }^{207} \mathrm{~Pb}$ ratios for grains $>900$ $\mathrm{Ma}$, and ${ }^{206} \mathrm{~Pb} /{ }^{238} \mathrm{U}$ ratios for grains $<900 \mathrm{Ma}$. The analytical data for unknowns and for the 49127 standard are reported in supplementary data tables below. Uncertainties shown in these table are at the 1-sigma level, and include only measurement errors. Relative and cumulative age-probability diagrams were prepared using Origin data analysis and graphing software.

\section{KOLMOGOROV-SMIRNOV (K-S) STATISTIC}

As summarized by Press et al. (2002), the Kolmogorov-Smirnov (K-S) statistic may be used to evaluate the validity of the null hypothesis (i.e., that differences between two distributions are caused by insufficient sampling and/or experimental error). The advantage of the K-S test over statistical measures such as the student's $t$ test is that no assumptions are made with respect to the nature of the distributions being compared (see Press et al., 2002). In order to support extension of the K-S statistic to mixtures presented later, we first outline the basis of the standard two sample K-S test. Evaluation of the null hypothesis is achieved by measuring the absolute value of the maximum difference $(D)$ between two cumulative distribution functions (CDF):

$$
D=\frac{\max }{-\infty<x<\infty}\left|S_{N_{1}}(x)-S_{N_{2}}(x)\right|
$$


where $S_{N_{1}}(x)$ and $S_{N_{2}}(x)$ are different data sets assumed by the null hypothesis to be drawn from the same cumulative distribution function. Provided that the value of $D$ is non-zero, its significance $(P)$ can be assessed from the following function (Press. Et al 2002):

$$
P=2 \sum_{j=1}^{\infty}(-1)^{j-1} e^{-2 j^{2} \lambda^{2}} ; \quad \lambda=\sqrt{N_{e}} D
$$

where $P$ varies from zero to unity. In the two sample test, $N_{e}$ is given by:

$$
N_{e}=\frac{N_{1} N_{2}}{N_{1}+N_{2}}
$$

where $N_{l}$ and $N_{2}$ are the respective sizes of samples 1 and 2. By convention, two distributions are regarded to be distinguished at 95\% confidence (i.e., null hypothesis $=$ false) if the probability $(P)$ that $D$ could be lower than the observed value is less than 0.05 . The sensitivity of the K-S test increases as $N_{e}$ is increased but also depends upon the age resolution with which [1] is evaluated.

In this study, we use only non-error-weighted CDF's to calculate $\mathrm{P}$ values. Weighting measured ages by absolute error artificially increases the $P$ value since $D$ is reduced when calculated from cumulative probability distribution functions (CPDF). Use of CPDF to calculate $D$ biases the calculation toward larger $D$ at the young end of the distribution since older, less precise U-Pb ages are smoothed to a greater extent (e.g., Vermeesch, 2012). Weighting according to error thus causes the K-S test to depend upon the nature of the age distribution. This practice thus negates the value of the K-S test as a statistical measure of non-normal distributions (Press et al., 2002).

The form of [2] is shown in Figure DR1. Modified expressions are required for cases where $N_{\mathrm{e}}$ is small in [3] (Press et al., 2002). The dependence of $D$ and $P$ upon $N_{\mathrm{e}}$ is shown in Figures DR1B. Solutions of [2] are shown for $N_{\mathrm{e}}$ values of 9, 25, 100, 400, and 900 respectively. As indicated, the $P$ value associated with a given $D$ is a sensitive function of $N_{\mathrm{e}}$. This is best illustrated by considering the locus of values that correspond to the $95 \%$ confidence threshold (i.e., $P=0.05$ ) represented by dashed line labeled $D_{\text {crit. }}$ The values of $D_{\text {crit }}$ are plotted as a function of $N_{\mathrm{e}}^{0.5}$ in Figure DR1C. For very small sample sizes (i.e., $N_{\mathrm{e}}=9$ ), the value of $D$ corresponding to $\mathrm{P}=0.05$ is large $\left(D_{\text {crit }}=0.435\right)$ and the $\mathrm{K}-\mathrm{S}$ test is quite insensitive. As $N_{\mathrm{e}}$ is increased, $D_{\text {crit }}$ is reduced to small values. For example, values of $N_{\mathrm{e}}>185$ are required to achieve $D_{\text {crit }}<0.10$. Doubling $N_{\mathrm{e}}$ to 370 only reduces $D_{\text {crit }}$ to 0.07 . The value of $N_{\mathrm{e}}$ must be doubled again to 740 to 
Kimbrough, D.L., Grove, M., Gehrels, G.E., Dorsey, R.J., Howard, K.A., Lovera, O., Aslan, A., House, P.K., and Pearthree, P.A., 2015, Detrital zircon U-Pb provenance of the Colorado River: A 5 m.y. record of incision into cover strata overlying the Colorado Plateau and adjacent regions: Geosphere, v. 11, doi:10.1130/GES00982.1.

achieve $D_{\text {crit }}$ of 0.05 . Hence the increasing $N_{\mathrm{e}}$ increases the sensitivity of the K-S test but with progressively diminishing returns (Fig. DR1C).

\section{K-S STATISTICS FOR COLORADO RIVER SAMPLES}

K-S statistical results for Colorado River samples are presented in Tables DR2-6. All use age distributions that have not been weighted for analytical uncertainty. As indicated in Table DR2, all Holocene sands from the Colorado River delta are indistinguishable at 95\% confidence. The age distributions from all six samples are combined to define the modern Colorado River (MCR) reference.

Table DR3 compares age distributions for major tributaries of the modern Colorado. Also provided are comparisons of the main trunk of the Colorado River near its confluence with the Little Colorado River. With the exception of the Green River, multiple samples exist for each tributary. In all cases, samples from a given tributary yield age distributions that are indistinguishable at 95\% confidence. The Green and San Juan Rivers are indistinguishable from the MCR reference while all other tributaries are distinct in some important way. The Colorado River within the Grand Canyon (at its confluence with the Little Colorado River) is already indistinguishable at 95\% confidence from the MCR reference $(n=601)$.

Tables DR4-5 provide comparisons for early Pliocene sandstones representing the early Pliocene Colorado River. Samples from both the lower Colorado River corridor and the Salton Trough are all indistinguishable from one another. Similarly, pooling results from all lower Colorado River corridor samples and comparing them to the pooled result from the Salton trough demonstrates that the age distributions from both areas are indistinguishable from one another at 95\% confidence $(P=0.32)$. Finally pooling all early Pliocene Colorado River samples to define the PCR reference $(\mathrm{n}=639)$ and comparing this with the MCR reference yields $P=0.03$ and permits us to distinguish the early Pliocene and modern Colorado Rivers at 95\% confidence. The differences that distinguish these distributions are described in the text.

Table DR6 compares the PCR reference with the age distributions of samples that represent important Miocene deposits that are potentially relevant to the evolution of the Colorado River. These include comparisons involving the pooled age distribution for the Muddy Creek formation (Muntean, 2012), two siltstones intercalated with the Hualapai Limestone (Pierce-Lopez et al., 2011), two samples from the Bidahochi Formation (this study), three sandstones deposited by the Crooked Ridge River, and two samples representing the Brown Park 
Formation (this study). Only the Miocene Crooked Ridge River age distribution is indistinguishable from the PCR reference at 95\% confidence.

TABLE DR2. K-S RESULTS FROM LOWERMOST COLORADO RIVER HOLOCENE SANDS

\begin{tabular}{lcccccc}
\hline \hline & $01(\mathrm{n}=107)$ & $02(\mathrm{n}=102)$ & $03(\mathrm{n}=115)$ & $04(\mathrm{n}=94)$ & $05(\mathrm{n}=93)$ & $06(\mathrm{n}=90)$ \\
\hline $01(\mathrm{n}=107)$ & 1.000 & 0.618 & 0.195 & 0.166 & 0.097 & 0.444 \\
$02(\mathrm{n}=102)$ & - & 1.000 & 0.207 & 0.596 & 0.176 & 0.492 \\
$03(\mathrm{n}=115)$ & - & - & 1.000 & 0.847 & 0.903 & 0.237 \\
$04(\mathrm{n}=94)$ & - & - & - & 1.000 & 0.610 & 0.421 \\
$05(\mathrm{n}=93)$ & - & - & - & - & 1.000 & 0.100 \\
$06(\mathrm{n}=90)$ & - & - & - & - & 1.000 \\
\hline
\end{tabular}


Kimbrough, D.L., Grove, M., Gehrels, G.E., Dorsey, R.J., Howard, K.A., Lovera, O., Aslan, A., House, P.K., and Pearthree, P.A., 2015, Detrital zircon U-Pb provenance of the Colorado River: A 5 m.y. record of incision into cover strata overlying the Colorado Plateau and adjacent regions: Geosphere, v. 11, doi:10.1130/GES00982.1.

Table DR3. Colorado River Tributary K-S Results

\begin{tabular}{|c|c|}
\hline "Grand Canyon & 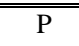 \\
\hline $07(\mathrm{n}=59)$ vs. $08(\mathrm{n}=65)$ & 0.170 \\
\hline $07(\mathrm{n}=59)$ vs. $09(\mathrm{n}=65)$ & 0.566 \\
\hline $08(\mathrm{n}=65)$ vs. $09(\mathrm{n}=65)$ & 0.098 \\
\hline $07(n=59)$ vs. MCR $(n=601)$ & 0.197 \\
\hline $08(n=59)$ vs. MCR $(n=601)$ & 0.184 \\
\hline $09(\mathrm{n}=59)$ vs. MCR $(\mathrm{n}=601)$ & 0.395 \\
\hline Pooled $(n=189)$ vs. MCR $(n=601)$ & 0.201 \\
\hline \multicolumn{2}{|l|}{ Grand River } \\
\hline $10(\mathrm{n}=56)$ vs. $11(\mathrm{n}=53)$ & 0.631 \\
\hline $10(n=56)$ vs. MCR $(n=601)$ & 0.034 \\
\hline $11(n=53)$ vs. MCR $(n=601)$ & 0.025 \\
\hline Pooled $(n=109)$ vs. MCR $(n=601)$ & 0.004 \\
\hline \multicolumn{2}{|l|}{ Green River } \\
\hline $12(n=115)$ vs, MCR $(n=601)$ & 0.939 \\
\hline \multicolumn{2}{|l|}{ San Juan River } \\
\hline $13(\mathrm{n}=55)$ vs. $14(\mathrm{n}=120)$ & 0.515 \\
\hline $13(n=55)$ vs. MCR $(n=601)$ & 0.321 \\
\hline $14(n=120)$ vs. MCR $(n=601)$ & 0.233 \\
\hline Pooled $(n=175)$ vs. MCR $(n=601)$ & 0.140 \\
\hline \multicolumn{2}{|l|}{ Little Colorado River } \\
\hline $15(\mathrm{n}=59)$ vs. $16(\mathrm{n}=64)$ & 0.216 \\
\hline $15(\mathrm{n}=59)$ vs. MCR $(\mathrm{n}=601)$ & $0 . .001$ \\
\hline $16(n=64)$ vs. MCR $(n=601)$ & 0.071 \\
\hline Pooled $(n=123)$ vs. MCR $(n=601)$ & $4 \mathrm{E}-04$ \\
\hline \multicolumn{2}{|l|}{ Gila River } \\
\hline $17(\mathrm{n}=52)$ vs. $18(\mathrm{n}=56)$ & 0.454 \\
\hline $17(\mathrm{n}=52)$ vs. $19(\mathrm{n}=47)$ & 0.651 \\
\hline $18(\mathrm{n}=56)$ vs. $19(\mathrm{n}=47)$ & 0.758 \\
\hline $17(n=52)$ vs MCR $(n=601)$ & 0.003 \\
\hline $18(n=56)$ vs MCR $(n=601)$ & 0.005 \\
\hline $19(n=47)$ vs MCR $(n=601)$ & 0.003 \\
\hline Pooled $(n=155)$ vs. MCR $(n=601)$ & $5 \mathrm{E}-07$ \\
\hline \multicolumn{2}{|l|}{ Virgin River } \\
\hline $20(\mathrm{n}=65)$ vs $08 \mathrm{MC} 20(\mathrm{n}=99)$ & 0.750 \\
\hline $20(\mathrm{n}=65)$ vs. MCR $(\mathrm{n}=601)$ & 0.023 \\
\hline 08MC20 $(n=99)$ vs MCR $(n=601)$ & 0.078 \\
\hline Pooled $(n=164)$ vs MCR $(n=601)$ & 0.003 \\
\hline
\end{tabular}


Kimbrough, D.L., Grove, M., Gehrels, G.E., Dorsey, R.J., Howard, K.A., Lovera, O., Aslan, A., House, P.K., and Pearthree, P.A., 2015, Detrital zircon U-Pb provenance of the Colorado River: A 5 m.y. record of incision into cover strata overlying the Colorado Plateau and adjacent regions: Geosphere, v. 11, doi:10.1130/GES00982.1.

Table DR4. K-S Results for Early Pliocene Colorado River Corridor Sandstones

\begin{tabular}{lccccccc}
\hline \hline & $26(\mathrm{n}=71)$ & $27(\mathrm{n}=69)$ & $28(\mathrm{n}=61)$ & $29(\mathrm{n}=63)$ & $30(\mathrm{n}=59)$ & $34(\mathrm{n}=139)$ & $35(\mathrm{n}=143)$ \\
\hline $26(\mathrm{n}=71)$ & 1.000 & 0.074 & 0.195 & 0.322 & 0.683 & 0.504 & 0.116 \\
$27(\mathrm{n}=69)$ & - & 1.000 & 0.099 & 0.038 & 0.014 & 0.079 & 0.004 \\
$28(\mathrm{n}=61)$ & - & - & 1.000 & 0.464 & 0.160 & 0.704 & 0.129 \\
$29(\mathrm{n}=63)$ & - & - & - & 1.000 & 0.267 & 0.373 & 0.321 \\
$30(\mathrm{n}=59)$ & - & - & - & - & 1.000 & 0.479 & 0.774 \\
$34(\mathrm{n}=56)$ & - & - & - & - & & & 0.480 \\
$35(\mathrm{n}=62)$ & & & & & & & \\
\hline
\end{tabular}

Table DR5. K-S Results for Early Pliocene Salton Trough Sandstones

\begin{tabular}{lcccc}
\hline \hline & $38(\mathrm{n}=86)$ & $39(\mathrm{n}=66)$ & $40(\mathrm{n}=57)$ & $41(\mathrm{n}=68)$ \\
\hline $38(\mathrm{n}=86)$ & 1.000 & 0.292 & 0.340 & 0.196 \\
$39(\mathrm{n}=66)$ & - & 1.000 & 0.856 & 0.716 \\
$40(\mathrm{n}=57)$ & - & - & 1.000 & 0.675 \\
$41(\mathrm{n}=68)$ & - & - & - & 1.000
\end{tabular}

Table DR6. K-S Comparisons for Pliocene Colorado River

\begin{tabular}{lc}
\hline \hline Comparison & P \\
\hline PCR - Lower Colorado River Corridor $(\mathrm{n}=282)$ vs. & 0.647 \\
PCR - Salton Trough $(\mathrm{n}=277)$ & \\
PCR ( $=559)$ vs. & 0.007 \\
MCR ( $\mathrm{n}=601)$ & $1.1 \times 10^{-5}$ \\
PCR ( $=559)$ vs. & \\
Muddy Creek Formation $(\mathrm{n}=1162)$ & $1.2 \times 10^{-5}$ \\
PCR ( $=559)$ vs. & \\
siltstones in Hualapi Limestone $(\mathrm{n}=178)$ & 0.058 \\
PCR ( $=559)$ vs. & \\
Bidahochi Formation $(\mathrm{n}=119)$ & 0.241 \\
PCR ( $=559)$ vs. & \\
Crooked Ridge River $(\mathrm{n}=288)$ & \\
\hline
\end{tabular}

\section{GENERALIZING THE K-S TEST TO MIXTURES}

The K-S test was developed only for comparison of single distributions and no established protocol exists for comparing distributions consisting of pooled data. However, in detrital zircon provenance analysis of systems such as the Colorado River, there is a need for a statistically valid measure that evaluates how different detrital zircon age distributions could have been mixed. Let $X_{1}, X_{2} \ldots X_{\mathrm{m}}$ represent the CDF's measured from the mixing components. The sample sizes and weighting coefficients associated with these individual samples are $n_{1}, n_{2} \ldots n_{\mathrm{m}}$, and $\phi_{1}, \phi_{2} \ldots \phi_{m}$ respectively. For rivers, values of $\phi$ could be considered to represent the proportional contribution 
of each tributary as estimated by catchment area, discharge, bed load, etc. The CDF of the mixture $\left(\mathrm{X}_{\mathrm{w}}\right)$ is defined as:

$$
X_{w}=\sum_{i=1}^{m} \phi_{i}^{*} X_{i} ; \quad \text { where } \sum_{i=1}^{m} \phi_{i}=1 \text { and } N=\sum_{i=1}^{m} n_{i}
$$

where $N$ is the total number of measurements. The mixture, $X_{\mathrm{w}}$, can be assumed to be a linear combination of the individual populations. Applying [2], we can calculate the parameter $D$ to evaluate the null hypothesis:

$$
D=\frac{\max }{-\infty<x<\infty}\left|X_{w}(x)-X_{b}(x)\right|
$$

where $X_{b}$ is a random sample from the mixed age distribution. Because $X_{w}(\mathrm{x})$ depends both upon the nature of the distributions being mixed and the weighting coefficients ( $\left.\phi_{i}\right)$ used to calculate the mixture, the significance function for $D$ will no longer be independent of the distributions being compared. However, there are two cases in which the added degrees of freedom associated with mixtures can be evaluated analytically.

\section{Limiting Cases}

In the development that follows, we define identical and independent populations in terms of the coefficient $r_{\mathrm{ij}}$ between the populations $F_{\mathrm{i}}$ and $F_{\mathrm{j}}$ :

$$
r_{i j}=\frac{\int F_{i} F_{j}}{\sqrt{\int F_{i}^{2} \int F_{j}^{2}}}
$$

Specifically, we define populations $F_{\mathrm{i}}$ and $F_{\mathrm{j}}$ to be identical if $r_{i j}=1 \quad \forall i, j$ and independent if $r_{i j}=0$ $\forall i \neq j$ in [6] above.

Mixtures Drawn from Identical Populations A trivial though important limiting case exists for the situation in which the CDF's of all of the individual data sets $\left(X_{i}\right)$ used to form the mixture were drawn from identical populations. In this special case, the significance of $D$ obtained from [5] can still be calculated from [2] as indicated in Figure DR1A. The form of the curve labeled identical in Figure DR1A is corresponds to that of the two sample K-S test for $N_{\mathrm{e}}=100$. While [3] is still used to calculate $N_{\mathrm{e}}$, the effective size of the mixture $\left(N_{\text {eff }}\right)$ is calculated from the sizes $\left(n_{\mathrm{i}}\right)$ and contributions $\left(\phi_{i}\right)$ of the individual samples as follows:

$$
\frac{1}{N_{e f f}}=\sum_{i=1}^{m} \frac{\phi_{i}^{2}}{n_{i}}
$$


Equation [7] is akin to calculation of the variance of a linear combination of independent and identically distributed random variables (Canavos, 1984).

As discussed above, the precision of the K-S test depends upon the critical value of $D\left(D_{\text {crit }}\right)$ that corresponds to $P=0.05$ depends upon $N_{\mathrm{e}}$. While these are general issues for the K-S test, there are specific issues that relate to how $N_{\text {eff }}$ of the mixture is defined in [7]. The highest $N_{\text {eff }}$ value (i.e. $N_{\text {eff }}=\mathrm{N}$ ) is obtained when $\phi_{i}=\frac{n_{i}}{N}$. Note that the resulting CPDF, $X_{w}=\sum \frac{n_{i}}{N} X_{i}$, is equivalent to merging all of the analysis into a single sample. Alternatively, if $n_{\text {i }}$ is very large relative to $\phi_{\mathrm{i}}$, the effect of $n_{\mathrm{i}}$ upon the magnitude of $N_{\text {eff }}$ will be small. Montecarlo simulations have corroborated the validity of [7].

Mixtures Drawn from Independent Populations A much different second limiting case is provided by the situation in which the individual populations are completely independent. For this case, $P$ is calculated from the product of the individual $P_{\mathrm{i}}$ associated with each component distribution of the mixture. Thus, equation [2] is replaced by:

$$
1-\operatorname{PROB}\left(D>D_{o b s}\right)=\prod_{i=1}^{m}\left(1-Q_{K S}\left(\lambda_{i}\right)\right) ; \quad \lambda_{i}=\sqrt{n_{i}} \frac{D}{\phi_{i}}
$$

Since $D$ is calculated as the maximum difference, it is the probability $(1-P)$ that is relevant because it contains only the combinations that satisfy the same premises for all of the individual comparisons (intersection probability).

As indicated in Figure DR1, the $P$ vs. $D$ curve associated with “independent populations" is shifted towards smaller $D$ values relative to the curve associated with "identical populations". Monte Carlo simulations that we have performed suggest that $P$ vs. $D$ curves corresponding to intermediate cases (i.e. overlapping, non-identical populations) will be bounded by these two limiting solutions. This relationship can be rationalized in terms of the "degrees of freedom" associated with $D$. Specifically, the possible combinations of the "differences" from individual distributions increase with the degree of overlapping of the populations. The highest degree of freedom is obtained when all distributions are derived from identical populations. Hence, in mixing calculations, the assumption of completely independent age distributions yields the most conservative value of $P$ for a given value of $D$ (Figure DR1).

Figure DR1 Relationship between $D$ and $P$ for mixtures when the Kolmorgorov-Smirnoff statistic is adapted for mixtures. Solutions exist for two end member possibilities. When the age 
Kimbrough, D.L., Grove, M., Gehrels, G.E., Dorsey, R.J., Howard, K.A., Lovera, O., Aslan, A., House, P.K., and Pearthree, P.A., 2015, Detrital zircon U-Pb provenance of the Colorado River: A 5 m.y. record of incision into cover strata overlying the Colorado Plateau and adjacent regions: Geosphere, v. 11, doi:10.1130/GES00982.1.

(A)

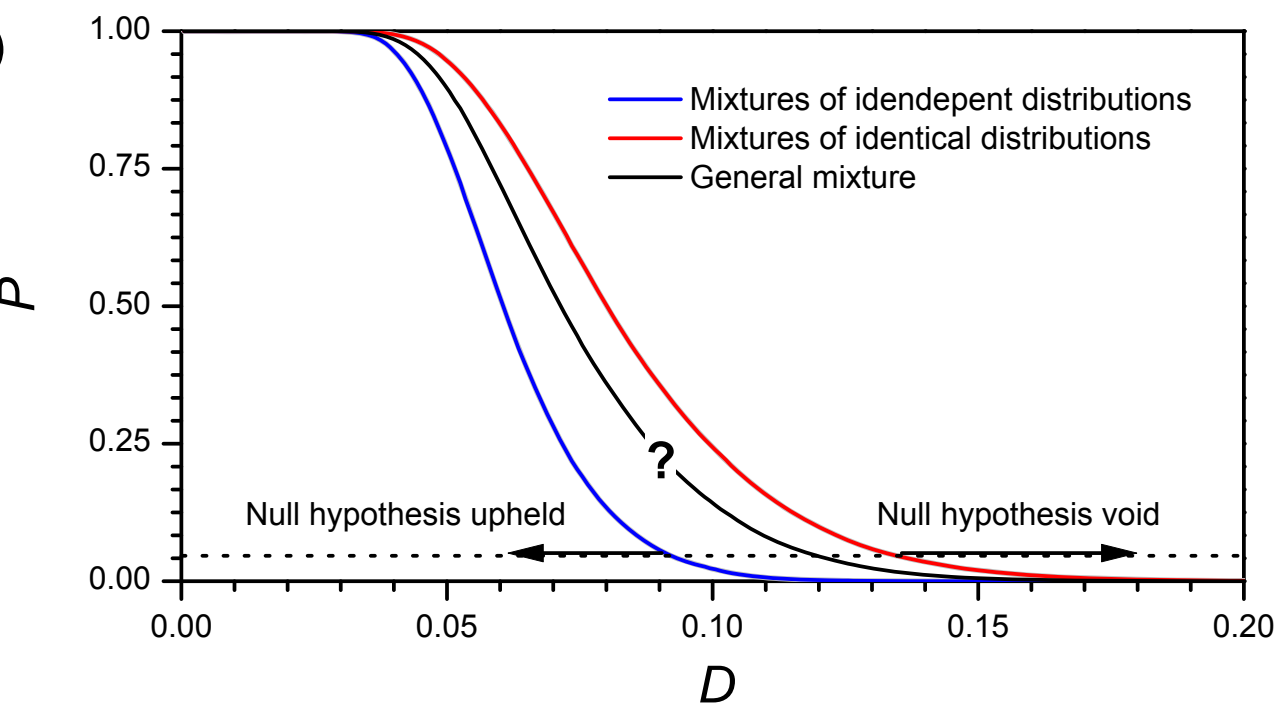

(B)

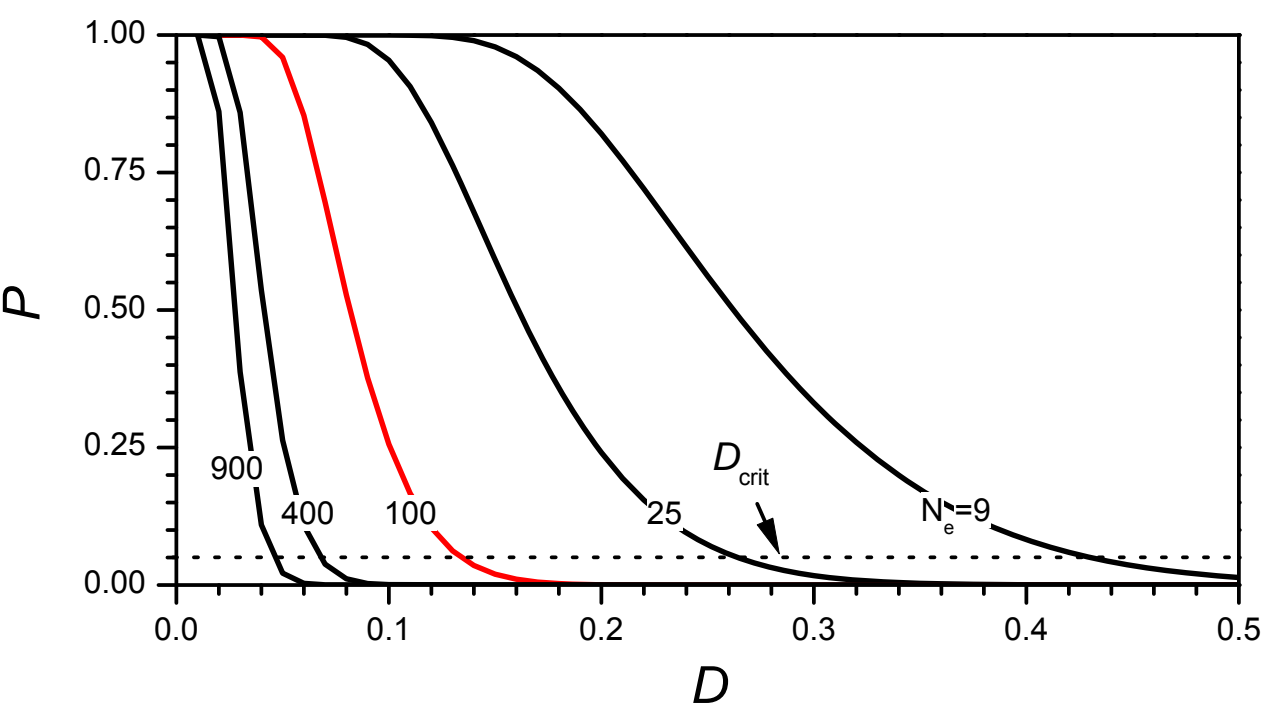

(C)

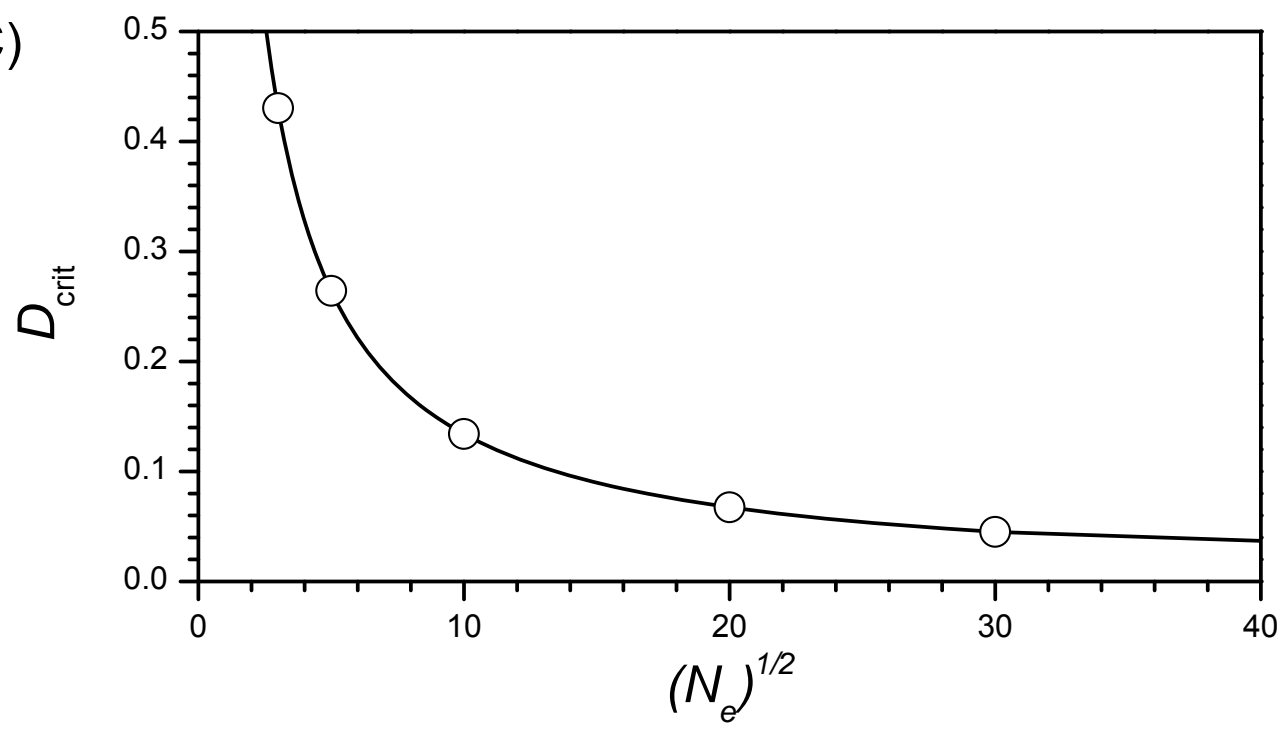


Kimbrough, D.L., Grove, M., Gehrels, G.E., Dorsey, R.J., Howard, K.A., Lovera, O., Aslan, A., House, P.K., and Pearthree, P.A., 2015, Detrital zircon U-Pb provenance of the Colorado River: A 5 m.y. record of incision into cover strata overlying the Colorado Plateau and adjacent regions: Geosphere, v. 11, doi:10.1130/GES00982.1.

distributions being mixed are completely identical, the relationship between $D$ and $P$ is the same as for the conventional K-S statistic with the sample size $N_{\mathrm{e}}$ determined from the weighting coefficients as expressed in [7]. Alternatively, when the distributions being mixed are completely independent, the relationship between $D$ and $P$ is the intersection probability as expressed in [8].

\section{REFERENCES CITED}

Canavos, G.C., 1984, Applied Probability and Statistical Methods: Boston, Little, Brown, 608 p.

Gillis, R.J., Gehrels, G.E., Ruiz, J., and Flores de Dios Gonzaléz, L.A., 2005, Detrital zircon provenance of Cambrian-Ordovician and Carboniferous strata of the Oaxaca terrane, southern Mexico: Sedimentary Geology, v. 182, p. 87-100.

Gehrels, G.E., Valencia, V., Ruiz, J., 2008, Enhanced precision, accuracy, efficiency, and spatial resolution of $\mathrm{U}-\mathrm{Pb}$ ages by laser ablation-multicollector-inductively coupled plasma- mass spectrometry: Geochemistry, Geophysics, Geosystems, v. 9, Q03017, doi:10.1029/2007GC001805.

Gehrels, G.E., Valencia, V., Pullen, A., 2006, Detrital zircon geochronology by Laser-Ablation Multicollector ICPMS at the Arizona LaserChron Center, in Loszewski, T., and Huff, W.,eds., Geochronology: Emerging Opportunities, Paleontology Society Short Course: Paleontology Society Papers, v. 11, 10 p. 38

Ludwig, K.R., 2008, Isoplot 3.60. Berkeley Geochronology Center, Special Publ. No. 4, 77 p.

Johnston, S., Gehrels, G., Valencia, V., and Ruiz, J., 2009, Small-Volume U-Pb Zircon Geochronology by Laser Ablation-Multicollector-ICP-MS: Chemical Geology, v. 259, p. 218-229.

Kimbrough et al., 1992, Uranium-lead ages from the Dun Mountain ophiolite belt and Brook Street Terrane, South Island, New Zealand: Geological Society of America Bulletin, v. 104, p. 429-443. Kimbrough et al.,, 1994, Uranium-lead zircon ages from the Median Tectonic Zone, South Island, New Zealand: New Zealand Journal of Geology and Geophysics, v. 37, p. 393-419.

Mattinson, J.M., Kimbrough, D.L., and Bradshaw, J.D., 1986, Western Fiordland orthogneiss: Early Cretaceous arc magmatism and granulite facies metamorphism, New Zealand: Contribution to Mineralogy and Petrology, v. 92, p. 383-392.

Press, W.H., S. A. Teukolsky, W. T. Vetterling, and B. P. Flannery, editors 2002. Are two distributions different? In Numerical Recipes in C. Cambridge University Press, Cambridge, UK. 620-628.

Stacey, J.S., and Kramers, J.D., 1975, Approximation of terrestrial lead isotope evolution by a two-stage model: Earth and Planetary Science Letters, v. 26, p. 207-221.

Vermeesch, P., 2012, On the visualisation of detrital age distributions: Chemical Geology, v. 312-313, p. 190-194. 
Kimbrough, D.L., Grove, M., Gehrels, G.E., Dorsey, R.J., Howard, K.A., Lovera, O., Aslan, A., House, P.K., and Pearthree, P.A., 2015, Detrital zircon U-Pb provenance of the Colorado River: A 5 m.y. record of incision into cover strata overlying the Colorado Plateau and adjacent regions: Geosphere, v. 11, doi:10.1130/GES00982.1.

TABLE DR7. U-Pb ZIRCON LA-ICP-MS GEOCHRONOLOGIC ANALYSES OF COLORADO RIVER BASIN \& ASSOCIATED SAMPLES.

\begin{tabular}{|c|c|c|c|c|c|c|c|}
\hline & & & & & & opic ra & \\
\hline ID\# & Analysis & $\begin{array}{c}U \\
(\mathrm{ppm})\end{array}$ & $\begin{array}{l}206 \mathrm{~Pb} \\
204 \mathrm{~Pb} \\
\end{array}$ & $\mathrm{U} / \mathrm{Th}$ & $\begin{array}{c}207 \mathrm{~Pb}^{*} \\
235 \mathrm{U}\end{array}$ & $\begin{array}{c} \pm \\
(\%) \\
\end{array}$ & $\begin{array}{c}206 \mathrm{~Pb}^{*} \\
238 \mathrm{U}\end{array}$ \\
\hline 01 & CR05-1-41 & 469 & 737 & 0.2 & 0.03316 & 18.8 & 0.00391 \\
\hline 01 & CR05-1-37 & 793 & 6571 & 0.4 & 0.07485 & 4.5 & 0.01123 \\
\hline 01 & CR051-12 & 801 & 7840 & 3.7 & 0.07680 & 12.2 & 0.01208 \\
\hline 01 & CR05-1-4 & 364 & 4588 & 0.7 & 0.09537 & 5.5 & 0.01422 \\
\hline 01 & CR051-57 & 661 & 6870 & 1.7 & 0.10535 & 9.2 & 0.01489 \\
\hline 01 & CR05-1-9 & 195 & 2415 & 0.9 & 0.11332 & 8.9 & 0.01562 \\
\hline 01 & CR05-1-34 & 298 & 2148 & 0.5 & 0.17112 & 9.1 & 0.02172 \\
\hline 01 & CR051-11 & 70 & 1189 & 1.1 & 0.21023 & 38.7 & 0.02221 \\
\hline 01 & CR05-1-32 & 266 & 3580 & 0.5 & 0.18837 & 5.6 & 0.02589 \\
\hline 01 & CR051-58 & 171 & 2561 & 0.6 & 0.18384 & 14.3 & 0.02606 \\
\hline 01 & CR05-1-39 & 196 & 5240 & 1.3 & 0.20887 & 6.3 & 0.03031 \\
\hline 01 & CR05-1-10 & 194 & 5167 & 0.7 & 0.23104 & 3.9 & 0.03365 \\
\hline 01 & CR051-33 & 459 & 9126 & 0.6 & 0.23114 & 6.0 & 0.03399 \\
\hline 01 & CR05-1-16 & 398 & 10156 & 1.2 & 0.24462 & 5.9 & 0.03453 \\
\hline 01 & CR051-50 & 193 & 5185 & 0.8 & 0.26992 & 8.4 & 0.03651 \\
\hline 01 & CR051-18 & 1101 & 36263 & 1.9 & 0.33071 & 1.5 & 0.04565 \\
\hline 01 & CR051-19 & 275 & 1954 & 3.7 & 0.63272 & 11.9 & 0.06068 \\
\hline 01 & CR051-15 & 422 & 7747 & 2.3 & 0.55301 & 10.9 & 0.06407 \\
\hline 01 & CR051-31 & 476 & 23467 & 0.8 & 0.48035 & 3.6 & 0.06418 \\
\hline 01 & CR05-1-23 & 358 & 11806 & 1.4 & 0.49491 & 3.7 & 0.06424 \\
\hline 01 & CR05-1-43 & 114 & 5699 & 2.2 & 0.51018 & 3.5 & 0.06508 \\
\hline 01 & CR05-1-14 & 916 & 36472 & 2.4 & 0.51084 & 5.4 & 0.06693 \\
\hline 01 & CR051-52 & 208 & 12579 & 1.3 & 0.50044 & 6.4 & 0.06927 \\
\hline 01 & CR051-2 & 316 & 16604 & 1.3 & 0.57116 & 3.7 & 0.07398 \\
\hline 01 & CR05-1-40 & 573 & 20533 & 20.5 & 0.64973 & 7.9 & 0.07532 \\
\hline 01 & CR05-1-8 & 93 & 6691 & 1.0 & 0.74802 & 4.1 & 0.08969 \\
\hline 01 & CR05-1-36 & 296 & 22489 & 1.3 & 0.74903 & 2.8 & 0.09253 \\
\hline & CR051-56 & 48 & 1309 & 1.1 & 0.61399 & 18.1 & 0.09500 \\
\hline 01 & CR051-28 & 228 & 6010 & 2.6 & 0.86109 & 6.7 & 0.09590 \\
\hline 01 & CR051-16 & 169 & 10773 & 1.6 & 0.79318 & 4.0 & 0.09882 \\
\hline 01 & CR051-44 & 65 & 3737 & 1.1 & 0.88220 & 8.9 & 0.09888 \\
\hline 01 & CR05-1-45 & 113 & 7803 & 0.9 & 0.80662 & 2.0 & 0.09893 \\
\hline & CR051-24 & 113 & 9763 & 2.8 & 1.10006 & 4.0 & 0.12647 \\
\hline 01 & CR051-20 & 140 & 14881 & 1.6 & 1.50026 & 3.4 & 0.15579 \\
\hline 01 & CR05-1-28 & 106 & 10728 & 1.9 & 1.53062 & 3.7 & 0.15791 \\
\hline & CR05-1-18 & 58 & 7048 & 1.3 & 1.70752 & 5.4 & 0.17070 \\
\hline 01 & CR051-23 & 452 & 17063 & 1.1 & 1.28406 & 2.8 & 0.12836 \\
\hline 01 & CR05-1-19 & 95 & 14103 & 1.3 & 1.77714 & 3.5 & 0.17364 \\
\hline & CR05-1-42 & 135 & 18143 & 2.1 & 1.76685 & 3.0 & 0.17249 \\
\hline 01 & CR051-37 & 196 & 28798 & 1.8 & 1.76003 & 2.3 & 0.17103 \\
\hline & CR05-1-21 & 31 & 3608 & 0.2 & 1.76375 & 5.4 & 0.17097 \\
\hline 01 & CR051-10 & 329 & 20007 & 1.6 & 1.65130 & 1.6 & 0.15983 \\
\hline 01 & CR051-27 & 164 & 8322 & 2.0 & 1.59214 & 9.2 & 0.15379 \\
\hline & CR051-38 & 298 & 42761 & 2.3 & 1.79952 & 1.9 & 0.17381 \\
\hline 01 & CR05-1-6 & 139 & 17643 & 1.6 & 1.85662 & 1.7 & 0.17917 \\
\hline 01 & CR051-29 & 87 & 9764 & 0.9 & 1.87581 & 4.5 & 0.17885 \\
\hline & CR051-32 & 243 & 33822 & 1.7 & 1.84288 & 2.3 & 0.17485 \\
\hline 01 & CR051-21 & 238 & 24991 & 0.9 & 1.79397 & 2.0 & 0.17017 \\
\hline & CR051-30 & 55 & 1946 & 0.4 & 1.38450 & 7.7 & 0.13060 \\
\hline & CR05-1-22 & 50 & 6205 & 1.1 & 1.95648 & 3.6 & 0.18382 \\
\hline 01 & CR05-1-7 & 88 & 11044 & 0.9 & 2.15005 & 2.2 & 0.19905 \\
\hline & CR05-1-26 & 193 & 19447 & 1.3 & 2.16528 & 2.6 & 0.19913 \\
\hline & CR05-1-48 & 267 & 39629 & 1.8 & 2.27372 & 1.9 & 0.20602 \\
\hline 01 & CR051-9 & 123 & 15920 & 3.0 & 2.23955 & 2.2 & 0.20250 \\
\hline & CR05-1-35 & 786 & 76209 & 3.6 & 2.31209 & 2.4 & 0.20817 \\
\hline & CR05-1-3 & 1007 & 12729 & 1.4 & 1.94209 & 3.2 & 0.17477 \\
\hline 01 & CR05-1-29 & 246 & 22806 & 2.0 & 2.22776 & 2.1 & 0.19975 \\
\hline & CR051-60 & 108 & 9505 & 1.1 & 2.25832 & 3.9 & 0.20010 \\
\hline & CR05-1-50 & 80 & 12659 & 1.3 & 2.43722 & 2.1 & 0.21541 \\
\hline & CR05-1-27 & 177 & 22477 & 2.6 & 2.46362 & 2.5 & 0.21535 \\
\hline & CR05-1-31 & 193 & 24575 & 1.8 & 2.45490 & 2.1 & 0.21453 \\
\hline 01 & CR051-22 & 805 & 12260 & 21.5 & 1.98191 & 8.7 & 0.17303 \\
\hline 01 & CR051-7 & 288 & 7634 & 0.2 & 1.76066 & 3.9 & 0.15366 \\
\hline & CR05-1-17 & 230 & 36631 & 0.9 & 2.63163 & 4.0 & 0.22661 \\
\hline 01 & CR05-1-25 & 261 & 28658 & 0.8 & 2.51405 & 4.4 & 0.21214 \\
\hline & CR051-5 & 99 & 8029 & 1.3 & 2.63534 & 3.0 & 0.22021 \\
\hline & CR051-11A & 221 & 41535 & 0.9 & 2.90996 & 1.4 & 0.23384 \\
\hline 01 & CR051-13 & 124 & 24636 & 0.8 & 3.01201 & 2.8 & 0.24189 \\
\hline 01 & CR051-25 & 94 & 17543 & 0.8 & 3.12645 & 3.5 & 0.24927 \\
\hline & CR051-54 & 381 & 41001 & 2.0 & 2.89712 & 2.5 & 0.23015 \\
\hline & CR051-36 & 402 & 57584 & 2.5 & 3.29706 & 2.3 & 0.26185 \\
\hline 01 & CR051-1 & 66 & 7775 & 0.9 & 3.34182 & 4.8 & 0.26398 \\
\hline 01 & CR051-45 & 217 & 41146 & 1.9 & 3.08526 & 1.3 & 0.24308 \\
\hline & CR051-55 & 29 & 6096 & 0.3 & 3.24022 & 5.6 & 0.25458 \\
\hline & CR05-1-20 & 72 & 15910 & 0.8 & 3.40966 & 3.6 & 0.26619 \\
\hline & CR051-47 & 202 & 42370 & 1.2 & 3.25631 & 2.6 & 0.25333 \\
\hline 01 & CR05-1-12 & 410 & 27303 & 1.1 & 3.35541 & 5.4 & 0.26057 \\
\hline 01 & CR05-1-11 & 870 & 139493 & 1.8 & 3.31893 & 2.0 & 0.25371 \\
\hline & CR051-6 & 337 & 22192 & 2.3 & 2.99390 & 2.7 & 0.22208 \\
\hline 01 & CR05-1-33 & 283 & 32238 & 1.2 & 2.58712 & 2.5 & 0.19157 \\
\hline 01 & CR05-1-46 & 122 & 22323 & 0.9 & 4.06055 & 1.8 & 0.29212 \\
\hline & CR05-1-5 & 448 & 40554 & 2.0 & 3.69744 & 2.4 & 0.26530 \\
\hline & CR051-48 & 114 & 22562 & 1.3 & 3.99479 & 1.1 & 0.28471 \\
\hline & CR051-34 & 234 & 32001 & 1.6 & 3.70297 & 2.7 & 0.26004 \\
\hline & CR05-1-24 & 749 & 91158 & 5.7 & 4.38239 & 3.2 & 0.30680 \\
\hline 01 & CR05-1-1 & 112 & 25450 & 1.0 & 4.29904 & 1.7 & 0.30033 \\
\hline 01 & CR051-4 & 244 & 21512 & 1.4 & 3.64359 & 2.0 & 0.25427 \\
\hline 01 & CR051-8 & 371 & 77007 & 3.7 & 4.49371 & 1.5 & 0.31129 \\
\hline 01 & CR051-46 & 421 & 85828 & 3.6 & 4.43502 & 3.5 & 0.30494 \\
\hline
\end{tabular}


Kimbrough, D.L., Grove, M., Gehrels, G.E., Dorsey, R.J., Howard, K.A., Lovera, O., Aslan, A., House, P.K., and Pearthree, P.A., 2015, Detrital zircon U-Pb provenance of the Colorado River: A 5 m.y. record of incision into cover strata overlying the Colorado Plateau and adjacent regions: Geosphere, v. 11, doi:10.1130/GES00982.1.

TABLE DR7. U-Pb ZIRCON LA-ICP-MS GEOCHRONOLOGIC ANALYSES OF COLORADO RIVER BASIN \& ASSOCIATED SAMPLES.

\begin{tabular}{|c|c|c|c|c|c|c|}
\hline & & & & & & opic ra \\
\hline ID\# & Analysis & $\begin{array}{c}U \\
(\mathrm{ppm})\end{array}$ & $\begin{array}{l}206 \mathrm{~Pb} \\
204 \mathrm{~Pb} \\
\end{array}$ & U/Th & $\begin{array}{c}207 \mathrm{~Pb}^{*} \\
235 \mathrm{U}\end{array}$ & $\begin{array}{c} \pm \\
(\%) \\
\end{array}$ \\
\hline 01 & CR051-53 & 95 & 22536 & 1.3 & 4.34155 & 2.6 \\
\hline 01 & CR05-1-44 & 230 & 33547 & 1.8 & 4.56325 & 2.8 \\
\hline 01 & CR051-14 & 123 & 11278 & 1.5 & 4.57587 & 6.4 \\
\hline 01 & CR051-51 & 222 & 49327 & 1.2 & 4.41207 & 2.0 \\
\hline 01 & CR05-1-13 & 151 & 36318 & 1.5 & 4.83303 & 3.4 \\
\hline 01 & CR051-39 & 755 & 154076 & 1.4 & 4.52800 & 1.0 \\
\hline 01 & CR051-35 & 267 & 49615 & 1.4 & 4.65831 & 1.2 \\
\hline 01 & CR051-40 & 143 & 2076 & 1.0 & 3.89665 & 6.1 \\
\hline 01 & CR051-5A & 192 & 48271 & 1.3 & 5.10229 & 1.1 \\
\hline 01 & CR05-1-47 & 360 & 54403 & 3.6 & 5.77539 & 3.0 \\
\hline 01 & CR05-1-2 & 186 & 49582 & 2.0 & 5.71420 & 3.6 \\
\hline 01 & CR05-1-30 & 172 & 24485 & 1.4 & 5.46862 & 3.3 \\
\hline 01 & CR05-1-49 & 138 & 28656 & 1.2 & 6.18557 & 2.6 \\
\hline 01 & CR051-3 & 122 & 18023 & 1.3 & 6.08182 & 8.7 \\
\hline 01 & CR051-59 & 189 & 45428 & 1.1 & 12.59496 & 1.5 \\
\hline 01 & CR051-43 & 42 & 16912 & 0.5 & 14.40226 & 1.2 \\
\hline 01 & CR05-1-38 & 293 & 105101 & 1.0 & 12.61240 & 3.3 \\
\hline 01 & CR051-42 & 233 & 27974 & 1.3 & 19.02667 & 1.2 \\
\hline 02 & CR-CR052-21 & 322 & 1042 & 0.5 & 0.03676 & 24.9 \\
\hline 02 & CR-CR052-13 & 397 & 1108 & 1.4 & 0.03950 & 16.1 \\
\hline 02 & CR05-2-8 & 1744 & 8751 & 0.5 & 0.06137 & 5.5 \\
\hline 02 & CR05-2-42 & 129 & 964 & 1.4 & 0.08324 & 8.8 \\
\hline 02 & CR-CR052-40 & 99 & 751 & 0.8 & 0.12176 & 44.2 \\
\hline 02 & CR-CR052-41 & 431 & 4378 & 1.2 & 0.09509 & 8.0 \\
\hline 02 & CR05-2-29 & 408 & 9166 & 1.3 & 0.17083 & 4.3 \\
\hline 02 & CR-CR052-34 & 303 & 5286 & 0.9 & 0.19035 & 8.5 \\
\hline 02 & CR-CR052-56 & 619 & 11443 & 0.6 & 0.22309 & 5.5 \\
\hline 02 & CR-CR052-20 & 299 & 7274 & 0.8 & 0.23822 & 5.8 \\
\hline 02 & CR-CR052-35 & 319 & 4365 & 0.8 & 0.26855 & 6.3 \\
\hline 02 & CR05-2-36 & 145 & 1456 & 1.7 & 0.41424 & 17.1 \\
\hline 02 & CR05-2-37 & 1090 & 18875 & 1.0 & 0.27564 & 4.2 \\
\hline 02 & CR05-2-17 & 1200 & 17001 & 0.7 & 0.29279 & 3.2 \\
\hline 02 & CR05-2-28 & 263 & 8521 & 1.4 & 0.31933 & 3.0 \\
\hline 02 & CR-CR052-32 & 223 & 8688 & 0.6 & 0.37008 & 9.7 \\
\hline 02 & CR-CR052-29 & 348 & 11957 & 1.0 & 0.37269 & 4.5 \\
\hline 02 & CR-CR052-8 & 169 & 4987 & 2.3 & 0.40813 & 6.4 \\
\hline 02 & CR05-2-39 & 748 & 27037 & 2.3 & 0.40065 & 2.9 \\
\hline 02 & CR-CR052-33 & 367 & 7608 & 1.4 & 0.46159 & 2.8 \\
\hline 02 & CR05-2-4 & 451 & 14634 & 2.1 & 0.45812 & 4.7 \\
\hline 02 & CR05-2-5 & 377 & 8821 & 0.7 & 0.52408 & 7.7 \\
\hline 02 & CR05-2-27 & 284 & 11218 & 3.2 & 0.48321 & 3.4 \\
\hline 02 & CR05-2-18 & 196 & 13674 & 1.2 & 0.49347 & 3.5 \\
\hline 02 & CR05-2-21 & 255 & 12100 & 1.2 & 0.51762 & 3.5 \\
\hline 02 & CR05-2-12 & 298 & 14720 & 1.1 & 0.50084 & 3.3 \\
\hline 02 & CR-CR052-11 & 166 & 4538 & 1.1 & 0.54415 & 9.3 \\
\hline 02 & CR-CR052-57 & 81 & 4352 & 0.8 & 0.50229 & 15.8 \\
\hline 02 & CR-CR052-7 & 174 & 4146 & 0.4 & 0.67788 & 9.4 \\
\hline 02 & CR-CR052-38 & 390 & 11325 & 2.8 & 0.68573 & 2.6 \\
\hline 02 & CR05-2-22 & 484 & 23092 & 1.0 & 0.67355 & 2.5 \\
\hline 02 & CR-CR052-27 & 560 & 27381 & 10.2 & 0.74034 & 3.5 \\
\hline 02 & CR05-2-20 & 199 & 13446 & 0.7 & 0.76854 & 3.6 \\
\hline 02 & CR05-2-9 & 66 & 4877 & 2.3 & 0.73887 & 4.9 \\
\hline 02 & CR-CR052-51 & 70 & 5341 & 0.7 & 0.77140 & 12.1 \\
\hline 02 & CR05-2-6 & 63 & 2981 & 0.4 & 0.79990 & 5.4 \\
\hline 02 & CR-CR052-43 & 199 & 14477 & 1.9 & 0.80063 & 4.6 \\
\hline 02 & CR-CR052-28 & 331 & 15094 & 0.8 & 0.81142 & 2.5 \\
\hline 02 & CR-CR052-54 & 297 & 14479 & 0.9 & 0.82987 & 3.0 \\
\hline 02 & CR05-2-49 & 61 & 3983 & 0.8 & 0.96508 & 8.1 \\
\hline 02 & CR-CR052-60 & 99 & 9540 & 1.4 & 0.76176 & 4.4 \\
\hline 02 & CR05-2-24 & 305 & 20946 & 0.9 & 0.82940 & 4.2 \\
\hline 02 & CR05-2-45 & 103 & 12226 & 1.1 & 1.75633 & 4.1 \\
\hline 02 & CR-CR052-37 & 223 & 29057 & 1.6 & 1.77510 & 1.3 \\
\hline 02 & CR05-2-450 & 266 & 44925 & 2.1 & 1.75396 & 2.1 \\
\hline 02 & CR05-2-19 & 118 & 22612 & 1.5 & 1.87729 & 3.3 \\
\hline 02 & CR05-2-7 & 331 & 39238 & 2.9 & 1.86945 & 2.5 \\
\hline 02 & CR-CR052-24 & 58 & 6494 & 1.4 & 1.75168 & 4.5 \\
\hline 02 & CR05-2-2 & 245 & 24472 & 1.9 & 1.87436 & 3.3 \\
\hline 02 & CR-CR052-49 & 110 & 12529 & 1.9 & 2.01618 & 4.3 \\
\hline 02 & CR-CR052-2 & 247 & 21124 & 2.6 & 1.88129 & 1.8 \\
\hline 02 & CR05-2-13 & 345 & 26341 & 1.4 & 1.95520 & 3.5 \\
\hline 02 & CR-CR052-15 & 146 & 9956 & 0.8 & 1.84460 & 3.0 \\
\hline 02 & CR-CR052-19 & 122 & 7575 & 2.2 & 1.97341 & 2.1 \\
\hline 02 & CR05-2-14 & 514 & 94781 & 3.3 & 2.13236 & 2.4 \\
\hline 02 & CR05-2-33 & 93 & 16716 & 2.1 & 2.26653 & 2.7 \\
\hline 02 & CR-CR052-39 & 219 & 37447 & 2.3 & 2.18925 & 1.7 \\
\hline 02 & CR-CR052-14 & 193 & 30220 & 1.8 & 2.42250 & 1.3 \\
\hline 02 & CR05-2-15 & 119 & 16154 & 1.0 & 2.04773 & 4.4 \\
\hline 02 & CR-CR052-25 & 142 & 14716 & 2.3 & 2.50508 & 5.4 \\
\hline 02 & CR05-2-31 & 193 & 28656 & 1.7 & 2.81548 & 2.7 \\
\hline 02 & CR-CR052-12 & 221 & 20766 & 3.5 & 2.67182 & 1.4 \\
\hline 02 & CR-CR052-55 & 344 & 59222 & 2.2 & 3.11572 & 1.2 \\
\hline 02 & CR05-2-23 & 216 & 21120 & 1.5 & 3.22549 & 2.8 \\
\hline 02 & CR-CR052-46 & 162 & 20379 & 1.3 & 3.18545 & 6.2 \\
\hline 02 & CR-CR052-58 & 164 & 32865 & 1.7 & 3.33029 & 1.8 \\
\hline 02 & CR05-2-3 & 376 & 27818 & 10.9 & 3.00375 & 2.7 \\
\hline 02 & CR05-2-43 & 235 & 18886 & 1.2 & 2.68512 & 4.2 \\
\hline 02 & CR05-2-46 & 362 & 67072 & 6.3 & 3.72146 & 3.6 \\
\hline 02 & CR-CR052-45 & 110 & 22196 & 1.0 & 3.99089 & 1.7 \\
\hline 02 & CR-CR052-31 & 56 & 13139 & 0.9 & 4.24929 & 2.0 \\
\hline 02 & CR-CR052-4 & 163 & 3794 & 1.7 & 3.37552 & 6.2 \\
\hline
\end{tabular}


Kimbrough, D.L., Grove, M., Gehrels, G.E., Dorsey, R.J., Howard, K.A., Lovera, O., Aslan, A., House, P.K., and Pearthree, P.A., 2015, Detrital zircon U-Pb provenance of the Colorado River: A 5 m.y. record of incision into cover strata overlying the Colorado Plateau and adjacent regions: Geosphere, v. 11, doi:10.1130/GES00982.1.

TABLE DR7. U-Pb ZIRCON LA-ICP-MS GEOCHRONOLOGIC ANALYSES OF COLORADO RIVER BASIN \& ASSOCIATED SAMPLES.

\begin{tabular}{|c|c|c|c|c|c|c|}
\hline & & & & & & opic ra \\
\hline ID\# & Analysis & $\begin{array}{c}\text { U } \\
(\mathrm{ppm})\end{array}$ & $\begin{array}{l}206 \mathrm{~Pb} \\
204 \mathrm{~Pb}\end{array}$ & $\mathrm{U} / \mathrm{Th}$ & $\begin{array}{c}207 \mathrm{~Pb}^{*} \\
235 \mathrm{U}\end{array}$ & $\begin{array}{c} \pm \\
(\%) \\
\end{array}$ \\
\hline 02 & CR05-2-11 & 292 & 26528 & 1.6 & 3.76021 & 3.6 \\
\hline 02 & CR05-2-40 & 82 & 17182 & 1.4 & 4.02755 & 3.3 \\
\hline 02 & CR05-2-35 & 312 & 88632 & 1.9 & 4.31078 & 2.3 \\
\hline 02 & CR-CR052-5 & 148 & 9839 & 1.3 & 3.63942 & 4.1 \\
\hline 02 & CR05-2-34 & 211 & 58536 & 1.7 & 4.27538 & 2.5 \\
\hline 02 & CR05-2-41 & 202 & 39717 & 1.0 & 4.46873 & 3.7 \\
\hline 02 & CR05-2-10 & 391 & 60236 & 1.5 & 4.47570 & 2.5 \\
\hline 02 & CR05-2-30 & 188 & 49003 & 2.5 & 4.52713 & 1.9 \\
\hline 02 & CR-CR052-6 & 114 & 24949 & 1.0 & 4.46861 & 1.8 \\
\hline 02 & CR-CR052-42 & 663 & 69439 & 2.3 & 4.47063 & 4.4 \\
\hline 02 & CR-CR052-48 & 233 & 44322 & 0.9 & 4.44104 & 3.2 \\
\hline 02 & CR-CR052-9 & 400 & 68877 & 2.0 & 4.11105 & 2.8 \\
\hline 02 & CR05-2-44 & 211 & 49750 & 0.8 & 4.61439 & 2.9 \\
\hline 02 & CR05-2-38 & 341 & 70382 & 1.3 & 4.69555 & 5.2 \\
\hline 02 & CR05-2-26 & 643 & 52063 & 1.5 & 4.73454 & 3.5 \\
\hline 02 & CR-CR052-10 & 858 & 33548 & 1.0 & 4.39507 & 2.0 \\
\hline 02 & CR05-2-25 & 389 & 42176 & 2.8 & 4.61640 & 2.1 \\
\hline 02 & CR05-2-32 & 104 & 6238 & 0.8 & 4.65741 & 5.1 \\
\hline 02 & CR05-2-16 & 171 & 43646 & 0.9 & 5.26278 & 2.2 \\
\hline 02 & CR-CR052-18 & 96 & 22792 & 2.1 & 5.19394 & 2.0 \\
\hline 02 & CR-CR052-17 & 149 & 2453 & 1.5 & 4.74795 & 3.4 \\
\hline 02 & CR-CR052-26 & 269 & 27610 & 2.9 & 5.01747 & 8.2 \\
\hline 02 & CR-CR052-52 & 816 & 415 & 0.1 & 0.32638 & 10.0 \\
\hline 02 & CR05-2-47 & 30 & 9832 & 0.8 & 6.69272 & 3.9 \\
\hline 02 & CR-CR052-50 & 117 & 29638 & 2.6 & 7.00559 & 1.4 \\
\hline 02 & CR05-2-1 & 514 & 19282 & 1.2 & 8.04488 & 3.2 \\
\hline 02 & CR-CR052-3 & 17 & 5623 & 1.0 & 10.98940 & 8.6 \\
\hline 02 & CR-CR052-22 & 109 & 45401 & 1.6 & 13.40416 & 1.4 \\
\hline 02 & CR-CR052-47 & 44 & 17162 & 1.5 & 14.46381 & 1.6 \\
\hline 02 & CR-CR052-59 & 117 & 63915 & 2.1 & 37.16922 & 1.2 \\
\hline 03 & $1-23-06-1-5$ & 267 & 623 & 1.9 & 0.00811 & 63.7 \\
\hline 03 & $1-23-06-1-22$ & 477 & 735 & 1.3 & 0.02341 & 14.5 \\
\hline 03 & $1-23-06-1-7$ & 270 & 935 & 1.7 & 0.03159 & 12.9 \\
\hline 03 & $1-23-06-1-11$ & 451 & 1639 & 0.6 & 0.03444 & 16.7 \\
\hline 03 & $1-23-06-1-24$ & 151 & 537 & 1.5 & 0.04051 & 18.3 \\
\hline 03 & $1-23-06-1-65$ & 690 & 4882 & 1.8 & 0.05308 & 7.5 \\
\hline 03 & $1-23-06-1-8$ & 199 & 2022 & 2.5 & 0.08070 & 8.7 \\
\hline 03 & $1-23-06-1-55$ & 280 & 4243 & 0.5 & 0.10127 & 9.1 \\
\hline 03 & $1-23-06-1-38$ & 568 & 2516 & 4.9 & 0.09753 & 5.1 \\
\hline 03 & $1-23-06-1-75$ & 250 & 2419 & 2.6 & 0.12472 & 11.1 \\
\hline 03 & $1-23-06-1-51$ & 239 & 3788 & 0.6 & 0.15985 & 7.2 \\
\hline 03 & $1-23-06-1-39$ & 450 & 7176 & 1.3 & 0.17657 & 4.1 \\
\hline 03 & $1-23-06-1-21$ & 475 & 9630 & 1.2 & 0.18000 & 3.6 \\
\hline 03 & $1-23-06-1-48$ & 185 & 4151 & 0.9 & 0.19525 & 4.9 \\
\hline 03 & $1-23-06-1-44$ & 350 & 5136 & 1.8 & 0.19113 & 7.2 \\
\hline 03 & $1-23-06-1-43$ & 118 & 3483 & 2.4 & 0.23026 & 13.2 \\
\hline 03 & $1-23-06-1-44$ & 104 & 3468 & 2.0 & 0.26127 & 10.1 \\
\hline 03 & $1-23-06-1-31$ & 273 & 5403 & 1.8 & 0.34299 & 7.0 \\
\hline 03 & $1-23-06-1-22$ & 86 & 1486 & 178.6 & 0.56233 & 7.8 \\
\hline 03 & $1-23-06-1-35$ & 265 & 7802 & 1.7 & 0.40581 & 3.0 \\
\hline 03 & $1-23-06-1-12$ & 942 & 16961 & 6.0 & 0.41054 & 1.7 \\
\hline 03 & $1-23-06-1-40$ & 381 & 4236 & 2.1 & 0.40812 & 3.9 \\
\hline 03 & $1-23-06-1-28$ & 515 & 13221 & 2.3 & 0.48208 & 4.4 \\
\hline 03 & $1-23-06-1-4$ & 365 & 7173 & 1.7 & 0.52788 & 5.1 \\
\hline 03 & $1-23-06-1-30$ & 344 & 3886 & 0.5 & 0.56432 & 5.2 \\
\hline 03 & $1-23-06-1-31$ & 174 & 4704 & 2.7 & 0.50753 & 3.3 \\
\hline 03 & $1-23-06-1-14$ & 259 & 11329 & 2.0 & 0.52292 & 3.0 \\
\hline 03 & $1-23-06-1-6$ & 235 & 8031 & 12.8 & 0.58126 & 5.8 \\
\hline 03 & $1-23-06-1-24$ & 164 & 2953 & 1.0 & 0.72313 & 8.3 \\
\hline 03 & $1-23-06-1-23$ & 135 & 7171 & 4.6 & 0.64026 & 3.6 \\
\hline 03 & $1-23-06-1-32$ & 245 & 5094 & 1.8 & 0.69224 & 3.8 \\
\hline 03 & $1-23-06-1-29$ & 446 & 3262 & 2.5 & 1.09893 & 8.0 \\
\hline 03 & $1-23-06-1-70$ & 208 & 11056 & 2.0 & 0.77940 & 2.9 \\
\hline 03 & 1-23-06-1-11 & 154 & 7674 & 2.5 & 0.79293 & 2.3 \\
\hline 03 & $1-23-06-1-3$ & 299 & 14278 & 1.7 & 0.79985 & 1.9 \\
\hline 03 & $1-23-06-1-41$ & 147 & 6171 & 3.9 & 0.78096 & 3.4 \\
\hline 03 & $1-23-06-1-19$ & 259 & 23865 & 1.1 & 0.82172 & 2.3 \\
\hline 03 & $1-23-06-1-9$ & 110 & 13807 & 2.7 & 1.45184 & 3.2 \\
\hline 03 & $1-23-06-1-29$ & 282 & 18303 & 3.6 & 1.53032 & 3.6 \\
\hline 03 & $1-23-06-1-30$ & 287 & 28343 & 6.2 & 1.64859 & 3.8 \\
\hline 03 & $1-23-06-1-42$ & 196 & 25727 & 2.6 & 1.74732 & 2.7 \\
\hline 03 & $1-23-06-1-20$ & 96 & 11615 & 5.4 & 1.77011 & 3.0 \\
\hline 03 & $1-23-06-1-17$ & 209 & 25793 & 2.4 & 1.73856 & 2.3 \\
\hline 03 & $1-23-06-1-39$ & 49 & 5445 & 3.6 & 1.89000 & 3.2 \\
\hline 03 & $1-23-06-1-74$ & 41 & 6158 & 1.2 & 1.86619 & 4.7 \\
\hline 03 & $1-23-06-1-64$ & 98 & 12390 & 2.4 & 1.75140 & 2.1 \\
\hline 03 & $1-23-06-1-54$ & 367 & 19232 & 3.4 & 1.78982 & 3.4 \\
\hline 03 & $1-23-06-1-7$ & 196 & 24051 & 1.5 & 1.87812 & 1.8 \\
\hline 03 & $1-23-06-1-33$ & 172 & 14049 & 4.5 & 2.04980 & 2.8 \\
\hline 03 & 1-23-06-1-15 & 183 & 17971 & 3.6 & 2.07774 & 2.9 \\
\hline 03 & 1-23-06-1-1 & 206 & 27471 & 5.0 & 2.16415 & 3.1 \\
\hline 03 & $1-23-06-1-63$ & 249 & 28228 & 3.0 & 2.03703 & 1.7 \\
\hline 03 & $1-23-06-1-34$ & 61 & 12151 & 1.6 & 2.26758 & 2.8 \\
\hline 03 & $1-23-06-1-33$ & 346 & 23943 & 2.7 & 1.93799 & 2.8 \\
\hline 03 & $1-23-06-1-43$ & 182 & 24027 & 3.9 & 2.56128 & 4.9 \\
\hline 03 & $1-23-06-1-40$ & 94 & 15409 & 2.9 & 2.41358 & 2.1 \\
\hline 03 & $1-23-06-1-18$ & 250 & 21809 & 3.9 & 2.66795 & 2.4 \\
\hline 03 & $1-23-06-1-2$ & 68 & 3601 & 2.1 & 2.74137 & 6.1 \\
\hline 03 & $1-23-06-1-12$ & 1129 & 90318 & 1.5 & 2.65505 & 6.5 \\
\hline 03 & $1-23-06-1-27$ & 252 & 36700 & 1.1 & 2.86131 & 3.9 \\
\hline
\end{tabular}


Kimbrough, D.L., Grove, M., Gehrels, G.E., Dorsey, R.J., Howard, K.A., Lovera, O., Aslan, A., House, P.K., and Pearthree, P.A., 2015, Detrital zircon U-Pb provenance of the Colorado River: A 5 m.y. record of incision into cover strata overlying the Colorado Plateau and adjacent regions: Geosphere, v. 11, doi:10.1130/GES00982.1.

TABLE DR7. U-Pb ZIRCON LA-ICP-MS GEOCHRONOLOGIC ANALYSES OF COLORADO RIVER BASIN \& ASSOCIATED SAMPLES.

\begin{tabular}{|c|c|c|c|c|c|c|}
\hline & & & & & & topic rat \\
\hline ID\# & Analysis & $\begin{array}{c}\text { U } \\
(\mathrm{ppm})\end{array}$ & $\begin{array}{l}206 \mathrm{~Pb} \\
204 \mathrm{~Pb}\end{array}$ & $\mathrm{U} / \mathrm{Th}$ & $\begin{array}{c}207 \mathrm{~Pb}^{*} \\
235 \mathrm{U}\end{array}$ & $\begin{array}{c} \pm \\
(\%) \\
\end{array}$ \\
\hline 03 & 1-23-06-1-72 & 158 & 33649 & 2.3 & 3.06289 & 1.4 \\
\hline 03 & $1-23-06-1-46$ & 490 & 66703 & 2.9 & 3.16521 & 4.7 \\
\hline 03 & $1-23-06-1-3$ & 268 & 32206 & 2.6 & 2.87672 & 1.8 \\
\hline 03 & $1-23-06-1-60$ & 94 & 17102 & 1.7 & 2.89627 & 1.9 \\
\hline 03 & $1-23-06-1-47$ & 123 & 24617 & 0.9 & 3.03311 & 2.4 \\
\hline 03 & $1-23-06-1-46$ & 146 & 27989 & 1.7 & 3.17128 & 1.7 \\
\hline 03 & $1-23-06-1-66$ & 178 & 39062 & 1.9 & 3.14604 & 2.0 \\
\hline 03 & $1-23-06-1-50$ & 181 & 42941 & 3.3 & 3.24411 & 1.7 \\
\hline 03 & $1-23-06-1-19$ & 276 & 41404 & 5.1 & 3.19268 & 4.3 \\
\hline 03 & $1-23-06-1-59$ & 86 & 22156 & 2.3 & 3.02718 & 1.9 \\
\hline 03 & $1-23-06-1-10$ & 176 & 29501 & 1.0 & 2.93088 & 1.9 \\
\hline 03 & $1-23-06-1-1$ & 113 & 20243 & 2.0 & 3.06729 & 1.5 \\
\hline 03 & $1-23-06-1-68$ & 180 & 41603 & 2.0 & 3.12935 & 1.6 \\
\hline 03 & $1-23-06-1-36$ & 65 & 12876 & 4.5 & 3.13213 & 2.7 \\
\hline 03 & $1-23-06-1-9$ & 203 & 14640 & 3.6 & 3.12224 & 4.9 \\
\hline 03 & $1-23-06-1-5$ & 337 & 43368 & 3.2 & 3.41775 & 2.5 \\
\hline 03 & $1-23-06-1-50$ & 95 & 14696 & 2.0 & 3.35773 & 5.9 \\
\hline 03 & $1-23-06-1-71$ & 90 & 17221 & 1.9 & 4.05981 & 1.9 \\
\hline 03 & $1-23-06-1-25$ & 121 & 22901 & 3.3 & 3.96100 & 2.9 \\
\hline 03 & $1-23-06-1-27$ & 1042 & 43526 & 2.0 & 4.07198 & 3.4 \\
\hline 03 & $1-23-06-1-67$ & 92 & 22422 & 4.7 & 4.06163 & 3.6 \\
\hline 03 & $1-23-06-1-15$ & 133 & 25633 & 0.8 & 3.67522 & 3.3 \\
\hline 03 & $1-23-06-1-61$ & 584 & 115425 & 21.4 & 3.94951 & 2.3 \\
\hline 03 & $1-23-06-1-16$ & 463 & 27355 & 1.6 & 3.77519 & 4.6 \\
\hline 03 & $1-23-06-1-45$ & 90 & 23473 & 1.0 & 3.94268 & 2.9 \\
\hline 03 & $1-23-06-1-17$ & 100 & 24948 & 3.4 & 4.23639 & 4.1 \\
\hline 03 & $1-23-06-1-13$ & 266 & 28313 & 2.9 & 4.16053 & 2.4 \\
\hline 03 & $1-23-06-1-62$ & 211 & 56363 & 1.6 & 3.95255 & 1.4 \\
\hline 03 & $1-23-06-1-49$ & 311 & 5536 & 2.0 & 3.56392 & 2.8 \\
\hline 03 & $1-23-06-1-37$ & 76 & 18470 & 1.0 & 4.14622 & 1.8 \\
\hline 03 & $1-23-06-1-58$ & 265 & 63462 & 4.8 & 4.39246 & 5.7 \\
\hline 03 & $1-23-06-1-47$ & 160 & 38119 & 3.7 & 4.46581 & 5.7 \\
\hline 03 & $1-23-06-1-53$ & 76 & 3551 & 1.2 & 4.08523 & 6.7 \\
\hline 03 & $1-23-06-1-18$ & 347 & 90900 & 3.5 & 4.25847 & 2.6 \\
\hline 03 & $1-23-06-1-6$ & 348 & 67582 & 1.3 & 4.02693 & 2.6 \\
\hline 03 & $1-23-06-1-32$ & 212 & 27902 & 1.4 & 4.22957 & 4.9 \\
\hline 03 & $1-23-06-1-21$ & 225 & 28413 & 2.0 & 4.44164 & 3.2 \\
\hline 03 & $1-23-06-1-37$ & 264 & 30185 & 5.7 & 4.39455 & 2.4 \\
\hline 03 & $1-23-06-1-48$ & 244 & 66272 & 7.7 & 4.45247 & 3.5 \\
\hline 03 & $1-23-06-1-41$ & 270 & 95208 & 3.4 & 4.47743 & 2.0 \\
\hline 03 & $1-23-06-1-13$ & 100 & 28668 & 3.1 & 4.59684 & 1.4 \\
\hline 03 & $1-23-06-1-34$ & 338 & 41992 & 5.9 & 4.58103 & 2.9 \\
\hline 03 & $1-23-06-1-2$ & 177 & 45797 & 2.7 & 4.55403 & 1.9 \\
\hline 03 & $1-23-06-1-69$ & 446 & 29875 & 3.5 & 4.53932 & 6.5 \\
\hline 03 & $1-23-06-1-20$ & 200 & 20644 & 1.7 & 3.99574 & 3.2 \\
\hline 03 & $1-23-06-1-38$ & 747 & 114790 & 1.7 & 4.34743 & 1.4 \\
\hline 03 & $1-23-06-1-36$ & 362 & 61561 & 2.2 & 5.22134 & 2.0 \\
\hline 03 & $1-23-06-1-4$ & 394 & 21580 & 7.1 & 5.75293 & 3.8 \\
\hline 03 & $1-23-06-1-10$ & 223 & 60005 & 4.2 & 6.01319 & 2.5 \\
\hline 03 & $1-23-06-1-45$ & 188 & 18152 & 8.6 & 6.24319 & 5.2 \\
\hline 03 & $1-23-06-1-26$ & 381 & 95573 & 8.6 & 6.28396 & 1.7 \\
\hline 03 & $1-23-06-1-73$ & 119 & 35272 & 1.3 & 6.45778 & 2.3 \\
\hline 03 & $1-23-06-1-56$ & 100 & 22771 & 1.2 & 8.29378 & 3.8 \\
\hline 03 & $1-23-06-1-14$ & 42 & 21147 & 2.9 & 12.66141 & 1.7 \\
\hline 03 & $1-23-06-1-28$ & 41 & 9840 & 0.5 & 11.93496 & 2.1 \\
\hline 04 & YUMA-21 & 554 & 882 & 1.5 & 0.0056 & 39.7 \\
\hline 04 & YUMA-12 & 272 & 1077 & 0.5 & 0.0107 & 70.7 \\
\hline 04 & YUMA-41 & 216 & 552 & 0.9 & 0.0066 & 53.0 \\
\hline 04 & YUMA-32 & 78 & 510 & 0.6 & 0.0083 & 330.1 \\
\hline 04 & YUMA-65 & 297 & 1296 & 1.0 & 0.0180 & 20.9 \\
\hline 04 & YUMA-2 & 43 & 471 & 0.7 & 0.0335 & 65.5 \\
\hline 04 & YUMA-50 & 301 & 939 & 1.8 & 0.0704 & 11.7 \\
\hline 04 & YUMA-8 & 1376 & 906 & 0.7 & 0.1252 & 15.9 \\
\hline 04 & YUMA-43 & 140 & 1632 & 1.6 & 0.0518 & 20.9 \\
\hline 04 & YUMA-36 & 354 & 3594 & 1.7 & 0.0703 & 6.5 \\
\hline 04 & YUMA-4 & 106 & 4851 & 2.7 & 0.0583 & 20.1 \\
\hline 04 & YUMA-18 & 129 & 1725 & 0.3 & 0.1496 & 18.5 \\
\hline 04 & YUMA-69 & 978 & 7599 & 0.3 & 0.2066 & 16.5 \\
\hline 04 & YUMA-101 & 513 & 16341 & 1.1 & 0.1710 & 4.0 \\
\hline 04 & YUMA-3 & 68 & 4023 & 0.7 & 0.2246 & 7.8 \\
\hline 04 & YUMA-6 & 29 & 2373 & 1.3 & 0.2362 & 17.6 \\
\hline 04 & YUMA-63 & 460 & 11295 & 0.9 & 0.4129 & 10.7 \\
\hline 04 & YUMA-88 & 560 & 11424 & 0.5 & 0.4565 & 11.3 \\
\hline 04 & YUMA-37 & 440 & 20412 & 1.5 & 0.4524 & 3.6 \\
\hline 04 & YUMA-26 & 153 & 10782 & 1.2 & 0.7509 & 8.4 \\
\hline 04 & YUMA-10 & 189 & 15864 & 1.0 & 0.4789 & 3.2 \\
\hline 04 & YUMA-77 & 559 & 8973 & 1.6 & 0.5685 & 6.6 \\
\hline 04 & YUMA-68 & 349 & 5412 & 1.1 & 0.5902 & 2.5 \\
\hline 04 & YUMA-45 & 285 & 14646 & 2.8 & 0.6058 & 3.1 \\
\hline 04 & YUMA-78 & 108 & 5115 & 1.2 & 0.7895 & 9.4 \\
\hline 04 & YUMA-86 & 137 & 17982 & 5.1 & 0.7004 & 2.9 \\
\hline 04 & YUMA-28 & 748 & 125778 & 6.6 & 1.5008 & 2.9 \\
\hline 04 & YUMA-33 & 154 & 29013 & 1.7 & 1.7476 & 2.0 \\
\hline 04 & YUMA-92 & 182 & 31170 & 2.1 & 1.7725 & 2.5 \\
\hline 04 & YUMA-20 & 186 & 31428 & 1.0 & 1.8607 & 3.1 \\
\hline 04 & YUMA-25 & 37 & 9015 & 0.8 & 1.8445 & 5.1 \\
\hline 04 & YUMA-17 & 241 & 40356 & 3.1 & 1.8203 & 1.8 \\
\hline 04 & YUMA-66 & 135 & 20412 & 0.8 & 1.8757 & 1.4 \\
\hline 04 & YUMA-51 & 11 & 3708 & 2.4 & 1.9873 & 5.4 \\
\hline 04 & YUMA-96 & 59 & 14682 & 1.0 & 1.9557 & 1.4 \\
\hline
\end{tabular}


Kimbrough, D.L., Grove, M., Gehrels, G.E., Dorsey, R.J., Howard, K.A., Lovera, O., Aslan, A., House, P.K., and Pearthree, P.A., 2015, Detrital zircon U-Pb provenance of the Colorado River: A 5 m.y. record of incision into cover strata overlying the Colorado Plateau and adjacent regions: Geosphere, v. 11, doi:10.1130/GES00982.1.

TABLE DR7. U-Pb ZIRCON LA-ICP-MS GEOCHRONOLOGIC ANALYSES OF COLORADO RIVER BASIN \& ASSOCIATED SAMPLES.

\begin{tabular}{|c|c|c|c|c|c|c|}
\hline & & & & & & opic ra \\
\hline ID\# & Analysis & $\begin{array}{c}\text { U } \\
(\mathrm{ppm})\end{array}$ & $\begin{array}{l}206 \mathrm{~Pb} \\
204 \mathrm{~Pb} \\
\end{array}$ & U/Th & $\begin{array}{c}207 \mathrm{~Pb}^{*} \\
235 \mathrm{U}\end{array}$ & $\begin{array}{c} \pm \\
(\%)\end{array}$ \\
\hline 04 & YUMA-16 & 92 & 18450 & 2.0 & 1.8392 & 3.3 \\
\hline 04 & YUMA-34 & 245 & 82293 & 8.8 & 1.9100 & 2.9 \\
\hline 04 & YUMA-23 & 86 & 19986 & 1.6 & 1.9629 & 2.2 \\
\hline 04 & YUMA-93 & 49 & 9594 & 1.5 & 2.0547 & 2.4 \\
\hline 04 & YUMA-79 & 204 & 41010 & 1.8 & 2.2735 & 1.6 \\
\hline 04 & YUMA-13 & 85 & 26229 & 1.6 & 2.3845 & 3.1 \\
\hline 04 & YUMA-55 & 87 & 20967 & 1.0 & 2.2229 & 4.2 \\
\hline 04 & YUMA-71 & 107 & 15882 & 1.0 & 2.2846 & 4.8 \\
\hline 04 & YUMA-46 & 260 & 68397 & 1.1 & 2.8833 & 1.8 \\
\hline 04 & YUMA-97 & 321 & 100053 & 0.9 & 3.0180 & 2.4 \\
\hline 04 & YUMA-15 & 73 & 33834 & 1.2 & 2.9863 & 2.9 \\
\hline 04 & YUMA-61 & 443 & 91263 & 2.5 & 2.8252 & 1.7 \\
\hline 04 & YUMA-103 & 268 & 53193 & 3.4 & 2.9863 & 2.7 \\
\hline 04 & YUMA-19 & 359 & 45921 & 1.9 & 2.8595 & 2.9 \\
\hline 04 & YUMA-104 & 279 & 71298 & 0.8 & 2.9607 & 2.4 \\
\hline 04 & YUMA-1 & 105 & 41175 & 1.2 & 3.1296 & 3.6 \\
\hline 04 & YUMA-99 & 148 & 29451 & 1.6 & 2.8933 & 2.9 \\
\hline 04 & YUMA-11 & 49 & 11553 & 1.4 & 3.0363 & 2.7 \\
\hline 04 & YUMA-89 & 863 & 43332 & 4.2 & 2.7228 & 1.5 \\
\hline 04 & YUMA-81 & 281 & 61041 & 1.1 & 3.1336 & 2.0 \\
\hline 04 & YUMA-47 & 147 & 12345 & 0.8 & 2.8068 & 2.3 \\
\hline 04 & YUMA-70 & 343 & 46671 & 2.4 & 2.9097 & 3.2 \\
\hline 04 & YUMA-80 & 60 & 20016 & 1.3 & 3.4333 & 2.3 \\
\hline 04 & YUMA-73 & 117 & 4311 & 0.9 & 2.6842 & 3.6 \\
\hline 04 & YUMA-98 & 175 & 18834 & 1.4 & 3.3198 & 4.9 \\
\hline 04 & YUMA-53 & 324 & 109182 & 1.9 & 4.0015 & 3.0 \\
\hline 04 & YUMA-90 & 153 & 47961 & 1.2 & 4.0389 & 4.6 \\
\hline 04 & YUMA-100 & 87 & 27990 & 2.4 & 3.9830 & 1.8 \\
\hline 04 & YUMA-59 & 81 & 38532 & 2.6 & 3.9685 & 3.3 \\
\hline 04 & YUMA-44 & 98 & 19371 & 1.0 & 3.9213 & 1.7 \\
\hline 04 & YUMA-57 & 47 & 33594 & 0.9 & 4.1372 & 3.6 \\
\hline 04 & YUMA-54 & 53 & 18225 & 1.4 & 3.9927 & 2.4 \\
\hline 04 & YUMA-29 & 156 & 48435 & 1.5 & 4.0676 & 3.8 \\
\hline 04 & YUMA-24 & 120 & 40278 & 0.7 & 4.1534 & 1.5 \\
\hline 04 & YUMA-84 & 123 & 19662 & 1.3 & 3.5621 & 2.9 \\
\hline 04 & YUMA-82 & 102 & 26199 & 0.8 & 4.0314 & 1.8 \\
\hline 04 & YUMA-85 & 70 & 27771 & 1.3 & 4.1325 & 2.7 \\
\hline 04 & YUMA-83 & 130 & 45612 & 1.0 & 4.0377 & 2.3 \\
\hline 04 & YUMA-91 & 230 & 107211 & 1.2 & 4.2713 & 1.4 \\
\hline 04 & YUMA-75 & 619 & 191331 & 2.2 & 4.0236 & 2.7 \\
\hline 04 & YUMA-27 & 230 & 65757 & 0.6 & 4.1737 & 1.6 \\
\hline 04 & YUMA-35 & 139 & 56259 & 1.5 & 4.3718 & 2.4 \\
\hline 04 & YUMA-40 & 557 & 243894 & 2.8 & 4.3017 & 2.1 \\
\hline 04 & YUMA-62 & 168 & 40530 & 1.0 & 4.1723 & 3.9 \\
\hline 04 & YUMA-22 & 118 & 23307 & 1.1 & 4.2317 & 3.6 \\
\hline 04 & YUMA-7 & 82 & 41652 & 0.7 & 4.2284 & 4.7 \\
\hline 04 & YUMA-31 & 120 & 8601 & 1.2 & 3.8555 & 4.3 \\
\hline 04 & YUMA-5 & 275 & 38820 & 1.0 & 3.7615 & 4.5 \\
\hline 04 & YUMA-67 & 2021 & 231417 & 5.7 & 4.2101 & 2.0 \\
\hline 04 & YUMA-74 & 421 & 120666 & 2.5 & 4.2382 & 2.5 \\
\hline 04 & YUMA-39 & 205 & 69951 & 1.7 & 4.3705 & 1.7 \\
\hline 04 & YUMA-48 & 375 & 111462 & 1.4 & 3.5525 & 4.3 \\
\hline 04 & YUMA-56 & 426 & 80520 & 2.3 & 4.4268 & 2.9 \\
\hline 04 & YUMA-72 & 198 & 20367 & 1.8 & 4.0506 & 5.1 \\
\hline 04 & YUMA-102 & 296 & 28800 & 1.2 & 4.6162 & 3.0 \\
\hline 04 & YUMA-9 & 1041 & 69012 & 4.1 & 4.0401 & 7.8 \\
\hline 04 & YUMA-58 & 74 & 21309 & 1.8 & 4.4484 & 3.2 \\
\hline 04 & YUMA-76 & 95 & 30879 & 0.8 & 7.2446 & 4.2 \\
\hline 04 & YUMA-64 & 16 & 7647 & 0.1 & 12.0431 & 4.2 \\
\hline 05 & FELIPITO-30 & 2967 & 3618 & 0.5 & 0.0201 & 3.0 \\
\hline 05 & FELIPITO-33 & 76 & 1322 & 1.2 & 0.0231 & 75.1 \\
\hline 05 & FELIPITO-93 & 491 & 3956 & 1.3 & 0.0712 & 6.9 \\
\hline 05 & FELIPITO-89 & 241 & 1422 & 0.3 & 0.0631 & 10.1 \\
\hline 05 & FELIPITO-65 & 538 & 3414 & 1.4 & 0.1113 & 3.9 \\
\hline 05 & FELIPITO-97 & 289 & 2580 & 1.1 & 0.0997 & 7.2 \\
\hline 05 & FELIPITO-35 & 145 & 4542 & 0.7 & 0.1449 & 11.4 \\
\hline 05 & FELIPITO-12 & 261 & 5926 & 1.3 & 0.2662 & 2.4 \\
\hline 05 & FELIPITO-18 & 202 & 2148 & 0.9 & 0.4052 & 36.2 \\
\hline 05 & FELIPITO-28 & 619 & 22364 & 1.2 & 0.4794 & 1.9 \\
\hline 05 & FELIPITO-5 & 447 & 8126 & 1.7 & 0.5571 & 20.2 \\
\hline 05 & FELIPITO-78 & 311 & 24200 & 1.3 & 0.4985 & 1.8 \\
\hline 05 & FELIPITO-9 & 691 & 1762 & 4.9 & 0.6476 & 7.8 \\
\hline 05 & FELIPITO-56 & 303 & 19686 & 1.5 & 0.5020 & 2.3 \\
\hline 05 & FELIPITO-83 & 99 & 7500 & 1.7 & 0.5143 & 3.3 \\
\hline 05 & FELIPITO-72 & 317 & 8418 & 0.6 & 0.5365 & 4.6 \\
\hline 05 & FELIPITO-62 & 592 & 26400 & 1.0 & 0.5467 & 2.0 \\
\hline 05 & FELIPITO-91 & 169 & 8596 & 1.1 & 0.5421 & 2.4 \\
\hline 05 & FELIPITO-77 & 456 & 17010 & 10.1 & 0.5640 & 2.0 \\
\hline 05 & FELIPITO-67 & 106 & 9428 & 2.7 & 0.6098 & 3.5 \\
\hline 05 & FELIPITO-41 & 176 & 4524 & 1.4 & 0.9170 & 31.0 \\
\hline 05 & FELIPITO-70 & 87 & 2934 & 1.1 & 0.7145 & 4.2 \\
\hline 05 & FELIPITO-13 & 441 & 2152 & 2.9 & 0.8959 & 5.5 \\
\hline 05 & FELIPITO-24 & 603 & 49742 & 4.2 & 0.7360 & 2.5 \\
\hline 05 & FELIPITO-80 & 151 & 7476 & 1.4 & 0.7215 & 2.8 \\
\hline 05 & FELIPITO-68 & 189 & 5958 & 2.7 & 0.7641 & 3.0 \\
\hline 05 & FELIPITO-25 & 535 & 54464 & 4.4 & 0.7515 & 2.1 \\
\hline 05 & FELIPITO-54 & 76 & 3848 & 1.9 & 0.7243 & 3.3 \\
\hline 05 & FELIPITO-88 & 110 & 6988 & 1.4 & 0.7241 & 2.5 \\
\hline 05 & FELIPITO-76 & 253 & 15686 & 1.5 & 0.8011 & 1.7 \\
\hline 05 & FELIPITO-44 & 203 & 12002 & 1.2 & 0.8554 & 1.6 \\
\hline
\end{tabular}


Kimbrough, D.L., Grove, M., Gehrels, G.E., Dorsey, R.J., Howard, K.A., Lovera, O., Aslan, A., House, P.K., and Pearthree, P.A., 2015, Detrital zircon U-Pb provenance of the Colorado River: A 5 m.y. record of incision into cover strata overlying the Colorado Plateau and adjacent regions: Geosphere, v. 11, doi:10.1130/GES00982.1.

TABLE DR7. U-Pb ZIRCON LA-ICP-MS GEOCHRONOLOGIC ANALYSES OF COLORADO RIVER BASIN \& ASSOCIATED SAMPLES.

\begin{tabular}{|c|c|c|c|c|c|c|}
\hline & & & & & & topic rat \\
\hline ID\# & Analysis & $\begin{array}{c}\text { U } \\
(\mathrm{ppm})\end{array}$ & $\begin{array}{l}206 \mathrm{~Pb} \\
204 \mathrm{~Pb}\end{array}$ & $\mathrm{U} / \mathrm{Th}$ & $\begin{array}{c}207 \mathrm{~Pb}^{*} \\
235 \mathrm{U}\end{array}$ & $\begin{array}{c} \pm \\
(\%) \\
\end{array}$ \\
\hline 05 & $\begin{array}{l}\text { FELIPITO-42 } \\
\end{array}$ & 106 & 5640 & 1.7 & 0.9178 & 3.7 \\
\hline 05 & FELIPITO-55 & 259 & 9190 & 1.4 & 0.8943 & 1.8 \\
\hline 05 & FELIPITO-7 & 153 & 8236 & 2.1 & 0.8928 & 3.0 \\
\hline 05 & FELIPITO-100 & 209 & 8370 & 1.4 & 0.9049 & 2.8 \\
\hline 05 & FELIPITO-34 & 318 & 4538 & 20.7 & 1.0335 & 4.4 \\
\hline 05 & FELIPITO-74 & 309 & 14742 & 0.8 & 1.1682 & 3.8 \\
\hline 05 & FELIPITO-2 & 292 & 28554 & 1.2 & 1.5268 & 2.0 \\
\hline 05 & FELIPITO-16 & 106 & 9528 & 2.0 & 1.6210 & 2.0 \\
\hline 05 & FELIPITO-73 & 374 & 19202 & 1.4 & 1.5902 & 2.2 \\
\hline 05 & FELIPITO-59 & 271 & 28764 & 1.6 & 1.7576 & 2.1 \\
\hline 05 & FELIPITO-81 & 227 & 39836 & 2.6 & 1.6991 & 3.1 \\
\hline 05 & FELIPITO-101 & 215 & 23716 & 1.8 & 1.7507 & 2.3 \\
\hline 05 & FELIPITO-51 & 239 & 19442 & 1.4 & 1.7443 & 2.9 \\
\hline 05 & FELIPITO-96 & 79 & 6360 & 1.9 & 1.8479 & 2.6 \\
\hline 05 & FELIPITO-48 & 138 & 23792 & 1.7 & 1.8046 & 2.8 \\
\hline 05 & FELIPITO-58 & 391 & 37976 & 1.6 & 1.8967 & 2.1 \\
\hline 05 & FELIPITO-20 & 134 & 7848 & 1.7 & 1.9213 & 2.5 \\
\hline 05 & FELIPITO-43 & 207 & 20520 & 1.1 & 2.0350 & 4.2 \\
\hline 05 & FELIPITO-79 & 86 & 11542 & 0.9 & 2.2414 & 1.7 \\
\hline 05 & FELIPITO-36 & 170 & 18338 & 2.3 & 2.4109 & 2.6 \\
\hline 05 & FELIPITO-14 & 119 & 11160 & 1.5 & 2.2603 & 2.3 \\
\hline 05 & FELIPITO-19 & 461 & 30766 & 1.8 & 2.8687 & 2.0 \\
\hline 05 & FELIPITO-45 & 247 & 35908 & 1.7 & 3.0282 & 1.7 \\
\hline 05 & FELIPITO-82 & 83 & 19820 & 1.7 & 3.1634 & 2.1 \\
\hline 05 & FELIPITO-95 & 164 & 21428 & 1.3 & 3.2753 & 1.9 \\
\hline 05 & FELIPITO-26 & 180 & 25744 & 1.2 & 3.0491 & 2.6 \\
\hline 05 & FELIPITO-64 & 193 & 38334 & 1.7 & 3.2238 & 2.1 \\
\hline 05 & FELIPITO-94 & 105 & 14878 & 0.8 & 3.4574 & 1.7 \\
\hline 05 & FELIPITO-29 & 132 & 27322 & 2.2 & 3.4066 & 2.8 \\
\hline 05 & FELIPITO-40 & 256 & 35580 & 2.2 & 3.6490 & 1.9 \\
\hline 05 & FELIPITO-52 & 82 & 14902 & 1.8 & 4.0126 & 3.7 \\
\hline 05 & FELIPITO-10 & 155 & 37278 & 1.8 & 3.7616 & 5.0 \\
\hline 05 & FELIPITO-69 & 134 & 37154 & 0.6 & 4.1141 & 1.7 \\
\hline 05 & FELIPITO-27 & 129 & 36450 & 1.1 & 3.8583 & 3.6 \\
\hline 05 & FELIPITO-103 & 126 & 16756 & 0.9 & 4.0683 & 3.9 \\
\hline 05 & FELIPITO-23 & 126 & 18882 & 1.0 & 4.0589 & 1.9 \\
\hline 05 & FELIPITO-66 & 114 & 20982 & 1.2 & 4.0373 & 2.6 \\
\hline 05 & FELIPITO-11 & 1126 & 63232 & 0.6 & 3.2114 & 1.6 \\
\hline 05 & FELIPITO-60 & 66 & 10448 & 2.2 & 4.5048 & 2.0 \\
\hline 05 & FELIPITO-46 & 122 & 34112 & 2.0 & 4.3145 & 3.1 \\
\hline 05 & FELIPITO-92 & 639 & 73916 & 1.6 & 4.4347 & 2.8 \\
\hline 05 & FELIPITO-85 & 216 & 18910 & 1.7 & 3.7906 & 2.4 \\
\hline 05 & FELIPITO-6 & 117 & 19568 & 1.3 & 4.4502 & 2.5 \\
\hline 05 & FELIPITO-86 & 258 & 5634 & 2.5 & 4.0023 & 5.3 \\
\hline 05 & FELIPITO-1 & 348 & 66160 & 4.2 & 4.4430 & 1.5 \\
\hline 05 & FELIPITO-15 & 422 & 55210 & 2.6 & 4.3659 & 1.9 \\
\hline 05 & FELIPITO-17 & 256 & 40680 & 1.4 & 4.2588 & 2.7 \\
\hline 05 & FELIPITO-38 & 219 & 67236 & 2.2 & 4.5313 & 4.7 \\
\hline 05 & FELIPITO-3 & 149 & 27090 & 1.5 & 4.3575 & 2.5 \\
\hline 05 & FELIPITO-63 & 206 & 41512 & 1.7 & 4.7756 & 1.8 \\
\hline 05 & FELIPITO-50 & 106 & 13710 & 2.3 & 4.6514 & 3.1 \\
\hline 05 & FELIPITO-8 & 446 & 53838 & 3.9 & 4.5626 & 2.2 \\
\hline 05 & FELIPITO-75 & 325 & 56724 & 7.1 & 4.7707 & 2.6 \\
\hline 05 & FELIPITO-31 & 274 & 33464 & 3.5 & 4.8122 & 3.4 \\
\hline 05 & FELIPITO-98 & 179 & 32568 & 0.6 & 5.1540 & 1.6 \\
\hline 05 & FELIPITO-57 & 248 & 40304 & 2.3 & 3.5391 & 1.4 \\
\hline 05 & FELIPITO-22 & 341 & 2924 & 0.6 & 4.3732 & 2.8 \\
\hline 05 & FELIPITO-53 & 333 & 31426 & 3.5 & 4.8441 & 1.9 \\
\hline 05 & FELIPITO-21 & 126 & 27824 & 1.4 & 5.8771 & 1.6 \\
\hline 05 & FELIPITO-49 & 35 & 10338 & 2.9 & 12.8825 & 2.7 \\
\hline 05 & FELIPITO-99 & 65 & 20062 & 1.2 & 14.4532 & 1.6 \\
\hline 05 & FELIPITO-71 & 107 & 23722 & 2.4 & 12.3492 & 3.2 \\
\hline 06 & SClara-56 & 247 & 2118 & 1.3 & 0.0459 & 21.3 \\
\hline 06 & SClara-12 & 60 & 1314 & 1.4 & 0.0348 & 106.9 \\
\hline 06 & SClara-90 & 77 & 1767 & 1.8 & 0.0979 & 12.1 \\
\hline 06 & SClara-44 & 124 & 804 & 1.4 & 0.1549 & 20.5 \\
\hline 06 & SClara-95 & 403 & 4929 & 1.0 & 0.0904 & 6.1 \\
\hline 06 & SClara-8 & 290 & 4008 & 1.0 & 0.1245 & 6.4 \\
\hline 06 & SClara-72 & 292 & 7668 & 1.1 & 0.1792 & 4.7 \\
\hline 06 & SClara-41 & 405 & 13575 & 1.3 & 0.1693 & 4.6 \\
\hline 06 & SClara-19 & 370 & 10020 & 1.0 & 0.1764 & 5.2 \\
\hline 06 & SClara-51 & 757 & 44280 & 10.3 & 0.1944 & 2.2 \\
\hline 06 & SClara-52 & 470 & 17373 & 1.6 & 0.1976 & 4.5 \\
\hline 06 & SClara-101 & 148 & 4647 & 0.7 & 0.2136 & 4.9 \\
\hline 06 & SClara-9 & 82 & 3696 & 0.8 & 0.3468 & 10.4 \\
\hline 06 & SClara-100 & 779 & 1245 & 0.9 & 0.5575 & 3.5 \\
\hline 06 & SClara-57 & 279 & 7977 & 0.7 & 0.3545 & 2.1 \\
\hline 06 & SClara-10 & 97 & 2373 & 0.5 & 0.3993 & 7.0 \\
\hline 06 & SClara-28 & 505 & 23682 & 1.1 & 0.4430 & 3.2 \\
\hline 06 & SClara-83 & 258 & 9576 & 1.4 & 0.4987 & 4.0 \\
\hline 06 & SClara-38 & 277 & 9096 & 1.5 & 0.4774 & 4.3 \\
\hline 06 & SClara-84 & 89 & 5514 & 1.5 & 0.4751 & 12.6 \\
\hline 06 & SClara-3 & 554 & 42399 & 0.7 & 0.4952 & 1.4 \\
\hline 06 & SClara-26 & 161 & 8739 & 1.4 & 0.5052 & 4.5 \\
\hline 06 & SClara-4 & 185 & 12120 & 1.2 & 0.5252 & 2.3 \\
\hline 06 & SClara-76 & 78 & 7539 & 1.7 & 0.6375 & 6.0 \\
\hline 06 & SClara-20 & 105 & 6456 & 1.0 & 0.6401 & 2.9 \\
\hline 06 & SClara-17 & 489 & 2778 & 0.8 & 0.9160 & 6.8 \\
\hline 06 & SClara-89 & 27 & 4062 & 0.6 & 0.7303 & 8.3 \\
\hline 06 & SClara-46 & 237 & 10974 & 2.9 & 0.8525 & 6.3 \\
\hline
\end{tabular}


Kimbrough, D.L., Grove, M., Gehrels, G.E., Dorsey, R.J., Howard, K.A., Lovera, O., Aslan, A., House, P.K., and Pearthree, P.A., 2015, Detrital zircon U-Pb provenance of the Colorado River: A 5 m.y. record of incision into cover strata overlying the Colorado Plateau and adjacent regions: Geosphere, v. 11, doi:10.1130/GES00982.1.

TABLE DR7. U-Pb ZIRCON LA-ICP-MS GEOCHRONOLOGIC ANALYSES OF COLORADO RIVER BASIN \& ASSOCIATED SAMPLES.

\begin{tabular}{|c|c|c|c|c|c|c|}
\hline & & & & & & opic ra \\
\hline ID\# & Analysis & $\begin{array}{c}\text { U } \\
(\mathrm{ppm})\end{array}$ & $\begin{array}{l}206 \mathrm{~Pb} \\
204 \mathrm{~Pb} \\
\end{array}$ & U/Th & $\begin{array}{c}207 \mathrm{~Pb}^{*} \\
235 \mathrm{U}\end{array}$ & $\begin{array}{c} \pm \\
(\%)\end{array}$ \\
\hline 06 & SClara-2 & 182 & 9096 & 1.0 & 0.8468 & 3.3 \\
\hline 06 & SClara-91 & 742 & 38712 & 4.0 & 0.8451 & 3.0 \\
\hline 06 & SClara-93 & 240 & 12462 & 0.9 & 0.8539 & 3.2 \\
\hline 06 & SClara-32 & 300 & 20559 & 1.2 & 0.8279 & 2.8 \\
\hline 06 & SClara-47 & 118 & 33123 & 1.3 & 1.6383 & 4.2 \\
\hline 06 & SClara-54 & 585 & 46836 & 1.3 & 1.7709 & 1.7 \\
\hline 06 & SClara-75 & 191 & 41310 & 3.0 & 1.8694 & 2.2 \\
\hline 06 & SClara-22 & 116 & 19920 & 1.5 & 1.8408 & 2.8 \\
\hline 06 & SClara-69 & 112 & 13929 & 1.8 & 1.8718 & 4.6 \\
\hline 06 & SClara-13 & 83 & 28134 & 3.1 & 1.9705 & 4.6 \\
\hline 06 & SClara-11 & 61 & 13275 & 0.8 & 1.9943 & 5.3 \\
\hline 06 & SClara-92 & 207 & 12567 & 0.9 & 1.8248 & 4.7 \\
\hline 06 & SClara-50 & 135 & 36702 & 2.0 & 2.1559 & 4.9 \\
\hline 06 & SClara-68 & 85 & 13437 & 1.6 & 2.1090 & 2.8 \\
\hline 06 & SClara-48 & 100 & 27432 & 1.1 & 2.2865 & 2.7 \\
\hline 06 & SClara-71 & 98 & 35913 & 2.0 & 2.1911 & 3.4 \\
\hline 06 & SClara-15 & 161 & 29799 & 2.4 & 2.1222 & 4.1 \\
\hline 06 & SClara-37 & 259 & 5013 & 1.7 & 1.8553 & 5.3 \\
\hline 06 & SClara-79 & 377 & 64305 & 1.9 & 2.6088 & 1.8 \\
\hline 06 & SClara-7 & 91 & 26316 & 1.3 & 3.0297 & 3.4 \\
\hline 06 & SClara-85 & 366 & 50775 & 0.9 & 3.0223 & 2.3 \\
\hline 06 & SClara-1 & 130 & 35028 & 1.3 & 3.0514 & 2.8 \\
\hline 06 & SClara-27 & 110 & 21513 & 0.9 & 3.0998 & 2.4 \\
\hline 06 & SClara-16 & 161 & 46938 & 1.0 & 3.1873 & 3.2 \\
\hline 06 & SClara-60 & 2997 & 81816 & 2.5 & 3.1841 & 2.3 \\
\hline 06 & SClara-30 & 149 & 8679 & 1.4 & 3.2603 & 3.1 \\
\hline 06 & SClara-86 & 898 & 347028 & 55.3 & 3.8184 & 3.0 \\
\hline 06 & SClara-29 & 151 & 14862 & 1.4 & 3.4014 & 3.8 \\
\hline 06 & SClara-94 & 313 & 73773 & 1.2 & 3.9262 & 3.2 \\
\hline 06 & SClara-25 & 314 & 98220 & 1.5 & 4.1364 & 3.6 \\
\hline 06 & SClara-35 & 137 & 10182 & 0.6 & 3.8117 & 3.9 \\
\hline 06 & SClara-59 & 383 & 46881 & 1.3 & 4.2213 & 1.7 \\
\hline 06 & SClara-70 & 365 & 117465 & 69.9 & 3.8323 & 1.5 \\
\hline 06 & SClara-53 & 145 & 38079 & 0.9 & 4.2229 & 1.6 \\
\hline 06 & SClara-55 & 207 & 67560 & 2.1 & 4.2809 & 5.8 \\
\hline 06 & SClara-33 & 585 & 104592 & 2.1 & 4.2428 & 2.2 \\
\hline 06 & SClara-64 & 314 & 52773 & 1.6 & 4.1439 & 3.5 \\
\hline 06 & SClara-14 & 342 & 113007 & 5.8 & 4.2404 & 5.1 \\
\hline 06 & SClara-34 & 2509 & 33786 & 1.7 & 4.0655 & 2.5 \\
\hline 06 & SClara-45 & 139 & 17424 & 1.5 & 3.8144 & 3.9 \\
\hline 06 & SClara-65 & 270 & 51642 & 0.8 & 4.3498 & 1.4 \\
\hline 06 & SClara-36 & 339 & 52866 & 1.1 & 4.1462 & 4.3 \\
\hline 06 & SClara-80 & 293 & 72102 & 1.6 & 4.4261 & 1.4 \\
\hline 06 & SClara-66 & 429 & 84057 & 1.4 & 4.3430 & 2.1 \\
\hline 06 & SClara-62 & 215 & 49797 & 1.1 & 4.2917 & 6.1 \\
\hline 06 & SClara-74 & 87 & 24681 & 3.1 & 4.3669 & 1.7 \\
\hline 06 & SClara-40 & 542 & 37788 & 0.5 & 4.0295 & 2.3 \\
\hline 06 & SClara-21 & 61 & 10557 & 0.9 & 4.3299 & 2.7 \\
\hline 06 & SClara-63 & 287 & 71463 & 3.7 & 4.4303 & 2.3 \\
\hline 06 & SClara-61 & 153 & 49215 & 2.0 & 4.4466 & 1.4 \\
\hline 06 & SClara-96 & 143 & 36882 & 1.0 & 4.3385 & 2.0 \\
\hline 06 & SClara-99 & 392 & 23643 & 2.7 & 4.6921 & 2.6 \\
\hline 06 & SClara-81 & 374 & 62910 & 1.6 & 4.6471 & 2.4 \\
\hline 06 & SClara-82 & 164 & 633 & 0.7 & 0.4275 & 11.9 \\
\hline 06 & SClara-78 & 503 & 54729 & 9.8 & 3.6739 & 2.0 \\
\hline 06 & SClara-49 & 231 & 26796 & 1.0 & 4.7360 & 3.8 \\
\hline 06 & SClara-43 & 100 & 30030 & 2.9 & 4.6096 & 5.6 \\
\hline 06 & SClara-67 & 57 & 16833 & 0.7 & 5.2681 & 2.4 \\
\hline 06 & SClara-42 & 240 & 10506 & 2.1 & 5.0556 & 4.1 \\
\hline 06 & SClara-39 & 764 & 56106 & 4.0 & 8.5131 & 6.8 \\
\hline 06 & SClara-97 & 271 & 3504 & 0.7 & 9.5018 & 4.1 \\
\hline 06 & SClara-31 & 56 & 23331 & 0.8 & 13.7022 & 2.5 \\
\hline 07 & CR1-65 & 727 & 6872 & 1.2 & 0.10099 & 2.8 \\
\hline 07 & CR1-36 & 329 & 5307 & 0.5 & 0.16612 & 5.2 \\
\hline 07 & CR1-41 & 198 & 2563 & 0.2 & 0.18083 & 3.9 \\
\hline 07 & CR1-60 & 136 & 1341 & 0.3 & 0.18591 & 8.6 \\
\hline 07 & CR1-12 & 136 & 3095 & 0.6 & 0.28857 & 7.3 \\
\hline 07 & CR1-23 & 399 & 11126 & 1.8 & 0.33191 & 3.7 \\
\hline 07 & CR1-1 & 369 & 7848 & 1.7 & 0.37938 & 4.8 \\
\hline 07 & CR1-46 & 531 & 22805 & 1.2 & 0.46816 & 2.7 \\
\hline 07 & CR1-43 & 148 & 3872 & 0.5 & 0.46791 & 4.1 \\
\hline 07 & CR1-51 & 206 & 7085 & 1.2 & 0.49617 & 4.0 \\
\hline 07 & CR1-14 & 439 & 18475 & 0.5 & 0.50775 & 2.7 \\
\hline 07 & CR1-64 & 245 & 10399 & 0.7 & 0.51263 & 4.9 \\
\hline 07 & CR1-50 & 282 & 12526 & 0.5 & 0.52829 & 1.8 \\
\hline 07 & CR1-48 & 474 & 16135 & 1.4 & 0.57668 & 1.6 \\
\hline 07 & CR1-4 & 221 & 3984 & 0.5 & 0.59995 & 4.4 \\
\hline 07 & CR1-38 & 286 & 14934 & 0.7 & 0.75901 & 2.7 \\
\hline 07 & CR1-19 & 214 & 20291 & 0.7 & 0.88778 & 3.6 \\
\hline 07 & CR1-37 & 182 & 10229 & 0.5 & 1.18025 & 3.8 \\
\hline 07 & CR1-11 & 54 & 5392 & 1.6 & 1.51761 & 3.2 \\
\hline 07 & CR1-2 & 225 & 22868 & 0.9 & 1.69394 & 2.8 \\
\hline 07 & CR1-54 & 99 & 11140 & 0.9 & 1.75028 & 4.7 \\
\hline 07 & CR1-31 & 247 & 18997 & 2.1 & 1.81485 & 1.6 \\
\hline 07 & CR1-18 & 47 & 4600 & 1.1 & 1.72403 & 3.5 \\
\hline 07 & CR1-7 & 91 & 6719 & 0.6 & 1.39633 & 2.4 \\
\hline 07 & CR1-34 & 97 & 8577 & 0.5 & 1.88644 & 3.9 \\
\hline 07 & CR1-3 & 228 & 8175 & 2.2 & 1.77913 & 2.9 \\
\hline 07 & CR1-16 & 321 & 27629 & 1.5 & 1.80509 & 1.9 \\
\hline 07 & CR1-49 & 211 & 23312 & 0.3 & 1.77230 & 2.9 \\
\hline
\end{tabular}


Kimbrough, D.L., Grove, M., Gehrels, G.E., Dorsey, R.J., Howard, K.A., Lovera, O., Aslan, A., House, P.K., and Pearthree, P.A., 2015, Detrital zircon U-Pb provenance of the Colorado River: A 5 m.y. record of incision into cover strata overlying the Colorado Plateau and adjacent regions: Geosphere, v. 11, doi:10.1130/GES00982.1.

TABLE DR7. U-Pb ZIRCON LA-ICP-MS GEOCHRONOLOGIC ANALYSES OF COLORADO RIVER BASIN \& ASSOCIATED SAMPLES.

\begin{tabular}{|c|c|c|c|c|c|c|}
\hline & & & & & & opic ra \\
\hline ID\# & Analysis & $\begin{array}{c}\text { U } \\
(\mathrm{ppm})\end{array}$ & $\begin{array}{l}206 \mathrm{~Pb} \\
204 \mathrm{~Pb}\end{array}$ & U/Th & $\begin{array}{c}207 \mathrm{~Pb}^{*} \\
235 \mathrm{U}\end{array}$ & $\begin{array}{c} \pm \\
(\%)\end{array}$ \\
\hline 07 & CR1-27 & 173 & 3560 & 0.6 & 1.56631 & 3.0 \\
\hline 07 & CR1-47 & 262 & 20528 & 2.1 & 1.86341 & 3.5 \\
\hline 07 & CR1-17 & 92 & 12445 & 1.5 & 1.81412 & 4.9 \\
\hline 07 & CR1-44 & 196 & 19221 & 0.7 & 1.97386 & 3.8 \\
\hline 07 & CR1-45 & 638 & 79657 & 2.3 & 2.10072 & 3.1 \\
\hline 07 & CR1-55 & 93 & 7908 & 1.3 & 2.04265 & 2.1 \\
\hline 07 & CR1-62 & 58 & 5887 & 1.2 & 1.89526 & 5.5 \\
\hline 07 & CR1-39 & 255 & 17822 & 1.4 & 2.09184 & 4.8 \\
\hline 07 & CR1-8 & 11 & 1985 & 0.7 & 2.51074 & 6.2 \\
\hline 07 & CR1-56 & 293 & 21966 & 0.9 & 2.51774 & 3.2 \\
\hline 07 & CR1-32 & 157 & 14849 & 1.0 & 2.68216 & 3.6 \\
\hline 07 & CR1-33 & 46 & 1000 & 0.8 & 2.25669 & 6.1 \\
\hline 07 & CR1-59 & 83 & 1805 & 0.6 & 1.77337 & 17.4 \\
\hline 07 & CR1-63 & 286 & 9521 & 0.9 & 2.58754 & 2.1 \\
\hline 07 & CR1-53 & 171 & 34037 & 1.3 & 3.26115 & 1.9 \\
\hline 07 & CR1-5 & 99 & 17423 & 0.7 & 4.12287 & 3.4 \\
\hline 07 & CR1-20 & 126 & 24049 & 0.8 & 3.90741 & 4.0 \\
\hline 07 & CR1-52 & 838 & 124354 & 4.6 & 4.04407 & 2.8 \\
\hline 07 & CR1-28 & 146 & 29741 & 0.9 & 4.27081 & 2.1 \\
\hline 07 & CR1-61 & 515 & 14871 & 1.6 & 4.16297 & 3.2 \\
\hline 07 & CR1-26 & 120 & 28304 & 0.6 & 4.43429 & 3.9 \\
\hline 07 & CR1-21 & 283 & 51081 & 0.4 & 4.63387 & 1.8 \\
\hline 07 & CR1-22 & 300 & 41159 & 1.1 & 4.58680 & 3.7 \\
\hline 07 & CR1-35 & 199 & 37633 & 1.3 & 4.90835 & 1.9 \\
\hline 07 & CR1-29 & 275 & 13558 & 1.2 & 5.22212 & 4.0 \\
\hline 07 & CR1-30 & 126 & 23897 & 0.6 & 6.82448 & 2.2 \\
\hline 07 & CR1-40 & 140 & 26285 & 0.3 & 6.71863 & 2.1 \\
\hline 07 & CR1-25 & 26 & 178 & 2.0 & 7.02123 & 5.0 \\
\hline 07 & CR1-24 & 33 & 7540 & 0.4 & 6.80974 & 5.5 \\
\hline 07 & CR1-57 & 74 & 22077 & 0.6 & 13.92396 & 3.5 \\
\hline 07 & CR1-6 & 186 & 43794 & 0.5 & 14.22394 & 3.0 \\
\hline 08 & CR2-1 & 1672 & 1692 & 0.2 & 0.0730 & 22.6 \\
\hline 08 & CR2-50 & 244 & 2282 & 1.3 & 0.0915 & 6.4 \\
\hline 08 & CR2-14 & 584 & 614 & 0.7 & 0.2731 & 10.1 \\
\hline 08 & CR2-51 & 534 & 7810 & 1.3 & 0.1723 & 6.5 \\
\hline 08 & CR2-15 & 471 & 5420 & 0.8 & 0.1855 & 3.3 \\
\hline 08 & CR2-74 & 241 & 4516 & 1.0 & 0.1845 & 4.9 \\
\hline 08 & CR2-69 & 567 & 9430 & 1.1 & 0.1888 & 4.2 \\
\hline 08 & CR2-43 & 693 & 5710 & 1.2 & 0.2595 & 3.5 \\
\hline 08 & CR2-73 & 319 & 2276 & 0.6 & 0.4240 & 10.1 \\
\hline 08 & CR2-72 & 64 & 4162 & 1.2 & 0.4234 & 5.3 \\
\hline 08 & CR2-17 & 90 & 1512 & 1.0 & 0.5523 & 18.6 \\
\hline 08 & CR2-59 & 232 & 15252 & 1.4 & 0.4776 & 4.4 \\
\hline 08 & CR2-11 & 140 & 3502 & 1.3 & 0.5406 & 3.0 \\
\hline 08 & CR2-12 & 247 & 4592 & 0.9 & 0.5885 & 6.4 \\
\hline 08 & CR2-37 & 330 & 8868 & 1.1 & 0.5646 & 3.2 \\
\hline 08 & CR2-5 & 36 & 1742 & 0.4 & 0.6859 & 7.3 \\
\hline 08 & CR2-25 & 142 & 8922 & 1.0 & 0.7702 & 3.5 \\
\hline 08 & CR2-62 & 269 & 5616 & 0.6 & 0.8394 & 3.5 \\
\hline 08 & CR2-66 & 131 & 10616 & 0.9 & 0.8047 & 4.8 \\
\hline 08 & CR2-23 & 374 & 5202 & 0.8 & 0.8708 & 2.5 \\
\hline 08 & CR2-3 & 97 & 5406 & 2.3 & 1.0272 & 2.5 \\
\hline 08 & CR2-20 & 105 & 4934 & 1.2 & 1.6036 & 5.2 \\
\hline 08 & CR2-22 & 44 & 2664 & 1.1 & 1.7693 & 2.1 \\
\hline 08 & CR2-33 & 183 & 25452 & 3.1 & 1.7761 & 4.3 \\
\hline 08 & CR2-9 & 362 & 47778 & 2.2 & 2.0472 & 2.3 \\
\hline 08 & CR2-47 & 64 & 8744 & 3.8 & 2.0922 & 4.3 \\
\hline 08 & CR2-28 & 256 & 21560 & 1.8 & 1.9691 & 2.4 \\
\hline 08 & CR2-54 & 58 & 10034 & 1.4 & 2.2888 & 2.8 \\
\hline 08 & CR2-29 & 59 & 10408 & 1.6 & 2.3259 & 2.8 \\
\hline 08 & CR2-44 & 335 & 4306 & 1.6 & 1.7748 & 3.4 \\
\hline 08 & CR2-63 & 385 & 21812 & 1.2 & 2.3263 & 2.7 \\
\hline 08 & CR2-61 & 205 & 40288 & 2.8 & 2.6646 & 5.1 \\
\hline 08 & CR2-60 & 405 & 15192 & 0.7 & 2.7619 & 4.2 \\
\hline 08 & CR2-46 & 48 & 8834 & 1.5 & 2.9036 & 3.8 \\
\hline 08 & CR2-10 & 53 & 13578 & 2.5 & 2.7478 & 2.0 \\
\hline 08 & CR2-68 & 48 & 8882 & 0.8 & 2.9576 & 2.0 \\
\hline 08 & CR2-31 & 18 & 3666 & 1.3 & 2.9273 & 3.7 \\
\hline 08 & CR2-18 & 290 & 6500 & 0.8 & 2.5352 & 4.7 \\
\hline 08 & CR2-67 & 247 & 5208 & 3.4 & 2.6028 & 5.1 \\
\hline 08 & CR2-55 & 117 & 14466 & 2.0 & 2.8757 & 3.8 \\
\hline 08 & CR2-75 & 91 & 26488 & 1.6 & 3.4242 & 2.3 \\
\hline 08 & CR2-65 & 220 & 41726 & 1.9 & 3.3183 & 2.3 \\
\hline 08 & CR2-40 & 530 & 72574 & 2.0 & 3.5452 & 2.2 \\
\hline 08 & CR2-34 & 130 & 20910 & 2.4 & 3.5583 & 3.5 \\
\hline 08 & CR2-52 & 108 & 20548 & 2.0 & 3.6875 & 1.8 \\
\hline 08 & CR2-35 & 34 & 11460 & 1.4 & 3.7737 & 3.0 \\
\hline 08 & CR2-32 & 55 & 13506 & 0.8 & 4.0866 & 4.3 \\
\hline 08 & CR2-38 & 1109 & 31708 & 1.8 & 3.0489 & 5.9 \\
\hline 08 & CR2-71 & 279 & 44238 & 2.7 & 4.6029 & 3.7 \\
\hline 08 & CR2-30 & 320 & 834 & 0.4 & 1.4494 & 3.2 \\
\hline 08 & CR2-16 & 341 & 11000 & 1.7 & 3.4025 & 3.1 \\
\hline 08 & CR2-57 & 103 & 26272 & 2.0 & 4.7128 & 3.3 \\
\hline 08 & CR2-39 & 197 & 13378 & 1.5 & 4.7342 & 2.8 \\
\hline 08 & CR2-8 & 66 & 9778 & 0.9 & 5.0763 & 3.1 \\
\hline 08 & CR2-36 & 497 & 33640 & 3.4 & 4.8219 & 2.9 \\
\hline 08 & CR2-7 & 105 & 2684 & 0.6 & 4.4240 & 3.4 \\
\hline 08 & CR2-27 & 73 & 9976 & 1.0 & 5.0559 & 2.6 \\
\hline 08 & CR2-45 & 153 & 17400 & 1.1 & 5.7721 & 1.7 \\
\hline 08 & CR2-42 & 104 & 28166 & 1.9 & 5.8438 & 3.8 \\
\hline
\end{tabular}


Kimbrough, D.L., Grove, M., Gehrels, G.E., Dorsey, R.J., Howard, K.A., Lovera, O., Aslan, A., House, P.K., and Pearthree, P.A., 2015, Detrital zircon U-Pb provenance of the Colorado River: A 5 m.y. record of incision into cover strata overlying the Colorado Plateau and adjacent regions: Geosphere, v. 11, doi:10.1130/GES00982.1.

TABLE DR7. U-Pb ZIRCON LA-ICP-MS GEOCHRONOLOGIC ANALYSES OF COLORADO RIVER BASIN \& ASSOCIATED SAMPLES.

\begin{tabular}{|c|c|c|c|c|c|c|}
\hline & & & & & & opic ra \\
\hline ID\# & Analysis & $\begin{array}{c}U \\
(\mathrm{ppm})\end{array}$ & $\begin{array}{l}206 \mathrm{~Pb} \\
204 \mathrm{~Pb} \\
\end{array}$ & U/Th & $\begin{array}{c}207 \mathrm{~Pb}^{*} \\
235 \mathrm{U}\end{array}$ & $\begin{array}{c} \pm \\
(\%) \\
\end{array}$ \\
\hline 08 & CR2-56 & 238 & 77814 & 1.2 & 6.0536 & 4.0 \\
\hline 08 & CR2-24 & 213 & 44082 & 0.7 & 12.2615 & 2.4 \\
\hline 08 & CR2-58 & 212 & 31358 & 1.8 & 10.7731 & 6.2 \\
\hline 08 & CR2-4 & 318 & 144230 & 13.7 & 14.0606 & 2.9 \\
\hline 08 & CR2-41 & 125 & 47136 & 1.0 & 13.8587 & 2.8 \\
\hline 08 & CR2-2 & 139 & 38146 & 1.5 & 14.4113 & 1.6 \\
\hline 09 & CR3-37 & 122 & 496 & 0.6 & 0.0223 & 24.1 \\
\hline 09 & CR3-73 & 584 & 1000 & 1.1 & 0.1378 & 4.3 \\
\hline 09 & CR3-12 & 601 & 1828 & 0.7 & 0.2022 & 5.8 \\
\hline 09 & CR3-34 & 342 & 3826 & 1.3 & 0.1803 & 3.7 \\
\hline 09 & CR3-10 & 350 & 5102 & 1.3 & 0.1747 & 1.9 \\
\hline 09 & CR3-17 & 479 & 13348 & 1.1 & 0.1755 & 3.0 \\
\hline 09 & CR3-44 & 74 & 2268 & 1.8 & 0.2021 & 12.9 \\
\hline 09 & CR3-47 & 75 & 2274 & 1.7 & 0.2112 & 10.4 \\
\hline 09 & CR3-46 & 104 & 5756 & 1.4 & 0.2281 & 7.8 \\
\hline 09 & CR3-50 & 526 & 19104 & 1.6 & 0.2464 & 3.6 \\
\hline 09 & CR3-69 & 313 & 3910 & 0.5 & 0.2635 & 8.4 \\
\hline 09 & CR3-22 & 795 & 4012 & 1.6 & 0.4518 & 4.4 \\
\hline 09 & CR3-25 & 257 & 10382 & 1.7 & 0.4209 & 2.7 \\
\hline 09 & CR3-16 & 30 & 1444 & 1.1 & 0.4215 & 10.8 \\
\hline 09 & CR3-54 & 176 & 7730 & 2.2 & 0.4809 & 3.1 \\
\hline 09 & CR3-7 & 143 & 3942 & 3.4 & 0.4837 & 2.8 \\
\hline 09 & CR3-60 & 216 & 14934 & 2.0 & 0.4855 & 3.2 \\
\hline 09 & CR3-29 & 97 & 4456 & 0.8 & 0.5151 & 4.0 \\
\hline 09 & CR3-75 & 171 & 8644 & 0.8 & 0.5233 & 2.3 \\
\hline 09 & CR3-53 & 534 & 6166 & 2.2 & 0.6766 & 2.6 \\
\hline 09 & CR3-3 & 119 & 8854 & 1.3 & 0.7680 & 3.2 \\
\hline 09 & CR3-39 & 138 & 5396 & 0.6 & 0.8288 & 3.1 \\
\hline 09 & CR3-6 & 274 & 12234 & 3.4 & 1.0204 & 6.3 \\
\hline 09 & CR3-19 & 136 & 16646 & 2.0 & 1.4359 & 3.5 \\
\hline 09 & CR3-68 & 43 & 2550 & 1.3 & 1.5287 & 3.1 \\
\hline 09 & CR3-26 & 67 & 8294 & 1.5 & 1.7377 & 2.2 \\
\hline 09 & CR3-72 & 205 & 12906 & 2.1 & 1.8175 & 2.5 \\
\hline 09 & CR3-28 & 86 & 12126 & 2.2 & 2.0533 & 2.0 \\
\hline 09 & CR3-20 & 78 & 12390 & 1.7 & 2.0232 & 1.8 \\
\hline 09 & CR3-40 & 62 & 3442 & 1.0 & 1.8298 & 3.4 \\
\hline 09 & CR3-15 & 140 & 11488 & 2.0 & 2.0313 & 2.9 \\
\hline 09 & CR3-64 & 201 & 5936 & 1.7 & 2.0357 & 3.8 \\
\hline 09 & CR3-18 & 204 & 37570 & 3.7 & 2.2543 & 4.1 \\
\hline 09 & CR3-58 & 62 & 20684 & 1.7 & 2.5141 & 2.8 \\
\hline 09 & CR3-51 & 272 & 47414 & 3.4 & 2.7533 & 2.1 \\
\hline 09 & CR3-24 & 106 & 13368 & 1.4 & 2.6691 & 2.3 \\
\hline 09 & CR3-36 & 235 & 36826 & 2.6 & 2.7820 & 3.1 \\
\hline 09 & CR3-45 & 85 & 8104 & 3.0 & 2.7201 & 5.1 \\
\hline 09 & CR3-38 & 378 & 25304 & 1.0 & 2.7724 & 2.5 \\
\hline 09 & CR3-33 & 482 & 7668 & 1.9 & 2.7592 & 3.1 \\
\hline 09 & CR3-8 & 267 & 44924 & 2.8 & 3.2099 & 1.5 \\
\hline 09 & CR3-49 & 147 & 51230 & 2.3 & 2.8974 & 2.9 \\
\hline 09 & CR3-74 & 68 & 9554 & 0.9 & 3.0029 & 2.0 \\
\hline 09 & CR3-43 & 220 & 64850 & 1.8 & 2.9512 & 4.1 \\
\hline 09 & CR3-55 & 101 & 27176 & 1.3 & 3.3131 & 3.5 \\
\hline 09 & CR3-14 & 125 & 18182 & 1.9 & 3.4084 & 1.9 \\
\hline 09 & CR3-66 & 126 & 22492 & 0.7 & 3.7133 & 1.9 \\
\hline 09 & CR3-2 & 91 & 15978 & 1.6 & 3.9527 & 2.2 \\
\hline 09 & CR3-57 & 56 & 18652 & 2.0 & 3.9206 & 3.2 \\
\hline 09 & CR3-67 & 135 & 28218 & 1.6 & 4.3130 & 2.0 \\
\hline 09 & CR3-52 & 656 & 107404 & 2.4 & 4.3181 & 3.6 \\
\hline 09 & CR3-23 & 536 & 49330 & 5.4 & 4.1294 & 2.4 \\
\hline 09 & CR3-5 & 114 & 14584 & 3.3 & 4.6450 & 2.7 \\
\hline 09 & CR3-59 & 234 & 3348 & 2.7 & 3.3543 & 3.0 \\
\hline 09 & CR3-21 & 378 & 4808 & 1.8 & 3.6181 & 3.1 \\
\hline 09 & CR3-41 & 114 & 21908 & 2.1 & 4.2636 & 4.2 \\
\hline 09 & CR3-70 & 88 & 11812 & 1.2 & 4.0242 & 4.7 \\
\hline 09 & CR3-9 & 83 & 12906 & 1.8 & 4.7281 & 2.3 \\
\hline 09 & CR3-32 & 236 & 36276 & 2.4 & 4.5172 & 1.4 \\
\hline 09 & CR3-71 & 147 & 9566 & 2.0 & 4.2976 & 5.0 \\
\hline 09 & CR3-30 & 160 & 31242 & 1.1 & 5.5613 & 2.5 \\
\hline 09 & CR3-56 & 128 & 21304 & 3.4 & 4.9936 & 4.5 \\
\hline 09 & CR3-61 & 35 & 12354 & 1.5 & 6.6675 & 3.3 \\
\hline 09 & CR3-27 & 493 & 58474 & 1.6 & 10.7971 & 3.5 \\
\hline 09 & CR3-31 & 110 & 39760 & 0.6 & 13.7400 & 2.4 \\
\hline 10 & CRT0806-1-43 & 647 & 1817 & 1.9 & 0.09191 & 10.4 \\
\hline 10 & CRT0806-1-53 & 907 & 6954 & 2.1 & 0.11325 & 4.5 \\
\hline 10 & CRT0806-1-34 & 286 & 6956 & 1.8 & 0.30467 & 2.6 \\
\hline 10 & CRT0806-1-45 & 191 & 3355 & 1.1 & 0.34439 & 4.6 \\
\hline 10 & CRT0806-1-26 & 518 & 10008 & 2.5 & 0.38541 & 1.7 \\
\hline 10 & CRT0806-1-32 & 590 & 10560 & 5.8 & 0.41702 & 2.1 \\
\hline 10 & CRT0806-1-51 & 190 & 3942 & 1.7 & 0.49490 & 3.3 \\
\hline 10 & CRT0806-1-09 & 375 & 15980 & 1.6 & 0.81105 & 1.8 \\
\hline 10 & CRT0806-1-14 & 193 & 8730 & 1.0 & 0.83844 & 3.2 \\
\hline 10 & CRT0806-1-30 & 173 & 9870 & 1.3 & 0.88608 & 2.3 \\
\hline 10 & CRT0806-1-24 & 309 & 10531 & 1.2 & 1.55844 & 2.2 \\
\hline 10 & CRT0806-1-2 & 264 & 24464 & 2.4 & 1.63707 & 6.9 \\
\hline 10 & CRT0806-1-29 & 133 & 5623 & 1.4 & 1.79106 & 2.1 \\
\hline 10 & CRT0806-1-11 & 281 & 10799 & 2.6 & 1.88801 & 2.5 \\
\hline 10 & CRT0806-1-54 & 122 & 9917 & 3.2 & 1.93294 & 2.7 \\
\hline 10 & CRT0806-1-27 & 494 & 37836 & 2.5 & 2.06108 & 2.2 \\
\hline 10 & CRT0806-1-36 & 287 & 24898 & 2.7 & 2.06971 & 2.3 \\
\hline 10 & CRT0806-1-18 & 446 & 15851 & 2.5 & 1.91615 & 5.8 \\
\hline 10 & CRT0806-1-48 & 111 & 6269 & 3.3 & 2.11827 & 6.5 \\
\hline
\end{tabular}


Kimbrough, D.L., Grove, M., Gehrels, G.E., Dorsey, R.J., Howard, K.A., Lovera, O., Aslan, A., House, P.K., and Pearthree, P.A., 2015, Detrital zircon U-Pb provenance of the Colorado River: A 5 m.y. record of incision into cover strata overlying the Colorado Plateau and adjacent regions: Geosphere, v. 11, doi:10.1130/GES00982.1.

TABLE DR7. U-Pb ZIRCON LA-ICP-MS GEOCHRONOLOGIC ANALYSES OF COLORADO RIVER BASIN \& ASSOCIATED SAMPLES.

\begin{tabular}{|c|c|c|c|c|c|c|}
\hline & & & & & & opic ra \\
\hline ID\# & Analysis & $\begin{array}{c}\text { U } \\
(\mathrm{ppm})\end{array}$ & $\begin{array}{l}206 \mathrm{~Pb} \\
204 \mathrm{~Pb}\end{array}$ & $\mathrm{U} / \mathrm{Th}$ & $\begin{array}{c}207 \mathrm{~Pb}^{*} \\
235 \mathrm{U}\end{array}$ & $\begin{array}{c} \pm \\
(\%)\end{array}$ \\
\hline 10 & CRT0806-1-44 & 254 & 13678 & 2.9 & 2.11948 & 1.6 \\
\hline 10 & CRT0806-1-6 & 51 & 8555 & 1.1 & 1.94727 & 6.4 \\
\hline 10 & CRT0806-1-21 & 260 & 13233 & 1.7 & 2.31310 & 3.1 \\
\hline 10 & CRT0806-1-39 & 169 & 11583 & 1.8 & 2.47334 & 4.4 \\
\hline 10 & CRT0806-1-49 & 411 & 14774 & 2.8 & 1.91770 & 5.2 \\
\hline 10 & CRT0806-1-23 & 47 & 1749 & 0.8 & 2.10314 & 2.3 \\
\hline 10 & CRT0806-1-50 & 108 & 3895 & 0.6 & 2.76624 & 3.9 \\
\hline 10 & CRT0806-1-4 & 57 & 7052 & 1.2 & 2.84471 & 6.3 \\
\hline 10 & CRT0806-1-3 & 122 & 17774 & 1.1 & 3.01810 & 7.1 \\
\hline 10 & CRT0806-1-20 & 632 & 51696 & 4.4 & 2.89455 & 4.4 \\
\hline 10 & CRT0806-1-58 & 217 & 46965 & 2.1 & 3.14987 & 4.3 \\
\hline 10 & CRT0806-1-40 & 305 & 7182 & 1.1 & 2.78541 & 3.5 \\
\hline 10 & CRT0806-1-28 & 274 & 34231 & 2.1 & 3.11419 & 1.8 \\
\hline 10 & CRT0806-1-55 & 52 & 5101 & 1.1 & 3.27776 & 5.2 \\
\hline 10 & CRT0806-1-31 & 106 & 16339 & 1.3 & 3.47871 & 3.1 \\
\hline 10 & CRT0806-1-46 & 206 & 19788 & 2.5 & 3.38139 & 2.9 \\
\hline 10 & CRT0806-1-47 & 1463 & 2574 & 1.8 & 1.97477 & 4.9 \\
\hline 10 & CRT0806-1-15 & 184 & 12380 & 1.2 & 3.75499 & 3.0 \\
\hline 10 & CRT0806-1-25 & 2176 & 23357 & 13.2 & 3.73257 & 6.5 \\
\hline 10 & CRT0806-1-5 & 129 & 24142 & 1.7 & 4.15907 & 6.3 \\
\hline 10 & CRT0806-1-22 & 106 & 13885 & 1.8 & 4.14132 & 2.0 \\
\hline 10 & CRT0806-1-35 & 573 & 62410 & 6.4 & 4.15912 & 1.9 \\
\hline 10 & CRT0806-1-57 & 948 & 8260 & 2.2 & 3.89410 & 7.8 \\
\hline 10 & CRT0806-1-37 & 169 & 6049 & 1.8 & 4.25583 & 4.9 \\
\hline 10 & CRT0806-1-56 & 363 & 13644 & 3.0 & 4.06773 & 5.9 \\
\hline 10 & CRT0806-1-52 & 229 & 23731 & 1.1 & 4.55156 & 3.3 \\
\hline 10 & CRT0806-1-1 & 258 & 30840 & 1.9 & 4.50356 & 7.1 \\
\hline 10 & CRT0806-1-17 & 172 & 18177 & 5.5 & 4.35659 & 2.6 \\
\hline 10 & CRT0806-1-13 & 697 & 51603 & 2.4 & 4.58055 & 4.0 \\
\hline 10 & CRT0806-1-42 & 300 & 16007 & 2.1 & 4.67034 & 3.1 \\
\hline 10 & CRT0806-1-38 & 262 & 36630 & 1.9 & 4.68819 & 2.8 \\
\hline 10 & CRT0806-1-7 & 563 & 6368 & 2.5 & 4.90100 & 4.6 \\
\hline 10 & CRT0806-1-16 & 137 & 8743 & 1.2 & 4.92274 & 2.0 \\
\hline 10 & CRT0806-1-33 & 107 & 15985 & 0.7 & 6.07540 & 2.3 \\
\hline 10 & CRT0806-1-60 & 246 & 24890 & 3.0 & 7.67880 & 4.7 \\
\hline 10 & CRT0806-1-10 & 174 & 42604 & 3.2 & 15.84275 & 1.9 \\
\hline 10 & CRT0806-1-12 & 135 & 28561 & 1.5 & 15.96375 & 5.2 \\
\hline 11 & CRT0806-3-50 & 381 & 4516 & 5.6 & 0.21457 & 3.7 \\
\hline 11 & CRT0806-3-39 & 424 & 13527 & 1.8 & 0.49153 & 2.3 \\
\hline 11 & CRT0806-3-53 & 866 & 13685 & 5.1 & 0.54629 & 3.2 \\
\hline 11 & CRT0806-3-14 & 351 & 9594 & 2.6 & 0.54824 & 1.9 \\
\hline 11 & CRT0806-3-44 & 212 & 5042 & 0.9 & 0.54354 & 2.5 \\
\hline 11 & CRT0806-3-32 & 232 & 10549 & 3.5 & 0.60775 & 2.2 \\
\hline 11 & CRT0806-3-13 & 522 & 15664 & 3.6 & 0.68333 & 3.0 \\
\hline 11 & CRT0806-3-46 & 273 & 4356 & 2.5 & 0.70796 & 7.4 \\
\hline 11 & CRT0806-3-22 & 110 & 5946 & 7.4 & 1.15761 & 2.2 \\
\hline 11 & CRT0806-3-23 & 264 & 23156 & 1.7 & 1.74663 & 1.9 \\
\hline 11 & CRT0806-3-5 & 736 & 47541 & 6.0 & 1.70996 & 1.9 \\
\hline 11 & CRT0806-3-11 & 404 & 19112 & 2.1 & 1.71497 & 2.7 \\
\hline 11 & CRT0806-3-35 & 301 & 30232 & 3.0 & 1.75139 & 2.0 \\
\hline 11 & CRT0806-3-34 & 124 & 7024 & 1.8 & 1.82825 & 1.9 \\
\hline 11 & CRT0806-3-45 & 217 & 16894 & 1.1 & 1.86231 & 2.0 \\
\hline 11 & CRT0806-3-38 & 267 & 25351 & 3.2 & 1.87748 & 1.4 \\
\hline 11 & CRT0806-3-33 & 177 & 17852 & 2.4 & 1.85497 & 3.2 \\
\hline 11 & CRT0806-3-27 & 84 & 8677 & 1.7 & 2.07882 & 2.4 \\
\hline 11 & CRT0806-3-58 & 399 & 17464 & 2.3 & 2.01655 & 2.5 \\
\hline 11 & CRT0806-3-2 & 39 & 4392 & 1.9 & 2.20876 & 2.5 \\
\hline 11 & CRT0806-3-52 & 112 & 9695 & 1.9 & 2.63443 & 1.7 \\
\hline 11 & CRT0806-3-15 & 133 & 14772 & 2.0 & 3.02312 & 1.9 \\
\hline 11 & CRT0806-3-16 & 182 & 21370 & 3.0 & 3.00030 & 2.1 \\
\hline 11 & CRT0806-3-17 & 120 & 13588 & 2.0 & 3.01331 & 1.9 \\
\hline 11 & CRT0806-3-31 & 109 & 13582 & 1.2 & 3.02526 & 2.2 \\
\hline 11 & CRT0806-3-18 & 106 & 14598 & 1.3 & 3.02292 & 1.8 \\
\hline 11 & CRT0806-3-4 & 111 & 12749 & 0.9 & 2.96082 & 2.3 \\
\hline 11 & CRT0806-3-19 & 193 & 19060 & 2.1 & 3.02886 & 1.6 \\
\hline 11 & CRT0806-3-10 & 181 & 23859 & 3.6 & 2.98459 & 1.8 \\
\hline 11 & CRT0806-3-8 & 177 & 25091 & 1.4 & 3.20335 & 3.8 \\
\hline 11 & CRT0806-3-29 & 244 & 36881 & 1.9 & 3.03695 & 1.8 \\
\hline 11 & CRT0806-3-36 & 88 & 7926 & 1.0 & 3.10984 & 1.5 \\
\hline 11 & CRT0806-3-6 & 283 & 31329 & 3.0 & 3.09175 & 3.4 \\
\hline 11 & CRT0806-3-3 & 159 & 15950 & 2.5 & 3.05620 & 1.8 \\
\hline 11 & CRT0806-3-47 & 297 & 35270 & 2.6 & 3.17813 & 2.0 \\
\hline 11 & CRT0806-3-7 & 167 & 27070 & 2.5 & 3.02416 & 2.1 \\
\hline 11 & CRT0806-3-48 & 157 & 18990 & 2.0 & 3.61122 & 1.5 \\
\hline 11 & CRT0806-3-25 & 358 & 53774 & 3.8 & 3.93709 & 1.5 \\
\hline 11 & CRT0806-3-59 & 106 & 15229 & 1.9 & 3.99634 & 3.8 \\
\hline 11 & CRT0806-3-30 & 442 & 45234 & 6.9 & 4.16193 & 2.1 \\
\hline 11 & CRT0806-3-26 & 135 & 14367 & 0.9 & 4.20190 & 1.9 \\
\hline 11 & CRT0806-3-37 & 242 & 32491 & 2.5 & 4.56564 & 3.2 \\
\hline 11 & CRT0806-3-24 & 229 & 24605 & 2.0 & 4.32133 & 1.8 \\
\hline 11 & CRT0806-3-56 & 437 & 42516 & 1.0 & 4.55287 & 2.3 \\
\hline 11 & CRT0806-3-20 & 367 & 43312 & 1.4 & 4.49823 & 1.6 \\
\hline 11 & CRT0806-3-9 & 312 & 19556 & 2.6 & 4.46535 & 2.2 \\
\hline 11 & CRT0806-3-43 & 431 & 36918 & 2.8 & 4.51335 & 4.4 \\
\hline 11 & CRT0806-3-40 & 56 & 2821 & 0.7 & 4.57996 & 2.7 \\
\hline 11 & CRT0806-3-60 & 351 & 24189 & 2.0 & 4.49715 & 2.7 \\
\hline 11 & CRT0806-3-12 & 44 & 2229 & 3.5 & 4.41602 & 4.1 \\
\hline 11 & CRT0806-3-55 & 157 & 51098 & 2.8 & 5.36351 & 1.5 \\
\hline 11 & CRT0806-3-49 & 111 & 21452 & 1.7 & 10.83985 & 1.8 \\
\hline 11 & CRT0806-3-51 & 257 & 49348 & 1.2 & 34.78745 & 2.4 \\
\hline
\end{tabular}


Kimbrough, D.L., Grove, M., Gehrels, G.E., Dorsey, R.J., Howard, K.A., Lovera, O., Aslan, A., House, P.K., and Pearthree, P.A., 2015, Detrital zircon U-Pb provenance of the Colorado River: A 5 m.y. record of incision into cover strata overlying the Colorado Plateau and adjacent regions: Geosphere, v. 11, doi:10.1130/GES00982.1.

TABLE DR7. U-Pb ZIRCON LA-ICP-MS GEOCHRONOLOGIC ANALYSES OF COLORADO RIVER BASIN \& ASSOCIATED SAMPLES.

\begin{tabular}{|c|c|c|c|c|c|c|}
\hline & & & & & & opic ra \\
\hline ID\# & Analysis & $\begin{array}{c}U \\
(\mathrm{ppm})\end{array}$ & $\begin{array}{l}206 \mathrm{~Pb} \\
204 \mathrm{~Pb}\end{array}$ & $\mathrm{U} / \mathrm{Th}$ & $\begin{array}{c}207 \mathrm{~Pb}^{*} \\
235 \mathrm{U}\end{array}$ & $\begin{array}{c} \pm \\
(\%) \\
\end{array}$ \\
\hline 12 & CRT0806-2-13 & 653 & 102 & 1.2 & 0.04306 & 15.3 \\
\hline 12 & CRT08062-27 & 441 & 909 & 0.7 & 0.03686 & 15.6 \\
\hline 12 & CRT08062-65 & 870 & 2120 & 0.7 & 0.04996 & 4.6 \\
\hline 12 & CRT0806-2-1 & 1942 & 1954 & 0.9 & 0.04833 & 11.0 \\
\hline 12 & CRT0806-2-26 & 927 & 1913 & 0.2 & 0.04078 & 8.9 \\
\hline 12 & CRT08062-2 & 355 & 1395 & 0.5 & 0.07775 & 6.6 \\
\hline 12 & CRT08062-58 & 939 & 4287 & 1.1 & 0.09398 & 4.0 \\
\hline 12 & CRT08062-61 & 382 & 3461 & 0.9 & 0.09640 & 7.0 \\
\hline 12 & CRT08062-54 & 809 & 4665 & 0.9 & 0.10791 & 5.7 \\
\hline 12 & CRT0806-2-52 & 805 & 6022 & 1.1 & 0.10278 & 4.5 \\
\hline 12 & CRT0806-2-61 & 1052 & 2978 & 0.9 & 0.10278 & 6.6 \\
\hline 12 & CRT0806-2-31 & 1526 & 15093 & 0.8 & 0.17316 & 1.6 \\
\hline 12 & CRT08062-59 & 639 & 8249 & 0.6 & 0.17561 & 4.8 \\
\hline 12 & CRT0806-2-48 & 1736 & 9190 & 0.7 & 0.17267 & 3.3 \\
\hline 12 & CRT08062-1 & 1069 & 4897 & 0.5 & 0.18715 & 4.0 \\
\hline 12 & CRT0806-2-25 & 1251 & 1443 & 0.9 & 0.19230 & 14.6 \\
\hline 12 & CRT0806-2-44 & 825 & 8125 & 1.7 & 0.24944 & 5.8 \\
\hline 12 & CRT08062-20 & 454 & 8500 & 0.8 & 0.26718 & 3.1 \\
\hline 12 & CRT0806-2-47 & 607 & 10407 & 1.2 & 0.39156 & 3.5 \\
\hline 12 & CRT08062-4 & 355 & 11538 & 0.9 & 0.42496 & 4.0 \\
\hline 12 & CRT08062-29 & 103 & 4246 & 0.4 & 0.45634 & 6.8 \\
\hline 12 & CRT0806-2-64 & 266 & 419 & 1.0 & 0.23993 & 56.1 \\
\hline 12 & CRT08062-34 & 1008 & 3618 & 17.4 & 0.52871 & 3.7 \\
\hline 12 & CRT08062-3 & 221 & 9706 & 0.8 & 0.51657 & 5.0 \\
\hline 12 & CRT08062-64 & 397 & 15481 & 0.7 & 0.52797 & 1.5 \\
\hline 12 & CRT08062-7 & 249 & 5052 & 0.9 & 0.52914 & 3.8 \\
\hline 12 & CRT08062-23 & 716 & 6717 & 2.1 & 0.53838 & 3.6 \\
\hline 12 & CRT0806-2-63 & 220 & 5722 & 7.7 & 0.65423 & 8.9 \\
\hline 12 & CRT08062-10 & 219 & 891 & 0.9 & 0.65310 & 4.7 \\
\hline 12 & CRT08062-52 & 178 & 8492 & 2.2 & 0.62430 & 4.8 \\
\hline 12 & CRT08062-45 & 95 & 6786 & 0.8 & 0.71558 & 5.5 \\
\hline 12 & CRT08062-22 & 71 & 2209 & 0.5 & 0.81034 & 4.9 \\
\hline 12 & CRT08062-35 & 373 & 36433 & 0.7 & 0.76422 & 2.9 \\
\hline 12 & CRT08062-26 & 209 & 9355 & 0.5 & 0.77840 & 3.9 \\
\hline 12 & CRT08062-5 & 128 & 2038 & 0.4 & 0.80622 & 4.2 \\
\hline 12 & CRT08062-13 & 120 & 9074 & 0.4 & 0.83035 & 3.6 \\
\hline 12 & CRT08062-63 & 135 & 9631 & 0.6 & 0.89652 & 4.3 \\
\hline 12 & CRT0806-2-29 & 388 & 9094 & 0.7 & 0.90223 & 4.3 \\
\hline 12 & CRT0806-2-36 & 203 & 9034 & 1.0 & 0.99068 & 6.6 \\
\hline 12 & CRT0806-2-58 & 161 & 7805 & 0.8 & 1.35402 & 9.8 \\
\hline 12 & CRT0806-2-45 & 120 & 1962 & 0.8 & 1.55256 & 8.5 \\
\hline 12 & CRT0806-2-46 & 267 & 10930 & 1.1 & 1.48431 & 2.9 \\
\hline 12 & CRT0806-2-23 & 294 & 12950 & 1.4 & 1.55804 & 4.1 \\
\hline 12 & CRT08062-53 & 43 & 5483 & 0.6 & 1.61094 & 7.0 \\
\hline 12 & CRT08062-46 & 328 & 32351 & 1.7 & 1.62135 & 2.0 \\
\hline 12 & CRT0806-2-34 & 372 & 33222 & 1.6 & 1.69074 & 1.9 \\
\hline 12 & CRT0806-2-14 & 263 & 8505 & 0.4 & 1.99504 & 5.5 \\
\hline 12 & CRT08062-36 & 91 & 11379 & 0.4 & 1.87022 & 4.4 \\
\hline 12 & CRT0806-2-37 & 330 & 16533 & 2.2 & 1.89822 & 2.2 \\
\hline 12 & CRT08062-42 & 98 & 12682 & 0.9 & 2.08233 & 3.8 \\
\hline 12 & CRT0806-2-65 & 75 & 5467 & 1.9 & 2.15586 & 7.8 \\
\hline 12 & CRT08062-25 & 61 & 7127 & 1.0 & 2.09512 & 3.7 \\
\hline 12 & CRT0806-2-53 & 148 & 2625 & 0.6 & 2.09063 & 6.2 \\
\hline 12 & CRT0806-2-3 & 328 & 14540 & 2.2 & 1.94077 & 4.3 \\
\hline 12 & CRT0806-2-51 & 165 & 9549 & 1.3 & 2.34217 & 3.8 \\
\hline 12 & CRT08062-21 & 204 & 10540 & 1.4 & 2.09504 & 5.3 \\
\hline 12 & CRT08062-18 & 314 & 36703 & 0.7 & 2.26990 & 3.1 \\
\hline 12 & CRT0806-2-49 & 373 & 24308 & 1.2 & 2.22331 & 2.5 \\
\hline 12 & CRT0806-2-41 & 403 & 33058 & 0.6 & 2.55342 & 1.9 \\
\hline 12 & CRT0806-2-24 & 489 & 4972 & 1.3 & 2.50625 & 5.1 \\
\hline 12 & CRT08062-48 & 78 & 11938 & 1.2 & 2.55252 & 5.0 \\
\hline 12 & CRT08062-47 & 131 & 19184 & 1.1 & 2.49092 & 2.5 \\
\hline 12 & CRT08062-37 & 127 & 9402 & 1.1 & 2.24880 & 5.0 \\
\hline 12 & CRT0806-2-18 & 349 & 2681 & 0.6 & 2.44283 & 5.9 \\
\hline 12 & CRT08062-55 & 408 & 3633 & 1.8 & 1.87523 & 3.9 \\
\hline 12 & CRT0806-2-7 & 197 & 2131 & 0.7 & 2.82553 & 4.3 \\
\hline 12 & CRT08062-33 & 262 & 14914 & 0.9 & 2.49794 & 3.6 \\
\hline 12 & CRT08062-41 & 199 & 27710 & 0.4 & 2.83197 & 4.3 \\
\hline 12 & CRT0806-2-20 & 590 & 35019 & 0.9 & 3.22093 & 3.1 \\
\hline 12 & CRT08062-56 & 202 & 15606 & 1.4 & 2.89600 & 4.1 \\
\hline 12 & CRT0806-2-59 & 1054 & 32688 & 0.9 & 3.17280 & 2.1 \\
\hline 12 & CRT08062-38 & 172 & 12332 & 1.0 & 2.85540 & 3.5 \\
\hline 12 & CRT08062-49 & 169 & 29414 & 1.1 & 3.30451 & 1.7 \\
\hline 12 & CRT0806-2-28 & 377 & 8694 & 0.9 & 3.43007 & 4.0 \\
\hline 12 & CRT0806-2-19 & 218 & 9370 & 1.3 & 3.12321 & 4.4 \\
\hline 12 & CRT0806-2-8 & 473 & 12367 & 1.5 & 3.24804 & 2.8 \\
\hline 12 & CRT08062-15 & 139 & 6414 & 0.9 & 2.78297 & 6.5 \\
\hline 12 & CRT08062-11 & 128 & 15912 & 0.8 & 3.49654 & 5.3 \\
\hline 12 & CRT08062-32 & 130 & 17571 & 0.5 & 3.56718 & 4.0 \\
\hline 12 & CRT08062-9 & 556 & 893 & 0.2 & 1.56337 & 10.0 \\
\hline 12 & CRT0806-2-33 & 454 & 16418 & 0.5 & 3.71582 & 4.0 \\
\hline 12 & CRT08062-62 & 150 & 2151 & 1.1 & 3.61908 & 4.9 \\
\hline 12 & CRT08062-57 & 164 & 25045 & 0.9 & 3.65638 & 2.4 \\
\hline 12 & CRT0806-2-55 & 328 & 3521 & 1.4 & 3.73318 & 5.1 \\
\hline 12 & CRT0806-2-56 & 337 & 26526 & 0.6 & 4.30251 & 2.6 \\
\hline 12 & CRT08062-60 & 204 & 24341 & 0.7 & 4.13317 & 2.3 \\
\hline 12 & CRT08062-44 & 159 & 30062 & 1.3 & 4.34512 & 2.4 \\
\hline 12 & CRT08062-19 & 534 & 61483 & 2.3 & 4.15005 & 1.9 \\
\hline 12 & CRT08062-31 & 600 & 14895 & 1.2 & 3.53373 & 6.4 \\
\hline 12 & CRT0806-2-30 & 347 & 30672 & 1.2 & 4.50261 & 4.2 \\
\hline
\end{tabular}


Kimbrough, D.L., Grove, M., Gehrels, G.E., Dorsey, R.J., Howard, K.A., Lovera, O., Aslan, A., House, P.K., and Pearthree, P.A., 2015, Detrital zircon U-Pb provenance of the Colorado River: A 5 m.y. record of incision into cover strata overlying the Colorado Plateau and adjacent regions: Geosphere, v. 11, doi:10.1130/GES00982.1.

TABLE DR7. U-Pb ZIRCON LA-ICP-MS GEOCHRONOLOGIC ANALYSES OF COLORADO RIVER BASIN \& ASSOCIATED SAMPLES.

\begin{tabular}{|c|c|c|c|c|c|c|}
\hline & & & & & & opic ra \\
\hline ID\# & Analysis & $\begin{array}{c}\text { U } \\
(\mathrm{ppm})\end{array}$ & $\begin{array}{l}206 \mathrm{~Pb} \\
204 \mathrm{~Pb}\end{array}$ & U/Th & $\begin{array}{c}207 \mathrm{~Pb}^{*} \\
235 \mathrm{U}\end{array}$ & $\begin{array}{c} \pm \\
(\%) \\
\end{array}$ \\
\hline 12 & CRT0806-2-4 & 599 & 39378 & 1.9 & 4.36312 & 2.6 \\
\hline 12 & CRT08062-43 & 168 & 30749 & 1.6 & 4.50739 & 3.8 \\
\hline 12 & CRT0806-2-54 & 302 & 31646 & 0.9 & 4.68183 & 2.5 \\
\hline 12 & CRT0806-2-12 & 535 & 15878 & 1.2 & 3.88727 & 5.6 \\
\hline 12 & CRT0806-2-39 & 1992 & 62520 & 2.5 & 4.39526 & 2.2 \\
\hline 12 & CRT0806-2-15 & 422 & 15198 & 1.0 & 4.59072 & 2.8 \\
\hline 12 & CRT08062-14 & 447 & 67167 & 1.7 & 4.75019 & 4.5 \\
\hline 12 & CRT0806-2-5 & 461 & 45323 & 1.7 & 4.62082 & 2.4 \\
\hline 12 & CRT08062-39 & 301 & 58391 & 1.0 & 4.52768 & 2.9 \\
\hline 12 & CRT0806-2-42 & 806 & 4785 & 1.1 & 3.88489 & 7.5 \\
\hline 12 & CRT0806-2-43 & 189 & 24466 & 0.9 & 4.87540 & 3.5 \\
\hline 12 & CRT0806-2-2 & 1022 & 12534 & 5.1 & 3.89341 & 7.4 \\
\hline 12 & CRT0806-2-21 & 413 & 48211 & 1.1 & 4.89438 & 2.9 \\
\hline 12 & CRT08062-50 & 58 & 10084 & 0.4 & 4.81296 & 2.1 \\
\hline 12 & CRT08062-17 & 87 & 25437 & 0.5 & 5.13553 & 2.3 \\
\hline 12 & CRT0806-2-6 & 640 & 57706 & 3.7 & 4.69075 & 1.8 \\
\hline 12 & CRT0806-2-35 & 1742 & 11743 & 1.6 & 4.44689 & 6.5 \\
\hline 12 & CRT08062-6 & 20 & 3617 & 2.3 & 5.21133 & 2.9 \\
\hline 12 & CRT08062-30 & 64 & 16084 & 1.8 & 5.67801 & 3.8 \\
\hline 12 & CRT08062-51 & 202 & 14726 & 1.1 & 5.48534 & 4.3 \\
\hline 12 & CRT08062-28 & 69 & 17800 & 0.4 & 13.04396 & 3.2 \\
\hline 12 & CRT0806-2-38 & 159 & 10476 & 1.0 & 11.16513 & 7.7 \\
\hline 12 & CRT0806-2-11 & 175 & 6833 & 1.0 & 11.43836 & 4.0 \\
\hline 12 & CRT0806-2-10 & 244 & 17344 & 0.9 & 12.46989 & 2.7 \\
\hline 12 & CRT08062-40 & 240 & 92700 & 3.0 & 15.04964 & 1.8 \\
\hline 13 & CRT08064-40 & 218 & 849 & 1.4 & 0.05547 & 18.8 \\
\hline 13 & CRT08064-42 & 1046 & 3013 & 4.4 & 0.07820 & 6.2 \\
\hline 13 & CRT08064-5 & 162 & 952 & 0.9 & 0.06297 & 23.0 \\
\hline 13 & CRT08064-60 & 271 & 1195 & 13.1 & 0.07549 & 10.6 \\
\hline 13 & CRT08064-12 & 494 & 2853 & 0.7 & 0.09009 & 7.9 \\
\hline 13 & CRT08064-55 & 799 & 2272 & 1.0 & 0.08713 & 8.4 \\
\hline 13 & CRT08064-45 & 902 & 1596 & 0.8 & 0.10287 & 25.5 \\
\hline 13 & CRT08064-32 & 608 & 1819 & 1.7 & 0.13124 & 19.4 \\
\hline 13 & CRT08064-49 & 2447 & 20322 & 0.9 & 0.17081 & 1.7 \\
\hline 13 & CRT08064-1 & 630 & 5276 & 0.8 & 0.19837 & 2.7 \\
\hline 13 & CRT08064-20 & 572 & 9623 & 1.6 & 0.20930 & 3.5 \\
\hline 13 & CRT08064-56 & 1303 & 13643 & 1.9 & 0.23998 & 3.1 \\
\hline 13 & CRT08064-26 & 212 & 2855 & 2.0 & 0.27214 & 7.6 \\
\hline 13 & CRT08064-16 & 343 & 5673 & 1.7 & 0.28101 & 3.9 \\
\hline 13 & CRT08064-52 & 741 & 5211 & 1.0 & 0.61226 & 20.1 \\
\hline 13 & CRT08064-8 & 854 & 19259 & 2.5 & 0.54370 & 2.7 \\
\hline 13 & CRT08064-31 & 221 & 6582 & 1.5 & 0.59870 & 4.1 \\
\hline 13 & CRT08064-59 & 162 & 8119 & 1.1 & 0.89025 & 3.2 \\
\hline 13 & CRT08064-18 & 125 & 5397 & 1.2 & 0.88645 & 7.3 \\
\hline 13 & CRT08064-51 & 272 & 14135 & 2.7 & 1.58660 & 4.0 \\
\hline 13 & CRT08064-29 & 154 & 10417 & 2.5 & 2.13789 & 1.7 \\
\hline 13 & CRT08064-2 & 325 & 23841 & 2.5 & 2.19177 & 1.9 \\
\hline 13 & CRT08064-48 & 95 & 7559 & 2.0 & 2.12926 & 3.5 \\
\hline 13 & CRT08064-38 & 594 & 51011 & 2.1 & 2.54702 & 1.5 \\
\hline 13 & CRT08064-15 & 55 & 6522 & 2.0 & 2.29147 & 3.5 \\
\hline 13 & CRT08064-54 & 218 & 23342 & 1.1 & 2.94560 & 2.5 \\
\hline 13 & CRT08064-11 & 327 & 38215 & 2.7 & 2.77373 & 1.8 \\
\hline 13 & CRT08064-33 & 187 & 23730 & 1.9 & 3.01537 & 2.4 \\
\hline 13 & CRT08064-43 & 362 & 28094 & 1.7 & 3.06729 & 3.8 \\
\hline 13 & CRT08064-21 & 119 & 26892 & 2.2 & 2.99660 & 2.3 \\
\hline 13 & CRT08064-7 & 229 & 20714 & 1.5 & 3.29825 & 1.6 \\
\hline 13 & CRT08064-13 & 79 & 11222 & 1.7 & 3.83605 & 2.3 \\
\hline 13 & CRT08064-47 & 109 & 11595 & 1.4 & 4.22535 & 4.0 \\
\hline 13 & CRT08064-58 & 189 & 19016 & 1.7 & 4.18224 & 1.4 \\
\hline 13 & CRT08064-46 & 78 & 8640 & 1.1 & 4.12328 & 2.2 \\
\hline 13 & CRT08064-27 & 1022 & 14221 & 3.5 & 3.29903 & 26.2 \\
\hline 13 & CRT08064-36 & 171 & 20012 & 1.2 & 4.14357 & 2.6 \\
\hline 13 & CRT08064-22 & 248 & 31251 & 1.7 & 4.34230 & 2.3 \\
\hline 13 & CRT08064-41 & 423 & 23376 & 1.1 & 4.46310 & 4.6 \\
\hline 13 & CRT08064-37 & 349 & 35855 & 2.5 & 4.53301 & 2.9 \\
\hline 13 & CRT08064-14 & 395 & 61853 & 2.1 & 4.43224 & 2.6 \\
\hline 13 & CRT08064-39 & 692 & 63910 & 1.9 & 4.46686 & 3.3 \\
\hline 13 & CRT08064-50 & 588 & 61312 & 4.4 & 4.36559 & 2.2 \\
\hline 13 & CRT08064-53 & 258 & 12504 & 1.5 & 4.60453 & 3.5 \\
\hline 13 & CRT08064-4 & 390 & 39643 & 1.1 & 4.33488 & 3.1 \\
\hline 13 & CRT08064-35 & 400 & 46801 & 1.9 & 4.38422 & 1.6 \\
\hline 13 & CRT08064-34 & 576 & 66697 & 1.7 & 4.36686 & 1.9 \\
\hline 13 & CRT08064-3 & 302 & 19662 & 2.0 & 4.36826 & 1.5 \\
\hline 13 & CRT08064-23 & 217 & 18325 & 2.2 & 4.26501 & 11.8 \\
\hline 13 & CRT08064-6 & 348 & 16068 & 1.9 & 4.14125 & 2.9 \\
\hline 13 & CRT08064-17 & 263 & 31749 & 4.6 & 3.97874 & 3.2 \\
\hline 13 & CRT08064-19 & 239 & 11092 & 0.8 & 3.67675 & 10.6 \\
\hline 13 & CRT08064-30 & 427 & 26457 & 1.0 & 4.40217 & 6.2 \\
\hline 13 & CRT08064-57 & 617 & 20979 & 2.7 & 4.70246 & 1.8 \\
\hline 13 & CRT08064-24 & 53 & 7277 & 1.1 & 4.25896 & 5.1 \\
\hline 14 & CRT08065-51 & 1033 & 4860 & 1.8 & 0.05981 & 3.2 \\
\hline 14 & CRT0806-5-48 & 817 & 5694 & 0.9 & 0.06069 & 5.2 \\
\hline 14 & CRT08065-62 & 131 & 1383 & 1.8 & 0.05744 & 26.0 \\
\hline 14 & CRT08065-45 & 279 & 2960 & 5.1 & 0.06996 & 9.6 \\
\hline 14 & CRT08065-61 & 142 & 623 & 1.0 & 0.08706 & 34.9 \\
\hline 14 & CRT0806-5-58 & 577 & 4820 & 0.7 & 0.10074 & 6.4 \\
\hline 14 & CRT0806-5-2 & 344 & 2190 & 0.6 & 0.09467 & 5.3 \\
\hline 14 & CRT08065-54 & 65 & 973 & 0.8 & 0.13383 & 18.8 \\
\hline 14 & CRT0806-5-27 & 378 & 4171 & 0.5 & 0.15252 & 4.6 \\
\hline 14 & CRT08065-47 & 114 & 2026 & 0.9 & 0.14360 & 8.8 \\
\hline
\end{tabular}


Kimbrough, D.L., Grove, M., Gehrels, G.E., Dorsey, R.J., Howard, K.A., Lovera, O., Aslan, A., House, P.K., and Pearthree, P.A., 2015, Detrital zircon U-Pb provenance of the Colorado River: A 5 m.y. record of incision into cover strata overlying the Colorado Plateau and adjacent regions: Geosphere, v. 11, doi:10.1130/GES00982.1.

TABLE DR7. U-Pb ZIRCON LA-ICP-MS GEOCHRONOLOGIC ANALYSES OF COLORADO RIVER BASIN \& ASSOCIATED SAMPLES.

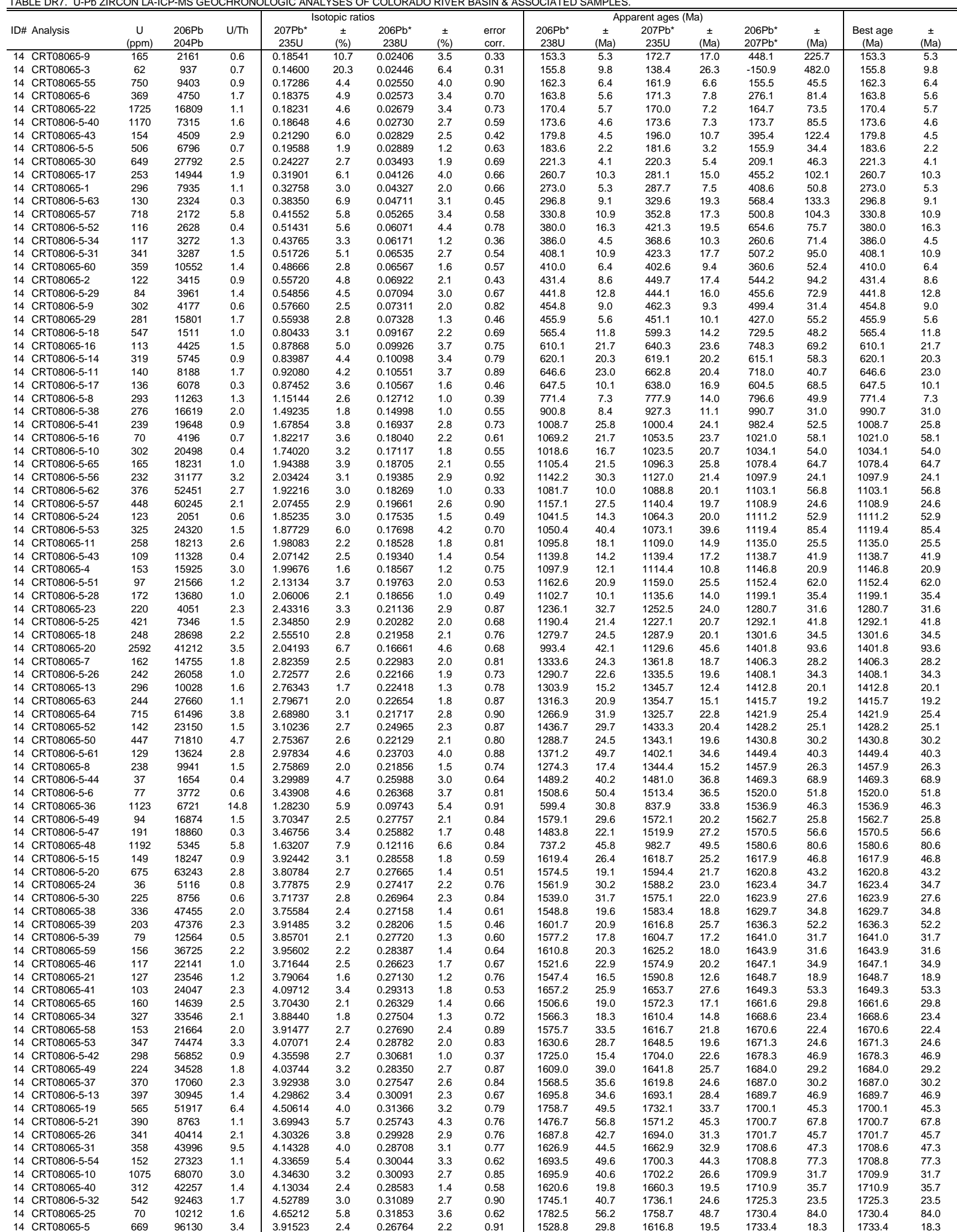


Kimbrough, D.L., Grove, M., Gehrels, G.E., Dorsey, R.J., Howard, K.A., Lovera, O., Aslan, A., House, P.K., and Pearthree, P.A., 2015, Detrital zircon U-Pb provenance of the Colorado River: A 5 m.y. record of incision into cover strata overlying the Colorado Plateau and adjacent regions: Geosphere, v. 11, doi:10.1130/GES00982.1.

TABLE DR7. U-Pb ZIRCON LA-ICP-MS GEOCHRONOLOGIC ANALYSES OF COLORADO RIVER BASIN \& ASSOCIATED SAMPLES.

\begin{tabular}{|c|c|c|c|c|c|c|}
\hline & & & & & & opic ra \\
\hline ID\# & Analysis & $\begin{array}{c}U \\
(\mathrm{ppm})\end{array}$ & $\begin{array}{l}206 \mathrm{~Pb} \\
204 \mathrm{~Pb}\end{array}$ & $\mathrm{U} / \mathrm{Th}$ & $\begin{array}{c}207 \mathrm{~Pb}^{*} \\
235 \mathrm{U}\end{array}$ & $\begin{array}{c} \pm \\
(\%)\end{array}$ \\
\hline 14 & CRT0806-5-59 & 307 & 62044 & 1.9 & 3.68554 & 5.3 \\
\hline 14 & CRT0806-5-22 & 145 & 24514 & 1.7 & 4.65282 & 2.9 \\
\hline 14 & CRT08065-56 & 588 & 53762 & 1.7 & 3.97383 & 5.4 \\
\hline 14 & CRT08065-15 & 382 & 3540 & 2.5 & 3.70498 & 7.1 \\
\hline 14 & CRT0806-5-55 & 158 & 3463 & 0.6 & 4.28746 & 3.5 \\
\hline 14 & CRT08065-28 & 313 & 33493 & 2.3 & 4.92061 & 3.5 \\
\hline 14 & CRT0806-5-7 & 63 & 10684 & 0.7 & 5.03008 & 3.2 \\
\hline 14 & CRT0806-5-45 & 77 & 21891 & 0.7 & 4.97460 & 2.9 \\
\hline 14 & CRT08065-32 & 73 & 9273 & 1.7 & 4.90119 & 2.7 \\
\hline 14 & CRT0806-5-35 & 206 & 15330 & 1.6 & 4.73681 & 3.8 \\
\hline 14 & CRT08065-12 & 108 & 13054 & 2.5 & 5.29633 & 2.1 \\
\hline 14 & CRT0806-5-3 & 447 & 2652 & 0.8 & 5.05877 & 9.7 \\
\hline 14 & CRT0806-5-50 & 478 & 125087 & 2.8 & 8.66926 & 3.5 \\
\hline 14 & CRT08065-33 & 446 & 68458 & 2.9 & 10.82569 & 2.4 \\
\hline 14 & CRT08065-44 & 192 & 90851 & 6.6 & 10.75151 & 2.0 \\
\hline 14 & CRT0806-5-23 & 60 & 26062 & 0.7 & 12.98308 & 3.3 \\
\hline 14 & CRT08065-42 & 201 & 46021 & 1.2 & 12.63702 & 1.9 \\
\hline 14 & CRT0806-5-1 & 72 & 18078 & 0.9 & 15.25566 & 2.9 \\
\hline 14 & CRT08065-35 & 41 & 12087 & 1.0 & 14.94729 & 5.6 \\
\hline 14 & CRT08065-27 & 95 & 26460 & 17.2 & 16.06009 & 4.3 \\
\hline 15 & CRTO806-6-58 & 136 & 812 & 1.3 & 0.07340 & 9.5 \\
\hline 15 & CRTO806-6-32 & 494 & 2343 & 0.7 & 0.09297 & 3.1 \\
\hline 15 & CRTO806-6-13 & 196 & 2427 & 1.6 & 0.17003 & 4.0 \\
\hline 15 & CRTO806-6-15 & 120 & 1865 & 1.0 & 0.16670 & 5.1 \\
\hline 15 & CRTO806-6-46 & 236 & 2930 & 0.9 & 0.17857 & 3.7 \\
\hline 15 & CRTO806-6-23 & 210 & 1982 & 0.5 & 0.17640 & 3.4 \\
\hline 15 & CRTO806-6-44 & 128 & 1612 & 1.9 & 0.19736 & 4.8 \\
\hline 15 & CRTO806-6-17 & 164 & 3115 & 1.1 & 0.20234 & 3.9 \\
\hline 15 & CRTO806-6-7 & 85 & 1518 & 0.5 & 0.19078 & 5.4 \\
\hline 15 & CRTO806-6-37 & 130 & 2062 & 1.0 & 0.23023 & 4.0 \\
\hline 15 & CRTO806-6-6 & 468 & 2887 & 1.4 & 0.24210 & 3.5 \\
\hline 15 & CRTO806-6-45 & 43 & 695 & 0.7 & 0.17816 & 17.6 \\
\hline 15 & CRTO806-6-59 & 349 & 4921 & 0.6 & 0.23251 & 3.2 \\
\hline 15 & CRTO806-6-48 & 160 & 2548 & 0.5 & 0.23542 & 4.2 \\
\hline 15 & CRTO806-6-65 & 193 & 3436 & 1.3 & 0.24133 & 5.2 \\
\hline 15 & CRTO806-6-1 & 73 & 1403 & 0.5 & 0.21758 & 7.5 \\
\hline 15 & CRTO806-6-11 & 178 & 2757 & 1.0 & 0.23407 & 2.0 \\
\hline 15 & CRTO806-6-26 & 190 & 3331 & 0.8 & 0.24013 & 3.2 \\
\hline 15 & CRTO806-6-3 & 231 & 4210 & 0.9 & 0.23009 & 2.4 \\
\hline 15 & CRTO806-6-22 & 1031 & 6863 & 0.7 & 0.24396 & 2.9 \\
\hline 15 & CRTO806-6-21 & 354 & 2981 & 0.8 & 0.26070 & 7.3 \\
\hline 15 & CRTO806-6-19 & 273 & 4534 & 0.7 & 0.24568 & 4.3 \\
\hline 15 & CRTO806-6-56 & 162 & 3363 & 1.3 & 0.30530 & 6.8 \\
\hline 15 & CRTO806-6-40 & 814 & 1795 & 1.3 & 0.60346 & 6.2 \\
\hline 15 & CRTO806-6-35 & 68 & 239 & 0.4 & 0.89535 & 45.6 \\
\hline 15 & CRTO806-6-8 & 143 & 1323 & 1.1 & 0.75338 & 6.8 \\
\hline 15 & CRTO806-6-24 & 101 & 4657 & 0.7 & 0.73250 & 2.2 \\
\hline 15 & CRTO806-6-31 & 132 & 8632 & 1.4 & 0.78257 & 2.0 \\
\hline 15 & CRTO806-6-47 & 239 & 11703 & 0.9 & 0.80887 & 3.1 \\
\hline 15 & CRTO806-6-12 & 189 & 10373 & 0.8 & 0.84682 & 1.4 \\
\hline 15 & CRTO806-6-50 & 422 & 24296 & 1.4 & 0.96196 & 2.1 \\
\hline 15 & CRTO806-6-29 & 231 & 5352 & 2.1 & 1.56306 & 4.2 \\
\hline 15 & CRTO806-6-57 & 98 & 6821 & 0.8 & 1.55450 & 2.0 \\
\hline 15 & CRTO806-6-53 & 105 & 7832 & 1.9 & 1.60467 & 3.2 \\
\hline 15 & CRTO806-6-38 & 708 & 42962 & 4.8 & 1.68052 & 4.0 \\
\hline 15 & CRTO806-6-62 & 82 & 4766 & 1.5 & 1.71359 & 4.3 \\
\hline 15 & CRTO806-6-9 & 249 & 25642 & 1.9 & 1.73218 & 1.6 \\
\hline 15 & CRTO806-6-42 & 170 & 1036 & 1.2 & 1.71907 & 8.6 \\
\hline 15 & CRTO806-6-54 & 234 & 24056 & 1.2 & 1.91375 & 2.2 \\
\hline 15 & CRTO806-6-39 & 117 & 9275 & 1.3 & 2.13939 & 2.6 \\
\hline 15 & CRTO806-6-25 & 94 & 7220 & 2.0 & 2.12744 & 2.0 \\
\hline 15 & CRTO806-6-52 & 248 & 21031 & 1.9 & 2.15707 & 2.8 \\
\hline 15 & CRTO806-6-61 & 49 & 4052 & 1.0 & 2.16999 & 2.2 \\
\hline 15 & CRTO806-6-36 & 594 & 21736 & 1.5 & 3.05358 & 2.1 \\
\hline 15 & CRTO806-6-14 & 514 & 67359 & 2.4 & 3.05960 & 1.6 \\
\hline 15 & CRTO806-6-43 & 149 & 15316 & 2.1 & 3.12341 & 2.6 \\
\hline 15 & CRTO806-6-55 & 164 & 16843 & 1.2 & 3.20435 & 2.4 \\
\hline 15 & CRTO806-6-18 & 67 & 6855 & 1.2 & 4.17921 & 2.6 \\
\hline 15 & CRTO806-6-51 & 238 & 23030 & 2.0 & 4.21672 & 3.0 \\
\hline 15 & CRT0806-6-28 & 871 & 72180 & 3.8 & 4.09702 & 2.1 \\
\hline 15 & CRTO806-6-34 & 149 & 10632 & 1.2 & 4.30502 & 3.0 \\
\hline 15 & CRTO806-6-16 & 76 & 6573 & 0.6 & 4.45875 & 4.0 \\
\hline 15 & CRTO806-6-2 & 158 & 18240 & 1.3 & 4.34237 & 1.4 \\
\hline 15 & CRTO806-6-27 & 152 & 23585 & 0.9 & 4.41904 & 6.0 \\
\hline 15 & CRTO806-6-60 & 375 & 25078 & 1.2 & 4.21538 & 2.9 \\
\hline 15 & CRTO806-6-10 & 308 & 47180 & 2.1 & 4.76956 & 1.9 \\
\hline 15 & CRTO806-6-30 & 56 & 19984 & 1.7 & 8.86661 & 3.7 \\
\hline 15 & CRTO806-6-20 & 97 & 28796 & 1.9 & 11.34610 & 1.7 \\
\hline 15 & CRTO806-6-4 & 45 & 9633 & 0.5 & 13.23566 & 2.3 \\
\hline 16 & CRT08067-56 & 351 & 2123 & 2.0 & 0.08170 & 9.3 \\
\hline 16 & CRT08067-25 & 1595 & 7486 & 2.8 & 0.08656 & 2.2 \\
\hline 16 & CRT08067-41 & 208 & 1540 & 1.4 & 0.09147 & 11.3 \\
\hline 16 & CRT08067-11 & 178 & 3282 & 2.0 & 0.13427 & 7.8 \\
\hline 16 & CRT08067-65 & 265 & 190 & 0.6 & 0.26214 & 27.4 \\
\hline 16 & CRT08067-37 & 179 & 2107 & 3.5 & 0.19666 & 11.7 \\
\hline 16 & CRT08067-12 & 102 & 1552 & 1.5 & 0.19014 & 12.7 \\
\hline 16 & CRT08067-15 & 322 & 2879 & 3.3 & 0.23724 & 4.9 \\
\hline 16 & CRT08067-13 & 168 & 2342 & 2.2 & 0.21808 & 7.2 \\
\hline 16 & CRT08067-34 & 432 & 6585 & 1.3 & 0.26263 & 5.4 \\
\hline 16 & CRT08067-27 & 466 & 5336 & 1.8 & 0.25113 & 6.9 \\
\hline
\end{tabular}


Kimbrough, D.L., Grove, M., Gehrels, G.E., Dorsey, R.J., Howard, K.A., Lovera, O., Aslan, A., House, P.K., and Pearthree, P.A., 2015, Detrital zircon U-Pb provenance of the Colorado River: A 5 m.y. record of incision into cover strata overlying the Colorado Plateau and adjacent regions: Geosphere, v. 11, doi:10.1130/GES00982.1.

TABLE DR7. U-Pb ZIRCON LA-ICP-MS GEOCHRONOLOGIC ANALYSES OF COLORADO RIVER BASIN \& ASSOCIATED SAMPLES.

\begin{tabular}{|c|c|c|c|c|c|c|}
\hline & & & & & & opic ra \\
\hline ID\# & Analysis & $\begin{array}{c}\text { U } \\
(\mathrm{ppm})\end{array}$ & $\begin{array}{l}206 \mathrm{~Pb} \\
204 \mathrm{~Pb}\end{array}$ & $\mathrm{U} / \mathrm{Th}$ & $\begin{array}{c}207 \mathrm{~Pb}^{*} \\
235 \mathrm{U}\end{array}$ & $\begin{array}{c} \pm \\
(\%) \\
\end{array}$ \\
\hline 16 & CRT08067-58 & 303 & 4436 & 1.9 & 0.25397 & 3.8 \\
\hline 16 & CRT08067-48 & 411 & 8204 & 1.7 & 0.26113 & 2.9 \\
\hline 16 & CRT08067-51 & 310 & 1207 & 1.3 & 0.21192 & 21.4 \\
\hline 16 & CRT08067-44 & 252 & 3754 & 1.5 & 0.26547 & 5.1 \\
\hline 16 & CRT08067-24 & 413 & 5810 & 2.7 & 0.26527 & 5.5 \\
\hline 16 & CRT08067-46 & 249 & 1897 & 1.8 & 0.27923 & 4.7 \\
\hline 16 & CRT08067-18 & 481 & 2799 & 0.6 & 0.26517 & 7.5 \\
\hline 16 & CRT08067-57 & 39 & 1609 & 1.9 & 0.37351 & 20.6 \\
\hline 16 & CRT08067-49 & 72 & 1882 & 1.5 & 0.43435 & 5.6 \\
\hline 16 & CRT08067-42 & 298 & 9375 & 1.4 & 0.46864 & 2.8 \\
\hline 16 & CRT08067-59 & 502 & 9540 & 2.3 & 0.51426 & 3.7 \\
\hline 16 & CRT08067-32 & 320 & 3562 & 0.4 & 0.76587 & 5.2 \\
\hline 16 & CRT08067-28 & 202 & 9353 & 1.0 & 0.98082 & 2.2 \\
\hline 16 & CRT08067-26 & 108 & 6683 & 3.7 & 1.53170 & 2.2 \\
\hline 16 & CRT08067-61 & 107 & 8146 & 1.6 & 1.76929 & 1.5 \\
\hline 16 & CRT08067-21 & 158 & 11332 & 2.8 & 1.72945 & 5.3 \\
\hline 16 & CRT08067-39 & 339 & 21157 & 3.4 & 1.77009 & 2.8 \\
\hline 16 & CRT08067-50 & 373 & 39536 & 4.0 & 1.82468 & 1.4 \\
\hline 16 & CRT08067-23 & 124 & 10916 & 2.0 & 1.94519 & 3.1 \\
\hline 16 & CRT08067-64 & 200 & 26726 & 4.2 & 1.91053 & 1.5 \\
\hline 16 & CRT08067-8 & 167 & 12266 & 4.1 & 1.48406 & 6.6 \\
\hline 16 & CRT08067-63 & 90 & 8533 & 3.2 & 1.93331 & 2.6 \\
\hline 16 & CRT08067-4 & 122 & 8015 & 3.3 & 1.48614 & 8.9 \\
\hline 16 & CRT08067-17 & 252 & 27643 & 1.8 & 1.95824 & 2.1 \\
\hline 16 & CRT08067-1 & 190 & 9104 & 1.4 & 2.03458 & 3.5 \\
\hline 16 & CRT08067-52 & 252 & 22399 & 1.7 & 2.08351 & 2.6 \\
\hline 16 & CRT08067-30 & 521 & 15285 & 5.6 & 1.99517 & 6.4 \\
\hline 16 & CRT08067-10 & 296 & 4264 & 1.6 & 1.89306 & 6.3 \\
\hline 16 & CRT08067-29 & 238 & 37605 & 3.4 & 2.27784 & 2.6 \\
\hline 16 & CRT08067-47 & 28 & 3023 & 3.8 & 2.14187 & 4.8 \\
\hline 16 & CRT08067-16 & 88 & 8974 & 3.3 & 2.73432 & 5.2 \\
\hline 16 & CRT08067-36 & 175 & 22820 & 2.7 & 2.99686 & 2.4 \\
\hline 16 & CRT08067-60 & 308 & 10631 & 2.3 & 2.86204 & 4.6 \\
\hline 16 & CRT08067-3 & 580 & 24129 & 3.1 & 2.97809 & 4.4 \\
\hline 16 & CRT08067-14 & 527 & 6925 & 2.7 & 3.04300 & 2.8 \\
\hline 16 & CRT08067-19 & 431 & 12024 & 2.1 & 2.81208 & 3.6 \\
\hline 16 & CRT08067-40 & 77 & 10072 & 1.6 & 3.17362 & 3.5 \\
\hline 16 & CRT08067-33 & 454 & 2713 & 2.0 & 3.04064 & 8.9 \\
\hline 16 & CRT08067-20 & 211 & 5777 & 1.5 & 3.61465 & 2.6 \\
\hline 16 & CRT08067-22 & 243 & 15398 & 2.7 & 3.36386 & 5.9 \\
\hline 16 & CRT08067-7 & 88 & 7362 & 1.1 & 3.87187 & 2.4 \\
\hline 16 & CRT08067-54 & 436 & 26194 & 3.4 & 4.11236 & 1.7 \\
\hline 16 & CRT08067-38 & 546 & 61322 & 4.7 & 4.37017 & 3.3 \\
\hline 16 & CRT08067-2 & 366 & 12734 & 2.9 & 4.06462 & 2.3 \\
\hline 16 & CRT08067-5 & 255 & 15263 & 1.1 & 4.24314 & 3.6 \\
\hline 16 & CRT08067-53 & 219 & 22912 & 2.0 & 4.35885 & 3.4 \\
\hline 16 & CRT08067-35 & 504 & 42090 & 1.5 & 4.13733 & 2.0 \\
\hline 16 & CRT08067-43 & 273 & 71407 & 2.8 & 4.75229 & 1.7 \\
\hline 16 & CRT08067-55 & 966 & 7748 & 3.0 & 4.34811 & 6.7 \\
\hline 16 & CRT08067-31 & 75 & 8810 & 0.8 & 5.29632 & 2.9 \\
\hline 16 & CRT08067-6 & 128 & 27014 & 1.5 & 6.34668 & 2.6 \\
\hline 16 & CRT08067-9 & 320 & 34168 & 3.0 & 6.74429 & 2.2 \\
\hline 16 & CRT08067-45 & 23 & 8691 & 3.4 & 13.71727 & 1.6 \\
\hline 17 & CRTO-806-8-56 & 343 & 1789 & 0.9 & 0.08977 & 5.7 \\
\hline 17 & CRTO-806-8-21 & 193 & 1897 & 1.2 & 0.15974 & 13.2 \\
\hline 17 & CRTO-806-8-22 & 136 & 1788 & 1.2 & 0.16246 & 9.5 \\
\hline 17 & CRTO-806-8-27 & 703 & 7136 & 0.8 & 0.18158 & 3.9 \\
\hline 17 & CRTO-806-8-59 & 515 & 2393 & 1.1 & 0.39683 & 11.8 \\
\hline 17 & CRTO-806-8-37 & 763 & 1045 & 4.1 & 0.41280 & 8.3 \\
\hline 17 & CRTO-806-8-43 & 451 & 9205 & 2.5 & 0.43993 & 2.5 \\
\hline 17 & CRTO-806-8-35 & 281 & 6219 & 3.1 & 0.48554 & 2.7 \\
\hline 17 & CRTO-806-8-26 & 176 & 1826 & 31.4 & 0.54574 & 6.7 \\
\hline 17 & CRTO-806-8-30 & 373 & 4420 & 0.8 & 1.45393 & 3.9 \\
\hline 17 & CRTO-806-8-33 & 556 & 44294 & 2.5 & 1.78969 & 3.1 \\
\hline 17 & CRTO-806-8-31 & 205 & 25533 & 1.8 & 1.76559 & 4.3 \\
\hline 17 & CRTO-806-8-18 & 101 & 1630 & 2.5 & 1.80868 & 7.0 \\
\hline 17 & CRTO-806-8-25 & 187 & 3119 & 0.7 & 2.00045 & 3.3 \\
\hline 17 & CRTO-806-8-60 & 195 & 18465 & 3.4 & 2.42169 & 2.8 \\
\hline 17 & CRTO-806-8-52 & 304 & 33237 & 1.5 & 2.91683 & 3.0 \\
\hline 17 & CRTO-806-8-32 & 151 & 1138 & 0.4 & 3.02279 & 2.6 \\
\hline 17 & CRTO-806-8-29 & 150 & 12730 & 1.5 & 3.07700 & 2.9 \\
\hline 17 & CRTO-806-8-23 & 38 & 3909 & 0.6 & 2.97882 & 3.7 \\
\hline 17 & CRTO-806-8-9 & 124 & 26886 & 1.3 & 3.15812 & 4.1 \\
\hline 17 & CRTO-806-8-38 & 126 & 11079 & 0.8 & 3.04275 & 2.0 \\
\hline 17 & CRTO-806-8-55 & 153 & 8049 & 0.5 & 3.00245 & 4.3 \\
\hline 17 & CRTO-806-8-36 & 169 & 16022 & 1.5 & 3.18430 & 3.3 \\
\hline 17 & CRTO-806-8-41 & 479 & 3360 & 3.1 & 2.48194 & 4.8 \\
\hline 17 & CRTO-806-8-53 & 97 & 17662 & 1.0 & 3.38484 & 3.1 \\
\hline 17 & CRTO-806-8-57 & 344 & 43809 & 1.5 & 3.50690 & 4.7 \\
\hline 17 & CRTO-806-8-48 & 226 & 30342 & 0.5 & 3.67790 & 3.7 \\
\hline 17 & CRTO-806-8-5 & 275 & 3109 & 1.6 & 3.01436 & 5.7 \\
\hline 17 & CRTO-806-8-16 & 1826 & 13343 & 0.6 & 2.23531 & 7.4 \\
\hline 17 & CRTO-806-8-44 & 427 & 6888 & 2.1 & 2.87109 & 6.0 \\
\hline 17 & CRTO-806-8-58 & 137 & 15618 & 2.2 & 3.67443 & 2.3 \\
\hline 17 & CRTO-806-8-8 & 178 & 18505 & 0.9 & 3.92372 & 2.3 \\
\hline 17 & CRTO-806-8-12 & 122 & 10995 & 1.2 & 3.92784 & 2.2 \\
\hline 17 & CRTO-806-8-17 & 135 & 13416 & 1.5 & 4.01966 & 6.4 \\
\hline 17 & CRTO-806-8-42 & 556 & 18077 & 0.6 & 3.97522 & 2.9 \\
\hline 17 & CRTO-806-8-50 & 138 & 8891 & 0.8 & 4.02085 & 2.0 \\
\hline 17 & CRTO-806-8-49 & 354 & 20717 & 2.3 & 4.13601 & 3.9 \\
\hline
\end{tabular}


Kimbrough, D.L., Grove, M., Gehrels, G.E., Dorsey, R.J., Howard, K.A., Lovera, O., Aslan, A., House, P.K., and Pearthree, P.A., 2015, Detrital zircon U-Pb provenance of the Colorado River: A 5 m.y. record of incision into cover strata overlying the Colorado Plateau and adjacent regions: Geosphere, v. 11, doi:10.1130/GES00982.1.

TABLE DR7. U-Pb ZIRCON LA-ICP-MS GEOCHRONOLOGIC ANALYSES OF COLORADO RIVER BASIN \& ASSOCIATED SAMPLES.

\begin{tabular}{|c|c|c|c|c|c|c|}
\hline & & & & & & opic ra \\
\hline ID\# & $\neq$ Analysis & $\begin{array}{c}U \\
(\mathrm{ppm})\end{array}$ & $\begin{array}{l}206 \mathrm{~Pb} \\
204 \mathrm{~Pb} \\
\end{array}$ & U/Th & $\begin{array}{c}207 \mathrm{~Pb}^{*} \\
235 \mathrm{U}\end{array}$ & $\begin{array}{c} \pm \\
(\%) \\
\end{array}$ \\
\hline 17 & CRTO-806-8-11 & 138 & 21068 & 1.3 & 3.99083 & 3.8 \\
\hline 17 & CRTO-806-8-40 & 108 & 12087 & 1.1 & 4.06491 & 4.1 \\
\hline 17 & CRTO-806-8-15 & 142 & 21691 & 0.7 & 4.27932 & 2.4 \\
\hline 17 & CRTO-806-8-14 & 214 & 47372 & 1.1 & 4.19422 & 2.9 \\
\hline 17 & CRTO-806-8-34 & 192 & 25034 & 2.8 & 4.04613 & 3.4 \\
\hline 17 & CRTO-806-8-46 & 130 & 3656 & 1.0 & 3.97146 & 1.8 \\
\hline 17 & CRTO-806-8-13 & 99 & 12547 & 1.0 & 4.00529 & 3.9 \\
\hline 17 & CRTO-806-8-51 & 128 & 2599 & 2.8 & 3.57187 & 3.1 \\
\hline 17 & CRTO-806-8-47 & 44 & 1364 & 7.2 & 4.23443 & 5.7 \\
\hline 17 & CRTO-806-8-39 & 132 & 19523 & 3.4 & 4.33053 & 2.2 \\
\hline 17 & CRTO-806-8-24 & 335 & 22084 & 30.9 & 4.31192 & 2.5 \\
\hline 17 & CRTO-806-8-7 & 51 & 12899 & 3.8 & 4.61557 & 1.5 \\
\hline 17 & CRTO-806-8-6 & 190 & 37866 & 1.6 & 4.76989 & 2.7 \\
\hline 17 & CRTO-806-8-20 & 360 & 44145 & 4.0 & 4.95066 & 1.6 \\
\hline 17 & CRTO-806-8-45 & 712 & 18743 & 6.5 & 5.16741 & 3.2 \\
\hline 18 & CRTO-806-9-57 & 391 & 620 & 1.3 & 0.02173 & 21.5 \\
\hline 18 & CRTO-806-9-9 & 243 & 523 & 1.0 & 0.02833 & 21.0 \\
\hline 18 & CRTO-806-9-24 & 557 & 2350 & 0.9 & 0.03617 & 16.5 \\
\hline 18 & CRTO-806-9-52 & 1353 & 7312 & 5.4 & 0.06744 & 5.6 \\
\hline 18 & CRTO-806-9-49 & 515 & 2017 & 1.5 & 0.06998 & 6.5 \\
\hline 18 & CRTO-806-9-38 & 144 & 1751 & 0.7 & 0.17529 & 14.0 \\
\hline 18 & CRTO-806-9-60 & 1003 & 15533 & 1.0 & 0.19964 & 2.4 \\
\hline 18 & CRTO-806-9-41 & 332 & 6315 & 2.2 & 0.24702 & 4.7 \\
\hline 18 & CRTO-806-9-36 & 323 & 7629 & 2.0 & 0.39133 & 2.1 \\
\hline 18 & CRTO-806-9-11 & 387 & 17918 & 1.6 & 0.40290 & 2.9 \\
\hline 18 & CRTO-806-9-39 & 592 & 14752 & 2.1 & 0.44231 & 3.4 \\
\hline 18 & CRTO-806-9-50 & 135 & 8638 & 2.9 & 0.56178 & 3.3 \\
\hline 18 & CRTO-806-9-14 & 94 & 5523 & 2.1 & 1.60190 & 5.5 \\
\hline 18 & CRTO-806-9-54 & 231 & 18391 & 3.3 & 1.68728 & 1.8 \\
\hline 18 & CRTO-806-9-45 & 133 & 7780 & 7.9 & 1.92059 & 2.6 \\
\hline 18 & CRTO-806-9-34 & 66 & 6986 & 3.9 & 2.12109 & 3.3 \\
\hline 18 & CRTO-806-9-53 & 250 & 21472 & 2.3 & 2.19457 & 3.2 \\
\hline 18 & CRTO-806-9-4 & 250 & 37683 & 2.8 & 2.41198 & 2.9 \\
\hline 18 & CRTO-806-9-30 & 698 & 9596 & 2.2 & 2.10516 & 6.7 \\
\hline 18 & CRTO-806-9-33 & 40 & 4802 & 1.3 & 3.03335 & 4.7 \\
\hline 18 & CRTO-806-9-56 & 203 & 35269 & 2.5 & 3.09339 & 1.9 \\
\hline 18 & CRTO-806-9-13 & 474 & 13533 & 3.0 & 2.32899 & 5.1 \\
\hline 18 & CRTO-806-9-17 & 282 & 26010 & 3.2 & 3.31536 & 4.8 \\
\hline 18 & CRTO-806-9-59 & 226 & 22966 & 1.6 & 2.89361 & 1.7 \\
\hline 18 & CRTO-806-9-16 & 61 & 9699 & 1.4 & 3.18428 & 4.0 \\
\hline 18 & CRTO-806-9-51 & 302 & 25740 & 2.4 & 3.06007 & 1.8 \\
\hline 18 & CRTO-806-9-37 & 142 & 14124 & 2.5 & 3.11049 & 4.0 \\
\hline 18 & CRTO-806-9-20 & 237 & 7527 & 1.6 & 3.36129 & 4.9 \\
\hline 18 & CRTO-806-9-6 & 659 & 84060 & 2.1 & 3.34331 & 1.6 \\
\hline 18 & CRTO-806-9-2 & 113 & 16235 & 1.2 & 3.23999 & 2.7 \\
\hline 18 & CRTO-806-9-31 & 654 & 19234 & 2.6 & 2.85802 & 10.7 \\
\hline 18 & CRTO-806-9-21 & 3214 & 345178 & 19.3 & 3.85788 & 2.4 \\
\hline 18 & CRTO-806-9-27 & 78 & 14117 & 1.4 & 4.09752 & 3.2 \\
\hline 18 & CRTO-806-9-42 & 230 & 26178 & 1.1 & 4.12380 & 2.5 \\
\hline 18 & CRTO-806-9-40 & 59 & 7231 & 1.2 & 4.18685 & 3.2 \\
\hline 18 & CRTO-806-9-12 & 241 & 23604 & 0.8 & 3.80997 & 4.6 \\
\hline 18 & CRTO-806-9-29 & 173 & 21458 & 1.3 & 4.09155 & 3.4 \\
\hline 18 & CRTO-806-9-25 & 336 & 41928 & 3.3 & 4.12351 & 5.1 \\
\hline 18 & CRTO-806-9-48 & 181 & 26924 & 1.3 & 4.16453 & 4.0 \\
\hline 18 & CRTO-806-9-7 & 251 & 19062 & 2.2 & 4.32696 & 3.9 \\
\hline 18 & CRTO-806-9-32 & 120 & 6708 & 1.3 & 3.49605 & 2.0 \\
\hline 18 & CRTO-806-9-44 & 22 & 3389 & 1.6 & 4.31395 & 2.9 \\
\hline 18 & CRTO-806-9-47 & 134 & 23011 & 1.4 & 4.15275 & 3.4 \\
\hline 18 & CRTO-806-9-10 & 128 & 17938 & 4.9 & 4.55175 & 3.4 \\
\hline 18 & CRTO-806-9-15 & 2122 & 5228 & 4.2 & 4.47356 & 6.9 \\
\hline 18 & CRTO-806-9-22 & 397 & 19613 & 2.6 & 4.23741 & 3.5 \\
\hline 18 & CRTO-806-9-26 & 369 & 77787 & 4.8 & 4.62875 & 1.9 \\
\hline 18 & CRTO-806-9-5 & 349 & 21443 & 4.0 & 4.63903 & 5.1 \\
\hline 18 & CRTO-806-9-8 & 1471 & 111039 & 8.2 & 4.82314 & 9.8 \\
\hline 18 & CRTO-806-9-58 & 834 & 78881 & 3.9 & 4.64247 & 2.8 \\
\hline 18 & CRTO-806-9-28 & 198 & 4427 & 1.2 & 4.53602 & 4.4 \\
\hline 18 & CRTO-806-9-46 & 202 & 25145 & 3.8 & 4.90715 & 6.1 \\
\hline 18 & CRTO-806-9-19 & 188 & 11938 & 1.8 & 4.55709 & 8.1 \\
\hline 18 & CRTO-806-9-55 & 567 & 977 & 0.4 & 1.99758 & 13.1 \\
\hline 18 & CRTO-806-9-3 & 205 & 26438 & 1.3 & 5.85584 & 4.2 \\
\hline 18 & CRTO-806-9-43 & 42 & 11983 & 3.9 & 14.23085 & 5.3 \\
\hline 19 & $32406-1-17$ & 311 & 412 & 0.6 & 0.00888 & 33.8 \\
\hline 19 & $32406-1-22$ & 415 & 721 & 0.4 & 0.01474 & 20.5 \\
\hline 19 & $32406-1-42$ & 234 & 464 & 0.9 & 0.02357 & 13.0 \\
\hline 19 & $32406-1--5$ & 174 & 202 & 0.5 & 0.01660 & 40.5 \\
\hline 19 & $32406-1--3$ & 118 & 991 & 0.9 & 0.08129 & 12.6 \\
\hline 19 & $32406-1-12$ & 308 & 2550 & 1.5 & 0.07611 & 8.4 \\
\hline 19 & $32406-1-53$ & 1036 & 2338 & 1.5 & 0.09973 & 2.4 \\
\hline 19 & $32406-1-28$ & 278 & 4156 & 0.8 & 0.18484 & 7.1 \\
\hline 19 & $32406-1-38$ & 1409 & 24037 & 0.8 & 0.20039 & 1.9 \\
\hline 19 & $32406-1-32$ & 772 & 41758 & 11.1 & 0.52965 & 2.0 \\
\hline 19 & $32406-1-30$ & 396 & 20231 & 7.6 & 0.99473 & 4.4 \\
\hline 19 & $32406-1-47$ & 107 & 6558 & 0.9 & 1.88743 & 1.8 \\
\hline 19 & $32406-1-37$ & 134 & 14282 & 1.5 & 2.04147 & 1.4 \\
\hline 19 & $32406-1-52$ & 636 & 49146 & 1.8 & 2.97154 & 1.4 \\
\hline 19 & $32406-1-27$ & 479 & 75640 & 1.7 & 3.08430 & 4.6 \\
\hline 19 & $32406-1-24$ & 204 & 27549 & 0.8 & 3.04272 & 1.8 \\
\hline 19 & $32406-1-19$ & 541 & 4331 & 1.0 & 2.14312 & 4.2 \\
\hline 19 & $32406-1-15$ & 48 & 7353 & 0.9 & 3.08349 & 2.3 \\
\hline 19 & $32406-1-57$ & 237 & 26725 & 0.8 & 3.11486 & 3.3 \\
\hline
\end{tabular}


Kimbrough, D.L., Grove, M., Gehrels, G.E., Dorsey, R.J., Howard, K.A., Lovera, O., Aslan, A., House, P.K., and Pearthree, P.A., 2015, Detrital zircon U-Pb provenance of the Colorado River: A 5 m.y. record of incision into cover strata overlying the Colorado Plateau and adjacent regions: Geosphere, v. 11, doi:10.1130/GES00982.1.

TABLE DR7. U-Pb ZIRCON LA-ICP-MS GEOCHRONOLOGIC ANALYSES OF COLORADO RIVER BASIN \& ASSOCIATED SAMPLES.

\begin{tabular}{|c|c|c|c|c|c|c|}
\hline & & & & & & opic ra \\
\hline ID\# & * Analysis & $\begin{array}{c}U \\
(\mathrm{ppm})\end{array}$ & $\begin{array}{l}206 \mathrm{~Pb} \\
204 \mathrm{~Pb} \\
\end{array}$ & $\mathrm{U} / \mathrm{Th}$ & $\begin{array}{c}207 \mathrm{~Pb}^{*} \\
235 \mathrm{U}\end{array}$ & $\begin{array}{c} \pm \\
(\%) \\
\end{array}$ \\
\hline & $32406-1-18$ & 220 & 12233 & 0.9 & 3.09200 & 2.4 \\
\hline 19 & $32406-1-21$ & 55 & 6363 & 0.7 & 3.13914 & 4.0 \\
\hline 19 & $32406-1-26$ & 234 & 5005 & 0.7 & 3.22356 & 4.3 \\
\hline 19 & $32406-1-45$ & 135 & 8830 & 0.8 & 2.71780 & 7.1 \\
\hline 19 & $32406-1-10$ & 546 & 6503 & 1.1 & 2.70684 & 3.5 \\
\hline 19 & $32406-1-54$ & 109 & 17742 & 0.9 & 3.23461 & 1.4 \\
\hline 19 & $32406-1-23$ & 313 & 38061 & 2.7 & 3.12049 & 1.5 \\
\hline 19 & $32406-1-20$ & 216 & 36726 & 1.8 & 3.22972 & 2.5 \\
\hline 19 & $32406-1-35$ & 347 & 2972 & 1.6 & 3.29441 & 3.6 \\
\hline 19 & $32406-1-29$ & 153 & 23748 & 0.9 & 4.13174 & 7.3 \\
\hline 19 & $32406-1-31$ & 384 & 26677 & 1.2 & 4.10058 & 2.8 \\
\hline 19 & $32406-1-13$ & 160 & 4542 & 0.8 & 4.33101 & 4.6 \\
\hline 19 & $32406-1-4$ & 701 & 13360 & 0.4 & 3.66893 & 7.0 \\
\hline 19 & $32406-1-58$ & 860 & 79520 & 2.0 & 4.40770 & 2.9 \\
\hline 19 & $32406-1-59$ & 108 & 17015 & 0.7 & 4.20411 & 3.3 \\
\hline 19 & $32406-1-51$ & 144 & 41498 & 1.0 & 4.27648 & 1.9 \\
\hline 19 & $32406-1-44$ & 118 & 14761 & 1.5 & 4.27445 & 3.0 \\
\hline 19 & $32406-1-7$ & 237 & 6195 & 0.8 & 3.24258 & 4.5 \\
\hline 19 & $32406-1-48$ & 275 & 38615 & 1.6 & 4.61299 & 3.2 \\
\hline 19 & $32406-1-39$ & 383 & 2788 & 0.3 & 4.28727 & 3.1 \\
\hline 19 & $32406-1-6$ & 183 & 2344 & 0.5 & 4.08887 & 5.1 \\
\hline 19 & $32406-1-55$ & 304 & 14705 & 1.3 & 4.31614 & 1.6 \\
\hline 19 & $32406-1-46$ & 317 & 19452 & 3.3 & 4.50433 & 1.7 \\
\hline 19 & $32406-1-56$ & 272 & 34601 & 1.4 & 4.24541 & 2.2 \\
\hline 19 & $32406-1--1$ & 410 & 58123 & 2.9 & 4.73430 & 1.6 \\
\hline 19 & $32406-1-25$ & 144 & 25340 & 4.8 & 4.40202 & 3.4 \\
\hline 19 & $32406-1--2$ & 318 & 7990 & 0.8 & 5.37103 & 4.4 \\
\hline 19 & $32406-1-9$ & 112 & 57516 & 0.7 & 13.56312 & 2.3 \\
\hline 20 & CRT080610-65 & 437 & 559 & 1.0 & 0.00758 & 45.9 \\
\hline 20 & CRT080610-43 & 540 & 842 & 0.9 & 0.01991 & 13.7 \\
\hline 20 & CRT080610-11 & 139 & 1041 & 0.6 & 0.08357 & 12.5 \\
\hline 20 & CRT080610-17 & 133 & 1130 & 1.1 & 0.08305 & 15.2 \\
\hline 20 & CRT080610-32 & 171 & 1350 & 0.9 & 0.12403 & 25.2 \\
\hline 20 & CRT080610-30 & 472 & 2581 & 0.5 & 0.16667 & 7.7 \\
\hline 20 & CRT080610-68 & 559 & 7463 & 1.5 & 0.19030 & 3.0 \\
\hline 20 & CRT080610-62 & 606 & 7699 & 2.1 & 0.19915 & 3.6 \\
\hline 20 & CRT080610-61 & 64 & 1774 & 0.8 & 0.23332 & 19.6 \\
\hline 20 & CRT080610-9 & 730 & 14363 & 3.1 & 0.26046 & 3.2 \\
\hline 20 & CRT080610-20 & 19 & 631 & 4.0 & 0.20807 & 41.0 \\
\hline 20 & CRT080610-51 & 491 & 14506 & 2.5 & 0.39145 & 2.5 \\
\hline 20 & CRT080610-6 & 460 & 18855 & 1.7 & 0.40636 & 2.6 \\
\hline 20 & CRT080610-39 & 300 & 5677 & 1.4 & 0.49948 & 3.3 \\
\hline 20 & CRT080610-70 & 715 & 8504 & 16.2 & 0.48738 & 4.1 \\
\hline 20 & CRT080610-23 & 286 & 7822 & 6.4 & 0.57590 & 5.7 \\
\hline 20 & CRT080610-5 & 205 & 7861 & 1.5 & 0.57072 & 3.7 \\
\hline 20 & CRT080610-69 & 584 & 9694 & 1.0 & 0.53791 & 2.3 \\
\hline 20 & CRT080610-67 & 644 & 12275 & 1.2 & 0.55554 & 2.3 \\
\hline 20 & CRT080610-27 & 106 & 3469 & 0.9 & 0.58584 & 4.2 \\
\hline 20 & CRT080610-73 & 291 & 11829 & 1.4 & 0.61233 & 3.4 \\
\hline 20 & CRT080610-13 & 214 & 8936 & 3.4 & 0.72900 & 2.6 \\
\hline 20 & CRT080610-21 & 267 & 9767 & 2.4 & 0.80929 & 4.1 \\
\hline 20 & CRT080610-59 & 276 & 24024 & 1.9 & 0.88932 & 3.8 \\
\hline 20 & CRT080610-53 & 604 & 42940 & 2.6 & 1.71626 & 2.9 \\
\hline 20 & CRT080610-57 & 219 & 36473 & 2.4 & 1.83368 & 2.0 \\
\hline 20 & CRT080610-63 & 148 & 12998 & 1.8 & 1.85196 & 3.3 \\
\hline 20 & CRT080610-29 & 570 & 33618 & 9.1 & 1.81243 & 1.9 \\
\hline 20 & CRT080610-71 & 914 & 29339 & 30.4 & 1.69561 & 3.3 \\
\hline 20 & CRT080610-14 & 823 & 55152 & 2.4 & 1.82464 & 3.5 \\
\hline 20 & CRT080610-58 & 210 & 33109 & 1.5 & 1.93712 & 2.2 \\
\hline 20 & CRT080610-31 & 132 & 9573 & 2.2 & 1.85742 & 3.2 \\
\hline 20 & CRT080610-10 & 285 & 20896 & 2.9 & 2.05085 & 4.9 \\
\hline 20 & CRT080610-44 & 612 & 3674 & 1.0 & 1.66775 & 7.6 \\
\hline 20 & CRT080610-35 & 234 & 21278 & 2.3 & 2.17947 & 1.9 \\
\hline 20 & CRT080610-37 & 67 & 5027 & 3.2 & 1.93938 & 3.4 \\
\hline 20 & CRT080610-18 & 87 & 7406 & 1.9 & 2.03466 & 3.9 \\
\hline 20 & CRT080610-45 & 198 & 14524 & 2.9 & 2.12761 & 4.6 \\
\hline 20 & CRT080610-24 & 307 & 20727 & 3.2 & 2.42168 & 3.5 \\
\hline 20 & CRT080610-12 & 34 & 4140 & 1.5 & 2.03128 & 4.3 \\
\hline 20 & CRT080610-3 & 268 & 29820 & 3.2 & 2.58384 & 6.1 \\
\hline 20 & CRT080610-28 & 105 & 10628 & 1.5 & 2.63207 & 3.1 \\
\hline 20 & CRT080610-15 & 379 & 40161 & 2.2 & 2.41567 & 3.4 \\
\hline 20 & CRT080610-74 & 44 & 4972 & 1.5 & 2.23236 & 5.0 \\
\hline 20 & CRT080610-66 & 487 & 32925 & 1.8 & 2.59353 & 1.6 \\
\hline 20 & CRT080610-1 & 271 & 37969 & 2.5 & 2.89346 & 3.1 \\
\hline 20 & CRT080610-16 & 66 & 2415 & 1.3 & 2.31311 & 6.9 \\
\hline 20 & CRT080610-72 & 315 & 49679 & 1.7 & 2.97523 & 2.7 \\
\hline 20 & CRT080610-7 & 378 & 8547 & 2.0 & 3.15948 & 4.5 \\
\hline 20 & CRT080610-47 & 136 & 20723 & 2.0 & 3.32876 & 2.4 \\
\hline 20 & CRT080610-75 & 85 & 12563 & 1.0 & 4.10663 & 1.9 \\
\hline 20 & CRT080610-25 & 273 & 33817 & 1.8 & 4.18267 & 2.9 \\
\hline 20 & CRT080610-8 & 530 & 110615 & 2.3 & 4.40266 & 3.8 \\
\hline 20 & CRT080610-56 & 262 & 36683 & 1.6 & 4.69808 & 1.5 \\
\hline 20 & CRT080610-41 & 178 & 26799 & 2.9 & 4.77445 & 3.3 \\
\hline 20 & CRT080610-2 & 53 & 6904 & 1.0 & 4.92585 & 3.5 \\
\hline 20 & CRT080610-34 & 328 & 58453 & 2.6 & 4.78334 & 2.0 \\
\hline 20 & CRT080610-26 & 192 & 27975 & 2.3 & 5.12121 & 2.6 \\
\hline 20 & CRT080610-42 & 259 & 22481 & 1.3 & 4.99716 & 3.8 \\
\hline 20 & CRT080610-64 & 30 & 896 & 0.5 & 4.93808 & 4.8 \\
\hline 20 & CRT080610-46 & 94 & 20729 & 1.9 & 14.03937 & 2.3 \\
\hline 20 & CRT080610-4 & 122 & 34505 & 1.3 & 13.57395 & 2.5 \\
\hline
\end{tabular}


Kimbrough, D.L., Grove, M., Gehrels, G.E., Dorsey, R.J., Howard, K.A., Lovera, O., Aslan, A., House, P.K., and Pearthree, P.A., 2015, Detrital zircon U-Pb provenance of the Colorado River: A 5 m.y. record of incision into cover strata overlying the Colorado Plateau and adjacent regions: Geosphere, v. 11, doi:10.1130/GES00982.1.

TABLE DR7. U-Pb ZIRCON LA-ICP-MS GEOCHRONOLOGIC ANALYSES OF COLORADO RIVER BASIN \& ASSOCIATED SAMPLES.

\begin{tabular}{|c|c|c|c|c|c|c|}
\hline & & & & & & topic ra \\
\hline ID\# & Analysis & $\begin{array}{c}U \\
(\mathrm{ppm})\end{array}$ & $\begin{array}{l}206 \mathrm{~Pb} \\
204 \mathrm{~Pb}\end{array}$ & $\mathrm{U} / \mathrm{Th}$ & $\begin{array}{c}207 \mathrm{~Pb}^{*} \\
235 \mathrm{U}\end{array}$ & $\begin{array}{c} \pm \\
(\%) \\
\end{array}$ \\
\hline 20 & CRT080610-52 & 209 & 18727 & 2.2 & 13.28200 & 6.6 \\
\hline 20 & CRT080610-40 & 54 & 15892 & 2.8 & 16.67441 & 2.1 \\
\hline 20 & CRT080610-19 & 134 & 36055 & 1.7 & 22.61663 & 2.6 \\
\hline 21 & CRT080612-4 & 366 & 681 & 23.9 & 0.01799 & 27.4 \\
\hline 21 & CRT080612-23 & 758 & 2475 & 1.3 & 0.06539 & 6.1 \\
\hline 21 & CRT080612-64 & 506 & 3610 & 1.8 & 0.09350 & 4.8 \\
\hline 21 & CRT080612-37 & 349 & 3406 & 1.1 & 0.17195 & 4.4 \\
\hline 21 & CRT080612-42 & 574 & 3945 & 0.8 & 0.18429 & 3.4 \\
\hline 21 & CRT080612-3 & 402 & 4291 & 1.4 & 0.18252 & 4.3 \\
\hline 21 & CRT080612-43 & 541 & 7402 & 1.1 & 0.19542 & 3.4 \\
\hline 21 & CRT080612-39 & 738 & 5135 & 0.7 & 0.20722 & 2.2 \\
\hline 21 & CRT080612-30 & 723 & 8745 & 3.7 & 0.21624 & 2.5 \\
\hline 21 & CRT080612-18 & 231 & 3370 & 2.4 & 0.23948 & 4.6 \\
\hline 21 & CRT080612-46 & 97 & 1480 & 1.5 & 0.19472 & 12.1 \\
\hline 21 & CRT080612-35 & 334 & 4524 & 1.1 & 0.24415 & 4.2 \\
\hline 21 & CRT080612-51 & 479 & 6937 & 1.2 & 0.25175 & 3.0 \\
\hline 21 & CRT080612-27 & 383 & 6089 & 2.1 & 0.25015 & 2.6 \\
\hline 21 & CRT080612-55 & 230 & 1815 & 0.7 & 0.26617 & 15.9 \\
\hline 21 & CRT080612-14 & 462 & 7881 & 1.8 & 0.26077 & 2.9 \\
\hline 21 & CRT080612-44 & 668 & 11176 & 2.6 & 0.24573 & 2.3 \\
\hline 21 & CRT080612-32 & 352 & 9101 & 1.4 & 0.27692 & 3.0 \\
\hline 21 & CRT080612-29 & 1000 & 8384 & 1.5 & 0.26532 & 7.1 \\
\hline 21 & CRT080612-66 & 600 & 8753 & 0.7 & 0.25250 & 2.4 \\
\hline 21 & CRT080612-9 & 1753 & 4235 & 1.1 & 0.27910 & 2.7 \\
\hline 21 & CRT080612-59 & 211 & 4175 & 2.3 & 0.28023 & 6.3 \\
\hline 21 & CRT080612-34 & 360 & 4295 & 1.2 & 0.29838 & 3.4 \\
\hline 21 & CRT080612-40 & 891 & 1447 & 1.6 & 0.31135 & 5.0 \\
\hline 21 & CRT080612-11 & 820 & 19069 & 1.8 & 0.29178 & 2.4 \\
\hline 21 & CRT080612-54 & 207 & 5271 & 2.0 & 0.46785 & 2.3 \\
\hline 21 & CRT080612-19 & 447 & 13041 & 1.7 & 0.45362 & 3.2 \\
\hline 21 & CRT080612-31 & 242 & 2427 & 2.9 & 0.59351 & 7.1 \\
\hline 21 & CRT080612-20 & 193 & 6596 & 1.4 & 0.83230 & 3.5 \\
\hline 21 & CRT080612-47 & 1264 & 13379 & 3.1 & 1.39113 & 4.9 \\
\hline 21 & CRT080612-69 & 91 & 6497 & 1.5 & 1.54273 & 3.0 \\
\hline 21 & CRT080612-7 & 193 & 14518 & 2.4 & 1.66700 & 2.8 \\
\hline 21 & CRT080612-38 & 111 & 9984 & 2.2 & 1.80485 & 4.0 \\
\hline 21 & CRT080612-60 & 305 & 43847 & 2.9 & 1.77282 & 1.7 \\
\hline 21 & CRT080612-22 & 450 & 32548 & 4.1 & 1.92197 & 2.8 \\
\hline 21 & CRT080612-67 & 126 & 7633 & 2.2 & 1.91092 & 2.5 \\
\hline 21 & CRT080612-57 & 71 & 4952 & 0.9 & 1.80318 & 4.2 \\
\hline 21 & CRT080612-36 & 491 & 41972 & 4.1 & 2.15244 & 1.8 \\
\hline 21 & CRT080612-33 & 90 & 2096 & 1.8 & 2.07105 & 4.1 \\
\hline 21 & CRT080612-56 & 245 & 24277 & 2.4 & 2.21271 & 2.8 \\
\hline 21 & CRT080612-45 & 500 & 34372 & 1.0 & 2.72696 & 2.0 \\
\hline 21 & CRT080612-50 & 93 & 6010 & 1.1 & 2.89581 & 2.7 \\
\hline 21 & CRT080612-49 & 333 & 32717 & 3.1 & 3.01028 & 2.9 \\
\hline 21 & CRT080612-65 & 89 & 7572 & 0.8 & 2.92573 & 2.8 \\
\hline 21 & CRT080612-16 & 1114 & 80540 & 4.0 & 3.06583 & 2.3 \\
\hline 21 & CRT080612-17 & 240 & 31014 & 2.9 & 3.05359 & 3.2 \\
\hline 21 & CRT080612-6 & 195 & 23597 & 1.4 & 3.41345 & 2.4 \\
\hline 21 & CRT080612-63 & 151 & 18859 & 3.0 & 3.31202 & 2.4 \\
\hline 21 & CRT080612-48 & 259 & 30533 & 1.7 & 3.79333 & 2.2 \\
\hline 21 & CRT080612-25 & 120 & 18799 & 1.6 & 3.95977 & 2.9 \\
\hline 21 & CRT080612-8 & 241 & 38896 & 2.5 & 4.02752 & 2.0 \\
\hline 21 & CRT080612-41 & 123 & 12766 & 1.5 & 4.09479 & 1.9 \\
\hline 21 & CRT080612-61 & 90 & 6642 & 1.4 & 4.04691 & 1.9 \\
\hline 21 & CRT080612-26 & 196 & 21980 & 2.3 & 4.34325 & 3.1 \\
\hline 21 & CRT080612-21 & 185 & 18994 & 3.1 & 4.14539 & 2.6 \\
\hline 21 & CRT080612-10 & 144 & 8532 & 1.0 & 4.36453 & 2.6 \\
\hline 21 & CRT080612-5 & 696 & 72990 & 4.0 & 3.29526 & 3.7 \\
\hline 21 & CRT080612-13 & 601 & 10104 & 3.9 & 3.61829 & 7.9 \\
\hline 21 & CRT080612-1 & 212 & 34189 & 1.9 & 4.46134 & 3.2 \\
\hline 21 & CRT080612-62 & 353 & 38152 & 2.0 & 4.70650 & 2.3 \\
\hline 21 & CRT080612-24 & 671 & 67693 & 3.1 & 4.48499 & 1.9 \\
\hline 21 & CRT080612-2 & 190 & 20977 & 1.5 & 5.28469 & 3.1 \\
\hline 21 & CRT080612-68 & 467 & 50398 & 2.6 & 5.63051 & 2.8 \\
\hline 21 & CRT080612-12 & 169 & 37372 & 2.2 & 13.77963 & 3.4 \\
\hline 21 & CRT080612-52 & 933 & 53925 & 1.6 & 13.31215 & 2.1 \\
\hline 22 & CRT080613-9 & 2752 & 3047 & 2.6 & 0.01761 & 7.0 \\
\hline 22 & CRT080613-11 & 835 & 1616 & 1.9 & 0.03408 & 7.2 \\
\hline 22 & CRT080613-36 & 873 & 5134 & 2.5 & 0.03497 & 5.5 \\
\hline 22 & CRT080613-3 & 156 & 827 & 2.8 & 0.11765 & 17.1 \\
\hline 22 & CRT080613-60 & 402 & 5154 & 1.0 & 0.10468 & 4.8 \\
\hline 22 & CRT080613-48 & 427 & 6050 & 2.1 & 0.12306 & 3.9 \\
\hline 22 & CRT080613-13 & 289 & 4133 & 0.7 & 0.20834 & 7.5 \\
\hline 22 & CRT080613-1 & 602 & 8670 & 1.4 & 0.19724 & 3.7 \\
\hline 22 & CRT080613-50 & 103 & 1885 & 0.6 & 0.19771 & 10.9 \\
\hline 22 & CRT080613-35 & 451 & 6960 & 0.9 & 0.20730 & 4.2 \\
\hline 22 & CRT080613-40 & 606 & 793 & 1.0 & 0.33730 & 12.2 \\
\hline 22 & CRT080613-28 & 190 & 4935 & 1.4 & 0.26648 & 3.7 \\
\hline 22 & CRT080613-20 & 97 & 3626 & 0.8 & 0.29151 & 6.2 \\
\hline 22 & CRT080613-54 & 192 & 2868 & 1.4 & 0.32421 & 9.8 \\
\hline 22 & CRT080613-14 & 353 & 8276 & 1.1 & 0.31276 & 4.6 \\
\hline 22 & CRT080613-17 & 239 & 7685 & 2.0 & 0.35104 & 3.6 \\
\hline 22 & CRT080613-32 & 150 & 7957 & 1.2 & 0.50739 & 10.4 \\
\hline 22 & CRT080613-51 & 565 & 15023 & 4.2 & 0.54139 & 2.3 \\
\hline 22 & CRT080613-5 & 154 & 2355 & 1.2 & 0.77355 & 5.1 \\
\hline 22 & CRT080613-12 & 461 & 22336 & 10.7 & 0.67483 & 1.5 \\
\hline 22 & CRT080613-34 & 248 & 18779 & 1.3 & 0.86454 & 3.6 \\
\hline 22 & CRT080613-18 & 135 & 20214 & 1.9 & 1.68297 & 2.0 \\
\hline
\end{tabular}


Kimbrough, D.L., Grove, M., Gehrels, G.E., Dorsey, R.J., Howard, K.A., Lovera, O., Aslan, A., House, P.K., and Pearthree, P.A., 2015, Detrital zircon U-Pb provenance of the Colorado River: A 5 m.y. record of incision into cover strata overlying the Colorado Plateau and adjacent regions: Geosphere, v. 11, doi:10.1130/GES00982.1.

TABLE DR7. U-Pb ZIRCON LA-ICP-MS GEOCHRONOLOGIC ANALYSES OF COLORADO RIVER BASIN \& ASSOCIATED SAMPLES.

\begin{tabular}{|c|c|c|c|c|c|c|}
\hline & & & & & & opic ra \\
\hline ID\# & Analysis & $\begin{array}{c}U \\
\text { (ppm) }\end{array}$ & $\begin{array}{l}206 \mathrm{~Pb} \\
204 \mathrm{~Pb} \\
\end{array}$ & U/Th & $\begin{array}{c}207 \mathrm{~Pb}^{*} \\
235 \mathrm{U}\end{array}$ & $\begin{array}{c} \pm \\
(\%)\end{array}$ \\
\hline 22 & CRT080613-37 & 344 & 26537 & 0.4 & 1.71590 & 1.9 \\
\hline 22 & CRT080613-27 & 144 & 19423 & 3.9 & 1.74168 & 3.0 \\
\hline 22 & CRT080613-6 & 231 & 17496 & 0.7 & 1.75504 & 2.9 \\
\hline 22 & CRT080613-2 & 92 & 9437 & 1.8 & 1.90384 & 2.8 \\
\hline 22 & CRT080613-33 & 350 & 35963 & 2.0 & 2.00135 & 2.4 \\
\hline 22 & CRT080613-8 & 64 & 8704 & 1.3 & 2.13997 & 6.1 \\
\hline 22 & CRT080613-15 & 434 & 45170 & 2.5 & 2.18530 & 1.4 \\
\hline 22 & CRT080613-58 & 124 & 23789 & 1.8 & 2.42450 & 2.2 \\
\hline 22 & CRT080613-16 & 28 & 3201 & 1.3 & 2.26830 & 3.6 \\
\hline 22 & CRT080613-38 & 31 & 7270 & 1.8 & 2.17622 & 7.4 \\
\hline 22 & CRT080613-56 & 204 & 35047 & 4.3 & 2.99745 & 2.0 \\
\hline 22 & CRT080613-47 & 775 & 61857 & 2.9 & 3.09266 & 3.1 \\
\hline 22 & CRT080613-31 & 154 & 15587 & 0.9 & 2.97584 & 3.0 \\
\hline 22 & CRT080613-10 & 112 & 19564 & 3.1 & 3.37059 & 2.6 \\
\hline 22 & CRT080613-55 & 229 & 17557 & 2.3 & 3.81038 & 7.4 \\
\hline 22 & CRT080613-49 & 379 & 100600 & 2.8 & 3.90909 & 2.7 \\
\hline 22 & CRT080613-39 & 231 & 34789 & 2.1 & 4.22128 & 2.4 \\
\hline 22 & CRT080613-29 & 197 & 28029 & 1.0 & 4.12603 & 2.0 \\
\hline 22 & CRT080613-44 & 124 & 31840 & 1.9 & 4.39702 & 2.3 \\
\hline 22 & CRT080613-4 & 99 & 14268 & 1.2 & 4.46881 & 2.0 \\
\hline 22 & CRT080613-53 & 240 & 18080 & 1.3 & 4.33663 & 3.3 \\
\hline 22 & CRT080613-23 & 231 & 7883 & 1.4 & 4.32355 & 5.2 \\
\hline 22 & CRT080613-46 & 183 & 31346 & 2.6 & 4.26801 & 5.8 \\
\hline 22 & CRT080613-19 & 305 & 52701 & 4.9 & 4.40964 & 2.6 \\
\hline 22 & CRT080613-43 & 167 & 34057 & 0.9 & 4.64039 & 2.6 \\
\hline 22 & CRT080613-42 & 109 & 22894 & 1.8 & 4.59363 & 1.7 \\
\hline 22 & CRT080613-22 & 501 & 21056 & 0.6 & 3.35817 & 6.3 \\
\hline 22 & CRT080613-57 & 571 & 122018 & 3.0 & 4.74709 & 1.8 \\
\hline 22 & CRT080613-41 & 370 & 10625 & 2.4 & 3.97582 & 6.7 \\
\hline 22 & CRT080613-45 & 575 & 80132 & 1.0 & 4.43825 & 1.7 \\
\hline 22 & CRT080613-52 & 34 & 6087 & 1.9 & 4.28242 & 2.3 \\
\hline 22 & CRT080613-24 & 356 & 5532 & 0.7 & 3.65981 & 6.3 \\
\hline 23 & $32706-3-38$ & 1730 & 3058 & 2.2 & 0.02735 & 5.7 \\
\hline 23 & 32706-3-54 & 249 & 644 & 1.4 & 0.03601 & 12.3 \\
\hline 23 & 32706-3-12 & 346 & 3540 & 1.5 & 0.08794 & 7.5 \\
\hline 23 & $32706-3-34$ & 574 & 4387 & 4.1 & 0.10670 & 3.8 \\
\hline 23 & 32706-3-35 & 922 & 6832 & 2.3 & 0.09902 & 2.5 \\
\hline 23 & $32706-3-46$ & 522 & 3782 & 1.3 & 0.11960 & 5.2 \\
\hline 23 & $32706-3-7$ & 95 & 2100 & 0.7 & 0.16200 & 11.3 \\
\hline 23 & $32706-3-3$ & 624 & 7662 & 0.9 & 0.17313 & 2.1 \\
\hline 23 & $32706-3-8$ & 413 & 8830 & 1.3 & 0.18903 & 2.4 \\
\hline 23 & 32706-3-25 & 69 & 1411 & 1.5 & 0.21747 & 6.9 \\
\hline 23 & $32706-3-4$ & 580 & 1203 & 1.0 & 0.23406 & 5.5 \\
\hline 23 & 32706-3-62 & 971 & 4210 & 1.4 & 0.21106 & 5.4 \\
\hline 23 & 32706-3-65 & 535 & 6757 & 2.8 & 0.19777 & 10.0 \\
\hline 23 & 32706-3-51 & 288 & 6730 & 3.9 & 0.22903 & 2.7 \\
\hline 23 & 32706-3-41 & 154 & 14870 & 1.1 & 1.75494 & 2.4 \\
\hline 23 & $32706-3-26$ & 128 & 18217 & 1.1 & 3.00224 & 4.9 \\
\hline 23 & $32706-3-63$ & 224 & 30904 & 1.5 & 2.74734 & 1.9 \\
\hline 23 & $32706-3-47$ & 305 & 46807 & 2.0 & 2.85281 & 2.8 \\
\hline 23 & 32706-3-56 & 220 & 41511 & 2.2 & 2.81698 & 4.4 \\
\hline 23 & $32706-3-16$ & 145 & 17828 & 1.5 & 2.79312 & 3.7 \\
\hline 23 & $32706-3-19$ & 165 & 29142 & 1.7 & 2.85560 & 2.1 \\
\hline 23 & 32706-3-33 & 157 & 17663 & 0.8 & 2.91036 & 2.4 \\
\hline 23 & $32706-3-45$ & 196 & 29800 & 1.2 & 2.73259 & 1.9 \\
\hline 23 & 32706-3-58 & 363 & 49430 & 2.1 & 2.89731 & 1.9 \\
\hline 23 & $32706-3-14$ & 386 & 53521 & 1.5 & 3.04391 & 1.4 \\
\hline 23 & 32706-3-64 & 845 & 76921 & 1.3 & 2.79212 & 3.0 \\
\hline 23 & 32706-3-37 & 609 & 84139 & 1.4 & 2.99182 & 1.5 \\
\hline 23 & $32706-3-18$ & 82 & 19448 & 1.4 & 3.08487 & 2.1 \\
\hline 23 & $32706-3-1$ & 46 & 9284 & 1.0 & 2.93228 & 2.2 \\
\hline 23 & 32706-3-30 & 2624 & 166368 & 3.0 & 2.79018 & 4.7 \\
\hline 23 & 32706-3-31 & 75 & 9653 & 1.0 & 2.95891 & 3.4 \\
\hline 23 & $32706-3-23$ & 1566 & 73713 & 4.5 & 2.91055 & 2.0 \\
\hline 23 & $32706-3-60$ & 142 & 21057 & 1.1 & 3.04313 & 4.4 \\
\hline 23 & 32706-3-32 & 54 & 5744 & 0.6 & 2.91640 & 5.2 \\
\hline 23 & 32706-3-44 & 2024 & 13118 & 1.7 & 3.04184 & 5.1 \\
\hline 23 & $32706-3-22$ & 308 & 23800 & 1.2 & 3.06023 & 4.0 \\
\hline 23 & $32706-3-43$ & 147 & 25138 & 1.3 & 3.33754 & 2.2 \\
\hline 23 & $32706-3-48$ & 74 & 5623 & 0.6 & 2.87735 & 8.7 \\
\hline 23 & 32706-3-61 & 259 & 49494 & 5.5 & 3.40722 & 1.8 \\
\hline 23 & $32706-3-13$ & 24 & 4593 & 0.7 & 3.12460 & 2.6 \\
\hline 23 & $32706-3-29$ & 22 & 3779 & 1.0 & 3.20362 & 3.3 \\
\hline 23 & $32706-3-50$ & 19 & 2220 & 1.5 & 3.31213 & 3.9 \\
\hline 23 & $32706-3-27$ & 12 & 2021 & 1.2 & 3.20924 & 6.3 \\
\hline 23 & 32706-3-49 & 295 & 46854 & 1.8 & 4.34477 & 1.7 \\
\hline 23 & $32706-3-11$ & 142 & 31484 & 0.7 & 4.27196 & 1.5 \\
\hline 23 & $32706-3-2$ & 169 & 29442 & 2.1 & 4.33947 & 1.9 \\
\hline 23 & $32706-3-28$ & 127 & 26800 & 1.1 & 4.35589 & 2.0 \\
\hline 23 & $32706-3-24$ & 170 & 31125 & 1.7 & 4.23235 & 1.5 \\
\hline 23 & 32706-3-15 & 63 & 13571 & 1.3 & 4.00598 & 4.3 \\
\hline 23 & $32706-3-6$ & 362 & 56414 & 2.1 & 4.41493 & 1.7 \\
\hline 23 & 32706-3-39 & 203 & 36764 & 1.7 & 4.34459 & 3.1 \\
\hline 23 & $32706-3-40$ & 210 & 44348 & 1.7 & 4.37457 & 3.6 \\
\hline 23 & 32706-3-55 & 546 & 71371 & 0.8 & 4.33378 & 3.6 \\
\hline 23 & $32706-3-21$ & 406 & 71582 & 1.9 & 4.52605 & 2.1 \\
\hline 23 & $32706-3-10$ & 388 & 32914 & 3.2 & 4.32578 & 3.3 \\
\hline 23 & $32706-3-52$ & 499 & 77771 & 7.1 & 4.21373 & 2.8 \\
\hline 23 & $32706-3-5$ & 245 & 37374 & 1.5 & 4.56543 & 3.4 \\
\hline 23 & $32706-3-42$ & 118 & 21468 & 1.4 & 4.70385 & 4.8 \\
\hline
\end{tabular}


Kimbrough, D.L., Grove, M., Gehrels, G.E., Dorsey, R.J., Howard, K.A., Lovera, O., Aslan, A., House, P.K., and Pearthree, P.A., 2015, Detrital zircon U-Pb provenance of the Colorado River: A 5 m.y. record of incision into cover strata overlying the Colorado Plateau and adjacent regions: Geosphere, v. 11, doi:10.1130/GES00982.1.

TABLE DR7. U-Pb ZIRCON LA-ICP-MS GEOCHRONOLOGIC ANALYSES OF COLORADO RIVER BASIN \& ASSOCIATED SAMPLES.

\begin{tabular}{|c|c|c|c|c|c|c|}
\hline & & & & & & opic ra \\
\hline ID\# & Analysis & $\underset{(\mathrm{ppm})}{U}$ & $\begin{array}{l}206 \mathrm{~Pb} \\
204 \mathrm{~Pb}\end{array}$ & $\mathrm{U} / \mathrm{Th}$ & $\begin{array}{c}207 \mathrm{~Pb}^{*} \\
235 \mathrm{U}\end{array}$ & $\begin{array}{c} \pm \\
(\%)\end{array}$ \\
\hline 24 & $32306-175-50$ & 259 & 450 & 0.2 & 0.00957 & 29.7 \\
\hline 24 & $32306-175-37$ & 1194 & 1288 & 0.5 & 0.01661 & 6.5 \\
\hline 24 & $32306-175-34$ & 950 & 1258 & 0.5 & 0.01626 & 7.8 \\
\hline 24 & $32306-175-35$ & 1613 & 1429 & 0.5 & 0.01809 & 7.0 \\
\hline 24 & $32306-175-6$ & 223 & 362 & 0.5 & 0.00888 & 21.2 \\
\hline 24 & $32306-175-33$ & 205 & 1975 & 0.5 & 0.17672 & 9.1 \\
\hline 24 & $32306-175-49$ & 103 & 10252 & 1.0 & 3.79440 & 2.6 \\
\hline 24 & $32306-175-56$ & 145 & 8213 & 2.2 & 3.24442 & 2.5 \\
\hline 24 & $32306-175-59$ & 259 & 49466 & 4.0 & 4.19184 & 3.3 \\
\hline 24 & $32306-175-57$ & 362 & 46463 & 3.4 & 4.10314 & 3.4 \\
\hline 24 & $32306-175-53$ & 521 & 63779 & 2.8 & 4.12338 & 4.1 \\
\hline 24 & $32306-175-41$ & 46 & 11228 & 1.6 & 4.09837 & 3.7 \\
\hline 24 & $32306-175-47$ & 333 & 52158 & 2.6 & 4.35987 & 1.9 \\
\hline 24 & $32306-175-1$ & 281 & 43733 & 3.2 & 4.23036 & 2.2 \\
\hline 24 & $32306-175-9$ & 316 & 40983 & 2.7 & 4.20677 & 3.5 \\
\hline 24 & $32306-175-55$ & 238 & 44704 & 1.5 & 4.10112 & 3.5 \\
\hline 24 & $32306-175-26$ & 229 & 25937 & 1.2 & 4.44559 & 3.0 \\
\hline 24 & $32306-175-39$ & 172 & 26286 & 1.5 & 4.30284 & 1.5 \\
\hline 24 & $32306-175-43$ & 138 & 30204 & 2.7 & 4.27821 & 2.5 \\
\hline 24 & $32306-175-5$ & 90 & 15411 & 0.9 & 4.22264 & 2.7 \\
\hline 24 & $32306-175-54$ & 201 & 40299 & 2.8 & 4.37161 & 2.8 \\
\hline 24 & $32306-175-32$ & 197 & 41516 & 3.0 & 4.48073 & 2.3 \\
\hline 24 & $32306-175-27$ & 237 & 35209 & 2.5 & 4.13303 & 2.5 \\
\hline 24 & $32306-175-25$ & 166 & 22235 & 1.8 & 4.22927 & 2.3 \\
\hline 24 & $32306-175-52$ & 301 & 69652 & 5.1 & 4.26143 & 8.2 \\
\hline 24 & $32306-175-12$ & 176 & 40936 & 2.1 & 4.51730 & 4.1 \\
\hline 24 & $32306-175-11$ & 108 & 20013 & 1.9 & 4.46292 & 3.3 \\
\hline 24 & $32306-175-42$ & 141 & 33351 & 1.3 & 4.39477 & 2.8 \\
\hline 24 & $32306-175-16$ & 339 & 44374 & 1.9 & 4.47198 & 2.0 \\
\hline 24 & $32306-175-8$ & 328 & 68669 & 2.0 & 4.20780 & 2.6 \\
\hline 24 & $32306-175-48$ & 302 & 57546 & 1.4 & 4.12658 & 3.7 \\
\hline 24 & $32306-175-24$ & 282 & 43980 & 6.1 & 4.23010 & 2.6 \\
\hline 24 & $32306-175-20$ & 103 & 17429 & 1.4 & 4.22772 & 2.2 \\
\hline 24 & $32306-175-3$ & 280 & 38533 & 1.7 & 4.22229 & 1.8 \\
\hline 24 & $32306-175-15$ & 841 & 100428 & 2.6 & 4.05594 & 6.8 \\
\hline 24 & $32306-175-51$ & 144 & 25556 & 1.4 & 4.22778 & 2.0 \\
\hline 24 & $32306-175-58$ & 411 & 59215 & 5.9 & 4.11870 & 2.2 \\
\hline 24 & $32306-175-22$ & 84 & 10379 & 1.2 & 4.19982 & 2.8 \\
\hline 24 & $32306-175-18$ & 258 & 45187 & 3.1 & 4.41624 & 3.1 \\
\hline 24 & $32306-175-45$ & 149 & 32061 & 2.0 & 3.89435 & 2.4 \\
\hline 24 & $32306-175-14$ & 437 & 62154 & 9.9 & 4.25578 & 2.5 \\
\hline 24 & $32306-175-30$ & 100 & 11604 & 1.2 & 4.26879 & 3.7 \\
\hline 24 & $32306-175-36$ & 356 & 14227 & 1.8 & 4.25707 & 5.4 \\
\hline 24 & $32306-175-13$ & 273 & 40633 & 2.3 & 4.36202 & 2.7 \\
\hline 24 & $32306-175-21$ & 239 & 45387 & 3.1 & 4.15747 & 2.1 \\
\hline 24 & $32306-175-10$ & 240 & 37236 & 3.8 & 4.24043 & 3.4 \\
\hline 24 & $32306-175-44$ & 324 & 50059 & 5.1 & 4.18552 & 3.5 \\
\hline 24 & $32306-175-40$ & 216 & 34860 & 1.3 & 4.38764 & 2.1 \\
\hline 24 & $32306-175-28$ & 221 & 41199 & 4.8 & 4.53617 & 2.2 \\
\hline 24 & $32306-175-23$ & 237 & 38223 & 2.1 & 4.51634 & 2.3 \\
\hline 24 & $32306-175-46$ & 487 & 72731 & 1.6 & 4.26089 & 1.9 \\
\hline 24 & $32306-175-60$ & 338 & 44933 & 1.8 & 4.46520 & 2.7 \\
\hline 24 & $32306-175-19$ & 1170 & 34290 & 3.5 & 4.40433 & 4.7 \\
\hline 25 & 325061-32 & 389 & 509 & 0.3 & 0.01949 & 15.4 \\
\hline 25 & $325061-45$ & 327 & 372 & 0.7 & 0.02384 & 18.7 \\
\hline 25 & $325061-23$ & 191 & 323 & 0.8 & 0.01951 & 28.1 \\
\hline 25 & $325061-42$ & 714 & 3398 & 1.4 & 0.07881 & 6.6 \\
\hline 25 & $325061-18$ & 250 & 1546 & 1.1 & 0.08986 & 10.3 \\
\hline 25 & $325061-26$ & 1005 & 100900 & 1.9 & 3.82110 & 5.4 \\
\hline 25 & $325061-19$ & 166 & 29659 & 1.1 & 4.24938 & 1.6 \\
\hline 25 & $325061-21$ & 745 & 102216 & 1.6 & 4.32907 & 2.9 \\
\hline 25 & $325061-20$ & 303 & 56531 & 1.9 & 4.28901 & 1.5 \\
\hline 25 & $325061-55$ & 527 & 23937 & 1.3 & 4.14678 & 2.4 \\
\hline 25 & $325061-54$ & 232 & 24355 & 2.3 & 4.36532 & 3.6 \\
\hline 25 & $325061-36$ & 170 & 24368 & 1.7 & 4.13205 & 2.0 \\
\hline 25 & $325061-29$ & 266 & 29431 & 1.7 & 4.28006 & 2.8 \\
\hline 25 & $325061-38$ & 217 & 15954 & 2.0 & 4.07291 & 2.4 \\
\hline 25 & $325061-15$ & 249 & 43446 & 2.1 & 4.23096 & 2.4 \\
\hline 25 & $325061-22$ & 615 & 87415 & 2.2 & 4.22519 & 2.1 \\
\hline 25 & $325061-39$ & 245 & 37323 & 1.6 & 4.39369 & 1.5 \\
\hline 25 & $325061-28$ & 251 & 17787 & 1.8 & 4.23640 & 4.0 \\
\hline 25 & $325061-50$ & 283 & 33872 & 1.0 & 4.38984 & 5.4 \\
\hline 25 & $325061-14$ & 125 & 20238 & 1.2 & 4.23961 & 1.9 \\
\hline 25 & $325061-41$ & 233 & 33751 & 1.5 & 4.53133 & 4.9 \\
\hline 25 & $325061-43$ & 1149 & 112746 & 2.4 & 4.12778 & 1.4 \\
\hline 25 & $325061-35$ & 87 & 13882 & 1.4 & 4.22974 & 1.4 \\
\hline 25 & $325061-3$ & 243 & 34014 & 1.6 & 4.40502 & 2.3 \\
\hline 25 & $325061-48$ & 236 & 29812 & 1.5 & 4.33487 & 1.9 \\
\hline 25 & $325061-16$ & 1848 & 146051 & 7.3 & 4.44553 & 2.4 \\
\hline 25 & $325061-12$ & 131 & 23798 & 1.8 & 4.29894 & 1.7 \\
\hline 25 & $325061-9$ & 258 & 41931 & 2.2 & 4.63188 & 2.6 \\
\hline 25 & $325061-10$ & 158 & 12268 & 1.4 & 4.41587 & 3.8 \\
\hline 25 & $325061-53$ & 156 & 3794 & 1.9 & 4.45865 & 2.1 \\
\hline 25 & $325061-37$ & 235 & 33259 & 2.1 & 4.50512 & 1.4 \\
\hline 25 & $325061-44$ & 321 & 7257 & 1.8 & 4.64152 & 7.4 \\
\hline 25 & $325061-47$ & 685 & 59545 & 4.1 & 4.54764 & 4.7 \\
\hline 26 & $32103-1-16$ & 490 & 1727 & 1.0 & 0.02717 & 16.8 \\
\hline 26 & $32103-1-12$ & 447 & 1180 & 0.8 & 0.03069 & 15.0 \\
\hline 26 & 32103-1-14 & 376 & 560 & 0.8 & 0.06013 & 13.4 \\
\hline 26 & $32103-1-30$ & 129 & 700 & 1.1 & 0.04103 & 30.6 \\
\hline
\end{tabular}


Kimbrough, D.L., Grove, M., Gehrels, G.E., Dorsey, R.J., Howard, K.A., Lovera, O., Aslan, A., House, P.K., and Pearthree, P.A., 2015, Detrital zircon U-Pb provenance of the Colorado River: A 5 m.y. record of incision into cover strata overlying the Colorado Plateau and adjacent regions: Geosphere, v. 11, doi:10.1130/GES00982.1.

TABLE DR7. U-Pb ZIRCON LA-ICP-MS GEOCHRONOLOGIC ANALYSES OF COLORADO RIVER BASIN \& ASSOCIATED SAMPLES.

\begin{tabular}{|c|c|c|c|c|c|c|}
\hline & & & & & & opic ra \\
\hline ID\# & Analysis & $\begin{array}{c}\text { U } \\
(\mathrm{ppm})\end{array}$ & $\begin{array}{l}206 \mathrm{~Pb} \\
204 \mathrm{~Pb} \\
\end{array}$ & U/Th & $\begin{array}{c}207 \mathrm{~Pb}^{*} \\
235 \mathrm{U}\end{array}$ & $\begin{array}{c} \pm \\
(\%)\end{array}$ \\
\hline 26 & $32103-1-75$ & 182 & 1088 & 2.1 & 0.06926 & 25.7 \\
\hline 26 & 32103-1-31 & 105 & 384 & 0.8 & 0.05334 & 23.4 \\
\hline 26 & 32103-1-55 & 623 & 2894 & 0.6 & 0.04807 & 19.6 \\
\hline 26 & 32103-1-38 & 252 & 1419 & 2.0 & 0.04170 & 24.5 \\
\hline 26 & $32103-1-33$ & 206 & 2379 & 1.3 & 0.07750 & 14.0 \\
\hline 26 & $32103-1-24$ & 1099 & 14507 & 2.7 & 0.10767 & 3.2 \\
\hline 26 & $32103-1-65$ & 453 & 12670 & 1.2 & 0.17504 & 3.6 \\
\hline 26 & 32103-1-35 & 125 & 3003 & 1.2 & 0.18632 & 13.5 \\
\hline 26 & $32103-1-4$ & 378 & 8429 & 1.5 & 0.19019 & 3.8 \\
\hline 26 & $32103-1-56$ & 527 & 18479 & 1.1 & 0.24488 & 3.6 \\
\hline 26 & $32103-1-61$ & 194 & 9565 & 1.4 & 0.25989 & 5.1 \\
\hline 26 & $32103-1-58$ & 372 & 12911 & 0.9 & 0.29546 & 5.4 \\
\hline 26 & $32103-1-44$ & 1046 & 13228 & 1.8 & 0.42080 & 6.6 \\
\hline 26 & $32103-1-67$ & 140 & 9525 & 1.2 & 0.58725 & 5.3 \\
\hline 26 & $32103-1-18$ & 45 & 3064 & 0.9 & 0.59126 & 6.1 \\
\hline 26 & $32103-1-69$ & 87 & 7626 & 1.3 & 0.71750 & 4.8 \\
\hline 26 & 32103-1-LS14 & 458 & 46370 & 3.5 & 0.75621 & 2.0 \\
\hline 26 & $32103-1-28$ & 99 & 7157 & 0.8 & 0.85165 & 6.3 \\
\hline 26 & $32103-1-20$ & 167 & 24193 & 1.9 & 1.64901 & 2.8 \\
\hline 26 & $32103-1-29$ & 47 & 7768 & 3.3 & 1.74715 & 3.8 \\
\hline 26 & $32103-1-10$ & 51 & 8446 & 2.1 & 1.71740 & 2.8 \\
\hline 26 & $32103-1-51$ & 126 & 19297 & 1.1 & 1.73505 & 2.2 \\
\hline 26 & $32103-1-66$ & 115 & 22180 & 2.2 & 1.86660 & 2.8 \\
\hline 26 & $32103-1-21$ & 72 & 11261 & 2.8 & 1.79665 & 2.8 \\
\hline 26 & 32103-1-42 & 152 & 26260 & 2.1 & 1.87310 & 2.8 \\
\hline 26 & $32103-1-43$ & 72 & 15454 & 1.6 & 1.89811 & 3.8 \\
\hline 26 & 32103-1-36 & 340 & 19527 & 1.1 & 1.64009 & 5.7 \\
\hline 26 & $32103-1-64$ & 155 & 27834 & 0.8 & 1.90175 & 1.5 \\
\hline 26 & 32103-1-39 & 242 & 20986 & 3.2 & 1.97574 & 8.5 \\
\hline 26 & $32103-1-71$ & 193 & 44064 & 2.8 & 1.99778 & 2.7 \\
\hline 26 & $32103-1-63$ & 49 & 12338 & 1.5 & 2.00041 & 3.0 \\
\hline 26 & $32103-1-49$ & 37 & 5460 & 0.9 & 1.87445 & 4.3 \\
\hline 26 & 32103-1-25 & 156 & 27960 & 1.0 & 2.88930 & 1.6 \\
\hline 26 & $32103-1-17$ & 120 & 25791 & 1.3 & 2.90394 & 1.5 \\
\hline 26 & $32103-1-8$ & 33 & 7389 & 1.1 & 2.96353 & 2.9 \\
\hline 26 & $32103-1-3$ & 59 & 14146 & 0.6 & 3.02895 & 1.8 \\
\hline 26 & $32103-1-53$ & 119 & 18270 & 0.9 & 2.87918 & 1.9 \\
\hline 26 & $32103-1-60$ & 308 & 102207 & 1.8 & 3.12470 & 2.1 \\
\hline 26 & $32103-1-26$ & 184 & 40144 & 1.4 & 3.15224 & 2.4 \\
\hline 26 & $32103-1-46$ & 427 & 3887 & 2.2 & 2.23980 & 4.4 \\
\hline 26 & $32103-1-68$ & 74 & 12245 & 1.5 & 3.30773 & 5.5 \\
\hline 26 & $32103-1-32$ & 155 & 21475 & 0.7 & 3.36113 & 3.3 \\
\hline 26 & $32103-1-72$ & 551 & 125543 & 1.8 & 3.47903 & 1.5 \\
\hline 26 & $32103-1-52$ & 1049 & 79850 & 6.3 & 3.75945 & 2.7 \\
\hline 26 & $32103-1-1$ & 29 & 7625 & 1.1 & 3.91432 & 3.4 \\
\hline 26 & $32103-1-23$ & 126 & 33242 & 2.5 & 4.10654 & 2.5 \\
\hline 26 & 32103-1-19 & 104 & 24364 & 1.0 & 4.52902 & 3.1 \\
\hline 26 & $32103-1-48$ & 1427 & 143803 & 13.1 & 3.94693 & 5.1 \\
\hline 26 & $32103-1-6$ & 224 & 37229 & 0.9 & 4.27589 & 5.5 \\
\hline 26 & $32103-1-70$ & 45 & 18855 & 1.2 & 4.68160 & 6.5 \\
\hline 26 & $32103-1-41$ & 161 & 43670 & 1.1 & 4.53853 & 2.0 \\
\hline 26 & $32103-1-7$ & 136 & 26795 & 1.8 & 4.71036 & 3.5 \\
\hline 26 & $32103-1-9$ & 81 & 24349 & 2.8 & 4.41167 & 2.3 \\
\hline 26 & $32103-1-62$ & 183 & 12818 & 2.6 & 4.45302 & 1.7 \\
\hline 26 & $32103-1-50$ & 70 & 26167 & 2.1 & 4.96778 & 3.0 \\
\hline 26 & $32103-1-5$ & 240 & 64939 & 1.6 & 4.89309 & 2.2 \\
\hline 26 & $32103-1-22$ & 69 & 20403 & 4.2 & 4.99976 & 2.1 \\
\hline 26 & $32103-1-13$ & 230 & 43979 & 2.0 & 4.61386 & 2.8 \\
\hline 26 & $32103-1-59$ & 332 & 98708 & 2.6 & 5.43200 & 1.6 \\
\hline 26 & $32103-1-40$ & 9 & 479 & 14.3 & 1.53870 & 19.9 \\
\hline 26 & 32103-1-11 & 255 & 52181 & 3.0 & 5.32462 & 5.3 \\
\hline 26 & $32103-1-34$ & 174 & 54725 & 2.8 & 6.76038 & 2.4 \\
\hline 26 & $32103-1-27$ & 65 & 23389 & 1.1 & 6.70905 & 1.5 \\
\hline 26 & $32103-1-2$ & 244 & 59845 & 1.3 & 12.10250 & 1.6 \\
\hline 26 & 32103-1-15 & 90 & 32455 & 0.7 & 12.68526 & 2.6 \\
\hline 26 & 32103-1-45 & 487 & 226180 & 6.6 & 15.67101 & 5.1 \\
\hline 26 & $32103-1-73$ & 358 & 200200 & 1.1 & 18.25722 & 2.4 \\
\hline 27 & 32003-13-25 & 1902 & 3029 & 0.4 & 0.02055 & 11.1 \\
\hline 27 & $32003-13-40$ & 170 & 347 & 1.0 & 0.03565 & 26.3 \\
\hline 27 & $32003-13-74$ & 264 & 347 & 0.5 & 0.02338 & 35.4 \\
\hline 27 & $32003-13-31$ & 228 & 487 & 0.6 & 0.02235 & 31.0 \\
\hline 27 & 32003-13-59 & 482 & 1764 & 1.1 & 0.03826 & 14.9 \\
\hline 27 & 32003-13-1 & 122 & 610 & 1.8 & 0.06257 & 21.0 \\
\hline 27 & $32003-13-41$ & 273 & 1534 & 1.2 & 0.05914 & 13.8 \\
\hline 27 & $32003-13-53$ & 444 & 2213 & 0.5 & 0.09252 & 9.4 \\
\hline 27 & 32003-13-35 & 227 & 1756 & 0.7 & 0.10808 & 9.5 \\
\hline 27 & $32003-13-44$ & 111 & 1237 & 1.3 & 0.11518 & 30.0 \\
\hline 27 & $32003-13-73$ & 496 & 6735 & 0.8 & 0.13025 & 6.6 \\
\hline 27 & $32003-13-47$ & 692 & 7638 & 1.5 & 0.18342 & 2.8 \\
\hline 27 & $32003-13-60$ & 587 & 1119 & 0.7 & 0.39572 & 13.1 \\
\hline 27 & 32003-13-32 & 29 & 760 & 0.8 & 0.66013 & 25.1 \\
\hline 27 & $32003-13-27$ & 664 & 2151 & 0.5 & 0.66685 & 2.2 \\
\hline 27 & $32003-13-19$ & 362 & 6973 & 1.9 & 0.56938 & 5.3 \\
\hline 27 & $32003-13-12$ & 525 & 2474 & 3.0 & 0.69243 & 6.2 \\
\hline 27 & $32003-13-64$ & 268 & 12289 & 0.9 & 0.55958 & 2.8 \\
\hline 27 & $32003-13-15$ & 209 & 5840 & 2.4 & 0.64300 & 3.5 \\
\hline 27 & $32003-13-52$ & 193 & 3825 & 1.1 & 0.71000 & 3.8 \\
\hline 27 & $32003-13-34$ & 96 & 3252 & 1.0 & 0.92491 & 11.7 \\
\hline 27 & $32003-13-23$ & 53 & 1737 & 0.9 & 1.11135 & 9.4 \\
\hline 27 & $32003-13-7$ & 392 & 31840 & 3.1 & 0.87664 & 3.0 \\
\hline
\end{tabular}


Kimbrough, D.L., Grove, M., Gehrels, G.E., Dorsey, R.J., Howard, K.A., Lovera, O., Aslan, A., House, P.K., and Pearthree, P.A., 2015, Detrital zircon U-Pb provenance of the Colorado River: A 5 m.y. record of incision into cover strata overlying the Colorado Plateau and adjacent regions: Geosphere, v. 11, doi:10.1130/GES00982.1.

TABLE DR7. U-Pb ZIRCON LA-ICP-MS GEOCHRONOLOGIC ANALYSES OF COLORADO RIVER BASIN \& ASSOCIATED SAMPLES.

\begin{tabular}{|c|c|c|c|c|c|c|}
\hline & & & & & & opic ra \\
\hline ID\# & Analysis & $\begin{array}{c}\text { U } \\
(\mathrm{ppm})\end{array}$ & $\begin{array}{l}206 \mathrm{~Pb} \\
204 \mathrm{~Pb}\end{array}$ & $\mathrm{U} / \mathrm{Th}$ & $\begin{array}{c}207 \mathrm{~Pb}^{*} \\
235 \mathrm{U}\end{array}$ & $\begin{array}{c} \pm \\
(\%) \\
\end{array}$ \\
\hline 27 & $32003-13-68$ & 199 & 15574 & 3.2 & 1.44843 & 4.3 \\
\hline 27 & $32003-13-5$ & 185 & 23959 & 1.1 & 1.70541 & 2.2 \\
\hline 27 & $32003-13-69$ & 351 & 26624 & 2.8 & 1.80518 & 1.6 \\
\hline 27 & $32003-13-26$ & 271 & 26113 & 3.6 & 1.90213 & 2.3 \\
\hline 27 & $32003-13-48$ & 363 & 19216 & 2.8 & 2.02589 & 1.5 \\
\hline 27 & $32003-13-28$ & 76 & 11006 & 1.3 & 2.72673 & 2.7 \\
\hline 27 & $32003-13-10$ & 103 & 19941 & 1.3 & 2.94476 & 3.0 \\
\hline 27 & $32003-13-43$ & 65 & 5396 & 0.5 & 2.93365 & 3.1 \\
\hline 27 & $32003-13-62$ & 79 & 8584 & 1.3 & 3.32992 & 3.4 \\
\hline 27 & $32003-13-51$ & 675 & 28293 & 14.4 & 3.68864 & 4.0 \\
\hline 27 & $32003-13-9$ & 1152 & 214760 & 12.8 & 4.14677 & 2.1 \\
\hline 27 & $32003-13-38$ & 136 & 30433 & 1.4 & 4.42201 & 3.9 \\
\hline 27 & $32003-13-58$ & 182 & 20893 & 2.2 & 4.32301 & 1.6 \\
\hline 27 & $32003-13-67$ & 709 & 96273 & 1.3 & 4.12289 & 1.7 \\
\hline 27 & $32003-13-8$ & 256 & 56324 & 1.5 & 4.35826 & 2.1 \\
\hline 27 & $32003-13-39$ & 45 & 5004 & 1.0 & 3.69257 & 3.3 \\
\hline 27 & $32003-13-4$ & 157 & 36798 & 1.8 & 4.29438 & 2.8 \\
\hline 27 & $32003-13-3$ & 304 & 64573 & 4.1 & 3.94950 & 3.0 \\
\hline 27 & $32003-13-2$ & 301 & 72981 & 3.3 & 4.39425 & 3.0 \\
\hline 27 & $32003-13-18$ & 425 & 17487 & 1.5 & 3.65829 & 4.0 \\
\hline 27 & $32003-13-20$ & 178 & 21417 & 1.1 & 4.05849 & 4.1 \\
\hline 27 & $32003-13-55$ & 410 & 32045 & 1.7 & 3.89412 & 8.2 \\
\hline 27 & $32003-13-6$ & 263 & 44627 & 0.8 & 4.18413 & 2.8 \\
\hline 27 & $32003-13-72$ & 237 & 22647 & 2.4 & 4.31280 & 1.7 \\
\hline 27 & $32003-13-22$ & 109 & 11072 & 1.2 & 3.71062 & 3.1 \\
\hline 27 & $32003-13-56$ & 597 & 49504 & 5.0 & 4.02525 & 3.4 \\
\hline 27 & 32003-13-37 & 463 & 75581 & 0.9 & 4.15957 & 3.9 \\
\hline 27 & $32003-13-71$ & 113 & 17883 & 1.0 & 4.35359 & 3.2 \\
\hline 27 & $32003-13-54$ & 762 & 59639 & 1.1 & 4.32627 & 2.9 \\
\hline 27 & $32003-13-65$ & 1109 & 72679 & 1.1 & 3.85947 & 6.2 \\
\hline 27 & $32003-13-75$ & 679 & 74591 & 1.5 & 4.32054 & 2.9 \\
\hline 27 & $32003-13-30$ & 381 & 32096 & 2.6 & 4.09004 & 3.0 \\
\hline 27 & $32003-13-16$ & 233 & 11337 & 2.6 & 4.50994 & 1.8 \\
\hline 27 & $32003-13-24$ & 470 & 72611 & 1.9 & 4.44280 & 2.6 \\
\hline 27 & $32003-13-45$ & 102 & 6319 & 1.7 & 3.92699 & 2.9 \\
\hline 27 & $32003-13-42$ & 39 & 4403 & 0.4 & 3.91601 & 2.6 \\
\hline 27 & $32003-13-33$ & 53 & 5034 & 0.5 & 4.45259 & 4.0 \\
\hline 27 & $32003-13-14$ & 182 & 9728 & 1.3 & 4.35091 & 4.9 \\
\hline 27 & $32003-13-21$ & 116 & 17522 & 1.2 & 4.53012 & 1.9 \\
\hline 27 & $32003-13-70$ & 143 & 6882 & 1.2 & 4.63244 & 3.8 \\
\hline 27 & $32003-13-11$ & 225 & 25653 & 1.6 & 4.61251 & 2.4 \\
\hline 27 & $32003-13-46$ & 63 & 3785 & 1.3 & 4.52307 & 2.6 \\
\hline 27 & $32003-13-57$ & 203 & 32171 & 1.2 & 4.72073 & 10.6 \\
\hline 27 & $32003-13-50$ & 76 & 11140 & 1.8 & 4.45025 & 4.0 \\
\hline 27 & $32003-13-29$ & 103 & 8771 & 1.2 & 4.74619 & 4.5 \\
\hline 27 & $32003-13-49$ & 374 & 22141 & 2.1 & 11.39283 & 7.0 \\
\hline 28 & $32003-7-22$ & 566 & 1509 & 0.5 & 0.04267 & 28.7 \\
\hline 28 & $32003-7-72$ & 3112 & 10113 & 1.8 & 0.03807 & 3.8 \\
\hline 28 & $32003-7-7$ & 79 & 725 & 1.4 & 0.04823 & 39.0 \\
\hline 28 & $32003-7-66$ & 832 & 9481 & 1.6 & 0.09129 & 5.3 \\
\hline 28 & $32003-7-69$ & 349 & 2351 & 2.0 & 0.10521 & 13.7 \\
\hline 28 & $32003-7-40$ & 347 & 1841 & 2.7 & 0.12253 & 20.8 \\
\hline 28 & $32003-7-18$ & 345 & 4445 & 2.1 & 0.11855 & 6.0 \\
\hline 28 & $32003-7-33$ & 160 & 1854 & 2.4 & 0.13462 & 17.9 \\
\hline 28 & $32003-7-60$ & 827 & 4154 & 0.9 & 0.16556 & 3.9 \\
\hline 28 & $32003-7-36$ & 477 & 5269 & 1.2 & 0.21076 & 7.1 \\
\hline 28 & $32003-7-4$ & 232 & 2313 & 1.6 & 0.30022 & 19.9 \\
\hline 28 & $32003-7-13$ & 511 & 12517 & 0.8 & 0.24707 & 6.3 \\
\hline 28 & $32003-7-67$ & 94 & 2964 & 1.2 & 0.27745 & 6.4 \\
\hline 28 & $32003-7-21$ & 221 & 12766 & 2.0 & 0.73180 & 3.7 \\
\hline 28 & $32003-7-44$ & 242 & 9421 & 2.0 & 0.80648 & 3.4 \\
\hline 28 & $32003-7-49$ & 606 & 23033 & 1.1 & 1.09347 & 2.9 \\
\hline 28 & $32003-7-59$ & 334 & 30975 & 1.8 & 1.23989 & 3.6 \\
\hline 28 & $32003-7-43$ & 69 & 9318 & 4.9 & 1.74241 & 3.7 \\
\hline 28 & $32003-7-17$ & 92 & 10279 & 1.1 & 1.87904 & 3.9 \\
\hline 28 & $32003-7-62$ & 159 & 24369 & 3.3 & 2.09716 & 2.3 \\
\hline 28 & $32003-7-41$ & 581 & 20871 & 10.2 & 2.07156 & 1.7 \\
\hline 28 & $32003-7-15$ & 136 & 25260 & 1.1 & 2.20390 & 2.5 \\
\hline 28 & $32003-7-58$ & 275 & 32116 & 1.7 & 2.46343 & 2.0 \\
\hline 28 & $32003-7-54$ & 247 & 39210 & 1.9 & 2.91852 & 2.9 \\
\hline 28 & $32003-7-29$ & 164 & 23357 & 3.1 & 3.09450 & 1.7 \\
\hline 28 & $32003-7-70$ & 261 & 58789 & 2.5 & 2.97664 & 1.9 \\
\hline 28 & $32003-7-14$ & 36 & 6583 & 0.5 & 2.96518 & 4.5 \\
\hline 28 & $32003-7-16$ & 288 & 42336 & 0.9 & 3.07036 & 1.5 \\
\hline 28 & $32003-7-37$ & 234 & 32985 & 1.3 & 3.24785 & 1.9 \\
\hline 28 & $32003-7-10$ & 156 & 26429 & 2.4 & 3.16419 & 2.7 \\
\hline 28 & $32003-7-56$ & 120 & 21301 & 1.3 & 3.18927 & 5.2 \\
\hline 28 & $32003-7-31$ & 278 & 22657 & 1.4 & 3.14113 & 2.0 \\
\hline 28 & $32003-7-73$ & 320 & 15338 & 1.7 & 3.38604 & 3.0 \\
\hline 28 & $32003-7-1$ & 52 & 7593 & 0.8 & 3.09480 & 2.4 \\
\hline 28 & $32003-7-32$ & 177 & 24790 & 2.2 & 3.01516 & 6.0 \\
\hline 28 & $32003-7-35$ & 446 & 19556 & 2.3 & 2.76178 & 7.2 \\
\hline 28 & $32003-7-51$ & 49 & 3061 & 0.8 & 3.20374 & 5.6 \\
\hline 28 & $32003-7-75$ & 227 & 35006 & 1.2 & 3.72538 & 2.4 \\
\hline 28 & $32003-7-25$ & 904 & 70578 & 3.6 & 3.04085 & 6.4 \\
\hline 28 & $32003-7-39$ & 43 & 9842 & 1.3 & 4.34880 & 3.1 \\
\hline 28 & $32003-7-61$ & 408 & 22550 & 1.2 & 3.43655 & 7.7 \\
\hline 28 & $32003-7-11$ & 264 & 31275 & 2.0 & 3.96143 & 3.3 \\
\hline 28 & $32003-7-68$ & 126 & 24727 & 0.8 & 3.76505 & 2.9 \\
\hline 28 & $32003-7-42$ & 576 & 68628 & 2.4 & 3.87606 & 1.7 \\
\hline
\end{tabular}


Kimbrough, D.L., Grove, M., Gehrels, G.E., Dorsey, R.J., Howard, K.A., Lovera, O., Aslan, A., House, P.K., and Pearthree, P.A., 2015, Detrital zircon U-Pb provenance of the Colorado River: A 5 m.y. record of incision into cover strata overlying the Colorado Plateau and adjacent regions: Geosphere, v. 11, doi:10.1130/GES00982.1.

TABLE DR7. U-Pb ZIRCON LA-ICP-MS GEOCHRONOLOGIC ANALYSES OF COLORADO RIVER BASIN \& ASSOCIATED SAMPLES.

\begin{tabular}{|c|c|c|c|c|c|c|}
\hline & & & & & & opic ra \\
\hline ID\# & Analysis & $\begin{array}{c}\text { U } \\
(\mathrm{ppm})\end{array}$ & $\begin{array}{l}206 \mathrm{~Pb} \\
204 \mathrm{~Pb}\end{array}$ & $\mathrm{U} / \mathrm{Th}$ & $\begin{array}{c}207 \mathrm{~Pb}^{*} \\
235 \mathrm{U}\end{array}$ & $\begin{array}{c} \pm \\
(\%) \\
\end{array}$ \\
\hline 28 & $32003-7-8$ & 155 & 37034 & 1.7 & 4.31667 & 2.0 \\
\hline 28 & $32003-7-46$ & 69 & 17687 & 1.0 & 4.17492 & 1.9 \\
\hline 28 & $32003-7-74$ & 293 & 67529 & 2.7 & 4.07973 & 1.5 \\
\hline 28 & $32003-7-57$ & 261 & 13446 & 1.7 & 3.82467 & 5.2 \\
\hline 28 & $32003-7-34$ & 745 & 143228 & 7.2 & 4.58168 & 3.1 \\
\hline 28 & $32003-7-30$ & 251 & 50093 & 1.7 & 4.61237 & 2.0 \\
\hline 28 & $32003-7-38$ & 23 & 3417 & 0.7 & 4.58293 & 5.4 \\
\hline 28 & $32003-7-55$ & 604 & 18562 & 4.3 & 3.87445 & 5.2 \\
\hline 28 & $32003-7-19$ & 338 & 43123 & 0.9 & 4.41449 & 4.8 \\
\hline 28 & $32003-7-28$ & 2106 & 53164 & 1.6 & 4.38256 & 2.6 \\
\hline 28 & $32003-7-20$ & 487 & 42786 & 1.4 & 4.44440 & 1.5 \\
\hline 28 & $32003-7-71$ & 156 & 9922 & 0.6 & 4.09383 & 2.7 \\
\hline 28 & $32003-7-2$ & 455 & 86380 & 3.7 & 4.73585 & 3.0 \\
\hline 28 & $32003-7-65$ & 96 & 23706 & 2.9 & 4.68712 & 1.7 \\
\hline 28 & $32003-7-6$ & 73 & 21734 & 1.0 & 5.31968 & 1.4 \\
\hline 28 & $32003-7-5$ & 30 & 1766 & 1.2 & 3.88300 & 4.4 \\
\hline 28 & $32003-7-47$ & 43 & 821 & 0.8 & 2.31556 & 17.9 \\
\hline 29 & $06322-6-24$ & 923 & 1176 & 0.5 & 0.02042 & 9.0 \\
\hline 29 & $06322-6-4$ & 293 & 1120 & 0.7 & 0.03436 & 11.8 \\
\hline 29 & $06322-6-13$ & 238 & 1230 & 0.7 & 0.09548 & 12.7 \\
\hline 29 & $06322-6-28$ & 80 & 1275 & 0.4 & 0.16314 & 12.8 \\
\hline 29 & 06322-6-39 & 234 & 4293 & 1.1 & 0.19820 & 7.1 \\
\hline 29 & $06322-6-45$ & 919 & 12617 & 3.8 & 0.18988 & 1.8 \\
\hline 29 & $06322-6-7$ & 838 & 8402 & 0.6 & 0.49582 & 3.5 \\
\hline 29 & 06322-6-49 & 166 & 3959 & 1.1 & 0.49730 & 4.7 \\
\hline 29 & $06322-6-14$ & 292 & 8400 & 1.3 & 0.50497 & 4.3 \\
\hline 29 & 06322-6-32 & 185 & 11078 & 0.5 & 0.54246 & 2.2 \\
\hline 29 & 06322-6-34 & 32 & 1934 & 1.8 & 0.65714 & 7.9 \\
\hline 29 & 06322-6-8 & 270 & 11675 & 0.5 & 0.78784 & 2.5 \\
\hline 29 & $06322-6-43$ & 103 & 4757 & 0.7 & 0.83368 & 2.2 \\
\hline 29 & 06322-6-59 & 319 & 23515 & 1.6 & 1.67078 & 2.3 \\
\hline 29 & $06322-6-30$ & 97 & 3406 & 0.7 & 1.55740 & 4.2 \\
\hline 29 & 06322-6-19 & 189 & 10979 & 0.9 & 1.90089 & 2.9 \\
\hline 29 & $06322-6-42$ & 97 & 11139 & 1.0 & 1.89581 & 4.4 \\
\hline 29 & 06322-6-36 & 653 & 57266 & 1.1 & 2.09162 & 2.4 \\
\hline 29 & $06322-6-33$ & 87 & 11787 & 1.8 & 2.20232 & 2.6 \\
\hline 29 & 06322-6-40 & 85 & 11343 & 0.8 & 2.28935 & 2.4 \\
\hline 29 & 06322-6-17 & 83 & 6311 & 1.1 & 2.33233 & 2.5 \\
\hline 29 & 06322-6-21 & 223 & 21554 & 1.2 & 2.50805 & 2.0 \\
\hline 29 & 06322-6-11 & 87 & 9906 & 0.9 & 2.77320 & 3.5 \\
\hline 29 & $06322-6-56$ & 220 & 16528 & 1.2 & 2.81722 & 1.9 \\
\hline 29 & $06322-6-2$ & 89 & 11177 & 0.6 & 3.03448 & 1.5 \\
\hline 29 & $06322-6-58$ & 220 & 16394 & 0.8 & 2.98128 & 3.1 \\
\hline 29 & 06322-6-1 & 50 & 7095 & 0.5 & 3.14912 & 2.6 \\
\hline 29 & $06322-6-46$ & 207 & 11204 & 1.0 & 3.13993 & 2.3 \\
\hline 29 & 06322-6-35 & 195 & 6238 & 0.4 & 3.21034 & 4.4 \\
\hline 29 & 06322-6-25 & 88 & 4492 & 0.6 & 3.12900 & 2.6 \\
\hline 29 & 06322-6-15 & 492 & 38306 & 6.8 & 3.07640 & 3.6 \\
\hline 29 & $06322-6-22$ & 324 & 12710 & 0.7 & 3.16852 & 3.5 \\
\hline 29 & $06322-6-47$ & 249 & 35318 & 1.9 & 3.41965 & 2.4 \\
\hline 29 & $06322-6-20$ & 1589 & 7015 & 1.6 & 2.85164 & 5.2 \\
\hline 29 & $06322-6-16$ & 564 & 58877 & 2.6 & 3.73880 & 2.2 \\
\hline 29 & $06322-6-54$ & 272 & 28263 & 1.9 & 4.20984 & 5.8 \\
\hline 29 & $06322-6-50$ & 288 & 20022 & 1.2 & 3.97133 & 6.2 \\
\hline 29 & $06322-6-27$ & 155 & 26115 & 0.7 & 4.37582 & 2.3 \\
\hline 29 & $06322-6-60$ & 224 & 19438 & 0.7 & 4.52769 & 4.7 \\
\hline 29 & $06322-6-41$ & 1110 & 42564 & 53.2 & 4.81904 & 4.8 \\
\hline 29 & $06322-6-55$ & 201 & 5637 & 1.0 & 4.60003 & 2.7 \\
\hline 29 & $06322-6-29$ & 190 & 39439 & 0.4 & 4.99345 & 2.0 \\
\hline 29 & 06322-6-5 & 379 & 38323 & 2.5 & 4.92478 & 1.4 \\
\hline 29 & $06322-6-57$ & 464 & 39588 & 0.4 & 5.29624 & 4.2 \\
\hline 29 & $06322-6-12$ & 48 & 6166 & 0.4 & 5.09823 & 2.7 \\
\hline 29 & $06322-6-6$ & 432 & 63019 & 1.4 & 5.07262 & 2.3 \\
\hline 29 & 06322-6-52 & 302 & 43672 & 0.6 & 5.42812 & 1.9 \\
\hline 29 & 06322-6-18 & 231 & 14715 & 3.2 & 5.75418 & 5.2 \\
\hline 29 & $06322-6-3$ & 13 & 3014 & 0.9 & 11.36398 & 6.5 \\
\hline 30 & $06322-37-3$ & 152 & 275 & 0.6 & 0.02234 & 32.3 \\
\hline 30 & $06322-37-29$ & 246 & 639 & 0.3 & 0.03218 & 14.3 \\
\hline 30 & $06322-37-11$ & 265 & 975 & 1.3 & 0.03746 & 7.0 \\
\hline 30 & $06322-37-43$ & 934 & 2862 & 1.2 & 0.03571 & 5.3 \\
\hline 30 & $06322-37-39$ & 164 & 483 & 0.3 & 0.03179 & 13.5 \\
\hline 30 & $06322-37-52$ & 100 & 900 & 1.1 & 0.10341 & 11.8 \\
\hline 30 & $06322-37-28$ & 300 & 2364 & 1.1 & 0.08791 & 4.0 \\
\hline 30 & $06322-37-54$ & 395 & 3925 & 0.3 & 0.10127 & 4.3 \\
\hline 30 & $06322-37-21$ & 249 & 2148 & 0.4 & 0.09882 & 3.5 \\
\hline 30 & $06322-37-14$ & 237 & 2330 & 0.7 & 0.10176 & 4.0 \\
\hline 30 & $06322-37-56$ & 349 & 6325 & 0.2 & 0.16829 & 5.1 \\
\hline 30 & $06322-37-41$ & 225 & 3196 & 0.3 & 0.18220 & 5.0 \\
\hline 30 & $06322-37-37$ & 482 & 6132 & 0.5 & 0.18387 & 1.8 \\
\hline 30 & $06322-37-51$ & 210 & 4177 & 0.6 & 0.19844 & 5.7 \\
\hline 30 & 06322-37-34 & 859 & 8696 & 1.0 & 0.25761 & 4.7 \\
\hline 30 & $06322-37-24$ & 459 & 4001 & 3.7 & 0.49534 & 3.4 \\
\hline 30 & $06322-37-38$ & 317 & 10859 & 0.7 & 0.52639 & 3.0 \\
\hline 30 & $06322-37-13$ & 446 & 15764 & 0.8 & 0.54131 & 2.3 \\
\hline 30 & $06322-37-60$ & 112 & 2112 & 0.4 & 0.66300 & 6.9 \\
\hline 30 & $06322-37-5 a$ & 814 & 29216 & 3.4 & 0.74014 & 2.7 \\
\hline 30 & $06322-37-0$ & 751 & 34181 & 3.1 & 0.75085 & 1.9 \\
\hline 30 & $06322-37-5$ & 67 & 3091 & 0.7 & 0.79677 & 4.3 \\
\hline 30 & 06322-37-46 & 108 & 6465 & 1.3 & 0.81171 & 1.9 \\
\hline 30 & $06322-37-55$ & 35 & 3535 & 0.8 & 0.88351 & 3.6 \\
\hline
\end{tabular}


Kimbrough, D.L., Grove, M., Gehrels, G.E., Dorsey, R.J., Howard, K.A., Lovera, O., Aslan, A., House, P.K., and Pearthree, P.A., 2015, Detrital zircon U-Pb provenance of the Colorado River: A 5 m.y. record of incision into cover strata overlying the Colorado Plateau and adjacent regions: Geosphere, v. 11, doi:10.1130/GES00982.1.

TABLE DR7. U-Pb ZIRCON LA-ICP-MS GEOCHRONOLOGIC ANALYSES OF COLORADO RIVER BASIN \& ASSOCIATED SAMPLES.

\begin{tabular}{|c|c|c|c|c|c|c|}
\hline & & & & & & opic ra \\
\hline ID\# & Analysis & $\begin{array}{c}\text { U } \\
(\mathrm{ppm})\end{array}$ & $\begin{array}{l}206 \mathrm{~Pb} \\
204 \mathrm{~Pb}\end{array}$ & $\mathrm{U} / \mathrm{Th}$ & $\begin{array}{c}207 \mathrm{~Pb}^{*} \\
235 \mathrm{U}\end{array}$ & $\begin{array}{c} \pm \\
(\%) \\
\end{array}$ \\
\hline 30 & $06322-37-2$ & 90 & 7734 & 0.5 & 1.83780 & 2.2 \\
\hline 30 & $06322-37-62$ & 339 & 36721 & 1.2 & 1.76048 & 2.2 \\
\hline 30 & $06322-37-36$ & 119 & 12706 & 0.8 & 1.78563 & 3.3 \\
\hline 30 & 06322-37-4 & 132 & 10391 & 0.9 & 1.95722 & 3.3 \\
\hline 30 & $06322-37-50$ & 124 & 20503 & 0.9 & 1.93689 & 3.2 \\
\hline 30 & $06322-37-64$ & 78 & 10061 & 1.1 & 2.04291 & 2.5 \\
\hline 30 & $06322-37-47$ & 61 & 7897 & 0.5 & 2.07628 & 1.4 \\
\hline 30 & $06322-37-26$ & 588 & 69900 & 7.8 & 2.18742 & 1.6 \\
\hline 30 & $06322-37-65$ & 87 & 11293 & 0.6 & 2.67571 & 3.3 \\
\hline 30 & $06322-37-33$ & 419 & 46623 & 2.1 & 1.99722 & 1.9 \\
\hline 30 & $06322-37-48$ & 186 & 23300 & 0.4 & 3.18805 & 1.4 \\
\hline 30 & $06322-37-23$ & 334 & 36539 & 0.8 & 3.12760 & 2.8 \\
\hline 30 & 06322-37-32 & 171 & 30671 & 1.3 & 3.17851 & 2.5 \\
\hline 30 & $06322-37-30$ & 1156 & 38055 & 1.4 & 3.06016 & 2.7 \\
\hline 30 & $06322-37-31$ & 188 & 29806 & 0.5 & 3.26694 & 2.6 \\
\hline 30 & $06322-37-44$ & 50 & 8564 & 0.3 & 3.57292 & 3.1 \\
\hline 30 & 06322-37-15 & 170 & 26251 & 0.4 & 3.78097 & 2.6 \\
\hline 30 & 06322-37-35 & 85 & 13683 & 0.7 & 4.11986 & 2.4 \\
\hline 30 & $06322-37-59$ & 39 & 7059 & 1.0 & 4.21657 & 3.5 \\
\hline 30 & $06322-37-19$ & 545 & 78247 & 2.0 & 4.19830 & 2.2 \\
\hline 30 & $06322-37-16$ & 75 & 11280 & 0.3 & 4.33850 & 1.6 \\
\hline 30 & $06322-37-6$ & 1218 & 95814 & 3.5 & 4.13942 & 2.0 \\
\hline 30 & $06322-37-58$ & 428 & 90001 & 1.7 & 4.40939 & 5.4 \\
\hline 30 & $06322-37-20$ & 208 & 31285 & 1.1 & 4.37078 & 2.6 \\
\hline 30 & 06322-37-45 & 356 & 50372 & 7.9 & 4.30182 & 2.9 \\
\hline 30 & $06322-37-25$ & 1629 & 31752 & 0.6 & 4.30989 & 2.6 \\
\hline 30 & 06322-37-42 & 47 & 11220 & 0.4 & 4.40182 & 2.9 \\
\hline 30 & $06322-37-17$ & 206 & 16439 & 0.9 & 4.17061 & 2.2 \\
\hline 30 & $06322-37-53$ & 125 & 30254 & 1.0 & 4.74963 & 2.1 \\
\hline 30 & $06322-37-57$ & 197 & 37907 & 1.5 & 4.55422 & 3.1 \\
\hline 30 & 06322-37-1 & 238 & 39518 & 1.2 & 4.89953 & 2.0 \\
\hline 30 & $06322-37-49$ & 539 & 5059 & 0.8 & 3.43874 & 6.3 \\
\hline 30 & $06322-37-22$ & 128 & 14811 & 2.0 & 4.62651 & 1.5 \\
\hline 30 & $06322-37-10$ & 146 & 26455 & 0.7 & 4.98543 & 3.0 \\
\hline 30 & $06322-37-7$ & 252 & 41043 & 1.0 & 5.59534 & 3.5 \\
\hline 30 & $06322-37-63$ & 122 & 19113 & 0.6 & 7.00439 & 2.2 \\
\hline 30 & $06322-37-18$ & 46 & 8043 & 0.5 & 8.08370 & 4.6 \\
\hline 30 & $06322-37-40$ & 531 & 76745 & 1.6 & 12.13516 & 1.9 \\
\hline 30 & $06322-37-27$ & 93 & 29984 & 0.3 & 14.10331 & 2.6 \\
\hline 31 & $325063-40$ & 1010 & 1025 & 0.9 & 0.00944 & 14.4 \\
\hline 31 & $325063-52$ & 362 & 379 & 1.1 & 0.01950 & 27.1 \\
\hline 31 & $325063-46$ & 1058 & 4340 & 1.6 & 0.03748 & 3.7 \\
\hline 31 & $325063-25$ & 253 & 695 & 1.3 & 0.13219 & 11.8 \\
\hline 31 & 325063-11 & 865 & 6274 & 2.1 & 0.08479 & 3.6 \\
\hline 31 & $325063-8$ & 163 & 1643 & 1.1 & 0.09667 & 6.3 \\
\hline 31 & $325063-5$ & 199 & 2230 & 1.1 & 0.09795 & 6.0 \\
\hline 31 & $325063-51$ & 704 & 5439 & 1.0 & 0.11404 & 3.1 \\
\hline 31 & $325063-6$ & 590 & 5812 & 0.7 & 0.11493 & 3.2 \\
\hline 31 & $325063-20$ & 524 & 8354 & 1.2 & 0.17087 & 1.8 \\
\hline 31 & $325063-15$ & 226 & 4403 & 0.8 & 0.19524 & 2.6 \\
\hline 31 & 325063-39 & 230 & 2634 & 0.7 & 0.22166 & 7.0 \\
\hline 31 & $325063-41$ & 324 & 6535 & 2.0 & 0.23254 & 5.7 \\
\hline 31 & $325063-17$ & 266 & 5583 & 0.6 & 0.25354 & 3.1 \\
\hline 31 & $325063-3$ & 127 & 3205 & 1.6 & 0.26228 & 2.1 \\
\hline 31 & $325063-55$ & 185 & 4166 & 0.8 & 0.26951 & 4.0 \\
\hline 31 & $325063-12$ & 88 & 1716 & 0.9 & 0.32116 & 20.0 \\
\hline 31 & 325063-31 & 217 & 5747 & 1.3 & 0.31954 & 3.3 \\
\hline 31 & $325063-53$ & 238 & 7793 & 109.1 & 0.42303 & 2.3 \\
\hline 31 & $325063-28$ & 352 & 19169 & 1.4 & 0.51527 & 1.4 \\
\hline 31 & $325063-43$ & 118 & 10755 & 1.4 & 0.91713 & 3.5 \\
\hline 31 & $325063-27$ & 446 & 40770 & 2.3 & 1.67082 & 1.8 \\
\hline 31 & $325063-50$ & 285 & 34898 & 1.8 & 1.79908 & 1.8 \\
\hline 31 & 325063-32 & 91 & 9882 & 2.6 & 1.83311 & 3.0 \\
\hline 31 & $325063-49$ & 142 & 19140 & 2.8 & 1.99873 & 3.3 \\
\hline 31 & $325063-24$ & 148 & 19693 & 2.0 & 1.81438 & 2.0 \\
\hline 31 & $325063-35$ & 368 & 18132 & 2.8 & 1.97761 & 2.9 \\
\hline 31 & $325063-2$ & 158 & 23132 & 2.4 & 2.16126 & 5.3 \\
\hline 31 & $325063-48$ & 301 & 15330 & 3.8 & 1.99196 & 1.5 \\
\hline 31 & $325063-54$ & 65 & 5887 & 0.7 & 2.04986 & 6.1 \\
\hline 31 & $325063-19$ & 88 & 12355 & 1.5 & 2.32677 & 2.2 \\
\hline 31 & $325063-14$ & 60 & 3644 & 2.3 & 2.17855 & 7.9 \\
\hline 31 & 325063-18 & 397 & 48801 & 1.4 & 2.91671 & 4.2 \\
\hline 31 & 325063-34 & 92 & 15098 & 1.2 & 3.16080 & 1.7 \\
\hline 31 & $325063-44$ & 141 & 26187 & 2.9 & 3.11486 & 2.2 \\
\hline 31 & $325063-60$ & 19 & 2011 & 2.9 & 2.40062 & 4.2 \\
\hline 31 & $325063-33$ & 86 & 9482 & 0.9 & 3.14215 & 2.0 \\
\hline 31 & $325063-23$ & 41 & 7350 & 0.7 & 3.24885 & 2.2 \\
\hline 31 & $325063-9$ & 146 & 24415 & 1.0 & 3.35115 & 1.5 \\
\hline 31 & $325063-4$ & 93 & 3224 & 1.3 & 3.31746 & 2.5 \\
\hline 31 & 325063-38 & 68 & 3428 & 2.2 & 3.41882 & 3.3 \\
\hline 31 & $325063-1$ & 104 & 19858 & 1.1 & 4.02815 & 1.9 \\
\hline 31 & $325063-56$ & 34 & 7049 & 0.6 & 4.36298 & 2.5 \\
\hline 31 & $325063-29$ & 110 & 13822 & 1.7 & 4.15353 & 1.9 \\
\hline 31 & $325063-21$ & 136 & 22677 & 1.1 & 4.12248 & 2.6 \\
\hline 31 & $325063-30$ & 75 & 10930 & 1.4 & 4.25945 & 3.2 \\
\hline 31 & $325063-16$ & 316 & 57730 & 1.8 & 4.27313 & 1.7 \\
\hline 31 & $325063-22$ & 55 & 15055 & 2.1 & 4.20407 & 1.9 \\
\hline 31 & $325063-58$ & 241 & 20256 & 2.8 & 4.40498 & 1.7 \\
\hline 31 & $325063-36$ & 901 & 99180 & 1.4 & 4.47305 & 4.3 \\
\hline 31 & $325063-57$ & 41 & 8667 & 0.9 & 4.32600 & 2.2 \\
\hline
\end{tabular}


Kimbrough, D.L., Grove, M., Gehrels, G.E., Dorsey, R.J., Howard, K.A., Lovera, O., Aslan, A., House, P.K., and Pearthree, P.A., 2015, Detrital zircon U-Pb provenance of the Colorado River: A 5 m.y. record of incision into cover strata overlying the Colorado Plateau and adjacent regions: Geosphere, v. 11, doi:10.1130/GES00982.1.

TABLE DR7. U-Pb ZIRCON LA-ICP-MS GEOCHRONOLOGIC ANALYSES OF COLORADO RIVER BASIN \& ASSOCIATED SAMPLES.

\begin{tabular}{|c|c|c|c|c|c|c|}
\hline & & & & & & opic ra \\
\hline ID\# & Analysis & $\begin{array}{c}U \\
(\mathrm{ppm})\end{array}$ & $\begin{array}{l}206 \mathrm{~Pb} \\
204 \mathrm{~Pb}\end{array}$ & $\mathrm{U} / \mathrm{Th}$ & $\begin{array}{c}207 \mathrm{~Pb}^{*} \\
235 \mathrm{U}\end{array}$ & $\begin{array}{c} \pm \\
(\%) \\
\end{array}$ \\
\hline 31 & $325063-10$ & 388 & 34295 & 2.1 & 4.57760 & 3.3 \\
\hline 31 & $325063-45$ & 169 & 17583 & 1.0 & 4.56954 & 4.0 \\
\hline 31 & $325063-13$ & 379 & 48799 & 2.6 & 4.47360 & 2.8 \\
\hline 31 & $325063-47$ & 94 & 24281 & 1.9 & 4.51598 & 1.4 \\
\hline 31 & $325063-59$ & 260 & 44786 & 2.3 & 4.76112 & 2.0 \\
\hline 31 & $325063-26$ & 121 & 5854 & 1.4 & 4.51458 & 6.3 \\
\hline 31 & $325063-42$ & 306 & 5335 & 3.3 & 4.75349 & 4.1 \\
\hline 31 & 325063-37 & 244 & 52190 & 10.8 & 6.40794 & 2.0 \\
\hline 32 & $1-22-06-2-65$ & 195 & 512 & 1.2 & 0.02584 & 21.7 \\
\hline 32 & $1-22-06-2-9$ & 168 & 355 & 2.4 & 0.02075 & 31.1 \\
\hline 32 & $1-22-06-2-64$ & 143 & 346 & 0.6 & 0.03182 & 27.6 \\
\hline 32 & $1-22-06-2-73$ & 253 & 893 & 1.4 & 0.04276 & 12.7 \\
\hline 32 & $1-22-06-2-19$ & 150 & 573 & 0.5 & 0.05442 & 23.9 \\
\hline 32 & $1-22-06-2-67$ & 316 & 2378 & 0.7 & 0.08140 & 10.3 \\
\hline 32 & $1-22-06-2-62$ & 88 & 853 & 1.3 & 0.10664 & 9.2 \\
\hline 32 & $1-22-06-2-21$ & 429 & 3806 & 2.9 & 0.08197 & 2.9 \\
\hline 32 & $1-22-06-2-63$ & 632 & 5657 & 1.5 & 0.09735 & 11.5 \\
\hline 32 & $1-22-06-2-33$ & 317 & 3602 & 0.5 & 0.09947 & 3.1 \\
\hline 32 & $1-22-06-2-41$ & 167 & 2595 & 0.4 & 0.18915 & 5.5 \\
\hline 32 & $1-22-06-2-24$ & 429 & 6345 & 1.7 & 0.19148 & 7.8 \\
\hline 32 & $1-22-06-2-21$ & 175 & 3086 & 1.1 & 0.17002 & 8.4 \\
\hline 32 & $1-22-06-2-15$ & 459 & 5781 & 3.8 & 0.20213 & 4.3 \\
\hline 32 & $1-22-06-2-2$ & 279 & 6154 & 1.5 & 0.19802 & 5.1 \\
\hline 32 & $1-22-06-2-49$ & 263 & 7402 & 1.3 & 0.19343 & 3.9 \\
\hline 32 & $1-22-06-2-42$ & 106 & 2696 & 5.2 & 0.23902 & 5.0 \\
\hline 32 & $1-22-06-2-59$ & 404 & 10813 & 1.2 & 0.23009 & 3.1 \\
\hline 32 & $1-22-06-2-11$ & 144 & 5044 & 3.5 & 0.26272 & 3.4 \\
\hline 32 & $1-22-06-2-50$ & 119 & 3946 & 1.6 & 0.27104 & 7.1 \\
\hline 32 & $1-22-06-2-50$ & 594 & 13394 & 4.6 & 0.32960 & 2.4 \\
\hline 32 & $1-22-06-2-13$ & 556 & 15950 & 6.2 & 0.32713 & 2.6 \\
\hline 32 & $1-22-06-2-35$ & 208 & 5523 & 2.2 & 0.34263 & 3.0 \\
\hline 32 & $1-22-06-2-29$ & 263 & 6114 & 9.2 & 0.42491 & 3.1 \\
\hline 32 & $1-22-06-2-62$ & 271 & 13257 & 2.4 & 0.50502 & 3.6 \\
\hline 32 & $1-22-06-2-28$ & 336 & 15337 & 1.8 & 0.53419 & 1.6 \\
\hline 32 & $1-22-06-2-52$ & 72 & 4322 & 1.6 & 0.54124 & 6.2 \\
\hline 32 & $1-22-06-2-28$ & 82 & 3713 & 1.6 & 0.62254 & 17.8 \\
\hline 32 & $1-22-06-2-20$ & 52 & 3239 & 4.2 & 0.68978 & 4.3 \\
\hline 32 & $1-22-06-2-6$ & 120 & 8423 & 2.0 & 0.70380 & 3.7 \\
\hline 32 & $1-22-06-2-29$ & 57 & 3482 & 1.6 & 0.73859 & 6.5 \\
\hline 32 & $1-22-06-2-69$ & 183 & 8829 & 1.5 & 0.83891 & 6.1 \\
\hline 32 & $1-22-06-2-45$ & 181 & 14447 & 1.4 & 0.82810 & 2.0 \\
\hline 32 & $1-22-06-2-38$ & 172 & 11261 & 1.6 & 0.81732 & 1.8 \\
\hline 32 & $1-22-06-2-39$ & 87 & 6574 & 1.4 & 0.87308 & 2.4 \\
\hline 32 & $1-22-06-2-37$ & 240 & 22355 & 2.3 & 1.60265 & 4.5 \\
\hline 32 & $1-22-06-2-45$ & 87 & 6728 & 3.4 & 1.62265 & 2.0 \\
\hline 32 & $1-22-06-2-41$ & 172 & 8905 & 2.2 & 1.63598 & 1.6 \\
\hline 32 & $1-22-06-2-3$ & 252 & 35972 & 2.6 & 1.75237 & 2.8 \\
\hline 32 & $1-22-06-2-12$ & 201 & 21959 & 2.8 & 1.69587 & 1.7 \\
\hline 32 & $1-22-06-2-31$ & 202 & 27654 & 7.9 & 1.78682 & 1.9 \\
\hline 32 & $1-22-06-2-59$ & 325 & 31663 & 37.3 & 1.68659 & 2.0 \\
\hline 32 & $1-22-06-2-1$ & 117 & 11404 & 4.2 & 1.84052 & 2.0 \\
\hline 32 & $1-22-06-2-61$ & 160 & 21887 & 3.1 & 1.83673 & 2.0 \\
\hline 32 & $1-22-06-2-47$ & 562 & 20796 & 3.0 & 1.87606 & 1.5 \\
\hline 32 & $1-22-06-2-38$ & 85 & 8777 & 1.7 & 1.96025 & 2.5 \\
\hline 32 & $1-22-06-2-32$ & 190 & 4784 & 2.1 & 1.67853 & 3.1 \\
\hline 32 & $1-22-06-2-17$ & 331 & 36066 & 6.2 & 2.12840 & 3.5 \\
\hline 32 & $1-22-06-2-57$ & 53 & 6116 & 4.1 & 1.89398 & 2.7 \\
\hline 32 & $1-22-06-2-53$ & 291 & 31251 & 4.3 & 1.93140 & 1.8 \\
\hline 32 & $1-22-06-2-20$ & 75 & 4618 & 1.9 & 1.90149 & 6.0 \\
\hline 32 & $1-22-06-2-3$ & 121 & 14716 & 6.0 & 2.08513 & 2.1 \\
\hline 32 & $1-22-06-2-48$ & 63 & 8992 & 4.8 & 1.97703 & 2.3 \\
\hline 32 & $1-22-06-2-39$ & 56 & 3153 & 0.9 & 1.65534 & 7.1 \\
\hline 32 & $1-22-06-2-27$ & 80 & 12140 & 4.2 & 2.01200 & 2.3 \\
\hline 32 & $1-22-06-2-34$ & 120 & 13400 & 3.2 & 2.10623 & 1.8 \\
\hline 32 & $1-22-06-2-23$ & 63 & 10077 & 4.0 & 2.24732 & 4.4 \\
\hline 32 & $1-22-06-2-18$ & 130 & 18995 & 5.7 & 2.03979 & 2.5 \\
\hline 32 & $1-22-06-2-2$ & 90 & 12342 & 5.2 & 2.25640 & 2.0 \\
\hline 32 & $1-22-06-2-43$ & 92 & 11296 & 1.8 & 2.20173 & 2.2 \\
\hline 32 & $1-22-06-2-23$ & 29 & 3989 & 2.0 & 2.21789 & 3.8 \\
\hline 32 & $1-22-06-2-46$ & 192 & 7948 & 1.7 & 2.01474 & 3.5 \\
\hline 32 & $1-22-06-2-51$ & 54 & 10111 & 1.8 & 2.37594 & 2.4 \\
\hline 32 & $1-22-06-2-61$ & 88 & 14422 & 2.2 & 2.44994 & 2.1 \\
\hline 32 & $1-22-06-2-49$ & 187 & 28818 & 2.0 & 2.79092 & 1.7 \\
\hline 32 & $1-22-06-2-55$ & 326 & 41412 & 4.8 & 2.63196 & 9.1 \\
\hline 32 & $1-22-06-2-74$ & 604 & 82515 & 1.5 & 2.75486 & 5.2 \\
\hline 32 & $1-22-06-2-16$ & 95 & 17146 & 1.6 & 2.92310 & 2.3 \\
\hline 32 & $1-22-06-2-10$ & 262 & 16590 & 9.0 & 3.10079 & 6.6 \\
\hline 32 & $1-22-06-2-9$ & 314 & 61258 & 2.4 & 3.15597 & 2.1 \\
\hline 32 & $1-22-06-2-72$ & 49 & 7435 & 0.5 & 2.90866 & 2.1 \\
\hline 32 & $1-22-06-2-65$ & 97 & 12467 & 2.0 & 3.04595 & 2.5 \\
\hline 32 & $1-22-06-2-40$ & 119 & 20122 & 1.2 & 3.20335 & 1.6 \\
\hline 32 & $1-22-06-2-17$ & 111 & 10417 & 2.7 & 3.21412 & 3.6 \\
\hline 32 & $1-22-06-2-70$ & 210 & 38703 & 2.6 & 3.01395 & 3.4 \\
\hline 32 & $1-22-06-2-25$ & 189 & 35340 & 1.3 & 3.27329 & 1.6 \\
\hline 32 & $1-22-06-2-40$ & 255 & 29258 & 2.9 & 3.02965 & 2.2 \\
\hline 32 & $1-22-06-2-4$ & 146 & 19208 & 2.8 & 2.98628 & 3.0 \\
\hline 32 & $1-22-06-2-52$ & 394 & 11584 & 7.4 & 2.96466 & 5.2 \\
\hline 32 & $1-22-06-2-58$ & 81 & 9584 & 1.3 & 3.01038 & 2.8 \\
\hline 32 & $1-22-06-2-66$ & 126 & 25372 & 1.4 & 3.19062 & 1.6 \\
\hline 32 & $1-22-06-2-75$ & 68 & 9041 & 1.0 & 3.13731 & 3.9 \\
\hline
\end{tabular}


Kimbrough, D.L., Grove, M., Gehrels, G.E., Dorsey, R.J., Howard, K.A., Lovera, O., Aslan, A., House, P.K., and Pearthree, P.A., 2015, Detrital zircon U-Pb provenance of the Colorado River: A 5 m.y. record of incision into cover strata overlying the Colorado Plateau and adjacent regions: Geosphere, v. 11, doi:10.1130/GES00982.1.

TABLE DR7. U-Pb ZIRCON LA-ICP-MS GEOCHRONOLOGIC ANALYSES OF COLORADO RIVER BASIN \& ASSOCIATED SAMPLES.

\begin{tabular}{|c|c|c|c|c|c|c|}
\hline & & & & & & opic ra \\
\hline ID\# & Analysis & $\begin{array}{c}\text { U } \\
(\mathrm{ppm})\end{array}$ & $\begin{array}{l}206 \mathrm{~Pb} \\
204 \mathrm{~Pb}\end{array}$ & $\mathrm{U} / \mathrm{Th}$ & $\begin{array}{c}207 \mathrm{~Pb}^{*} \\
235 \mathrm{U}\end{array}$ & $\begin{array}{c} \pm \\
(\%) \\
\end{array}$ \\
\hline 32 & $1-22-06-2-18$ & 28 & 3903 & 0.6 & 2.95729 & 7.5 \\
\hline 32 & $1-22-06-2-44$ & 98 & 16968 & 0.9 & 3.38183 & 1.4 \\
\hline 32 & $1-22-06-2-30$ & 267 & 3147 & 0.8 & 3.44181 & 3.5 \\
\hline 32 & $1-22-06-2-54$ & 83 & 16685 & 4.7 & 3.68812 & 1.7 \\
\hline 32 & $1-22-06-2-51$ & 95 & 25558 & 1.7 & 3.99458 & 1.7 \\
\hline 32 & $1-22-06-2-57$ & 795 & 84655 & 5.5 & 2.97064 & 5.1 \\
\hline 32 & $1-22-06-2-44$ & 77 & 18388 & 1.6 & 4.31936 & 1.6 \\
\hline 32 & $1-22-06-2-11$ & 56 & 11782 & 1.0 & 4.40330 & 2.3 \\
\hline 32 & $1-22-06-2-58$ & 167 & 40124 & 1.8 & 4.34192 & 1.7 \\
\hline 32 & $1-22-06-2-64$ & 42 & 7047 & 1.0 & 4.20145 & 1.9 \\
\hline 32 & $1-22-06-2-12$ & 77 & 19673 & 1.3 & 4.41152 & 1.9 \\
\hline 32 & $1-22-06-2-32$ & 436 & 53177 & 0.8 & 4.56677 & 2.8 \\
\hline 32 & $1-22-06-2-22$ & 123 & 18541 & 2.9 & 4.43503 & 2.3 \\
\hline 32 & $1-22-06-2-48$ & 174 & 13875 & 1.5 & 4.34733 & 4.2 \\
\hline 32 & $1-22-06-2-25$ & 1417 & 54893 & 34.9 & 3.52439 & 9.1 \\
\hline 32 & $1-22-06-2-30$ & 392 & 100315 & 2.4 & 4.26971 & 1.8 \\
\hline 32 & $1-22-06-2-5$ & 137 & 35640 & 1.4 & 4.42605 & 2.0 \\
\hline 32 & $1-22-06-2-16$ & 188 & 40922 & 3.8 & 4.26067 & 1.8 \\
\hline 32 & $1-22-06-2-53$ & 176 & 11056 & 1.9 & 4.36530 & 3.5 \\
\hline 32 & $1-22-06-2-26$ & 195 & 35894 & 3.7 & 4.29979 & 1.5 \\
\hline 32 & $1-22-06-2-22$ & 456 & 10211 & 4.1 & 3.57209 & 5.5 \\
\hline 32 & $1-22-06-2-1$ & 281 & 25157 & 1.9 & 4.58795 & 3.6 \\
\hline 32 & $1-22-06-2-46$ & 420 & 43893 & 8.0 & 4.53479 & 2.0 \\
\hline 32 & $1-22-06-2-63$ & 490 & 114534 & 6.3 & 4.44651 & 1.6 \\
\hline 32 & $1-22-06-2-37$ & 332 & 55160 & 2.0 & 4.58505 & 2.8 \\
\hline 32 & $1-22-06-2-71$ & 266 & 32550 & 2.1 & 4.09588 & 2.1 \\
\hline 32 & $1-22-06-2-14$ & 148 & 14946 & 2.8 & 4.39975 & 2.0 \\
\hline 32 & $1-22-06-2-43$ & 257 & 51198 & 1.4 & 4.45911 & 2.1 \\
\hline 32 & $1-22-06-2-60$ & 406 & 58875 & 1.9 & 4.27272 & 2.4 \\
\hline 32 & $1-22-06-2-8$ & 255 & 30781 & 5.0 & 4.25957 & 2.6 \\
\hline 32 & $1-22-06-2-56$ & 37 & 7223 & 1.6 & 4.75245 & 2.9 \\
\hline 32 & $1-22-06-2-7$ & 39 & 3536 & 2.1 & 4.70625 & 4.5 \\
\hline 32 & $1-22-06-2-55$ & 78 & 19070 & 1.2 & 5.35513 & 1.6 \\
\hline 32 & $1-22-06-2-14$ & 129 & 30600 & 1.5 & 5.59735 & 2.7 \\
\hline 32 & $1-22-06-2-54$ & 59 & 1587 & 1.2 & 5.47715 & 4.9 \\
\hline 32 & $1-22-06-2-19$ & 663 & 8860 & 4.0 & 5.22835 & 4.5 \\
\hline 32 & $1-22-06-2-4$ & 505 & 100912 & 5.0 & 10.87104 & 1.9 \\
\hline 32 & $1-22-06-2-13$ & 46 & 19426 & 1.1 & 13.60094 & 4.1 \\
\hline 32 & $1-22-06-2-24$ & 374 & 110555 & 0.8 & 13.56787 & 1.9 \\
\hline 32 & $1-22-06-2-15$ & 43 & 18178 & 0.8 & 13.80129 & 2.0 \\
\hline 32 & $1-22-06-2-8$ & 73 & 31356 & 1.3 & 13.73076 & 1.8 \\
\hline 33 & $32606-1-31$ & 1403 & 800 & 2.6 & 0.00966 & 10.1 \\
\hline 33 & $32606-1-1-15$ & 2992 & 4254 & 2.6 & 0.02424 & 2.5 \\
\hline 33 & $32606-1-56$ & 51 & 6481 & 0.6 & 2.81125 & 3.2 \\
\hline 33 & $32606-1-47$ & 114 & 12361 & 1.6 & 2.77586 & 3.7 \\
\hline 33 & $32606-1-1-20$ & 43 & 6989 & 0.9 & 2.64968 & 2.7 \\
\hline 33 & $32606-1-45$ & 123 & 6758 & 2.3 & 2.88400 & 1.9 \\
\hline 33 & $32606-1-1-24$ & 1936 & 131725 & 0.9 & 2.72940 & 5.3 \\
\hline 33 & $32606-1-50$ & 54 & 6081 & 0.7 & 2.88256 & 2.5 \\
\hline 33 & $32606-1-44$ & 73 & 11793 & 1.1 & 2.84809 & 2.7 \\
\hline 33 & $32606-1-40$ & 532 & 54523 & 2.2 & 2.81183 & 1.5 \\
\hline 33 & $32606-1-29$ & 105 & 17313 & 1.4 & 2.81925 & 2.6 \\
\hline 33 & $32606-1-54$ & 66 & 5933 & 1.1 & 2.92598 & 3.2 \\
\hline 33 & $32606-1-1-11$ & 87 & 9747 & 1.4 & 2.87121 & 1.4 \\
\hline 33 & $32606-1-1-13$ & 156 & 20729 & 1.8 & 2.90716 & 1.6 \\
\hline 33 & $32606-1-42$ & 187 & 16229 & 0.7 & 2.83300 & 2.6 \\
\hline 33 & $32606-1-1-21$ & 76 & 5710 & 0.7 & 2.73033 & 2.5 \\
\hline 33 & $32606-1-1-4$ & 39 & 4871 & 0.9 & 2.88253 & 2.9 \\
\hline 33 & $32606-1-1-16$ & 4225 & 172849 & 7.1 & 2.76119 & 3.8 \\
\hline 33 & $32606-1-1-9$ & 218 & 19123 & 2.0 & 2.88125 & 2.7 \\
\hline 33 & $32606-1-1-6$ & 1941 & 62340 & 2.3 & 2.77959 & 2.2 \\
\hline 33 & $32606-1-49$ & 528 & 42091 & 1.3 & 2.89994 & 2.0 \\
\hline 33 & $32606-1-1-12$ & 235 & 21396 & 1.8 & 2.85229 & 1.4 \\
\hline 33 & $32606-1-39$ & 178 & 21509 & 1.6 & 2.90762 & 4.4 \\
\hline 33 & $32606-1-58$ & 161 & 28680 & 1.8 & 3.12461 & 2.6 \\
\hline 33 & $32606-1-53$ & 61 & 3611 & 1.4 & 2.88362 & 3.5 \\
\hline 33 & $32606-1-55$ & 156 & 18455 & 4.3 & 3.01256 & 6.3 \\
\hline 33 & $32606-1-37$ & 129 & 5759 & 1.0 & 3.87317 & 2.2 \\
\hline 33 & $32606-1-28$ & 363 & 47034 & 7.2 & 3.74240 & 2.1 \\
\hline 33 & $32606-1-27$ & 712 & 83253 & 8.6 & 4.14121 & 1.8 \\
\hline 33 & $32606-1-51$ & 2312 & 149686 & 13.8 & 4.17890 & 2.7 \\
\hline 33 & $32606-1-34$ & 2372 & 169703 & 15.1 & 4.39772 & 1.8 \\
\hline 33 & $32606-1-25$ & 2071 & 68309 & 7.4 & 3.91098 & 12.2 \\
\hline 33 & $32606-1-33$ & 1373 & 137765 & 40.2 & 4.28000 & 2.3 \\
\hline 33 & $32606-1-36$ & 768 & 59364 & 3.2 & 4.34353 & 3.2 \\
\hline 33 & $32606-1-52$ & 1570 & 129062 & 7.8 & 4.20169 & 1.6 \\
\hline 33 & $32606-1-60$ & 195 & 29736 & 1.0 & 4.64100 & 2.0 \\
\hline 33 & $32606-1-30$ & 681 & 67055 & 1.7 & 4.11262 & 2.4 \\
\hline 33 & $32606-1-1-8$ & 1908 & 173931 & 32.8 & 4.35902 & 1.8 \\
\hline 33 & $32606-1-1-3$ & 1482 & 118983 & 32.3 & 3.94087 & 2.3 \\
\hline 33 & $32606-1-1-22$ & 204 & 35270 & 2.0 & 4.42893 & 3.0 \\
\hline 33 & $32606-1-1-10$ & 370 & 37309 & 1.1 & 4.38491 & 4.3 \\
\hline 33 & $32606-1-2$ & 392 & 40590 & 1.0 & 4.34072 & 1.4 \\
\hline 33 & $32606-1-46$ & 176 & 29978 & 1.0 & 4.43006 & 2.0 \\
\hline 33 & $32606-1-57$ & 2004 & 189921 & 66.1 & 4.44432 & 1.5 \\
\hline 33 & $32606-1-1-18$ & 260 & 29427 & 2.5 & 4.69976 & 4.8 \\
\hline 33 & $32606-1-1-17$ & 427 & 59479 & 28.0 & 4.53794 & 2.6 \\
\hline 33 & $32606-1-26$ & 1642 & 143791 & 2.8 & 4.43184 & 1.5 \\
\hline 33 & $32606-1-43$ & 318 & 55298 & 6.3 & 4.52478 & 1.8 \\
\hline 33 & $32606-1-35$ & 256 & 35940 & 1.6 & 4.30207 & 4.0 \\
\hline
\end{tabular}


Kimbrough, D.L., Grove, M., Gehrels, G.E., Dorsey, R.J., Howard, K.A., Lovera, O., Aslan, A., House, P.K., and Pearthree, P.A., 2015, Detrital zircon U-Pb provenance of the Colorado River: A 5 m.y. record of incision into cover strata overlying the Colorado Plateau and adjacent regions: Geosphere, v. 11, doi:10.1130/GES00982.1.

TABLE DR7. U-Pb ZIRCON LA-ICP-MS GEOCHRONOLOGIC ANALYSES OF COLORADO RIVER BASIN \& ASSOCIATED SAMPLES.

\begin{tabular}{|c|c|c|c|c|c|c|}
\hline & & & & & & topic rat \\
\hline ID\# & Analysis & $\begin{array}{c}\text { U } \\
(\mathrm{ppm})\end{array}$ & $\begin{array}{l}206 \mathrm{~Pb} \\
204 \mathrm{~Pb}\end{array}$ & $\mathrm{U} / \mathrm{Th}$ & $\begin{array}{c}207 \mathrm{~Pb}^{*} \\
235 \mathrm{U}\end{array}$ & $\begin{array}{c} \pm \\
(\%) \\
\end{array}$ \\
\hline 33 & $32606-1-41$ & 84 & 25817 & 2.0 & 5.75237 & 2.6 \\
\hline 33 & $32606-1-1-19$ & 278 & 49485 & 1.6 & 9.88713 & 2.1 \\
\hline 34 & H5HW-21-38 & 938 & 489 & 1.7 & 0.00729 & 13.1 \\
\hline 34 & H5HW-21-24 & 1095 & 1635 & 0.9 & 0.03040 & 5.9 \\
\hline 34 & H5HW-21-34 & 241 & 639 & 1.5 & 0.02600 & 25.4 \\
\hline 34 & H5HW-21-32 & 396 & 619 & 1.4 & 0.02992 & 9.0 \\
\hline 34 & $2 \mathrm{H} 5 \mathrm{HW} 21-13<>$ & 315 & 6102 & 0.8 & 0.0289 & 63.3 \\
\hline 34 & $2 \mathrm{H} 5 \mathrm{HW} 21-18<>$ & 121 & 2094 & 1.1 & 0.0329 & 66.3 \\
\hline 34 & $2 \mathrm{H} 5 \mathrm{HW} 21-82<>$ & 146 & 3116 & 1.5 & 0.0341 & 39.9 \\
\hline 34 & $2 \mathrm{H} 5 \mathrm{HW} 21-1<>$ & 169 & 4992 & 1.2 & 0.0379 & 40.3 \\
\hline 34 & $2 \mathrm{H} 5 \mathrm{HW} 21-97<>$ & 436 & 9250 & 1.9 & 0.0302 & 21.0 \\
\hline 34 & $2 \mathrm{H} 5 \mathrm{HW} 21-98<>$ & 86 & 3020 & 1.3 & 0.1038 & 36.1 \\
\hline 34 & H5HW-21-17 & 269 & 575 & 1.6 & 0.03109 & 22.6 \\
\hline 34 & $2 \mathrm{H} 5 \mathrm{HW} 21-96<>$ & 183 & 8474 & 2.3 & 0.0311 & 50.5 \\
\hline 34 & $2 \mathrm{H} 5 \mathrm{HW} 21-54<>$ & 104 & 4231 & 1.0 & 0.0593 & 121.0 \\
\hline 34 & $\mathrm{H} 5 \mathrm{HW}-21-20$ & 426 & 680 & 2.3 & 0.04550 & 31.6 \\
\hline 34 & $2 \mathrm{H} 5 \mathrm{HW} 21-56<>$ & 249 & 3960 & 1.1 & 0.0325 & 38.1 \\
\hline 34 & $\mathrm{H} 5 \mathrm{HW}-21-5$ & 378 & 651 & 0.9 & 0.04043 & 14.4 \\
\hline 34 & $2 \mathrm{H} 5 \mathrm{HW} 21-21<>$ & 656 & 12943 & 0.9 & 0.0390 & 16.6 \\
\hline 34 & $2 \mathrm{H} 5 \mathrm{HW} 21-63<>$ & 111 & 2333 & 2.6 & 0.1144 & 244.0 \\
\hline 34 & $2 \mathrm{H} 5 \mathrm{HW} 21-2<>$ & 213 & 13141 & 1.7 & 0.0650 & 22.2 \\
\hline 34 & H5HW-21-18 & 741 & 1983 & 2.0 & 0.08914 & 4.8 \\
\hline 34 & $2 \mathrm{H} 5 \mathrm{HW} 21-85<>$ & 197 & 12513 & 1.3 & 0.0805 & 17.9 \\
\hline 34 & $2 \mathrm{H} 5 \mathrm{HW} 21-99<>$ & 307 & 9816 & 1.4 & 0.0777 & 24.0 \\
\hline 34 & H5HW-21-14 & 428 & 4021 & 1.5 & 0.10970 & 3.9 \\
\hline 34 & $2 \mathrm{H} 5 \mathrm{HW} 21-5<>$ & 1247 & 114558 & 5.4 & 0.0991 & 2.7 \\
\hline 34 & $2 \mathrm{H} 5 \mathrm{HW} 21-14<>$ & 53 & 4789 & 2.1 & 0.0755 & 95.7 \\
\hline 34 & $2 \mathrm{H} 5 \mathrm{HW} 21-64<>$ & 210 & 15430 & 1.0 & 0.0953 & 15.2 \\
\hline 34 & H5HW-21-2 & 1807 & 13067 & 1.8 & 0.11046 & 3.2 \\
\hline 34 & H5HW-21-9 & 777 & 8432 & 1.2 & 0.17376 & 2.4 \\
\hline 34 & H5HW-21-44 & 370 & 1441 & 0.9 & 0.22657 & 7.6 \\
\hline 34 & $2 \mathrm{H} 5 \mathrm{HW} 21-9<>$ & 281 & 36829 & 1.3 & 0.1769 & 4.7 \\
\hline 34 & $2 \mathrm{H} 5 \mathrm{HW} 21-92<>$ & 444 & 1645 & 0.9 & 0.1978 & 9.1 \\
\hline 34 & $2 \mathrm{H} 5 \mathrm{HW} 21-30<>$ & 237 & 18456 & 0.4 & 0.1753 & 8.2 \\
\hline 34 & $2 \mathrm{H} 5 \mathrm{HW} 21-3<>$ & 38 & 3274 & 1.5 & 0.1793 & 25.5 \\
\hline 34 & $2 \mathrm{H} 5 \mathrm{HW} 21-77<>$ & 407 & 29159 & 1.5 & 0.2923 & 3.6 \\
\hline 34 & $2 \mathrm{H} 5 \mathrm{HW} 21-59<>$ & 396 & 12373 & 1.7 & 0.4098 & 3.8 \\
\hline 34 & $2 \mathrm{H} 5 \mathrm{HW} 21-76<>$ & 259 & 5929 & 2.5 & 0.4161 & 5.2 \\
\hline 34 & $2 \mathrm{H} 5 \mathrm{HW} 21-19<>$ & 343 & 21928 & 2.0 & 0.4554 & 3.6 \\
\hline 34 & $2 \mathrm{H} 5 \mathrm{HW} 21-46<>$ & 197 & 82211 & 2.4 & 0.6449 & 3.1 \\
\hline 34 & $2 \mathrm{H} 5 \mathrm{H} W 21-28<>$ & 157 & 43960 & 3.3 & 0.6666 & 4.3 \\
\hline 34 & $2 \mathrm{H} 5 \mathrm{HW} 21-34<>$ & 306 & 202259 & 0.8 & 0.7151 & 2.2 \\
\hline 34 & H5HW-21-4 & 785 & 17912 & 2.3 & 0.73241 & 1.9 \\
\hline 34 & $2 \mathrm{H} 5 \mathrm{HW} 21-45<>$ & 365 & 2172 & 0.9 & 0.7690 & 4.7 \\
\hline 34 & $\mathrm{H} 5 \mathrm{HW}-21-36$ & 428 & 18320 & 2.5 & 0.77034 & 2.3 \\
\hline 34 & H5HW-21-59 & 1244 & 29614 & 1.3 & 0.79719 & 1.8 \\
\hline 34 & $2 \mathrm{H} 5 \mathrm{HW} 21-37<>$ & 400 & 18490 & 1.6 & 0.8114 & 1.7 \\
\hline 34 & $2 \mathrm{H} 5 \mathrm{HW} 21-74<>$ & 335 & 92362 & 1.1 & 0.8824 & 1.6 \\
\hline 34 & $\mathrm{H} 5 \mathrm{HW}-21-42$ & 194 & 11391 & 0.6 & 0.89970 & 1.8 \\
\hline 34 & H5HW-21-21 & 71 & 6865 & 2.0 & 1.77769 & 2.9 \\
\hline 34 & $2 \mathrm{H} 5 \mathrm{HW} 21-57<>$ & 164 & 83254 & 5.2 & 1.7260 & 1.7 \\
\hline 34 & $2 \mathrm{H} 5 \mathrm{HW} 21-61<>$ & 95 & 75012 & 1.9 & 1.8516 & 2.0 \\
\hline 34 & $2 \mathrm{H} 5 \mathrm{HW} 21-79<>$ & 201 & 130941 & 2.8 & 1.9227 & 1.2 \\
\hline 34 & $2 \mathrm{H} 5 \mathrm{HW} 21-66<>$ & 214 & 140645 & 1.0 & 2.0761 & 2.2 \\
\hline 34 & H5HW-21-45 & 328 & 21991 & 1.7 & 2.05896 & 1.6 \\
\hline 34 & H5HW-21-26 & 180 & 8598 & 2.1 & 1.89406 & 3.6 \\
\hline 34 & $2 \mathrm{H} 5 \mathrm{HW} 21-43<>$ & 62 & 33385 & 2.0 & 2.1406 & 1.9 \\
\hline 34 & $2 \mathrm{H} 5 \mathrm{HW} 21-47<>$ & 221 & 163712 & 2.8 & 2.0276 & 1.0 \\
\hline 34 & $2 \mathrm{H} 5 \mathrm{HW} 21-15<>$ & 99 & 87485 & 2.3 & 2.0174 & 2.0 \\
\hline 34 & $2 \mathrm{H} 5 \mathrm{HW} 21-24<>$ & 130 & 78318 & 1.6 & 2.1089 & 2.0 \\
\hline 34 & $2 \mathrm{H} 5 \mathrm{HW} 21-90<>$ & 477 & 638731 & 1.6 & 2.1896 & 1.5 \\
\hline 34 & H5HW-21-39 & 83 & 7114 & 3.3 & 2.19177 & 2.8 \\
\hline 34 & $2 \mathrm{H} 5 \mathrm{HW} 21-23<>$ & 138 & 161720 & 2.2 & 2.3725 & 1.4 \\
\hline 34 & $2 \mathrm{H} 5 \mathrm{HW} 21-100<>$ & 193 & 121984 & 2.5 & 2.4448 & 2.1 \\
\hline 34 & $2 \mathrm{H} 5 \mathrm{HW} 21-81<>$ & 245 & 182333 & 2.6 & 2.5188 & 1.4 \\
\hline 34 & H5HW-21-46 & 106 & 13053 & 6.3 & 2.45201 & 2.2 \\
\hline 34 & $2 \mathrm{H} 5 \mathrm{HW} 21-33<>$ & 138 & 133802 & 3.0 & 2.8208 & 1.3 \\
\hline 34 & H5HW-21-54 & 202 & 16379 & 1.2 & 2.80913 & 1.8 \\
\hline 34 & $2 \mathrm{H} 5 \mathrm{HW} 21-55<>$ & 127 & 124650 & 1.6 & 2.9506 & 1.1 \\
\hline 34 & $2 \mathrm{H} 5 \mathrm{HW} 21-50<>$ & 293 & 227251 & 1.1 & 2.8817 & 4.0 \\
\hline 34 & $2 \mathrm{H} 5 \mathrm{HW} 21-95<>$ & 69 & 84377 & 1.0 & 3.0580 & 1.6 \\
\hline 34 & H5HW-21-47 & 221 & 16226 & 2.2 & 2.96066 & 8.8 \\
\hline 34 & $2 \mathrm{H} 5 \mathrm{HW} 21-7<>$ & 719 & 161542 & 0.9 & 3.0875 & 1.4 \\
\hline 34 & H5HW-21-23 & 1173 & 13842 & 2.3 & 2.69833 & 2.2 \\
\hline 34 & $2 \mathrm{H} 5 \mathrm{HW} 21-75<>$ & 425 & 471601 & 3.0 & 3.1190 & 1.6 \\
\hline 34 & $\mathrm{H} 5 \mathrm{HW}-21-49$ & 99 & 10720 & 1.6 & 3.13870 & 1.9 \\
\hline 34 & $2 \mathrm{H} 5 \mathrm{HW} 21-31<>$ & 177 & 219420 & 3.3 & 3.0736 & 1.1 \\
\hline 34 & $2 \mathrm{H} 5 \mathrm{HW} 21-11<>$ & 85 & 111529 & 1.7 & 3.2251 & 2.1 \\
\hline 34 & $2 \mathrm{H} 5 \mathrm{HW} 21-93<>$ & 184 & 219701 & 1.0 & 3.2125 & 0.8 \\
\hline 34 & $2 \mathrm{H} 5 \mathrm{HW} 21-25<>$ & 120 & 45852 & 1.0 & 3.1676 & 1.1 \\
\hline 34 & $2 \mathrm{H} 5 \mathrm{HW} 21-88<>$ & 76 & 19744 & 1.2 & 3.2033 & 3.1 \\
\hline 34 & $2 \mathrm{H} 5 \mathrm{HW} 21-26<>$ & 110 & 80633 & 1.8 & 3.2122 & 1.9 \\
\hline 34 & H5HW-21-57 & 214 & 10423 & 1.7 & 2.89741 & 3.6 \\
\hline 34 & $2 \mathrm{H} 5 \mathrm{HW} 21-38<>$ & 245 & 246584 & 2.4 & 3.1647 & 0.9 \\
\hline 34 & $2 \mathrm{H} 5 \mathrm{HW} 21-68<>$ & 86 & 140906 & 2.7 & 3.3982 & 1.8 \\
\hline 34 & $2 \mathrm{H} 5 \mathrm{HW} 21-32<>$ & 72 & 27052 & 1.1 & 3.2173 & 2.1 \\
\hline 34 & H5HW-21-51 & 166 & 9317 & 1.2 & 2.89386 & 4.6 \\
\hline 34 & H5HW-21-7 & 143 & 16146 & 2.2 & 3.19521 & 1.4 \\
\hline 34 & $2 \mathrm{H} 5 \mathrm{HW} 21-53<>$ & 41 & 68412 & 1.4 & 3.4077 & 3.0 \\
\hline 34 & H5HW-21-52 & 588 & 35568 & 2.3 & 3.16809 & 6.2 \\
\hline
\end{tabular}


Kimbrough, D.L., Grove, M., Gehrels, G.E., Dorsey, R.J., Howard, K.A., Lovera, O., Aslan, A., House, P.K., and Pearthree, P.A., 2015, Detrital zircon U-Pb provenance of the Colorado River: A 5 m.y. record of incision into cover strata overlying the Colorado Plateau and adjacent regions: Geosphere, v. 11, doi:10.1130/GES00982.1.

TABLE DR7. U-Pb ZIRCON LA-ICP-MS GEOCHRONOLOGIC ANALYSES OF COLORADO RIVER BASIN \& ASSOCIATED SAMPLES.

\begin{tabular}{|c|c|c|c|c|c|c|}
\hline & & & & & & opic rat \\
\hline ID\# & Analysis & $\begin{array}{c}\text { U } \\
(\mathrm{ppm})\end{array}$ & $\begin{array}{l}206 \mathrm{~Pb} \\
204 \mathrm{~Pb} \\
\end{array}$ & U/Th & $\begin{array}{c}207 \mathrm{~Pb}^{*} \\
235 \mathrm{U}\end{array}$ & $\begin{array}{c} \pm \\
(\%) \\
\end{array}$ \\
\hline 34 & H5HW-21-33 & 239 & 27364 & 1.8 & 3.81892 & 3.3 \\
\hline 34 & H5HW-21-16 & 139 & 17674 & 2.9 & 4.05138 & 2.6 \\
\hline 34 & H5HW-21-53 & 890 & 50539 & 2.3 & 3.62721 & 5.7 \\
\hline 34 & H5HW-21-12 & 150 & 19734 & 2.3 & 4.07818 & 1.7 \\
\hline 34 & $2 \mathrm{H} 5 \mathrm{HW} 21-41<>$ & 133 & 159735 & 2.0 & 4.3376 & 1.0 \\
\hline 34 & H5HW-21-22 & 495 & 20761 & 2.1 & 2.21714 & 7.1 \\
\hline 34 & $2 \mathrm{H} 5 \mathrm{HW} 21-71<>$ & 76 & 65492 & 1.6 & 4.2941 & 1.5 \\
\hline 34 & H5HW-21-60 & 153 & 5537 & 1.1 & 3.60642 & 6.6 \\
\hline 34 & H5HW-21-56 & 82 & 5319 & 1.4 & 4.01058 & 4.5 \\
\hline 34 & $\mathrm{H} 5 \mathrm{HW}-21-30$ & 878 & 16164 & 1.2 & 3.42020 & 5.0 \\
\hline 34 & $2 \mathrm{H} 5 \mathrm{HW} 21-80<>$ & 166 & 177500 & 3.2 & 4.4280 & 2.6 \\
\hline 34 & H5HW-21-48 & 214 & 11102 & 1.7 & 4.10367 & 2.1 \\
\hline 34 & $2 \mathrm{H} 5 \mathrm{HW} 21-72<>$ & 380 & 21425 & 2.3 & 4.4709 & 1.2 \\
\hline 34 & H5HW-21-41 & 253 & 34582 & 2.0 & 4.25165 & 2.9 \\
\hline 34 & $2 \mathrm{H} 5 \mathrm{HW} 21-20<>$ & 95 & 103208 & 1.4 & 4.3246 & 1.2 \\
\hline 34 & $2 \mathrm{H} 5 \mathrm{HW} 21-94<>$ & 109 & 206536 & 1.6 & 4.5921 & 1.4 \\
\hline 34 & $2 \mathrm{H} 5 \mathrm{HW} 21-62<>$ & 351 & 41195 & 2.2 & 4.3341 & 1.7 \\
\hline 34 & $2 \mathrm{H} 5 \mathrm{HW} 21-22<>$ & 259 & 158660 & 4.0 & 4.3544 & 1.3 \\
\hline 34 & $2 \mathrm{H} 5 \mathrm{HW} 21-91<>$ & 360 & 82615 & 1.6 & 4.5229 & 2.9 \\
\hline 34 & $2 \mathrm{H} 5 \mathrm{HW} 21-29<>$ & 740 & 67195 & 23.7 & 4.3892 & 3.9 \\
\hline 34 & $2 \mathrm{H} 5 \mathrm{HW} 21-10<>$ & 107 & 129850 & 1.6 & 4.3121 & 1.5 \\
\hline 34 & $2 \mathrm{H} 5 \mathrm{HW} 21-89<>$ & 177 & 193093 & 1.7 & 4.5129 & 1.2 \\
\hline 34 & $2 \mathrm{H} 5 \mathrm{HW} 21-84<>$ & 132 & 158397 & 1.9 & 4.5720 & 0.9 \\
\hline 34 & $2 \mathrm{H} 5 \mathrm{HW} 21-83<>$ & 310 & 12740 & 0.9 & 4.0403 & 2.5 \\
\hline 34 & H5HW-21-35 & 153 & 19440 & 1.3 & 4.33759 & 1.6 \\
\hline 34 & H5HW-21-8 & 219 & 14136 & 1.5 & 4.11298 & 3.4 \\
\hline 34 & $\mathrm{H} 5 \mathrm{HW}-21-50$ & 166 & 20434 & 2.1 & 4.45142 & 2.5 \\
\hline 34 & $2 \mathrm{H} 5 \mathrm{HW} 21-58<>$ & 248 & 368566 & 2.5 & 4.5146 & 2.1 \\
\hline 34 & H5HW-21-58 & 341 & 6556 & 1.7 & 4.31667 & 2.7 \\
\hline 34 & H5HW-21-15 & 350 & 2285 & 2.5 & 3.94984 & 3.6 \\
\hline 34 & $2 \mathrm{H} 5 \mathrm{HW} 21-67<>$ & 179 & 274124 & 2.0 & 4.6000 & 0.9 \\
\hline 34 & $2 \mathrm{H} 5 \mathrm{HW} 21-51<>$ & 282 & 607719 & 3.9 & 4.5395 & 0.9 \\
\hline 34 & 2H5HW21-6 <> & 244 & 304873 & 1.6 & 4.6768 & 1.4 \\
\hline 34 & $2 \mathrm{H} 5 \mathrm{HW} 21-36<>$ & 196 & 22384 & 2.1 & 4.1516 & 4.5 \\
\hline 34 & H5HW-21-25 & 181 & 5197 & 1.6 & 4.52628 & 4.0 \\
\hline 34 & $2 \mathrm{H} 5 \mathrm{HW} 21-39<>$ & 110 & 99726 & 2.3 & 4.8586 & 3.3 \\
\hline 34 & H5HW-21-29 & 450 & 12840 & 2.1 & 4.33179 & 4.6 \\
\hline 34 & H5HW-21-27 & 493 & 64504 & 1.6 & 4.82463 & 3.1 \\
\hline 34 & H5HW-21-10 & 287 & 28544 & 1.8 & 4.52592 & 2.3 \\
\hline 34 & $2 \mathrm{H} 5 \mathrm{HW} 21-60<>$ & 51 & 112899 & 0.8 & 4.9501 & 1.8 \\
\hline 34 & H5HW-21-13 & 962 & 119521 & 3.9 & 4.39183 & 2.0 \\
\hline 34 & $2 \mathrm{H} 5 \mathrm{HW} 21-44<>$ & 299 & 398843 & 3.3 & 4.9864 & 1.1 \\
\hline 34 & $2 \mathrm{H} 5 \mathrm{HW} 21-40<>$ & 147 & 20238 & 2.5 & 4.8313 & 3.0 \\
\hline 34 & H5HW-21-28 & 398 & 44471 & 2.2 & 4.96460 & 3.5 \\
\hline 34 & H5HW-21-21-1 & 286 & 43108 & 3.2 & 5.60706 & 2.4 \\
\hline 34 & H5HW-21-43 & 247 & 53504 & 2.0 & 5.95427 & 1.8 \\
\hline 34 & $2 \mathrm{H} 5 \mathrm{HW} 21-49<>$ & 50 & 67121 & 2.3 & 7.4990 & 1.2 \\
\hline 34 & H5HW-21-55 & 111 & 19203 & 1.5 & 12.27246 & 1.8 \\
\hline 34 & H5HW-21-19 & 164 & 39146 & 2.1 & 12.54911 & 4.6 \\
\hline 34 & H5HW-21-6 & 160 & 37536 & 0.8 & 13.26835 & 1.5 \\
\hline 34 & H5HW-21-37 & 100 & 28510 & 19.7 & 18.04300 & 10.1 \\
\hline 35 & LMSP2-18 & 416 & 899 & 1.2 & 0.02991 & 12.7 \\
\hline 35 & LMSP2-12 & 384 & 773 & 1.2 & 0.03225 & 19.0 \\
\hline 35 & 2LMSP-2-5 <> & 135 & 1453 & 0.8 & 0.0313 & 47.1 \\
\hline 35 & 2LMSP-2-26 <> & 111 & 1247 & 1.2 & 0.0430 & 151.2 \\
\hline 35 & 2LMSP-2-65 <> & 254 & 4496 & 1.6 & 0.0303 & 36.2 \\
\hline 35 & 2LMSP-2-8 <> & 211 & 849 & 1.0 & 0.0182 & 42.4 \\
\hline 35 & LMSP2-55 & 199 & 401 & 0.7 & 0.02951 & 26.4 \\
\hline 35 & LMSP2-30 & 320 & 1020 & 1.6 & 0.03509 & 14.1 \\
\hline 35 & 2LMSP-2-21 <> & 344 & 7616 & 0.9 & 0.0293 & 31.6 \\
\hline 35 & LMSP2-3 & 833 & 1626 & 0.7 & 0.03547 & 25.9 \\
\hline 35 & LMSP2-39 & 414 & 1161 & 1.9 & 0.03461 & 15.4 \\
\hline 35 & 2LMSP-2-61 <> & 284 & 6656 & 0.7 & 0.0343 & 31.2 \\
\hline 35 & 2LMSP-2-15 <> & 327 & 13083 & 1.1 & 0.0396 & 19.1 \\
\hline 35 & LMSP2-48 & 550 & 1732 & 2.4 & 0.04305 & 9.4 \\
\hline 35 & 2LMSP-2-63 <> & 516 & 4903 & 1.0 & 0.0381 & 14.5 \\
\hline 35 & 2LMSP-2-6 <> & 511 & 9319 & 1.3 & 0.0422 & 13.1 \\
\hline 35 & LMSP2-62 & 632 & 2013 & 1.9 & 0.04987 & 8.2 \\
\hline 35 & LMSP2-60 & 289 & 629 & 2.3 & 0.07857 & 11.1 \\
\hline 35 & LMSP2-27 & 898 & 4972 & 2.4 & 0.05496 & 4.6 \\
\hline 35 & 2LMSP-2-31 <> & 55 & 2229 & 1.4 & 0.1308 & 372.1 \\
\hline 35 & LMSP2-37 & 326 & 1991 & 1.5 & 0.07952 & 5.8 \\
\hline 35 & 2LMSP-2-68 <> & 210 & 13210 & 2.2 & 0.0894 & 14.3 \\
\hline 35 & LMSP2-10 & 584 & 6567 & 2.0 & 0.11970 & 4.7 \\
\hline 35 & 2LMSP-2-72 <> & 81 & 6499 & 1.6 & 0.1329 & 19.8 \\
\hline 35 & 2LMSP-2-17 <> & 267 & 37216 & 0.6 & 0.1640 & 6.2 \\
\hline 35 & 2LMSP-2-69 <> & 77 & 2984 & 0.8 & 0.1538 & 24.2 \\
\hline 35 & 2LMSP-2-75 <> & 333 & 21044 & 1.6 & 0.1825 & 7.4 \\
\hline 35 & 2LMSP-2-2 <> & 189 & 23384 & 0.7 & 0.1830 & 7.8 \\
\hline 35 & 2LMSP-2-32 <> & 554 & 42942 & 1.7 & 0.1839 & 3.1 \\
\hline 35 & LMSP2-56 & 378 & 4060 & 1.9 & 0.21367 & 3.4 \\
\hline 35 & 2LMSP-2-4 <> & 185 & 28908 & 0.7 & 0.2216 & 5.9 \\
\hline 35 & LMSP2-61 & 348 & 8146 & 3.0 & 0.25867 & 2.4 \\
\hline 35 & 2LMSP-2-27 <> & 360 & 48497 & 1.7 & 0.2465 & 5.1 \\
\hline 35 & 2LMSP-2-13 <> & 78 & 14618 & 1.1 & 0.2287 & 15.5 \\
\hline 35 & 2LMSP-2-57 <> & 306 & 44682 & 1.5 & 0.2650 & 7.4 \\
\hline 35 & LMSP2-13 & 79 & 2361 & 1.2 & 0.29067 & 5.9 \\
\hline 35 & 2LMSP-2-16 <> & 325 & 71975 & 1.5 & 0.3088 & 3.6 \\
\hline 35 & LMSP2-14 & 344 & 10173 & 1.1 & 0.38707 & 3.4 \\
\hline 35 & LMSP2-22 & 136 & 5537 & 1.5 & 0.48708 & 3.4 \\
\hline
\end{tabular}


Kimbrough, D.L., Grove, M., Gehrels, G.E., Dorsey, R.J., Howard, K.A., Lovera, O., Aslan, A., House, P.K., and Pearthree, P.A., 2015, Detrital zircon U-Pb provenance of the Colorado River: A 5 m.y. record of incision into cover strata overlying the Colorado Plateau and adjacent regions: Geosphere, v. 11, doi:10.1130/GES00982.1.

TABLE DR7. U-Pb ZIRCON LA-ICP-MS GEOCHRONOLOGIC ANALYSES OF COLORADO RIVER BASIN \& ASSOCIATED SAMPLES.

\begin{tabular}{|c|c|c|c|c|c|c|}
\hline & & & & & & opic ra \\
\hline ID\# & Analysis & $\begin{array}{c}\text { U } \\
(\mathrm{ppm})\end{array}$ & $\begin{array}{l}206 \mathrm{~Pb} \\
204 \mathrm{~Pb}\end{array}$ & $\mathrm{U} / \mathrm{Th}$ & $\begin{array}{c}207 \mathrm{~Pb}^{*} \\
235 \mathrm{U}\end{array}$ & $\begin{array}{c} \pm \\
(\%)\end{array}$ \\
\hline 35 & LMSP2-53 & 50 & 1404 & 2.2 & 0.62802 & 4.8 \\
\hline 35 & 2LMSP-2-43 $<>$ & 180 & 135736 & 1.4 & 0.5180 & 3.4 \\
\hline 35 & 2LMSP-2-46 <> & 142 & 39579 & 1.5 & 0.6503 & 4.6 \\
\hline 35 & LMSP2-51 & 125 & 6651 & 1.2 & 0.80964 & 2.4 \\
\hline 35 & LMSP2-32 & 256 & 14263 & 1.5 & 0.82645 & 2.1 \\
\hline 35 & 2LMSP-2-85 <> & 356 & 189099 & 2.8 & 1.3400 & 1.4 \\
\hline 35 & 2LMSP-2-24 <> & 25 & 17388 & 0.7 & 1.7353 & 6.2 \\
\hline 35 & 2LMSP-2-36 <> & 100 & 84063 & 2.4 & 1.6622 & 2.2 \\
\hline 35 & LMSP2-40 & 56 & 4801 & 1.9 & 1.69082 & 2.4 \\
\hline 35 & 2LMSP-2-81 <> & 133 & 62920 & 3.0 & 1.7968 & 2.9 \\
\hline 35 & LMSP2-15 & 113 & 11874 & 0.7 & 1.73647 & 1.8 \\
\hline 35 & 2LMSP-2-48 <> & 1469 & 396187 & 2.7 & 1.7076 & 1.1 \\
\hline 35 & 2LMSP-2-82 <> & 106 & 141260 & 2.4 & 1.7755 & 1.8 \\
\hline 35 & LMSP2-52 & 581 & 31511 & 7.2 & 1.64597 & 3.2 \\
\hline 35 & LMSP2-9 & 58 & 6422 & 0.8 & 1.88645 & 2.7 \\
\hline 35 & LMSP2-50 & 165 & 14393 & 1.7 & 1.83303 & 3.5 \\
\hline 35 & 2LMSP-2-78 <> & 85 & 25449 & 1.6 & 1.8835 & 2.9 \\
\hline 35 & 2 LMSP-2-25 <> & 176 & 119327 & 1.7 & 1.7529 & 1.2 \\
\hline 35 & 2LMSP-2-80 <> & 207 & 6304 & 0.7 & 1.8185 & 1.9 \\
\hline 35 & LMSP2-43 & 265 & 24590 & 2.1 & 1.91926 & 2.7 \\
\hline 35 & 2LMSP-2-83 <> & 51 & 37276 & 1.5 & 1.8330 & 4.2 \\
\hline 35 & 2LMSP-2-1 <> & 122 & 14059 & 2.0 & 1.8440 & 2.9 \\
\hline 35 & 2LMSP-2-67 <> & 201 & 95012 & 0.8 & 1.9640 & 1.8 \\
\hline 35 & 2LMSP-2-62 <> & 55 & 34792 & 1.6 & 1.9086 & 2.5 \\
\hline 35 & 2LMSP-2-59 <> & 58 & 34414 & 2.3 & 2.1034 & 5.0 \\
\hline 35 & 2 LMSP-2-29 <> & 36 & 23311 & 1.0 & 2.1127 & 5.3 \\
\hline 35 & LMSP2-42 & 248 & 13242 & 1.9 & 2.08331 & 2.3 \\
\hline 35 & LMSP2-7 & 54 & 5338 & 2.6 & 1.77211 & 3.7 \\
\hline 35 & LMSP2-11 & 276 & 29466 & 2.2 & 2.19376 & 2.2 \\
\hline 35 & 2LMSP-2-53 <> & 86 & 116052 & 1.9 & 2.2274 & 1.8 \\
\hline 35 & 2LMSP-2-19 <> & 394 & 254866 & 2.6 & 2.2240 & 0.9 \\
\hline 35 & 2LMSP-2-38 <> & 107 & 59330 & 1.1 & 2.2929 & 1.6 \\
\hline 35 & 2LMSP-2-70 <> & 35 & 22715 & 0.8 & 2.4894 & 4.2 \\
\hline 35 & 2LMSP-2-14 <> & 100 & 58940 & 2.0 & 2.6543 & 1.8 \\
\hline 35 & 2LMSP-2-60 <> & 59 & 152150 & 1.1 & 2.8695 & 2.9 \\
\hline 35 & 2LMSP-2-76 <> & 90 & 124441 & 1.0 & 2.8634 & 2.3 \\
\hline 35 & 2LMSP-2-23 <> & 250 & 10553 & 1.1 & 2.6283 & 2.9 \\
\hline 35 & LMSP2-49 & 135 & 15231 & 1.8 & 2.93234 & 1.8 \\
\hline 35 & LMSP2-58 & 440 & 15654 & 2.3 & 2.79480 & 2.8 \\
\hline 35 & 2LMSP-2-47 <> & 120 & 143646 & 1.6 & 2.9419 & 1.7 \\
\hline 35 & LMSP2-23 & 107 & 17922 & 1.2 & 2.96423 & 1.8 \\
\hline 35 & LMSP2-19 & 137 & 22735 & 1.4 & 2.86315 & 2.2 \\
\hline 35 & LMSP2-17 & 226 & 32047 & 0.9 & 3.05934 & 2.0 \\
\hline 35 & 2LMSP-2-41 <> & 321 & 339915 & 2.2 & 2.9760 & 1.2 \\
\hline 35 & 2 LMSP-2-51 <> & 199 & 83089 & 1.3 & 3.0559 & 1.6 \\
\hline 35 & LMSP2-4 & 223 & 27117 & 2.5 & 3.03215 & 1.5 \\
\hline 35 & LMSP2-16 & 67 & 1950 & 2.6 & 2.24970 & 3.4 \\
\hline 35 & 2LMSP-2-71 <> & 294 & 311861 & 4.2 & 3.0732 & 2.3 \\
\hline 35 & 2 LMSP-2-11 <> & 385 & 38166 & 0.4 & 3.0078 & 0.8 \\
\hline 35 & 2LMSP-2-37 <> & 59 & 56532 & 1.0 & 3.0660 & 2.4 \\
\hline 35 & 2LMSP-2-20 <> & 103 & 115004 & 0.5 & 2.9231 & 1.1 \\
\hline 35 & LMSP2-25 & 100 & 10306 & 1.1 & 3.15603 & 2.3 \\
\hline 35 & 2 LMSP-2-34 <> & 212 & 5163 & 2.2 & 3.0721 & 1.9 \\
\hline 35 & 2LMSP-2-30 <> & 238 & 163005 & 0.4 & 3.1654 & 1.0 \\
\hline 35 & 2LMSP-2-28 <> & 130 & 140526 & 1.1 & 3.1187 & 1.2 \\
\hline 35 & 2LMSP-2-10 <> & 289 & 314364 & 1.2 & 3.1600 & 1.7 \\
\hline 35 & LMSP2-57 & 300 & 35899 & 2.8 & 3.14941 & 1.4 \\
\hline 35 & 2LMSP-2-54 <> & 65 & 114578 & 1.7 & 3.3652 & 2.3 \\
\hline 35 & LMSP2-65 & 36 & 3674 & 1.8 & 3.14395 & 3.9 \\
\hline 35 & LMSP2-28 & 505 & 69916 & 2.0 & 3.53918 & 1.4 \\
\hline 35 & LMSP2-54 & 139 & 17909 & 1.7 & 3.92889 & 3.6 \\
\hline 35 & LMSP2-47 & 140 & 23306 & 2.2 & 3.97962 & 1.9 \\
\hline 35 & LMSP2-8 & 203 & 28379 & 1.4 & 3.86068 & 1.7 \\
\hline 35 & 2LMSP-2-77 <> & 98 & 233655 & 1.2 & 4.1667 & 1.0 \\
\hline 35 & LMSP2-21 & 470 & 23743 & 3.3 & 3.29348 & 10.7 \\
\hline 35 & 2LMSP-2-58 <> & 124 & 98610 & 1.8 & 4.3317 & 1.7 \\
\hline 35 & LMSP2-35 & 338 & 54583 & 2.2 & 4.14829 & 2.3 \\
\hline 35 & LMSP2-33 & 208 & 34086 & 1.2 & 4.23198 & 2.7 \\
\hline 35 & 2 LMSP-2-52 <> & 80 & 100783 & 0.7 & 4.2486 & 2.0 \\
\hline 35 & 2LMSP-2-42 <> & 245 & 117913 & 0.9 & 4.2324 & 1.4 \\
\hline 35 & 2LMSP-2-79 <> & 133 & 206667 & 1.3 & 4.1912 & 1.3 \\
\hline 35 & 2LMSP-2-56 <> & 492 & 679894 & 17.0 & 4.2636 & 0.9 \\
\hline 35 & LMSP2-36 & 86 & 14850 & 1.5 & 4.28180 & 2.1 \\
\hline 35 & 2LMSP-2-50 <> & 76 & 49848 & 2.2 & 3.8473 & 1.6 \\
\hline 35 & LMSP2-29 & 66 & 14019 & 3.1 & 4.34064 & 4.1 \\
\hline 35 & LMSP2-45 & 261 & 27990 & 1.6 & 4.19717 & 2.2 \\
\hline 35 & LMSP2-63 & 34 & 5092 & 1.0 & 4.26387 & 2.3 \\
\hline 35 & LMSP2-31 & 257 & 47246 & 1.8 & 4.22915 & 2.3 \\
\hline 35 & LMSP2-64 & 483 & 47158 & 1.0 & 4.53609 & 3.1 \\
\hline 35 & 2LMSP-2-7 $<>$ & 1940 & 1085685 & 33.3 & 4.2903 & 1.6 \\
\hline 35 & 2LMSP-2-9 $<>$ & 57 & 59651 & 1.5 & 4.3931 & 5.2 \\
\hline 35 & LMSP2-26 & 451 & 78598 & 0.9 & 4.35418 & 1.4 \\
\hline 35 & LMSP2-44 & 473 & 37636 & 6.3 & 4.31619 & 1.8 \\
\hline 35 & 2LMSP-2-49 <> & 131 & 187703 & 1.5 & 4.4511 & 3.0 \\
\hline 35 & 2LMSP-2-35 <> & 108 & 91459 & 2.4 & 4.3419 & 1.9 \\
\hline 35 & 2 LMSP-2-64 <> & 174 & 348703 & 3.4 & 4.5154 & 2.0 \\
\hline 35 & LMSP2-2 & 127 & 25036 & 0.7 & 4.29057 & 2.2 \\
\hline 35 & LMSP2-38 & 322 & 35266 & 1.2 & 4.48213 & 1.6 \\
\hline 35 & 2LMSP-2-39 <> & 395 & 134003 & 1.9 & 4.3051 & 1.7 \\
\hline 35 & 2LMSP-2-33 <> & 235 & 114601 & 1.1 & 4.4513 & 0.7 \\
\hline
\end{tabular}


Kimbrough, D.L., Grove, M., Gehrels, G.E., Dorsey, R.J., Howard, K.A., Lovera, O., Aslan, A., House, P.K., and Pearthree, P.A., 2015, Detrital zircon U-Pb provenance of the Colorado River: A 5 m.y. record of incision into cover strata overlying the Colorado Plateau and adjacent regions: Geosphere, v. 11, doi:10.1130/GES00982.1.

TABLE DR7. U-Pb ZIRCON LA-ICP-MS GEOCHRONOLOGIC ANALYSES OF COLORADO RIVER BASIN \& ASSOCIATED SAMPLES.

\begin{tabular}{|c|c|c|c|c|c|c|}
\hline & & & & & & opic ra \\
\hline ID\# & Analysis & $\begin{array}{c}\text { U } \\
(\mathrm{ppm})\end{array}$ & $\begin{array}{l}206 \mathrm{~Pb} \\
204 \mathrm{~Pb}\end{array}$ & $\mathrm{U} / \mathrm{Th}$ & $\begin{array}{c}207 \mathrm{~Pb}^{*} \\
235 \mathrm{U}\end{array}$ & $\begin{array}{c} \pm \\
(\%) \\
\end{array}$ \\
\hline 35 & LMSP2-34 & 458 & 71976 & 2.7 & 4.49721 & 2.1 \\
\hline 35 & 2LMSP-2-3 $<>$ & 240 & 313221 & 1.4 & 4.4132 & 1.4 \\
\hline 35 & LMSP2-24 & 226 & 38633 & 3.7 & 4.40617 & 2.1 \\
\hline 35 & 2LMSP-2-18 <> & 205 & 83006 & 1.6 & 4.0986 & 2.1 \\
\hline 35 & 2LMSP-2-22 <> & 150 & 118818 & 1.9 & 4.4602 & 1.0 \\
\hline 35 & LMSP2-46 & 51 & 10295 & 1.0 & 5.02834 & 1.6 \\
\hline 35 & 2LMSP-2-45 <> & 103 & 116436 & 3.0 & 5.2530 & 1.6 \\
\hline 35 & 2LMSP-2-66 <> & 118 & 181218 & 1.5 & 5.5750 & 1.4 \\
\hline 35 & LMSP2-5 & 437 & 18682 & 1.4 & 5.97273 & 5.7 \\
\hline 35 & LMSP2-41 & 96 & 11470 & 0.9 & 6.51349 & 2.2 \\
\hline 35 & 2LMSP-2-73 <> & 128 & 628329 & 2.2 & 10.3627 & 1.9 \\
\hline 35 & 2LMSP-2-40 <> & 48 & 115067 & 0.7 & 13.3574 & 2.0 \\
\hline 35 & 2LMSP-2-44 <> & 562 & 1203887 & 1.3 & 14.2078 & 1.4 \\
\hline 35 & LMSP2-59 & 89 & 28933 & 1.7 & 15.99577 & 1.6 \\
\hline 36 & DM305-33 & 1481 & 1140 & 0.9 & 0.06177 & 7.9 \\
\hline 36 & DM305-23 & 382 & 6157 & 1.8 & 0.19326 & 2.9 \\
\hline 36 & DM305-19 & 372 & 3717 & 2.2 & 0.23035 & 12.0 \\
\hline 36 & DM305-59 & 133 & 2031 & 1.5 & 0.32283 & 7.2 \\
\hline 36 & DM305-51 & 334 & 5253 & 4.2 & 0.55863 & 7.1 \\
\hline 36 & DM305-39 & 186 & 3639 & 1.5 & 0.58404 & 3.0 \\
\hline 36 & DM305-31 & 31 & 1300 & 1.4 & 0.85281 & 8.1 \\
\hline 36 & DM305-44 & 130 & 1808 & 1.1 & 0.96860 & 5.5 \\
\hline 36 & DM305-52 & 660 & 45366 & 47.7 & 1.84875 & 1.9 \\
\hline 36 & DM305-32 & 218 & 6067 & 1.7 & 1.81330 & 3.5 \\
\hline 36 & DM305-27 & 219 & 6508 & 3.0 & 1.55985 & 4.9 \\
\hline 36 & DM305-45 & 132 & 7892 & 21.4 & 1.93214 & 6.9 \\
\hline 36 & DM305-54 & 369 & 20163 & 2.2 & 2.15459 & 2.3 \\
\hline 36 & DM305-40 & 451 & 16514 & 4.2 & 2.10878 & 2.4 \\
\hline 36 & DM305-60 & 46 & 6967 & 2.0 & 1.98210 & 2.7 \\
\hline 36 & DM305-34 & 271 & 15880 & 2.1 & 2.66106 & 2.1 \\
\hline 36 & DM305-37 & 3030 & 142563 & 12.5 & 2.77932 & 3.3 \\
\hline 36 & DM305-17 & 89 & 11077 & 0.9 & 2.78423 & 1.8 \\
\hline 36 & DM305-16 & 88 & 8511 & 1.4 & 2.78041 & 2.8 \\
\hline 36 & DM305-53 & 96 & 11016 & 1.6 & 2.77851 & 2.2 \\
\hline 36 & DM305-41 & 62 & 7789 & 1.3 & 2.88572 & 2.0 \\
\hline 36 & DM305-43 & 185 & 20215 & 2.8 & 2.89334 & 2.1 \\
\hline 36 & DM305-5 & 1488 & 69171 & 3.2 & 2.66335 & 4.0 \\
\hline 36 & DM305-48 & 129 & 11622 & 1.9 & 2.71977 & 2.5 \\
\hline 36 & DM305-21 & 930 & 10693 & 2.3 & 2.20881 & 4.2 \\
\hline 36 & DM305-42 & 468 & 17941 & 2.0 & 2.63466 & 2.7 \\
\hline 36 & DM305-26 & 178 & 16257 & 2.8 & 2.81156 & 2.3 \\
\hline 36 & DM305-38 & 348 & 13846 & 3.9 & 2.78349 & 3.5 \\
\hline 36 & DM305-8 & 56 & 7915 & 1.9 & 2.89909 & 4.5 \\
\hline 36 & DM305-14 & 85 & 5519 & 1.3 & 2.77799 & 5.0 \\
\hline 36 & DM305-4 & 475 & 42112 & 3.3 & 2.85618 & 5.8 \\
\hline 36 & DM305-20 & 697 & 84392 & 2.9 & 3.05767 & 3.4 \\
\hline 36 & DM305-25 & 267 & 19190 & 2.8 & 2.86434 & 1.8 \\
\hline 36 & DM305-57 & 80 & 7887 & 1.5 & 2.88469 & 3.5 \\
\hline 36 & DM305-7 & 285 & 27651 & 2.7 & 2.81006 & 1.7 \\
\hline 36 & DM305-47 & 1765 & 82989 & 2.1 & 2.70618 & 3.3 \\
\hline 36 & DM305-10 & 304 & 38875 & 2.6 & 3.05013 & 2.7 \\
\hline 36 & DM305-46 & 204 & 27534 & 3.3 & 2.89923 & 2.3 \\
\hline 36 & DM305-22 & 97 & 8631 & 1.6 & 2.93236 & 3.1 \\
\hline 36 & DM305-58 & 47 & 4672 & 1.2 & 2.79652 & 2.2 \\
\hline 36 & DM305-6 & 129 & 17016 & 2.6 & 2.83445 & 1.9 \\
\hline 36 & DM305-50 & 107 & 14056 & 1.7 & 2.99005 & 3.4 \\
\hline 36 & DM305-9 & 76 & 4884 & 1.4 & 2.84663 & 5.1 \\
\hline 36 & DM305-3 & 129 & 7560 & 1.4 & 2.89312 & 7.3 \\
\hline 36 & DM305-12 & 1202 & 3236 & 1.4 & 1.19801 & 7.7 \\
\hline 36 & DM305-24 & 504 & 64988 & 3.0 & 4.13541 & 1.6 \\
\hline 36 & DM305-1 & 239 & 12404 & 2.4 & 4.02357 & 5.5 \\
\hline 36 & DM305-56 & 169 & 22543 & 1.7 & 4.31850 & 1.6 \\
\hline 36 & DM305-36 & 215 & 3888 & 2.6 & 3.85567 & 2.6 \\
\hline 36 & DM305-49 & 727 & 15152 & 4.1 & 3.70660 & 5.8 \\
\hline 36 & DM305-35 & 811 & 3785 & 1.8 & 4.34747 & 3.7 \\
\hline 36 & DM305-18 & 52 & 11561 & 1.5 & 4.91051 & 2.2 \\
\hline 36 & DM305-30 & 270 & 19335 & 2.7 & 7.26820 & 13.3 \\
\hline 36 & DM305-55 & 92 & 11462 & 1.9 & 11.31064 & 2.2 \\
\hline 36 & DM305-11 & 136 & 31652 & 3.0 & 16.41856 & 2.8 \\
\hline 37 & FC063-35 & 1096 & 4857 & 6.0 & 0.10941 & 7.9 \\
\hline 37 & FC063-7 & 1587 & 6796 & 2.4 & 0.09990 & 6.0 \\
\hline 37 & FC063-56 & 1850 & 7765 & 2.2 & 0.10320 & 4.0 \\
\hline 37 & FC063-22 & 1679 & 7498 & 4.2 & 0.10513 & 5.3 \\
\hline 37 & FC063-32 & 1334 & 6918 & 2.4 & 0.10330 & 7.3 \\
\hline 37 & FC063-39 & 1470 & 4893 & 3.0 & 0.10908 & 4.6 \\
\hline 37 & FC063-31 & 1564 & 6994 & 1.8 & 0.10076 & 4.1 \\
\hline 37 & FC063-50 & 1074 & 5364 & 21.3 & 0.10990 & 4.6 \\
\hline 37 & FC063-45 & 924 & 4397 & 1.8 & 0.10720 & 4.3 \\
\hline 37 & FC063-3 & 1752 & 7900 & 3.2 & 0.08769 & 3.5 \\
\hline 37 & FC063-9 & 691 & 4231 & 4.9 & 0.11093 & 9.5 \\
\hline 37 & FC063-48 & 903 & 5870 & 2.3 & 0.11427 & 5.2 \\
\hline 37 & FC063-47 & 1538 & 7089 & 2.1 & 0.10740 & 5.7 \\
\hline 37 & FC063-20 & 732 & 3204 & 2.6 & 0.11098 & 4.2 \\
\hline 37 & FC063-33 & 1984 & 8600 & 4.2 & 0.10724 & 2.4 \\
\hline 37 & FC063-19 & 1422 & 7711 & 2.3 & 0.11013 & 2.1 \\
\hline 37 & FC063-8 & 892 & 5838 & 2.4 & 0.11395 & 3.1 \\
\hline 37 & FC063-18 & 624 & 2958 & 2.4 & 0.11635 & 4.1 \\
\hline 37 & FC063-21 & 633 & 4638 & 2.8 & 0.12171 & 6.6 \\
\hline 37 & FC063-25 & 3122 & 19970 & 2.7 & 0.10862 & 2.1 \\
\hline 37 & FC063-42 & 724 & 3207 & 7.8 & 0.13742 & 5.0 \\
\hline
\end{tabular}


Kimbrough, D.L., Grove, M., Gehrels, G.E., Dorsey, R.J., Howard, K.A., Lovera, O., Aslan, A., House, P.K., and Pearthree, P.A., 2015, Detrital zircon U-Pb provenance of the Colorado River: A 5 m.y. record of incision into cover strata overlying the Colorado Plateau and adjacent regions: Geosphere, v. 11, doi:10.1130/GES00982.1.

TABLE DR7. U-Pb ZIRCON LA-ICP-MS GEOCHRONOLOGIC ANALYSES OF COLORADO RIVER BASIN \& ASSOCIATED SAMPLES.

\begin{tabular}{|c|c|c|c|c|c|c|}
\hline & & & & & & topic ra \\
\hline ID\# & Analysis & $\begin{array}{c}U \\
(\mathrm{ppm})\end{array}$ & $\begin{array}{l}206 \mathrm{~Pb} \\
204 \mathrm{~Pb}\end{array}$ & $\mathrm{U} / \mathrm{Th}$ & $\begin{array}{c}207 \mathrm{~Pb}^{*} \\
235 \mathrm{U}\end{array}$ & $\begin{array}{c} \pm \\
(\%) \\
\end{array}$ \\
\hline & FC063-37 & 789 & 3754 & 2.1 & 0.18053 & 9.1 \\
\hline 37 & FC063-5 & 448 & 4286 & 2.2 & 0.18487 & 4.3 \\
\hline 37 & FC063-4 & 501 & 5434 & 2.4 & 0.18292 & 4.2 \\
\hline 37 & FC063-49 & 534 & 5535 & 2.2 & 0.18313 & 5.7 \\
\hline 37 & FC063-58 & 553 & 3408 & 2.0 & 0.19042 & 4.0 \\
\hline 37 & FC063-38 & 412 & 4912 & 2.8 & 0.19871 & 3.9 \\
\hline 37 & FC063-54 & 382 & 3605 & 2.0 & 0.19155 & 5.5 \\
\hline 37 & FC063-53 & 333 & 3072 & 2.7 & 0.18719 & 7.3 \\
\hline 37 & FC063-12 & 396 & 4163 & 2.1 & 0.19815 & 3.4 \\
\hline 37 & FC063-55 & 692 & 6786 & 2.2 & 0.18606 & 3.7 \\
\hline 37 & FC063-44 & 516 & 4649 & 2.5 & 0.18997 & 5.4 \\
\hline 37 & FC063-6 & 559 & 4201 & 3.2 & 0.18844 & 3.7 \\
\hline 37 & FC063-23 & 275 & 3095 & 3.0 & 0.20376 & 5.0 \\
\hline 37 & FC063-11 & 532 & 4334 & 2.7 & 0.19241 & 4.7 \\
\hline 37 & FC063-26 & 334 & 3164 & 2.4 & 0.19438 & 5.3 \\
\hline 37 & FC063-43 & 409 & 3808 & 2.2 & 0.19006 & 6.2 \\
\hline 37 & FC063-14 & 248 & 2667 & 3.1 & 0.18845 & 5.4 \\
\hline 37 & FC063-24 & 405 & 4387 & 2.2 & 0.19866 & 5.1 \\
\hline 37 & FC063-15 & 284 & 2584 & 3.8 & 0.19083 & 6.9 \\
\hline 37 & FC063-2 & 246 & 2058 & 3.1 & 0.18320 & 6.2 \\
\hline 37 & FC063-30 & 553 & 4939 & 2.5 & 0.19724 & 5.5 \\
\hline 37 & FC063-51 & 365 & 3288 & 2.2 & 0.19615 & 2.1 \\
\hline 37 & FC063-36 & 319 & 3267 & 4.3 & 0.20243 & 8.3 \\
\hline 37 & FC063-1 & 394 & 3712 & 2.8 & 0.18801 & 7.6 \\
\hline 37 & FC063-46 & 604 & 4767 & 2.3 & 0.20225 & 4.4 \\
\hline 37 & FC063-34 & 552 & 4919 & 2.8 & 0.19680 & 4.2 \\
\hline 37 & FC063-17 & 537 & 2092 & 2.4 & 0.23430 & 32.8 \\
\hline 37 & FC063-10 & 976 & 10572 & 3.7 & 0.19523 & 4.2 \\
\hline 37 & FC063-57 & 810 & 7146 & 1.9 & 0.19420 & 9.1 \\
\hline 37 & FC063-52 & 469 & 6108 & 2.4 & 0.19682 & 7.8 \\
\hline 37 & FC063-27 & 701 & 5283 & 2.0 & 0.20608 & 6.7 \\
\hline 37 & FC063-59 & 1104 & 10120 & 8.5 & 0.20543 & 6.6 \\
\hline 37 & FC063-40 & 728 & 12254 & 2.2 & 0.25637 & 3.1 \\
\hline 37 & FC063-13 & 235 & 11424 & 1.3 & 2.01061 & 2.7 \\
\hline 37 & FC063-41 & 930 & 62025 & 1.1 & 3.24005 & 7.2 \\
\hline 37 & FC063-29 & 525 & 38132 & 3.4 & 3.72102 & 3.7 \\
\hline 37 & FC063-60 & 1234 & 77403 & 1.2 & 3.77714 & 3.1 \\
\hline 38 & FC0501-39 & 596 & 681 & 1.3 & 0.03715 & 16.5 \\
\hline 38 & FC0501-29 & 343 & 835 & 2.1 & 0.03792 & 53.4 \\
\hline 38 & FC0501-59 & 1103 & 617 & 0.5 & 0.05639 & 10.2 \\
\hline 38 & FC0501-42 & 296 & 598 & 0.7 & 0.05604 & 15.9 \\
\hline 38 & FC05-1-27 & 141 & 152 & 1.4 & 0.03004 & 32.9 \\
\hline 38 & FC05-1-3 & 477 & 1446 & 1.8 & 0.03530 & 13.2 \\
\hline 38 & FC0501-17 & 355 & 1013 & 1.1 & 0.04640 & 38.8 \\
\hline 38 & FC0501-44 & 136 & 473 & 0.7 & 0.08757 & 48.4 \\
\hline 38 & FC05-1-25 & 708 & 2078 & 2.9 & 0.04836 & 6.6 \\
\hline 38 & FC0501-2 & 589 & 2318 & 0.6 & 0.10215 & 13.5 \\
\hline 38 & FC05-1-1 & 118 & 1075 & 0.9 & 0.11254 & 11.3 \\
\hline 38 & FC0501-30 & 198 & 1050 & 0.6 & 0.19076 & 11.6 \\
\hline 38 & FC0501-43 & 497 & 6463 & 1.2 & 0.17483 & 4.5 \\
\hline 38 & FC0501-51 & 668 & 8405 & 1.1 & 0.18263 & 6.6 \\
\hline 38 & FC0501-53 & 201 & 3031 & 0.5 & 0.16148 & 21.9 \\
\hline 38 & FC0501-50 & 431 & 2808 & 1.7 & 0.26959 & 10.8 \\
\hline 38 & FC05-1-6 & 436 & 3899 & 1.6 & 0.24537 & 7.1 \\
\hline 38 & FC0501-9 & 345 & 6250 & 1.3 & 0.27399 & 8.3 \\
\hline 38 & FC0501-24 & 381 & 2469 & 3.0 & 0.40597 & 9.7 \\
\hline 38 & FC0501-8 & 884 & 9816 & 1.0 & 0.46054 & 6.9 \\
\hline 38 & FC0501-45 & 227 & 3396 & 1.9 & 0.46619 & 6.7 \\
\hline 38 & FC05-1-22 & 223 & 5588 & 1.8 & 0.48183 & 4.6 \\
\hline 38 & FC05-1-5 & 255 & 7976 & 4.2 & 0.52482 & 3.1 \\
\hline 38 & FC0501-55 & 151 & 5182 & 1.1 & 0.51157 & 8.1 \\
\hline 38 & FC0501-23 & 103 & 2961 & 0.4 & 0.56081 & 8.9 \\
\hline 38 & FC0501-22 & 216 & 5955 & 2.7 & 0.65837 & 3.6 \\
\hline 38 & FC0501-31 & 93 & 1903 & 1.0 & 0.75834 & 10.9 \\
\hline 38 & FC0501-48 & 234 & 12424 & 1.6 & 0.85256 & 4.7 \\
\hline 38 & FC0501-5 & 111 & 9827 & 2.0 & 1.84997 & 3.4 \\
\hline 38 & FC0501-41 & 14 & 1338 & 1.3 & 1.31392 & 29.3 \\
\hline 38 & FC05-1-16 & 145 & 32254 & 3.9 & 1.91957 & 2.4 \\
\hline 38 & FC05-1-9 & 100 & 17350 & 2.3 & 2.06158 & 4.2 \\
\hline 38 & FC05-1-26 & 50 & 4107 & 2.3 & 2.17149 & 5.3 \\
\hline 38 & FC0501-12 & 495 & 9987 & 2.0 & 1.60025 & 8.4 \\
\hline 38 & FC05-1-8 & 658 & 4733 & 1.4 & 1.49797 & 6.7 \\
\hline 38 & FC0501-58 & 82 & 9526 & 2.0 & 2.45354 & 4.4 \\
\hline 38 & FC0501-38 & 334 & 16710 & 2.1 & 2.22023 & 1.8 \\
\hline 38 & FC0501-46 & 52 & 6790 & 0.9 & 3.01104 & 6.7 \\
\hline 38 & FC05-1-10 & 847 & 6618 & 2.2 & 2.62902 & 8.1 \\
\hline 38 & FC05-1-21 & 205 & 36539 & 3.0 & 2.90049 & 2.4 \\
\hline 38 & FC0501-7 & 128 & 11063 & 1.4 & 2.73876 & 4.5 \\
\hline 38 & FC0501-40 & 382 & 22662 & 2.9 & 2.92074 & 3.1 \\
\hline 38 & FC0501-32 & 21 & 582 & 1.4 & 1.84668 & 20.7 \\
\hline 38 & FC0501-28 & 416 & 38825 & 2.5 & 3.13780 & 2.3 \\
\hline 38 & FC05-1-19 & 114 & 33803 & 2.0 & 3.30756 & 4.8 \\
\hline 38 & FC0501-20 & 2771 & 8601 & 3.4 & 2.16167 & 2.5 \\
\hline 38 & FC0501-36 & 613 & 47238 & 1.8 & 3.03509 & 1.3 \\
\hline 38 & FC0501-13 & 427 & 9614 & 2.0 & 2.47624 & 2.3 \\
\hline 38 & FC0501-47 & 433 & 23289 & 2.2 & 2.92249 & 1.7 \\
\hline 38 & FC0501-57 & 718 & 29691 & 1.9 & 2.78954 & 1.7 \\
\hline 38 & FC0501-27 & 401 & 5196 & 1.4 & 2.89210 & 3.6 \\
\hline 38 & FC0501-54 & 71 & 10524 & 0.8 & 3.39003 & 3.4 \\
\hline 38 & FC0501-15 & 516 & 8125 & 2.2 & 2.94078 & 5.7 \\
\hline
\end{tabular}


Kimbrough, D.L., Grove, M., Gehrels, G.E., Dorsey, R.J., Howard, K.A., Lovera, O., Aslan, A., House, P.K., and Pearthree, P.A., 2015, Detrital zircon U-Pb provenance of the Colorado River: A 5 m.y. record of incision into cover strata overlying the Colorado Plateau and adjacent regions: Geosphere, v. 11, doi:10.1130/GES00982.1.

TABLE DR7. U-Pb ZIRCON LA-ICP-MS GEOCHRONOLOGIC ANALYSES OF COLORADO RIVER BASIN \& ASSOCIATED SAMPLES.

\begin{tabular}{|c|c|c|c|c|c|c|}
\hline & & & & & & opic ra \\
\hline ID\# & Analysis & $\begin{array}{c}\text { U } \\
(\mathrm{ppm})\end{array}$ & $\begin{array}{l}206 \mathrm{~Pb} \\
204 \mathrm{~Pb}\end{array}$ & $\mathrm{U} / \mathrm{Th}$ & $\begin{array}{c}207 \mathrm{~Pb}^{*} \\
235 \mathrm{U}\end{array}$ & $\begin{array}{c} \pm \\
(\%) \\
\end{array}$ \\
\hline & FC0501-4 & 347 & 6142 & 2.1 & 2.94770 & 2.5 \\
\hline 38 & FC05-1-30 & 175 & 23619 & 3.3 & 3.61342 & 4.3 \\
\hline 38 & FC0501-16 & 99 & 2235 & 0.8 & 3.13507 & 5.5 \\
\hline 38 & FC05-1-28 & 73 & 16004 & 2.4 & 3.84623 & 6.9 \\
\hline 38 & FC05-1-2 & 95 & 8388 & 1.3 & 4.05431 & 8.3 \\
\hline 38 & FC05-1-17 & 441 & 30515 & 2.8 & 4.11487 & 4.5 \\
\hline 38 & FC05-1-14 & 259 & 51151 & 4.9 & 4.12253 & 2.8 \\
\hline 38 & FC0501-60 & 179 & 2342 & 0.6 & 3.40179 & 3.8 \\
\hline 38 & FC0501-21 & 103 & 6307 & 0.9 & 4.20867 & 3.0 \\
\hline 38 & FC0501-14 & 505 & 48325 & 2.6 & 4.27554 & 1.9 \\
\hline 38 & FC0501-52 & 152 & 19678 & 1.7 & 4.26837 & 3.2 \\
\hline 38 & FC0501-56 & 193 & 24331 & 2.0 & 4.29600 & 3.0 \\
\hline 38 & FC0501-1 & 212 & 11432 & 1.4 & 3.86834 & 2.9 \\
\hline 38 & FC0501-10 & 314 & 21696 & 1.5 & 3.94163 & 2.4 \\
\hline 38 & FC0501-3 & 219 & 17749 & 0.9 & 4.19610 & 2.1 \\
\hline 38 & FC05-1-7 & 330 & 52263 & 1.4 & 4.62077 & 2.4 \\
\hline 38 & FC0501-6 & 317 & 36828 & 1.7 & 4.36049 & 1.1 \\
\hline 38 & FC0501-11 & 191 & 17835 & 1.4 & 4.41808 & 2.2 \\
\hline 38 & FC0501-34 & 318 & 10491 & 1.3 & 3.83476 & 2.4 \\
\hline 38 & FC0501-33 & 278 & 8385 & 1.9 & 3.85581 & 3.6 \\
\hline 38 & FC05-1-23 & 199 & 23494 & 4.9 & 4.89050 & 3.2 \\
\hline 38 & FC0501-37 & 447 & 3530 & 1.3 & 3.15307 & 4.0 \\
\hline 38 & FC05-1-20 & 940 & 138322 & 9.8 & 5.01400 & 3.5 \\
\hline 38 & FC0501-49 & 538 & 35488 & 2.0 & 5.05520 & 2.5 \\
\hline 38 & FC05-1-15 & 193 & 14541 & 1.7 & 5.57997 & 3.5 \\
\hline 38 & FC05-1-12 & 49 & 12689 & 3.9 & 5.74166 & 4.5 \\
\hline 38 & FC0501-35 & 241 & 15613 & 1.0 & 5.41701 & 3.7 \\
\hline 38 & FC05-1-24 & 78 & 12090 & 3.4 & 6.60772 & 3.0 \\
\hline 38 & FC0501-25 & 534 & 6310 & 2.3 & 5.02008 & 4.8 \\
\hline 38 & FC0501-18 & 223 & 12159 & 1.1 & 6.39511 & 2.7 \\
\hline 38 & FC05-1-13 & 16 & 4157 & 0.9 & 8.13364 & 5.5 \\
\hline 38 & FC05-1-4 & 324 & 18927 & 2.1 & 8.25554 & 3.5 \\
\hline 38 & FC05-1-11 & 61 & 24193 & 3.1 & 18.94080 & 3.3 \\
\hline 39 & FC126-49 & 411 & 7569 & 1.4 & 0.0243 & 22.1 \\
\hline 39 & FC126-69 & 367 & 12130 & 2.4 & 0.0311 & 24.2 \\
\hline 39 & FC126-24 & 163 & 2988 & 1.5 & 0.0347 & 22.8 \\
\hline 39 & FC126-3 & 995 & 18910 & 1.4 & 0.0348 & 7.3 \\
\hline 39 & FC126-70 & 490 & 6529 & 1.6 & 0.0488 & 7.0 \\
\hline 39 & FC126-45 & 386 & 15398 & 4.9 & 0.0551 & 10.8 \\
\hline 39 & FC126-55 & 335 & 7369 & 1.1 & 0.0583 & 9.7 \\
\hline 39 & FC126-37 & 45 & 1779 & 0.7 & 0.0799 & 58.0 \\
\hline 39 & FC126-54 & 585 & 55987 & 1.0 & 0.2471 & 2.4 \\
\hline 39 & FC126-2 & 81 & 11722 & 0.6 & 0.2457 & 10.5 \\
\hline 39 & FC126-44 & 101 & 27975 & 0.8 & 0.2831 & 9.3 \\
\hline 39 & FC126-14 & 207 & 21095 & 1.8 & 0.2774 & 4.4 \\
\hline 39 & FC126-28 & 54 & 6433 & 2.9 & 0.2872 & 11.6 \\
\hline 39 & FC126-38 & 152 & 10880 & 1.5 & 0.3185 & 9.2 \\
\hline 39 & FC126-75 & 709 & 7509 & 2.5 & 0.4705 & 1.5 \\
\hline 39 & FC126-31 & 167 & 12740 & 1.1 & 0.5001 & 3.1 \\
\hline 39 & FC126-21 & 200 & 4134 & 0.6 & 0.5073 & 2.9 \\
\hline 39 & FC126-61 & 133 & 61629 & 1.8 & 0.7071 & 3.1 \\
\hline 39 & FC126-73 & 170 & 54125 & 2.2 & 0.8168 & 2.9 \\
\hline 39 & FC126-4 & 91 & 43143 & 1.2 & 0.8092 & 4.1 \\
\hline 39 & FC126-57 & 238 & 65805 & 0.4 & 0.8376 & 1.2 \\
\hline 39 & FC126-62 & 186 & 41428 & 1.5 & 0.8561 & 2.6 \\
\hline 39 & FC126-34 & 416 & 164178 & 2.9 & 1.4800 & 1.7 \\
\hline 39 & FC126-30 & 46 & 10612 & 3.1 & 1.7776 & 5.3 \\
\hline 39 & FC126-47 & 109 & 58556 & 2.7 & 1.8363 & 1.2 \\
\hline 39 & FC126-40 & 215 & 212856 & 2.9 & 1.8506 & 2.4 \\
\hline 39 & FC126-64 & 143 & 84822 & 1.7 & 1.8514 & 2.9 \\
\hline 39 & FC126-26 & 247 & 914457 & 2.3 & 1.9387 & 2.8 \\
\hline 39 & FC126-68 & 313 & 114329 & 3.0 & 1.9650 & 1.0 \\
\hline 39 & FC126-56 & 229 & 201473 & 1.8 & 2.2632 & 2.3 \\
\hline 39 & FC126-10 & 115 & 89555 & 0.3 & 2.3370 & 1.3 \\
\hline 39 & FC126-66 & 209 & 204462 & 1.7 & 2.6667 & 0.9 \\
\hline 39 & FC126-29 & 32 & 23180 & 2.5 & 2.7351 & 4.3 \\
\hline 39 & FC126-33 & 161 & 159079 & 2.0 & 2.9987 & 3.6 \\
\hline 39 & FC126-74 & 736 & 83733 & 5.0 & 3.0249 & 1.1 \\
\hline 39 & FC126-59 & 243 & 32676 & 0.6 & 2.6980 & 4.5 \\
\hline 39 & FC126-39 & 354 & 141051 & 2.7 & 2.9269 & 2.6 \\
\hline 39 & FC126-35 & 83 & 83706 & 0.7 & 3.0689 & 2.7 \\
\hline 39 & FC126-46 & 109 & 47913 & 1.2 & 3.1006 & 1.5 \\
\hline 39 & FC126-19 & 222 & 256196 & 2.3 & 3.1796 & 2.9 \\
\hline 39 & FC126-12 & 111 & 130326 & 0.7 & 3.2269 & 1.5 \\
\hline 39 & FC126-22 & 587 & 102936 & 4.4 & 2.9520 & 2.6 \\
\hline 39 & FC126-15 & 142 & 133001 & 2.2 & 3.3589 & 2.3 \\
\hline 39 & FC126-27 & 108 & 103617 & 1.0 & 3.2887 & 3.1 \\
\hline 39 & FC126-23 & 112 & 62320 & 1.1 & 3.9190 & 7.2 \\
\hline 39 & FC126-36 & 148 & 76940 & 1.3 & 4.0288 & 0.9 \\
\hline 39 & FC126-25 & 240 & 366862 & 1.5 & 4.2030 & 0.8 \\
\hline 39 & FC126-8 & 23 & 50355 & 1.7 & 4.1210 & 2.3 \\
\hline 39 & FC126-43 & 216 & 280231 & 2.8 & 4.2347 & 3.3 \\
\hline 39 & FC126-51 & 62 & 54651 & 0.8 & 4.1618 & 2.1 \\
\hline 39 & FC126-53 & 246 & 284694 & 1.9 & 4.1535 & 3.0 \\
\hline 39 & FC126-11 & 159 & 195911 & 2.5 & 4.2419 & 0.9 \\
\hline 39 & FC126-1 & 328 & 336824 & 2.9 & 4.2135 & 1.4 \\
\hline 39 & FC126-63 & 487 & 23566 & 1.3 & 3.5293 & 4.1 \\
\hline 39 & FC126-48 & 216 & 74621 & 0.9 & 4.3206 & 1.0 \\
\hline 39 & FC126-42 & 105 & 130441 & 0.9 & 4.3237 & 3.2 \\
\hline 39 & FC126-58 & 261 & 46779 & 2.5 & 4.5039 & 4.2 \\
\hline
\end{tabular}


Kimbrough, D.L., Grove, M., Gehrels, G.E., Dorsey, R.J., Howard, K.A., Lovera, O., Aslan, A., House, P.K., and Pearthree, P.A., 2015, Detrital zircon U-Pb provenance of the Colorado River: A 5 m.y. record of incision into cover strata overlying the Colorado Plateau and adjacent regions: Geosphere, v. 11, doi:10.1130/GES00982.1.

TABLE DR7. U-Pb ZIRCON LA-ICP-MS GEOCHRONOLOGIC ANALYSES OF COLORADO RIVER BASIN \& ASSOCIATED SAMPLES.

\begin{tabular}{|c|c|c|c|c|c|c|}
\hline & & & & & & opic ra \\
\hline ID\# & Analysis & $\begin{array}{c}\text { U } \\
(\mathrm{ppm})\end{array}$ & $\begin{array}{l}206 \mathrm{~Pb} \\
204 \mathrm{~Pb}\end{array}$ & U/Th & $\begin{array}{c}207 \mathrm{~Pb}^{*} \\
235 \mathrm{U}\end{array}$ & $\begin{array}{c} \pm \\
(\%)\end{array}$ \\
\hline 39 & FC126-72 & 171 & 133684 & 1.7 & 4.3423 & 2.0 \\
\hline 39 & FC126-41 & 55 & 56990 & 2.0 & 4.3267 & 1.1 \\
\hline 39 & FC126-20 & 186 & 43766 & 3.5 & 4.5349 & 4.9 \\
\hline 39 & FC126-5 & 238 & 11290 & 2.0 & 3.8530 & 1.4 \\
\hline 39 & FC126-71 & 81 & 88014 & 2.8 & 5.2891 & 1.3 \\
\hline 39 & FC126-16 & 116 & 139075 & 2.0 & 6.4202 & 1.0 \\
\hline 39 & FC126-32 & 165 & 208928 & 1.9 & 7.1797 & 2.4 \\
\hline 39 & FC126-17 & 125 & 253920 & 1.6 & 15.9728 & 0.8 \\
\hline 39 & FC126-65 & 157 & 136357 & 1.2 & 14.8432 & 2.1 \\
\hline 40 & FC0502-44 & 735 & 1180 & 0.7 & 0.03166 & 36.5 \\
\hline 40 & FC0502-43 & 1290 & 2923 & 1.8 & 0.04240 & 5.8 \\
\hline 40 & FC0502-52 & 401 & 1926 & 1.5 & 0.07381 & 24.6 \\
\hline 40 & FC0502-36 & 579 & 4710 & 2.4 & 0.12605 & 10.5 \\
\hline 40 & FC0502-32 & 527 & 2725 & 2.6 & 0.16437 & 28.7 \\
\hline 40 & FC0502-8 & 237 & 1256 & 0.5 & 0.19646 & 48.2 \\
\hline 40 & FC0502-56 & 45 & 692 & 0.7 & 0.19366 & 50.9 \\
\hline 40 & FC0502-30 & 483 & 5452 & 1.9 & 0.16250 & 9.5 \\
\hline 40 & FC0502-14 & 544 & 1770 & 0.7 & 0.24075 & 15.1 \\
\hline 40 & FC0502-40 & 418 & 3917 & 1.5 & 0.17853 & 10.4 \\
\hline 40 & FC0502-22 & 299 & 3924 & 1.4 & 0.17746 & 8.8 \\
\hline 40 & FC0502-59 & 80 & 1281 & 0.8 & 0.16453 & 21.0 \\
\hline 40 & FC0502-38 & 254 & 4638 & 1.9 & 0.23629 & 13.8 \\
\hline 40 & FC0502-9 & 168 & 3059 & 1.4 & 0.30675 & 8.8 \\
\hline 40 & FC0502-50 & 360 & 11386 & 1.3 & 0.49677 & 5.1 \\
\hline 40 & FC0502-28 & 116 & 5252 & 1.2 & 0.66933 & 7.6 \\
\hline 40 & FC0502-13 & 194 & 8287 & 1.5 & 0.75482 & 5.6 \\
\hline 40 & FC0502-15 & 145 & 7400 & 1.9 & 0.87142 & 5.3 \\
\hline 40 & FC0502-11 & 228 & 14737 & 1.5 & 1.60585 & 4.0 \\
\hline 40 & FC0502-6 & 167 & 14390 & 1.7 & 1.76553 & 3.6 \\
\hline 40 & FC0502-46 & 485 & 55469 & 3.3 & 1.82511 & 1.5 \\
\hline 40 & FC0502-39 & 75 & 6168 & 2.1 & 1.82739 & 4.3 \\
\hline 40 & FC0502-17 & 94 & 8669 & 1.7 & 1.88826 & 3.8 \\
\hline 40 & FC0502-26 & 88 & 5128 & 1.1 & 1.84883 & 7.3 \\
\hline 40 & FC0502-12 & 267 & 24398 & 2.0 & 2.08550 & 1.7 \\
\hline 40 & FC0502-4 & 71 & 4840 & 1.3 & 2.26823 & 6.5 \\
\hline 40 & FC0502-5 & 194 & 5269 & 1.1 & 2.10516 & 2.4 \\
\hline 40 & FC0502-10 & 256 & 6295 & 9.2 & 2.55822 & 3.1 \\
\hline 40 & FC0502-42 & 118 & 15151 & 1.2 & 3.27742 & 3.3 \\
\hline 40 & FC0502-7 & 102 & 12856 & 1.4 & 3.13412 & 2.9 \\
\hline 40 & FC0502-31 & 138 & 16861 & 1.4 & 3.12117 & 3.0 \\
\hline 40 & FC0502-57 & 223 & 24547 & 1.3 & 2.96174 & 1.4 \\
\hline 40 & FC0502-21 & 125 & 15173 & 1.2 & 3.20576 & 3.6 \\
\hline 40 & FC0502-51 & 290 & 37727 & 1.8 & 3.04972 & 3.6 \\
\hline 40 & FC0502-24 & 582 & 53505 & 3.1 & 2.99690 & 2.1 \\
\hline 40 & FC0502-60 & 77 & 7630 & 0.9 & 3.18056 & 4.8 \\
\hline 40 & FC0502-35 & 302 & 33138 & 1.9 & 3.26343 & 1.2 \\
\hline 40 & FC0502-47 & 328 & 15793 & 2.0 & 2.91964 & 4.8 \\
\hline 40 & FC0502-29 & 337 & 35117 & 2.1 & 3.28398 & 1.4 \\
\hline 40 & FC0502-45 & 507 & 10302 & 1.3 & 3.24691 & 1.1 \\
\hline 40 & FC0502-41 & 137 & 4656 & 1.2 & 3.23209 & 6.9 \\
\hline 40 & FC0502-54 & 143 & 4740 & 1.3 & 3.55500 & 4.9 \\
\hline 40 & FC0502-23 & 118 & 16482 & 1.7 & 4.04886 & 2.4 \\
\hline 40 & FC0502-2 & 88 & 12604 & 0.9 & 4.16219 & 2.5 \\
\hline 40 & FC0502-25 & 51 & 8284 & 0.9 & 4.23674 & 3.7 \\
\hline 40 & FC0502-27 & 194 & 25326 & 1.1 & 4.43457 & 2.6 \\
\hline 40 & FC0502-1 & 476 & 30954 & 1.9 & 4.34608 & 2.4 \\
\hline 40 & FC0502-53 & 261 & 34240 & 2.5 & 4.38409 & 2.3 \\
\hline 40 & FC0502-58 & 238 & 38049 & 1.9 & 4.39237 & 1.8 \\
\hline 40 & FC0502-55 & 245 & 38237 & 2.2 & 4.31512 & 1.6 \\
\hline 40 & FC0502-16 & 699 & 102927 & 9.5 & 4.07169 & 1.7 \\
\hline 40 & FC0502-34 & 441 & 60788 & 1.9 & 4.49639 & 1.5 \\
\hline 40 & FC0502-49 & 133 & 28611 & 4.2 & 4.50559 & 2.0 \\
\hline 40 & FC0502-18 & 196 & 11550 & 3.2 & 4.51301 & 2.3 \\
\hline 40 & FC0502-3 & 232 & 30814 & 1.2 & 4.48855 & 2.1 \\
\hline 40 & FC0502-20 & 140 & 7865 & 1.2 & 4.48740 & 4.1 \\
\hline 40 & FC0502-33 & 272 & 39909 & 0.9 & 5.56903 & 1.8 \\
\hline 41 & FC127-33 & 276 & 2931 & 0.8 & 0.0301 & 28.9 \\
\hline 41 & FC127-55 & 577 & 16614 & 2.7 & 0.0309 & 12.4 \\
\hline 41 & FC127-58 & 101 & 2528 & 1.1 & 0.0342 & 56.0 \\
\hline 41 & FC127-44 & 567 & 2872 & 0.4 & 0.0362 & 17.4 \\
\hline 41 & FC127-42 & 760 & 17580 & 0.5 & 0.0479 & 13.0 \\
\hline 41 & FC127-43 & 191 & 1270 & 1.5 & 0.0566 & 30.5 \\
\hline 41 & FC127-64 & 84 & 4686 & 0.8 & 0.0560 & 42.4 \\
\hline 41 & FC127-39 & 75 & 1597 & 0.6 & 0.0810 & 46.2 \\
\hline 41 & FC127-11 & 144 & 7801 & 1.7 & 0.0727 & 18.6 \\
\hline 41 & FC127-28 & 302 & 6809 & 0.9 & 0.0917 & 14.0 \\
\hline 41 & FC127-26 & 240 & 7505 & 1.6 & 0.1023 & 7.1 \\
\hline 41 & FC127-10 & 432 & 58448 & 1.1 & 0.1636 & 2.7 \\
\hline 41 & FC127-68 & 445 & 58321 & 1.1 & 0.1790 & 3.8 \\
\hline 41 & FC127-75 & 406 & 86153 & 1.2 & 0.1837 & 2.7 \\
\hline 41 & FC127-35 & 2121 & 92068 & 2.2 & 0.2153 & 1.1 \\
\hline 41 & FC127-4 & 185 & 29804 & 1.0 & 0.2611 & 5.5 \\
\hline 41 & FC127-22 & 161 & 54317 & 1.1 & 0.2815 & 6.0 \\
\hline 41 & FC127-29 & 271 & 28617 & 1.5 & 0.5120 & 3.1 \\
\hline 41 & FC127-15 & 333 & 60976 & 1.5 & 0.5066 & 2.6 \\
\hline 41 & FC127-37 & 119 & 25468 & 1.3 & 0.5033 & 3.7 \\
\hline 41 & FC127-8 & 299 & 89134 & 1.4 & 0.5190 & 1.8 \\
\hline 41 & FC127-31 & 120 & 34512 & 0.6 & 0.5847 & 3.0 \\
\hline 41 & FC127-25 & 107 & 33455 & 0.6 & 0.7217 & 4.0 \\
\hline 41 & FC127-18 & 433 & 111467 & 15.7 & 0.7468 & 1.5 \\
\hline
\end{tabular}


Kimbrough, D.L., Grove, M., Gehrels, G.E., Dorsey, R.J., Howard, K.A., Lovera, O., Aslan, A., House, P.K., and Pearthree, P.A., 2015, Detrital zircon U-Pb provenance of the Colorado River: A 5 m.y. record of incision into cover strata overlying the Colorado Plateau and adjacent regions: Geosphere, v. 11, doi:10.1130/GES00982.1.

TABLE DR7. U-Pb ZIRCON LA-ICP-MS GEOCHRONOLOGIC ANALYSES OF COLORADO RIVER BASIN \& ASSOCIATED SAMPLES.

\begin{tabular}{|c|c|c|c|c|c|c|}
\hline & & & & & & opic ra \\
\hline ID\# & Analysis & $\begin{array}{c}\text { U } \\
(\mathrm{ppm})\end{array}$ & $\begin{array}{l}206 \mathrm{~Pb} \\
204 \mathrm{~Pb}\end{array}$ & $\mathrm{U} / \mathrm{Th}$ & $\begin{array}{c}207 \mathrm{~Pb}^{*} \\
235 \mathrm{U}\end{array}$ & $\begin{array}{c} \pm \\
(\%)\end{array}$ \\
\hline & FC127-65 & 97 & 43843 & 1.3 & 0.8469 & 4.4 \\
\hline 41 & FC127-62 & 152 & 67098 & 2.4 & 0.8551 & 2.5 \\
\hline 41 & FC127-46 & 29 & 22000 & 2.4 & 1.7379 & 5.1 \\
\hline 41 & FC127-63 & 282 & 99429 & 2.3 & 1.8453 & 0.8 \\
\hline 41 & FC127-45 & 202 & 110142 & 1.9 & 1.8602 & 1.0 \\
\hline 41 & FC127-60 & 111 & 136922 & 2.3 & 1.9311 & 1.2 \\
\hline 41 & FC127-50 & 270 & 330721 & 2.0 & 1.9181 & 1.0 \\
\hline 41 & FC127-3 & 110 & 100862 & 2.8 & 1.9104 & 2.2 \\
\hline 41 & FC127-41 & 257 & 88130 & 1.8 & 2.2515 & 1.9 \\
\hline 41 & FC127-27 & 106 & 64647 & 2.0 & 2.1945 & 4.4 \\
\hline 41 & FC127-34 & 160 & 147186 & 1.9 & 2.6006 & 2.5 \\
\hline 41 & FC127-40 & 105 & 102645 & 1.7 & 2.7528 & 2.5 \\
\hline 41 & FC127-49 & 31 & 22852 & 0.9 & 2.8905 & 2.8 \\
\hline 41 & FC127-48 & 95 & 59719 & 2.4 & 2.8798 & 1.1 \\
\hline 41 & FC127-59 & 54 & 16716 & 1.1 & 2.9360 & 1.9 \\
\hline 41 & FC127-24 & 63 & 76208 & 3.2 & 2.9687 & 2.1 \\
\hline 41 & FC127-47 & 63 & 43704 & 0.6 & 2.9767 & 3.2 \\
\hline 41 & FC127-61 & 60 & 65984 & 1.1 & 2.9267 & 1.8 \\
\hline 41 & FC127-67 & 173 & 21742 & 1.1 & 3.1447 & 1.8 \\
\hline 41 & FC127-71 & 85 & 157998 & 1.1 & 3.1204 & 2.0 \\
\hline 41 & FC127-53 & 48 & 22964 & 1.3 & 3.1328 & 1.7 \\
\hline 41 & FC127-32 & 190 & 114013 & 1.9 & 3.1138 & 1.8 \\
\hline 41 & FC127-2 & 75 & 91353 & 2.0 & 3.0845 & 2.2 \\
\hline 41 & FC127-6 & 34 & 12214 & 1.5 & 2.5578 & 6.7 \\
\hline 41 & FC127-57 & 602 & 432043 & 2.5 & 3.4962 & 0.6 \\
\hline 41 & FC127-20 & 131 & 209653 & 3.2 & 4.0981 & 1.4 \\
\hline 41 & FC127-7 & 529 & 591122 & 3.3 & 4.3116 & 3.4 \\
\hline 41 & FC127-69 & 43 & 50227 & 1.1 & 4.1843 & 1.5 \\
\hline 41 & FC127-72 & 133 & 144473 & 1.9 & 4.2498 & 1.5 \\
\hline 41 & FC127-54 & 263 & 245750 & 3.1 & 4.0467 & 1.6 \\
\hline 41 & FC127-56 & 434 & 392871 & 2.4 & 4.2570 & 0.6 \\
\hline 41 & FC127-1 & 284 & 198746 & 4.0 & 4.2069 & 1.4 \\
\hline 41 & FC127-5 & 117 & 108079 & 1.5 & 4.2587 & 2.3 \\
\hline 41 & FC127-21 & 83 & 105012 & 1.4 & 4.5651 & 1.7 \\
\hline 41 & FC127-12 & 1043 & 140936 & 6.2 & 4.1149 & 1.9 \\
\hline 41 & FC127-52 & 280 & 307972 & 1.9 & 4.5747 & 3.1 \\
\hline 41 & FC127-51 & 60 & 47686 & 2.3 & 4.5853 & 3.0 \\
\hline 41 & FC127-73 & 365 & 176962 & 1.7 & 4.5304 & 1.6 \\
\hline 41 & FC127-16 & 102 & 80196 & 1.4 & 4.9501 & 1.4 \\
\hline 41 & FC127-17 & 109 & 149987 & 1.7 & 5.0023 & 7.0 \\
\hline 41 & FC127-23 & 1291 & 111859 & 1.6 & 4.7271 & 1.5 \\
\hline 41 & FC127-74 & 92 & 92363 & 326.4 & 5.0636 & 1.0 \\
\hline 41 & FC127-70 & 149 & 119699 & 0.6 & 5.1246 & 1.1 \\
\hline 41 & FC127-14 & 104 & 61983 & 0.9 & 6.0406 & 0.8 \\
\hline 42 & FC05-3-50 & 281 & 583 & 0.4 & 0.02415 & 11.8 \\
\hline 42 & FC05-3-23 & 925 & 2417 & 1.9 & 0.02744 & 5.6 \\
\hline 42 & FC05-3-12 & 395 & 503 & 0.6 & 0.07933 & 24.8 \\
\hline 42 & FC05-3-39 & 1396 & 2139 & 1.5 & 0.02872 & 6.5 \\
\hline 42 & FC05-3-64 & 354 & 755 & 0.7 & 0.04129 & 16.9 \\
\hline 42 & FC05-3-61 & 420 & 1033 & 1.2 & 0.02985 & 16.8 \\
\hline 42 & FC05-3-59 & 314 & 1150 & 3.0 & 0.03693 & 22.5 \\
\hline 42 & FC05-3-35 & 96 & 98 & 1.3 & 0.04665 & 38.7 \\
\hline 42 & FC05-3-13 & 169 & 287 & 2.6 & 0.08777 & 21.0 \\
\hline 42 & FC05-3-60 & 206 & 213 & 1.5 & 0.06474 & 30.2 \\
\hline 42 & FC05-3-46 & 776 & 2145 & 2.2 & 0.12001 & 6.5 \\
\hline 42 & FC05-3-19 & 405 & 2002 & 1.0 & 0.10718 & 10.6 \\
\hline 42 & FC05-3-13 & 578 & 4001 & 0.7 & 0.08314 & 7.6 \\
\hline 42 & FC05-3-3 & 703 & 4169 & 0.5 & 0.09972 & 6.5 \\
\hline 42 & FC05-3-48 & 79 & 1024 & 0.8 & 0.17462 & 13.8 \\
\hline 42 & FC05-3-65 & 97 & 1995 & 1.1 & 0.18613 & 7.9 \\
\hline 42 & FC05-3-28 & 261 & 3972 & 0.4 & 0.15505 & 5.7 \\
\hline 42 & FC05-3-15 & 465 & 5814 & 0.5 & 0.17472 & 1.8 \\
\hline 42 & FC05-3-22 & 204 & 3217 & 1.4 & 0.18262 & 6.7 \\
\hline 42 & FC05-3-7 & 531 & 590 & 1.5 & 0.11083 & 44.5 \\
\hline 42 & FC05-3-31 & 201 & 2695 & 0.6 & 0.21753 & 6.2 \\
\hline 42 & FC05-3-47 & 403 & 7140 & 0.3 & 0.23093 & 4.0 \\
\hline 42 & FC05-3-21 & 260 & 6207 & 2.0 & 0.35809 & 3.5 \\
\hline 42 & FC05-3-11 & 224 & 4473 & 2.4 & 0.49570 & 16.8 \\
\hline 42 & FC05-3-14 & 1037 & 25706 & 2.1 & 0.42359 & 2.2 \\
\hline 42 & FC05-3-59 & 128 & 2462 & 0.2 & 0.55033 & 21.6 \\
\hline 42 & FC05-3-23 & 705 & 16558 & 2.2 & 0.49068 & 1.7 \\
\hline 42 & FC05-3-17 & 173 & 4544 & 1.7 & 0.48926 & 6.9 \\
\hline 42 & FC05-3-16 & 110 & 3264 & 0.7 & 0.52196 & 7.1 \\
\hline 42 & FC05-3-68 & 870 & 9819 & 0.8 & 0.54514 & 3.2 \\
\hline 42 & FC05-3-54 & 70 & 1987 & 0.4 & 0.62295 & 6.0 \\
\hline 42 & FC05-3-51 & 306 & 3334 & 8.7 & 0.54011 & 6.1 \\
\hline 42 & FC05-3-26 & 95 & 2496 & 0.4 & 0.70124 & 7.0 \\
\hline 42 & FC05-3-9 & 437 & 19042 & 0.6 & 0.62773 & 3.9 \\
\hline 42 & FC05-3-57 & 89 & 2885 & 0.5 & 0.68695 & 7.2 \\
\hline 42 & FC05-3-51 & 25 & 1199 & 0.2 & 0.67262 & 9.7 \\
\hline 42 & FC05-3-30 & 84 & 3365 & 1.3 & 0.72036 & 4.8 \\
\hline 42 & FC05-3-22 & 250 & 8541 & 0.6 & 0.69066 & 6.3 \\
\hline 42 & FC05-3-32 & 43 & 1040 & 1.2 & 0.81710 & 7.3 \\
\hline 42 & FC05-3-41 & 168 & 8662 & 1.3 & 0.78014 & 4.2 \\
\hline 42 & FC05-3-10 & 306 & 15748 & 0.5 & 0.74702 & 3.8 \\
\hline 42 & FC05-3-27 & 265 & 18925 & 2.2 & 0.82025 & 2.9 \\
\hline 42 & FC05-3-58 & 206 & 7413 & 4.0 & 0.79171 & 1.9 \\
\hline 42 & FC05-3-57 & 204 & 2704 & 1.1 & 0.78928 & 6.2 \\
\hline 42 & FC05-3-50 & 357 & 21724 & 2.0 & 0.81827 & 2.7 \\
\hline 42 & FC05-3-6 & 44 & 822 & 3.3 & 0.84844 & 9.7 \\
\hline
\end{tabular}


Kimbrough, D.L., Grove, M., Gehrels, G.E., Dorsey, R.J., Howard, K.A., Lovera, O., Aslan, A., House, P.K., and Pearthree, P.A., 2015, Detrital zircon U-Pb provenance of the Colorado River: A 5 m.y. record of incision into cover strata overlying the Colorado Plateau and adjacent regions: Geosphere, v. 11, doi:10.1130/GES00982.1.

TABLE DR7. U-Pb ZIRCON LA-ICP-MS GEOCHRONOLOGIC ANALYSES OF COLORADO RIVER BASIN \& ASSOCIATED SAMPLES.

\begin{tabular}{|c|c|c|c|c|c|c|}
\hline & & & & & & opic ra \\
\hline ID\# & Analysis & $\begin{array}{c}\text { U } \\
(\mathrm{ppm})\end{array}$ & $\begin{array}{l}206 \mathrm{~Pb} \\
204 \mathrm{~Pb}\end{array}$ & $\mathrm{U} / \mathrm{Th}$ & $\begin{array}{c}207 \mathrm{~Pb}^{*} \\
235 \mathrm{U}\end{array}$ & $\begin{array}{c} \pm \\
(\%) \\
\end{array}$ \\
\hline 42 & FC05-3-49 & 160 & 7180 & 0.3 & 0.83275 & 1.4 \\
\hline 42 & FC05-3-49 & 169 & 3266 & 1.1 & 0.86946 & 4.1 \\
\hline 42 & FC05-3-52 & 153 & 2571 & 2.6 & 0.89302 & 3.2 \\
\hline 42 & FC05-3-56 & 360 & 15204 & 0.5 & 0.90685 & 4.4 \\
\hline 42 & FC05-3-9 & 157 & 17523 & 3.4 & 1.73637 & 3.0 \\
\hline 42 & FC05-3-36 & 614 & 49833 & 6.9 & 1.74967 & 1.6 \\
\hline 42 & FC05-3-69 & 249 & 22847 & 0.8 & 1.83562 & 1.5 \\
\hline 42 & FC05-3-38 & 135 & 2093 & 3.0 & 1.75844 & 3.5 \\
\hline 42 & FC05-3-58 & 561 & 13555 & 1.7 & 1.50005 & 4.0 \\
\hline 42 & FC05-3-20 & 471 & 46728 & 1.9 & 1.96687 & 3.6 \\
\hline 42 & FC05-3-15 & 175 & 14130 & 1.2 & 2.00187 & 3.6 \\
\hline 42 & FC05-3-35 & 380 & 40991 & 1.2 & 2.14701 & 2.8 \\
\hline 42 & FC05-3-8 & 680 & 14351 & 1.4 & 1.55961 & 4.2 \\
\hline 42 & FC05-3-36 & 360 & 8514 & 2.5 & 2.11651 & 2.3 \\
\hline 42 & FC05-3-1 & 427 & 39816 & 6.0 & 2.01981 & 3.3 \\
\hline 42 & FC05-3-16 & 181 & 26520 & 3.7 & 2.17647 & 1.5 \\
\hline 42 & FC05-3-75 & 142 & 15439 & 0.9 & 2.21991 & 2.3 \\
\hline 42 & FC05-3-64 & 157 & 10867 & 1.6 & 2.27887 & 1.6 \\
\hline 42 & FC05-3-45 & 283 & 16856 & 3.9 & 2.27905 & 2.2 \\
\hline 42 & FC05-3-62 & 192 & 24953 & 7.1 & 2.21239 & 2.8 \\
\hline 42 & FC05-3-12 & 333 & 37348 & 5.0 & 2.41391 & 2.4 \\
\hline 42 & FC05-3-24 & 362 & 4201 & 8.8 & 2.66717 & 3.7 \\
\hline 42 & FC05-3-61 & 239 & 7978 & 1.6 & 2.42736 & 5.1 \\
\hline 42 & FC05-3-7 & 30 & 4559 & 0.3 & 3.02903 & 5.3 \\
\hline 42 & FC05-3-34 & 528 & 32860 & 0.7 & 2.75998 & 2.8 \\
\hline 42 & FC05-3-46 & 500 & 19705 & 2.4 & 2.79261 & 2.1 \\
\hline 42 & FC05-3-63 & 213 & 26863 & 1.4 & 2.99864 & 2.7 \\
\hline 42 & FC05-3-39 & 206 & 16912 & 1.0 & 2.71909 & 3.7 \\
\hline 42 & FC05-3-19 & 296 & 37193 & 4.5 & 3.08609 & 1.6 \\
\hline 42 & FC05-3-33 & 213 & 36562 & 2.6 & 3.00910 & 2.1 \\
\hline 42 & FC05-3-32 & 157 & 15111 & 0.8 & 2.97981 & 3.0 \\
\hline 42 & FC05-3-66 & 377 & 45622 & 0.9 & 3.28172 & 3.0 \\
\hline 42 & FC05-3-74 & 119 & 9718 & 1.2 & 2.83413 & 3.9 \\
\hline 42 & FC05-3-71 & 104 & 12244 & 0.8 & 3.26999 & 2.1 \\
\hline 42 & FC05-3-42 & 117 & 9793 & 1.6 & 3.09361 & 3.7 \\
\hline 42 & FC05-3-2 & 136 & 16848 & 0.8 & 3.36941 & 3.9 \\
\hline 42 & FC05-3-26 & 218 & 37378 & 1.4 & 3.40452 & 2.3 \\
\hline 42 & FC05-3-20 & 447 & 27456 & 3.3 & 3.44835 & 3.1 \\
\hline 42 & FC05-3-30 & 126 & 16144 & 1.2 & 3.49525 & 1.5 \\
\hline 42 & FC05-3-29 & 441 & 15353 & 2.1 & 3.49237 & 2.8 \\
\hline 42 & FC05-3-6 & 191 & 24469 & 1.6 & 3.50171 & 3.8 \\
\hline 42 & FC05-3-5 & 177 & 24789 & 6.1 & 3.54630 & 1.5 \\
\hline 42 & FC05-3-48 & 198 & 25021 & 1.1 & 3.66972 & 1.7 \\
\hline 42 & FC05-3-44 & 199 & 25712 & 1.1 & 3.59851 & 2.5 \\
\hline 42 & FC05-3-65 & 285 & 19674 & 1.5 & 3.45020 & 2.2 \\
\hline 42 & FC05-3-2 & 309 & 44531 & 3.1 & 3.93154 & 1.9 \\
\hline 42 & FC05-3-33 & 166 & 24797 & 1.3 & 4.15922 & 4.7 \\
\hline 42 & FC05-3-52 & 110 & 15230 & 1.0 & 4.21406 & 2.7 \\
\hline 42 & FC05-3-67 & 194 & 24516 & 0.9 & 4.16705 & 2.8 \\
\hline 42 & FC05-3-37 & 170 & 11128 & 1.8 & 4.17859 & 1.6 \\
\hline 42 & FC05-3-63 & 261 & 28546 & 2.2 & 4.11541 & 3.2 \\
\hline 42 & FC05-3-53 & 142 & 10569 & 0.6 & 2.92683 & 5.0 \\
\hline 42 & FC05-3-44 & 335 & 42825 & 4.2 & 4.15235 & 2.2 \\
\hline 42 & FC05-3-41 & 91 & 18628 & 2.5 & 4.32015 & 2.4 \\
\hline 42 & FC05-3-31 & 284 & 3660 & 1.7 & 3.87852 & 5.4 \\
\hline 42 & FC05-3-3 & 593 & 50606 & 3.2 & 4.38511 & 1.7 \\
\hline 42 & FC05-3-5 & 1272 & 34392 & 0.5 & 4.63952 & 4.0 \\
\hline 42 & FC05-3-18 & 258 & 32402 & 0.9 & 4.30917 & 3.8 \\
\hline 42 & FC05-3-18 & 388 & 30878 & 6.3 & 4.51706 & 3.3 \\
\hline 42 & FC05-3-42 & 305 & 28319 & 1.0 & 4.27133 & 2.5 \\
\hline 42 & FC05-3-24 & 508 & 70006 & 1.1 & 4.43077 & 3.8 \\
\hline 42 & FC05-3-62 & 312 & 19139 & 0.7 & 4.11792 & 4.2 \\
\hline 42 & FC05-3-40 & 1005 & 38253 & 2.3 & 4.77073 & 10.2 \\
\hline 42 & FC05-3-4 & 215 & 44515 & 4.1 & 4.64260 & 1.8 \\
\hline 42 & FC05-3-27 & 268 & 36818 & 2.2 & 4.72681 & 4.3 \\
\hline 42 & FC05-3-17 & 136 & 33776 & 2.6 & 4.81485 & 3.8 \\
\hline 42 & FC05-3-34 & 418 & 13498 & 3.1 & 4.28041 & 9.0 \\
\hline 42 & FC05-3-40 & 182 & 19993 & 24.8 & 5.01591 & 2.0 \\
\hline 42 & FC05-3-14 & 326 & 32920 & 0.5 & 6.17680 & 2.1 \\
\hline 42 & FC05-3-54 & 374 & 22912 & 10.4 & 6.30068 & 2.7 \\
\hline 42 & FC05-3-55 & 99 & 16598 & 1.6 & 6.49746 & 2.5 \\
\hline 42 & FC05-3-56 & 155 & 1604 & 3.5 & 6.87039 & 3.5 \\
\hline 42 & FC05-3-28 & 259 & 43304 & 4.5 & 6.42436 & 3.7 \\
\hline 42 & FC05-3-70 & 225 & 37149 & 0.5 & 6.26913 & 5.0 \\
\hline 42 & FC05-3-72 & 632 & 18084 & 0.5 & 5.26805 & 3.9 \\
\hline 42 & FC05-3-43 & 360 & 86673 & 1.1 & 10.98169 & 3.1 \\
\hline 42 & FC05-3-55 & 75 & 24092 & 0.6 & 13.39562 & 2.3 \\
\hline 42 & FC05-3-10 & 53 & 18090 & 2.4 & 13.01485 & 2.1 \\
\hline 43 & FC0601-62 & 1244 & 3151 & 1.1 & 0.02040 & 7.7 \\
\hline 43 & FC06-1-5 & 1518 & 2362 & 0.4 & 0.02546 & 8.7 \\
\hline 43 & FC06-1-52 & 225 & 347 & 0.5 & 0.02999 & 21.5 \\
\hline 43 & FC0601-34 & 320 & 987 & 0.4 & 0.03281 & 23.3 \\
\hline 43 & FC06-1-29 & 303 & 1118 & 1.8 & 0.02860 & 12.9 \\
\hline 43 & FC0601-17 & 1218 & 6220 & 1.0 & 0.04992 & 5.1 \\
\hline 43 & FC06-1-31 & 318 & 222 & 0.9 & 0.07663 & 7.9 \\
\hline 43 & FC06-1-7 & 622 & 2254 & 2.4 & 0.08432 & 11.1 \\
\hline 43 & FC06-1-19 & 414 & 5344 & 1.6 & 0.09581 & 4.4 \\
\hline 43 & FC0601-71 & 119 & 1465 & 0.7 & 0.12170 & 17.2 \\
\hline 43 & FC0601-11 & 353 & 3284 & 1.4 & 0.12700 & 11.7 \\
\hline 43 & FC06-1-120 & 386 & 1889 & 1.2 & 0.18239 & 8.4 \\
\hline
\end{tabular}


Kimbrough, D.L., Grove, M., Gehrels, G.E., Dorsey, R.J., Howard, K.A., Lovera, O., Aslan, A., House, P.K., and Pearthree, P.A., 2015, Detrital zircon U-Pb provenance of the Colorado River: A 5 m.y. record of incision into cover strata overlying the Colorado Plateau and adjacent regions: Geosphere, v. 11, doi:10.1130/GES00982.1.

TABLE DR7. U-Pb ZIRCON LA-ICP-MS GEOCHRONOLOGIC ANALYSES OF COLORADO RIVER BASIN \& ASSOCIATED SAMPLES.

\begin{tabular}{|c|c|c|c|c|c|c|}
\hline & & & & & & opic ra \\
\hline ID\# & $\neq$ Analysis & $\begin{array}{c}U \\
(\mathrm{ppm})\end{array}$ & $\begin{array}{l}206 \mathrm{~Pb} \\
204 \mathrm{~Pb} \\
\end{array}$ & U/Th & $\begin{array}{c}207 \mathrm{~Pb}^{*} \\
235 \mathrm{U}\end{array}$ & $\begin{array}{c} \pm \\
(\%) \\
\end{array}$ \\
\hline & FC0601-54 & 368 & 7058 & 2.2 & 0.16779 & 4.3 \\
\hline 43 & FC0601-38 & 293 & 5799 & 0.6 & 0.17377 & 6.4 \\
\hline 43 & FC0601-24 & 140 & 3248 & 0.8 & 0.17447 & 12.0 \\
\hline 43 & FC0601-31 & 111 & 2513 & 0.4 & 0.19023 & 8.5 \\
\hline 43 & FC0601-6 & 627 & 11374 & 0.8 & 0.18333 & 3.2 \\
\hline 43 & FC06-1-50 & 261 & 445 & 1.5 & 0.20997 & 12.6 \\
\hline 43 & FC06-1-15 & 637 & 2471 & 1.0 & 0.38741 & 2.7 \\
\hline 43 & FC0601-36 & 208 & 10099 & 1.9 & 0.43491 & 4.8 \\
\hline 43 & FC06-1-9 & 411 & 1026 & 2.1 & 0.58294 & 10.3 \\
\hline 43 & FC0601-30 & 421 & 26000 & 2.1 & 0.54632 & 2.9 \\
\hline 43 & FC0601-4 & 230 & 1242 & 1.3 & 0.96754 & 7.6 \\
\hline 43 & FC06-1-33 & 471 & 19810 & 5.2 & 0.66200 & 3.6 \\
\hline 43 & FC0601-66 & 133 & 8520 & 1.7 & 0.67753 & 4.5 \\
\hline 43 & FC0601-22 & 595 & 5425 & 0.9 & 0.82466 & 14.4 \\
\hline 43 & FC0601-52 & 1144 & 20952 & 1.1 & 0.74283 & 6.2 \\
\hline 43 & FC06-1-11 & 385 & 20864 & 2.1 & 0.79884 & 2.5 \\
\hline 43 & FC06-1-2 & 206 & 9066 & 2.0 & 0.76205 & 4.0 \\
\hline 43 & FC0601-53 & 148 & 7384 & 0.9 & 0.86364 & 7.1 \\
\hline 43 & FC06-1-28 & 584 & 25634 & 10.9 & 0.84991 & 2.6 \\
\hline 43 & FC0601-35 & 85 & 6421 & 0.9 & 0.85324 & 4.9 \\
\hline 43 & FC0601-61 & 198 & 19103 & 3.9 & 0.86704 & 3.0 \\
\hline 43 & FC06-1-34 & 97 & 9427 & 1.1 & 1.26446 & 3.1 \\
\hline 43 & FC0601-5 & 84 & 9840 & 2.0 & 1.54872 & 2.2 \\
\hline 43 & FC06-1-53 & 131 & 10448 & 3.1 & 1.50311 & 1.9 \\
\hline 43 & FC06-1-46 & 260 & 18809 & 0.7 & 1.63082 & 1.9 \\
\hline 43 & FC0601-49 & 71 & 9221 & 1.7 & 1.83202 & 4.7 \\
\hline 43 & FC06-1-41 & 278 & 7594 & 3.7 & 1.82163 & 5.0 \\
\hline 43 & FC0601-32 & 135 & 18343 & 1.9 & 1.67760 & 2.2 \\
\hline 43 & FC0601-75 & 228 & 23894 & 2.2 & 1.68943 & 2.8 \\
\hline 43 & FC0601-60 & 199 & 22022 & 3.1 & 1.74568 & 1.6 \\
\hline 43 & FC0601-13 & 228 & 31358 & 5.0 & 1.79407 & 1.8 \\
\hline 43 & FC0601-18 & 329 & 31089 & 1.7 & 1.62158 & 1.8 \\
\hline 43 & FC0601-19 & 69 & 9960 & 1.0 & 1.91939 & 2.3 \\
\hline 43 & FC06-1-54 & 135 & 8474 & 1.7 & 1.63847 & 3.1 \\
\hline 43 & FC0601-28 & 729 & 17485 & 3.3 & 1.59223 & 3.7 \\
\hline 43 & FC06-1-21 & 232 & 35617 & 3.1 & 1.73619 & 1.8 \\
\hline 43 & FC0601-37 & 69 & 10907 & 1.4 & 1.93693 & 3.2 \\
\hline 43 & FC0601-23 & 127 & 9950 & 1.7 & 1.81531 & 2.6 \\
\hline 43 & FC0601-20 & 122 & 13335 & 2.2 & 2.05552 & 4.6 \\
\hline 43 & FC0601-8 & 262 & 12968 & 1.5 & 1.87352 & 2.3 \\
\hline 43 & FC06-1-14 & 269 & 14529 & 10.4 & 1.86904 & 2.0 \\
\hline 43 & FC06-1-17 & 87 & 5828 & 1.1 & 1.95180 & 2.6 \\
\hline 43 & FC0601-43 & 156 & 19669 & 2.2 & 2.21161 & 2.6 \\
\hline 43 & FC0601-2 & 119 & 18163 & 2.2 & 2.12197 & 2.0 \\
\hline 43 & FC0601-73 & 65 & 10379 & 1.0 & 2.32543 & 4.0 \\
\hline 43 & FC06-1-23 & 54 & 7355 & 2.3 & 2.06668 & 2.8 \\
\hline 43 & FC0601-46 & 155 & 25797 & 2.8 & 2.34406 & 2.2 \\
\hline 43 & FC0601-26 & 125 & 12678 & 1.8 & 2.60727 & 2.1 \\
\hline 43 & FC0601-9 & 1021 & 60121 & 2.7 & 2.60395 & 2.4 \\
\hline 43 & FC0601-42 & 130 & 23293 & 1.7 & 2.83820 & 3.8 \\
\hline 43 & FC06-1-24 & 251 & 30374 & 2.1 & 2.39736 & 5.7 \\
\hline 43 & FC0601-65 & 375 & 16552 & 1.6 & 2.64319 & 5.9 \\
\hline 43 & FC0601-10 & 92 & 14342 & 2.0 & 2.67744 & 1.7 \\
\hline 43 & FC0601-58 & 148 & 26847 & 1.4 & 2.99718 & 1.8 \\
\hline 43 & FC0601-57 & 231 & 42464 & 1.1 & 2.86796 & 1.5 \\
\hline 43 & FC0601-69 & 73 & 14791 & 1.1 & 3.04434 & 2.1 \\
\hline 43 & FC06-1-27 & 174 & 23846 & 1.9 & 2.92354 & 2.0 \\
\hline 43 & FC0601-7 & 235 & 41216 & 1.3 & 2.91227 & 1.7 \\
\hline 43 & FC06-1-25 & 223 & 16419 & 1.3 & 2.98329 & 3.6 \\
\hline 43 & FC06-1-30 & 34 & 3991 & 0.5 & 2.91627 & 3.3 \\
\hline 43 & FC0601-40 & 92 & 20976 & 1.6 & 3.24536 & 2.4 \\
\hline 43 & FC06-1-4 & 373 & 43133 & 1.4 & 3.00444 & 2.7 \\
\hline 43 & FC0601-74A & 76 & 15423 & 1.5 & 3.00704 & 2.1 \\
\hline 43 & FC0601-14 & 309 & 42076 & 1.7 & 3.12938 & 2.3 \\
\hline 43 & FC06-1-3 & 160 & 12626 & 1.5 & 2.85946 & 2.8 \\
\hline 43 & FC0601-56 & 16 & 8547 & 83.1 & 3.28524 & 6.9 \\
\hline 43 & FC0601-21 & 79 & 17408 & 1.2 & 3.14837 & 2.0 \\
\hline 43 & FC06-1-22 & 310 & 15983 & 1.3 & 2.95656 & 3.9 \\
\hline 43 & FC06-1-36 & 208 & 10054 & 1.1 & 2.98961 & 3.0 \\
\hline 43 & FC06-1-32 & 471 & 3247 & 2.1 & 3.21204 & 4.3 \\
\hline 43 & FC06-1-8 & 214 & 36708 & 2.1 & 3.16464 & 2.7 \\
\hline 43 & FC06-1-12 & 80 & 8371 & 0.9 & 3.14270 & 10.2 \\
\hline 43 & FC06-1-39 & 939 & 100410 & 27.1 & 3.58277 & 1.8 \\
\hline 43 & FC0601-59 & 143 & 8327 & 1.8 & 3.78226 & 1.9 \\
\hline 43 & FC06-1-43 & 305 & 5759 & 1.4 & 3.06729 & 4.9 \\
\hline 43 & FC06-1-16 & 52 & 8255 & 0.8 & 3.95202 & 2.9 \\
\hline 43 & FC0601-12 & 125 & 30719 & 1.9 & 4.14531 & 1.6 \\
\hline 43 & FC06-1-45 & 137 & 12250 & 1.3 & 3.97440 & 1.5 \\
\hline 43 & FC06-1-47 & 189 & 20732 & 0.4 & 4.06645 & 2.1 \\
\hline 43 & FC06-1-37 & 147 & 17993 & 1.3 & 4.04560 & 3.3 \\
\hline 43 & FC06-1-26 & 411 & 53248 & 1.7 & 4.02613 & 2.5 \\
\hline 43 & FC06-1-10 & 88 & 13929 & 1.3 & 4.18766 & 8.7 \\
\hline 43 & FC0601-67 & 133 & 29004 & 1.7 & 4.33889 & 2.6 \\
\hline 43 & FC0601-3 & 164 & 37682 & 1.4 & 4.37758 & 1.7 \\
\hline 43 & FC0601-48 & 212 & 33044 & 2.2 & 4.02529 & 2.4 \\
\hline 43 & FC0601-55 & 289 & 38320 & 0.9 & 4.19456 & 3.4 \\
\hline 43 & FC0601-44 & 204 & 41494 & 2.3 & 4.49110 & 4.4 \\
\hline 43 & FC0601-33 & 335 & 27938 & 1.8 & 4.48983 & 2.1 \\
\hline 43 & FC06-1-42 & 312 & 18779 & 1.4 & 4.50247 & 2.4 \\
\hline 43 & FC06-1-18 & 436 & 50798 & 3.9 & 4.27747 & 2.2 \\
\hline
\end{tabular}


Kimbrough, D.L., Grove, M., Gehrels, G.E., Dorsey, R.J., Howard, K.A., Lovera, O., Aslan, A., House, P.K., and Pearthree, P.A., 2015, Detrital zircon U-Pb provenance of the Colorado River: A 5 m.y. record of incision into cover strata overlying the Colorado Plateau and adjacent regions: Geosphere, v. 11, doi:10.1130/GES00982.1.

TABLE DR7. U-Pb ZIRCON LA-ICP-MS GEOCHRONOLOGIC ANALYSES OF COLORADO RIVER BASIN \& ASSOCIATED SAMPLES.

\begin{tabular}{|c|c|c|c|c|c|c|}
\hline & & & & & & opic ra \\
\hline ID\# & $\neq$ Analysis & $\begin{array}{c}U \\
(\mathrm{ppm})\end{array}$ & $\begin{array}{l}206 \mathrm{~Pb} \\
204 \mathrm{~Pb} \\
\end{array}$ & U/Th & $\begin{array}{c}207 \mathrm{~Pb}^{*} \\
235 \mathrm{U}\end{array}$ & $\begin{array}{c} \pm \\
(\%) \\
\end{array}$ \\
\hline & FC0601-72 & 466 & 68058 & 1.5 & 4.48006 & 4.1 \\
\hline 43 & FC06-1-44 & 330 & 51370 & 2.2 & 4.66258 & 2.6 \\
\hline 43 & FC0601-16 & 804 & 57562 & 7.4 & 4.06285 & 3.5 \\
\hline 43 & FC0601-15 & 148 & 40419 & 3.5 & 4.84592 & 2.1 \\
\hline 43 & FC0601-70 & 708 & 118601 & 2.0 & 4.65729 & 1.6 \\
\hline 43 & FC0601-39 & 32 & 9457 & 1.2 & 4.76691 & 2.8 \\
\hline 43 & FC06-1-51 & 277 & 34649 & 0.8 & 4.97464 & 1.7 \\
\hline 43 & FC0601-25 & 51 & 16252 & 3.1 & 5.36848 & 2.8 \\
\hline 43 & FC06-1-13 & 465 & 7335 & 1.1 & 3.85654 & 3.7 \\
\hline 43 & FC0601-27 & 205 & 25147 & 2.1 & 5.37465 & 5.6 \\
\hline 43 & FC06-1-48 & 589 & 79166 & 1.6 & 5.84102 & 2.9 \\
\hline 43 & FC0601-1 & 51 & 9674 & 1.2 & 5.67055 & 5.9 \\
\hline 43 & FC0601-29 & 161 & 49353 & 1.9 & 7.23693 & 3.7 \\
\hline 43 & FC0601-45 & 75 & 34960 & 1.3 & 14.35038 & 1.7 \\
\hline 43 & FC06-1-49 & 312 & 77448 & 1.9 & 18.02083 & 2.8 \\
\hline 44 & FC0602-12 & 268 & 839 & 1.3 & 0.03771 & 20.8 \\
\hline 44 & FC0602-43 & 371 & 3544 & 0.6 & 0.06348 & 12.6 \\
\hline 44 & FC06-2-16 & 603 & 2746 & 1.7 & 0.07149 & 7.9 \\
\hline 44 & FC0602-75 & 484 & 4474 & 1.0 & 0.08725 & 6.9 \\
\hline 44 & FC06-2-12 & 278 & 1245 & 1.1 & 0.08858 & 12.0 \\
\hline 44 & FC06-2-52 & 455 & 1030 & 1.2 & 0.09182 & 11.1 \\
\hline 44 & FC0602-69 & 409 & 3500 & 1.8 & 0.10791 & 8.3 \\
\hline 44 & FC0602-28 & 302 & 3436 & 2.6 & 0.10710 & 8.0 \\
\hline 44 & FC06-2-44 & 683 & 7177 & 1.1 & 0.10559 & 5.4 \\
\hline 44 & FC0602-11 & 130 & 2843 & 0.7 & 0.19057 & 9.2 \\
\hline 44 & FC0602-2 & 184 & 6144 & 0.6 & 0.20138 & 6.2 \\
\hline 44 & FC06-2-38 & 282 & 5364 & 1.1 & 0.19773 & 4.8 \\
\hline 44 & FC06-2-8 & 354 & 7138 & 0.7 & 0.20410 & 5.5 \\
\hline 44 & FC06-2-57 & 532 & 13021 & 2.2 & 0.25188 & 5.1 \\
\hline 44 & FC0602-40 & 667 & 9652 & 1.2 & 0.27543 & 2.7 \\
\hline 44 & FC0602-39 & 141 & 4894 & 1.8 & 0.26626 & 6.6 \\
\hline 44 & FC06-2-4 & 486 & 1113 & 1.4 & 0.27410 & 5.7 \\
\hline 44 & FC06-2-47 & 914 & 3467 & 0.6 & 0.29994 & 5.8 \\
\hline 44 & FC0602-24 & 248 & 12047 & 1.1 & 0.40068 & 2.7 \\
\hline 44 & FC06-2-40 & 614 & 2060 & 1.4 & 0.45071 & 6.2 \\
\hline 44 & FC06-2-33 & 159 & 5185 & 0.9 & 0.45997 & 9.9 \\
\hline 44 & FC0602-15 & 218 & 11910 & 2.7 & 0.48606 & 4.0 \\
\hline 44 & FC0602-45 & 330 & 19878 & 3.0 & 0.48695 & 3.6 \\
\hline 44 & FC06-2-35 & 488 & 16239 & 5.3 & 0.51542 & 4.1 \\
\hline 44 & FC06-2-36 & 175 & 4875 & 1.3 & 0.50608 & 3.7 \\
\hline 44 & FC0602-4 & 115 & 8438 & 1.1 & 0.53227 & 5.2 \\
\hline 44 & FC0602-65 & 357 & 10217 & 4.3 & 0.63006 & 5.9 \\
\hline 44 & FC0602-50 & 120 & 8636 & 1.8 & 0.64721 & 5.1 \\
\hline 44 & FC0602-26 & 103 & 8053 & 2.2 & 0.75627 & 3.1 \\
\hline 44 & FC06-2-54 & 291 & 2247 & 0.9 & 0.82908 & 5.5 \\
\hline 44 & FC06-2-30 & 111 & 1360 & 1.8 & 1.00329 & 18.4 \\
\hline 44 & FC06-2-18 & 137 & 7080 & 1.4 & 0.90686 & 3.7 \\
\hline 44 & FC06-2-45 & 94 & 8399 & 1.3 & 1.48449 & 6.1 \\
\hline 44 & FC0602-3 & 34 & 7341 & 2.4 & 1.71196 & 4.5 \\
\hline 44 & FC06-2-48 & 156 & 17652 & 1.6 & 1.67187 & 4.1 \\
\hline 44 & FC06-2-41 & 213 & 26137 & 0.8 & 1.70054 & 2.2 \\
\hline 44 & FC0602-19 & 117 & 18186 & 2.3 & 1.76678 & 2.7 \\
\hline 44 & FC0602-51 & 187 & 27624 & 3.1 & 1.72283 & 1.4 \\
\hline 44 & FC0602-31 & 125 & 21744 & 2.1 & 1.69145 & 5.1 \\
\hline 44 & $\mathrm{FC} 0602-68$ & 134 & 13761 & 2.2 & 1.64760 & 2.7 \\
\hline 44 & FC0602-33 & 190 & 23825 & 2.6 & 1.77512 & 3.6 \\
\hline 44 & FC0602-30 & 103 & 16073 & 1.8 & 1.86877 & 2.7 \\
\hline 44 & FC06-2-24 & 248 & 22802 & 2.3 & 1.91788 & 1.8 \\
\hline 44 & FC06-2-53 & 117 & 15776 & 2.7 & 1.90642 & 3.0 \\
\hline 44 & FC0602-7 & 22 & 4693 & 1.3 & 2.03365 & 5.5 \\
\hline 44 & FC06-2-20 & 177 & 8815 & 0.9 & 1.80428 & 3.3 \\
\hline 44 & FC0602-27 & 67 & 9637 & 1.1 & 1.83453 & 2.1 \\
\hline 44 & FC06-2-9 & 132 & 11590 & 1.7 & 2.07864 & 3.8 \\
\hline 44 & FC0602-74 & 198 & 17650 & 1.4 & 1.97754 & 2.4 \\
\hline 44 & FC0602-25 & 196 & 26680 & 1.6 & 1.96244 & 1.4 \\
\hline 44 & FC0602-44 & 127 & 19375 & 1.6 & 1.96426 & 1.6 \\
\hline 44 & FC0602-38 & 247 & 11518 & 0.8 & 1.91550 & 2.9 \\
\hline 44 & FC0602-60 & 206 & 12384 & 1.7 & 1.86058 & 3.0 \\
\hline 44 & FC0602-21 & 220 & 39298 & 1.6 & 2.08370 & 1.6 \\
\hline 44 & FC06-2-42 & 352 & 38975 & 3.5 & 2.15605 & 2.3 \\
\hline 44 & FC0602-22 & 131 & 9333 & 0.8 & 1.86101 & 4.5 \\
\hline 44 & FC06-2-19 & 124 & 14283 & 0.8 & 2.26127 & 2.7 \\
\hline 44 & FC0602-9 & 116 & 23484 & 1.6 & 2.23361 & 2.8 \\
\hline 44 & FC0602-36 & 65 & 10470 & 2.0 & 2.27366 & 4.0 \\
\hline 44 & FC0602-49 & 68 & 6300 & 1.8 & 2.21291 & 4.2 \\
\hline 44 & FC06-2-10 & 90 & 18236 & 1.3 & 2.91674 & 4.8 \\
\hline 44 & FC06-2-14 & 1045 & 79312 & 3.7 & 2.98365 & 3.2 \\
\hline 44 & FC06-2-32 & 99 & 11829 & 1.9 & 2.93954 & 3.5 \\
\hline 44 & FC06-2-55 & 224 & 27028 & 3.2 & 3.07459 & 3.9 \\
\hline 44 & FC06-2-43 & 362 & 40825 & 1.7 & 3.05282 & 1.7 \\
\hline 44 & FC0602-53 & 192 & 36500 & 2.3 & 2.94782 & 1.9 \\
\hline 44 & FC0602-73 & 409 & 62892 & 2.0 & 3.05837 & 5.8 \\
\hline 44 & FC06-2-21 & 372 & 12639 & 1.6 & 2.92082 & 4.1 \\
\hline 44 & FC06-2-3 & 104 & 8592 & 1.1 & 3.15974 & 4.9 \\
\hline 44 & FC0602-47 & 144 & 16570 & 2.1 & 2.69022 & 3.1 \\
\hline 44 & $\mathrm{FC} 0602-23$ & 30 & 8181 & 1.0 & 3.13049 & 2.8 \\
\hline 44 & FC06-2-49 & 319 & 8734 & 2.9 & 3.24631 & 3.0 \\
\hline 44 & FC06-2-37 & 1744 & 21306 & 2.0 & 3.33166 & 2.4 \\
\hline 44 & FC0602-61 & 90 & 17325 & 2.1 & 3.46854 & 3.9 \\
\hline 44 & FC0602-10 & 128 & 36727 & 3.8 & 3.60591 & 3.6 \\
\hline
\end{tabular}


Kimbrough, D.L., Grove, M., Gehrels, G.E., Dorsey, R.J., Howard, K.A., Lovera, O., Aslan, A., House, P.K., and Pearthree, P.A., 2015, Detrital zircon U-Pb provenance of the Colorado River: A 5 m.y. record of incision into cover strata overlying the Colorado Plateau and adjacent regions: Geosphere, v. 11, doi:10.1130/GES00982.1.

TABLE DR7. U-Pb ZIRCON LA-ICP-MS GEOCHRONOLOGIC ANALYSES OF COLORADO RIVER BASIN \& ASSOCIATED SAMPLES.

\begin{tabular}{|c|c|c|c|c|c|c|}
\hline & & & & & & opic ra \\
\hline ID\# & Analysis & $\begin{array}{c}U \\
(\mathrm{ppm})\end{array}$ & $\begin{array}{l}206 \mathrm{~Pb} \\
204 \mathrm{~Pb}\end{array}$ & $\mathrm{U} / \mathrm{Th}$ & $\begin{array}{c}207 \mathrm{~Pb}^{*} \\
235 \mathrm{U}\end{array}$ & $\begin{array}{c} \pm \\
(\%) \\
\end{array}$ \\
\hline 44 & FC06-2-7 & 114 & 17768 & 1.0 & 3.62285 & 3.7 \\
\hline 44 & FC0602-52 & 115 & 32921 & 0.9 & 3.95454 & 1.5 \\
\hline 44 & FC06-2-17 & 830 & 58293 & 1.8 & 4.19011 & 2.0 \\
\hline 44 & FC0602-46 & 262 & 73313 & 1.7 & 4.33545 & 2.4 \\
\hline 44 & FC0602-66 & 81 & 20305 & 0.8 & 4.17368 & 1.6 \\
\hline 44 & FC0602-6 & 489 & 156396 & 7.2 & 4.16471 & 1.9 \\
\hline 44 & FC0602-67 & 47 & 12730 & 0.9 & 4.24984 & 1.6 \\
\hline 44 & FC0602-58 & 262 & 58730 & 1.4 & 4.14650 & 1.9 \\
\hline 44 & FC0602-72 & 114 & 16285 & 1.4 & 4.41281 & 3.5 \\
\hline 44 & FC06-2-28 & 221 & 25895 & 1.5 & 4.06144 & 3.7 \\
\hline 44 & FC06-2-51 & 873 & 75303 & 2.1 & 4.34632 & 2.0 \\
\hline 44 & FC06-2-60 & 191 & 16098 & 1.5 & 4.36040 & 3.2 \\
\hline 44 & FC06-2-22 & 54 & 9665 & 1.0 & 4.37189 & 2.4 \\
\hline 44 & FC0602-63 & 145 & 13644 & 1.3 & 4.20888 & 2.1 \\
\hline 44 & FC0602-48 & 112 & 27584 & 0.9 & 4.27855 & 1.6 \\
\hline 44 & FC0602-57 & 206 & 40026 & 1.0 & 4.28866 & 1.7 \\
\hline 44 & FC0602-35 & 61 & 14497 & 1.4 & 4.54548 & 2.2 \\
\hline 44 & FC0602-1 & 306 & 92820 & 2.4 & 4.32887 & 2.6 \\
\hline 44 & FC06-2-59 & 113 & 19472 & 1.3 & 4.34525 & 7.6 \\
\hline 44 & FC0602-71 & 137 & 33207 & 0.9 & 4.42500 & 1.8 \\
\hline 44 & FC06-2-56 & 266 & 36407 & 2.8 & 4.31298 & 1.8 \\
\hline 44 & FC0602-59 & 144 & 35366 & 1.2 & 4.42791 & 1.9 \\
\hline 44 & FC06-2-27 & 324 & 67514 & 2.7 & 4.45348 & 2.4 \\
\hline 44 & FC06-2-11 & 501 & 28500 & 1.6 & 4.35656 & 4.0 \\
\hline 44 & FC0602-29 & 351 & 49072 & 2.4 & 4.61711 & 3.2 \\
\hline 44 & FC0602-20 & 162 & 41964 & 1.4 & 4.23292 & 2.4 \\
\hline 44 & FC06-2-26 & 521 & 81244 & 1.6 & 4.48225 & 2.4 \\
\hline 44 & FC06-2-1 & 177 & 25163 & 3.4 & 4.63233 & 4.1 \\
\hline 44 & FC0602-37 & 86 & 17584 & 2.7 & 4.55411 & 2.1 \\
\hline 44 & FC06-2-29 & 304 & 59880 & 1.7 & 4.66605 & 4.2 \\
\hline 44 & FC0602-54 & 380 & 97686 & 1.9 & 4.60622 & 2.1 \\
\hline 44 & FC06-2-15 & 72 & 10382 & 1.0 & 5.08051 & 3.5 \\
\hline 44 & FC06-2-34 & 85 & 8579 & 1.2 & 5.42144 & 3.9 \\
\hline 44 & FC06-2-5 & 163 & 46948 & 1.7 & 5.58848 & 3.2 \\
\hline 44 & FC0602-70 & 125 & 26891 & 0.9 & 5.37322 & 3.7 \\
\hline 44 & FC0602-13 & 108 & 40701 & 1.6 & 6.65237 & 2.3 \\
\hline 44 & FC06-2-23 & 237 & 62571 & 1.8 & 10.31895 & 1.8 \\
\hline 44 & FC06-2-2 & 58 & 4340 & 1.3 & 9.48213 & 4.6 \\
\hline 44 & FC0602-8 & 271 & 30651 & 0.9 & 11.28006 & 4.8 \\
\hline 45 & $2-4-06-1-25$ & 419 & 1318 & 1.8 & 0.03010 & 11.2 \\
\hline 45 & $2-4-06-1-9$ & 473 & 1165 & 0.5 & 0.03555 & 9.5 \\
\hline 45 & $2-4-06-1-62$ & 413 & 1513 & 0.8 & 0.02685 & 11.3 \\
\hline 45 & $2-4-06-1-43$ & 2173 & 2448 & 2.2 & 0.03745 & 7.0 \\
\hline 45 & $2-4-06-1-60$ & 256 & 877 & 0.5 & 0.04179 & 11.2 \\
\hline 45 & $2-4-06-1-37$ & 99 & 372 & 0.7 & 0.04413 & 42.5 \\
\hline 45 & $2-4-06-1-7$ & 278 & 1044 & 1.1 & 0.04182 & 15.4 \\
\hline 45 & $2-4-06-1-12$ & 302 & 1356 & 0.6 & 0.04018 & 8.9 \\
\hline 45 & $2-4-06-1-68$ & 146 & 604 & 1.6 & 0.05417 & 28.3 \\
\hline 45 & $2-4-06-1-2$ & 411 & 753 & 0.5 & 0.05783 & 13.5 \\
\hline 45 & $2-4-06-1-10$ & 288 & 1199 & 1.4 & 0.04495 & 15.1 \\
\hline 45 & $2-4-06-1-42$ & 213 & 847 & 0.1 & 0.04577 & 13.3 \\
\hline 45 & $2-4-06-1-14$ & 353 & 1725 & 4.1 & 0.05630 & 14.0 \\
\hline 45 & $2-4-06-1-39$ & 322 & 3431 & 1.3 & 0.09032 & 7.3 \\
\hline 45 & 2-4-06-1-15 & 482 & 4056 & 1.5 & 0.10315 & 10.0 \\
\hline 45 & $2-4-06-1-41$ & 173 & 1608 & 1.0 & 0.09888 & 11.4 \\
\hline 45 & $2-4-06-1-23$ & 274 & 2950 & 0.8 & 0.10830 & 5.8 \\
\hline 45 & $2-4-06-1-40$ & 1633 & 7879 & 1.9 & 0.11024 & 2.7 \\
\hline 45 & $2-4-06-1-3$ & 244 & 3176 & 2.2 & 0.11553 & 7.0 \\
\hline 45 & 2-4-06-1-11 & 165 & 2406 & 2.0 & 0.12716 & 8.2 \\
\hline 45 & $2-4-06-1-55$ & 627 & 6963 & 0.9 & 0.11855 & 5.0 \\
\hline 45 & $2-4-06-1-12$ & 461 & 6107 & 0.4 & 0.16838 & 3.0 \\
\hline 45 & $2-4-06-1-45$ & 197 & 3727 & 0.6 & 0.16334 & 4.8 \\
\hline 45 & $2-4-06-1-31$ & 480 & 6133 & 0.7 & 0.16194 & 2.4 \\
\hline 45 & $2-4-06-1-65$ & 128 & 2394 & 1.3 & 0.15868 & 11.9 \\
\hline 45 & $2-4-06-1-18$ & 74 & 1608 & 1.2 & 0.17445 & 14.3 \\
\hline 45 & $2-4-06-1-67$ & 506 & 10233 & 2.3 & 0.17626 & 7.2 \\
\hline 45 & $2-4-06-1-57$ & 401 & 7028 & 0.5 & 0.18605 & 3.8 \\
\hline 45 & $2-4-06-1-2$ & 529 & 5681 & 0.6 & 0.18681 & 4.9 \\
\hline 45 & $2-4-06-1-13$ & 232 & 6473 & 2.1 & 0.23726 & 5.1 \\
\hline 45 & $2-4-06-1-64$ & 222 & 4989 & 0.6 & 0.23840 & 4.9 \\
\hline 45 & $2-4-06-1-16$ & 69 & 1923 & 1.1 & 0.26284 & 15.0 \\
\hline 45 & 2-4-06-1-15 & 148 & 2112 & 0.5 & 0.30936 & 14.7 \\
\hline 45 & $2-4-06-1-35$ & 173 & 4698 & 1.1 & 0.27766 & 7.6 \\
\hline 45 & $2-4-06-1-51$ & 142 & 4906 & 0.6 & 0.30403 & 4.3 \\
\hline 45 & $2-4-06-1-25$ & 102 & 3076 & 0.4 & 0.34047 & 5.2 \\
\hline 45 & $2-4-06-1-59$ & 1692 & 3399 & 1.8 & 0.41734 & 3.0 \\
\hline 45 & $2-4-06-1-56$ & 889 & 4571 & 1.2 & 0.44717 & 5.4 \\
\hline 45 & $2-4-06-1-53$ & 366 & 10556 & 1.5 & 0.43402 & 3.5 \\
\hline 45 & $2-4-06-1-52$ & 171 & 9497 & 0.5 & 0.47226 & 4.2 \\
\hline 45 & $2-4-06-1-37$ & 99 & 5008 & 1.1 & 0.48878 & 7.2 \\
\hline 45 & $2-4-06-1-38$ & 95 & 5212 & 1.1 & 0.49350 & 4.5 \\
\hline 45 & $2-4-06-1-47$ & 293 & 9227 & 1.0 & 0.52101 & 3.7 \\
\hline 45 & $2-4-06-1-48$ & 164 & 2307 & 1.3 & 0.59409 & 6.0 \\
\hline 45 & $2-4-06-1-56$ & 340 & 8601 & 2.0 & 0.51872 & 2.3 \\
\hline 45 & $2-4-06-1-42$ & 163 & 2162 & 1.0 & 0.65217 & 14.5 \\
\hline 45 & $2-4-06-1-21$ & 813 & 10540 & 0.8 & 0.53008 & 2.8 \\
\hline 45 & $2-4-06-1-39$ & 315 & 7495 & 0.8 & 0.55695 & 5.1 \\
\hline 45 & $2-4-06-1-32$ & 96 & 1961 & 1.0 & 0.64974 & 11.4 \\
\hline 45 & $2-4-06-1-24$ & 166 & 1990 & 0.5 & 0.72227 & 9.0 \\
\hline 45 & $2-4-06-1-34$ & 109 & 5874 & 2.0 & 0.60714 & 4.2 \\
\hline
\end{tabular}


Kimbrough, D.L., Grove, M., Gehrels, G.E., Dorsey, R.J., Howard, K.A., Lovera, O., Aslan, A., House, P.K., and Pearthree, P.A., 2015, Detrital zircon U-Pb provenance of the Colorado River: A 5 m.y. record of incision into cover strata overlying the Colorado Plateau and adjacent regions: Geosphere, v. 11, doi:10.1130/GES00982.1.

TABLE DR7. U-Pb ZIRCON LA-ICP-MS GEOCHRONOLOGIC ANALYSES OF COLORADO RIVER BASIN \& ASSOCIATED SAMPLES.

\begin{tabular}{|c|c|c|c|c|c|c|}
\hline & & & & & & opic ra \\
\hline ID\# & Analysis & $\begin{array}{c}\text { U } \\
(\mathrm{ppm})\end{array}$ & $\begin{array}{l}206 \mathrm{~Pb} \\
204 \mathrm{~Pb}\end{array}$ & $\mathrm{U} / \mathrm{Th}$ & $\begin{array}{c}207 \mathrm{~Pb}^{*} \\
235 \mathrm{U}\end{array}$ & $\begin{array}{c} \pm \\
(\%) \\
\end{array}$ \\
\hline 45 & $2-4-06-1-46$ & 193 & 9720 & 1.0 & 0.74818 & 4.3 \\
\hline 45 & $2-4-06-1-48$ & 179 & 7650 & 0.9 & 0.78364 & 6.3 \\
\hline 45 & $2-4-06-1-54$ & 94 & 6761 & 1.2 & 0.78652 & 2.6 \\
\hline 45 & $2-4-06-1-74$ & 46 & 4407 & 1.8 & 1.43173 & 3.1 \\
\hline 45 & $2-4-06-1-75$ & 53 & 6781 & 1.4 & 1.71618 & 2.9 \\
\hline 45 & $2-4-06-1-54$ & 288 & 21144 & 1.4 & 1.53413 & 5.6 \\
\hline 45 & $2-4-06-1-5$ & 65 & 8721 & 1.7 & 1.80630 & 4.2 \\
\hline 45 & $2-4-06-1-5$ & 156 & 17909 & 0.7 & 1.79553 & 3.2 \\
\hline 45 & $2-4-06-1-69$ & 81 & 8922 & 3.7 & 1.70586 & 4.8 \\
\hline 45 & $2-4-06-1-50$ & 76 & 10140 & 0.8 & 1.83321 & 2.5 \\
\hline 45 & $2-4-06-1-6$ & 102 & 14832 & 1.2 & 1.82021 & 3.5 \\
\hline 45 & $2-4-06-1-38$ & 134 & 12561 & 1.1 & 1.74064 & 2.7 \\
\hline 45 & $2-4-06-1-14$ & 211 & 35287 & 0.8 & 1.98922 & 3.2 \\
\hline 45 & $2-4-06-1-35$ & 71 & 10483 & 1.1 & 2.07548 & 2.7 \\
\hline 45 & $2-4-06-1-63$ & 160 & 25604 & 2.0 & 2.13250 & 2.0 \\
\hline 45 & $2-4-06-1-27$ & 27 & 3770 & 0.5 & 2.18134 & 4.9 \\
\hline 45 & $2-4-06-1-24$ & 97 & 13918 & 2.0 & 2.18223 & 2.5 \\
\hline 45 & $2-4-06-1-33$ & 531 & 38862 & 0.7 & 2.19570 & 1.5 \\
\hline 45 & $2-4-06-1-4$ & 114 & 14269 & 3.1 & 2.19126 & 1.7 \\
\hline 45 & $2-4-06-1-49$ & 115 & 20237 & 0.7 & 2.13326 & 4.9 \\
\hline 45 & $2-4-06-1-18$ & 32 & 3807 & 0.7 & 2.16283 & 4.1 \\
\hline 45 & $2-4-06-1-49$ & 249 & 20570 & 1.5 & 2.26053 & 2.4 \\
\hline 45 & $2-4-06-1-55$ & 328 & 4793 & 1.7 & 1.46234 & 7.0 \\
\hline 45 & $2-4-06-1-30$ & 59 & 6760 & 0.6 & 2.46876 & 3.5 \\
\hline 45 & $2-4-06-1-64$ & 257 & 45559 & 1.4 & 2.77348 & 1.7 \\
\hline 45 & $2-4-06-1-1$ & 701 & 8576 & 1.7 & 2.15333 & 8.3 \\
\hline 45 & 2-4-06-1-45 & 118 & 10101 & 2.9 & 2.29707 & 6.6 \\
\hline 45 & $2-4-06-1-23$ & 155 & 25760 & 0.4 & 3.12064 & 1.5 \\
\hline 45 & $2-4-06-1-26$ & 107 & 17290 & 1.1 & 3.09809 & 1.6 \\
\hline 45 & $2-4-06-1-71$ & 187 & 37580 & 1.9 & 3.46645 & 2.6 \\
\hline 45 & $2-4-06-1-8$ & 170 & 7075 & 2.6 & 2.57317 & 7.8 \\
\hline 45 & $2-4-06-1-61$ & 376 & 69392 & 0.5 & 3.43116 & 4.4 \\
\hline 45 & $2-4-06-1-40$ & 245 & 15279 & 0.8 & 3.06750 & 2.1 \\
\hline 45 & $2-4-06-1-20$ & 495 & 32124 & 1.0 & 4.11453 & 3.2 \\
\hline 45 & $2-4-06-1-44$ & 335 & 26277 & 0.9 & 4.17099 & 4.2 \\
\hline 45 & $2-4-06-1-33$ & 184 & 25048 & 1.7 & 3.88873 & 2.6 \\
\hline 45 & $2-4-06-1-65$ & 1310 & 30462 & 5.4 & 3.76252 & 3.5 \\
\hline 45 & $2-4-06-1-63$ & 23 & 5171 & 0.2 & 4.28497 & 5.0 \\
\hline 45 & $2-4-06-1-51$ & 450 & 60484 & 2.2 & 4.01481 & 2.9 \\
\hline 45 & $2-4-06-1-26$ & 372 & 45830 & 4.7 & 3.80422 & 2.0 \\
\hline 45 & $2-4-06-1-32$ & 409 & 92847 & 2.5 & 4.39977 & 2.9 \\
\hline 45 & $2-4-06-1-28$ & 304 & 29143 & 0.8 & 4.10922 & 3.3 \\
\hline 45 & $2-4-06-1-57$ & 407 & 66034 & 1.6 & 3.65638 & 1.7 \\
\hline 45 & $2-4-06-1-62$ & 46 & 7493 & 0.5 & 3.94281 & 2.3 \\
\hline 45 & $2-4-06-1-28$ & 138 & 28922 & 1.2 & 4.16308 & 1.8 \\
\hline 45 & $2-4-06-1-41$ & 166 & 13708 & 2.0 & 3.88868 & 7.0 \\
\hline 45 & $2-4-06-1-72$ & 155 & 18760 & 1.6 & 4.32865 & 2.1 \\
\hline 45 & $2-4-06-1-59$ & 291 & 34122 & 2.0 & 3.90639 & 3.2 \\
\hline 45 & $2-4-06-1-11$ & 249 & 48421 & 1.1 & 4.45573 & 2.3 \\
\hline 45 & $2-4-06-1-19$ & 172 & 21498 & 2.4 & 4.27167 & 3.3 \\
\hline 45 & $2-4-06-1-9$ & 160 & 32695 & 0.8 & 4.28283 & 2.7 \\
\hline 45 & $2-4-06-1-58$ & 92 & 7165 & 1.2 & 4.38988 & 4.4 \\
\hline 45 & $2-4-06-1-53$ & 101 & 6984 & 1.2 & 4.23230 & 3.5 \\
\hline 45 & $2-4-06-1-36$ & 56 & 3495 & 0.6 & 4.30378 & 4.5 \\
\hline 45 & $2-4-06-1-44$ & 306 & 28279 & 3.7 & 3.89996 & 7.9 \\
\hline 45 & $2-4-06-1-20$ & 324 & 21410 & 3.2 & 4.19036 & 9.6 \\
\hline 45 & $2-4-06-1-70$ & 36 & 10521 & 3.9 & 4.24017 & 3.9 \\
\hline 45 & $2-4-06-1-60$ & 507 & 42766 & 1.6 & 4.35385 & 4.4 \\
\hline 45 & $2-4-06-1-17$ & 101 & 24027 & 0.6 & 4.81063 & 4.2 \\
\hline 45 & 2-4-06-1-1 & 221 & 38270 & 1.3 & 5.16906 & 3.3 \\
\hline 45 & $2-4-06-1-3$ & 50 & 10479 & 0.8 & 5.55694 & 4.0 \\
\hline 45 & $2-4-06-1-10$ & 68 & 17660 & 0.7 & 5.70893 & 4.0 \\
\hline 45 & $2-4-06-1-16$ & 128 & 26852 & 0.6 & 5.72661 & 3.0 \\
\hline 45 & $2-4-06-1-34$ & 69 & 16162 & 1.5 & 6.15241 & 2.9 \\
\hline 45 & $2-4-06-1-43$ & 183 & 19274 & 0.5 & 5.87055 & 3.0 \\
\hline 45 & $2-4-06-1-8$ & 83 & 29173 & 0.5 & 7.01916 & 4.0 \\
\hline 45 & $2-4-06-1-27$ & 1042 & 16342 & 0.7 & 6.16314 & 3.3 \\
\hline 45 & $2-4-06-1-50$ & 160 & 36820 & 1.4 & 10.55794 & 1.7 \\
\hline 45 & $2-4-06-1-29$ & 69 & 21931 & 0.9 & 10.45470 & 2.5 \\
\hline 45 & $2-4-06-1-36$ & 267 & 17813 & 0.7 & 11.74949 & 7.5 \\
\hline 45 & $2-4-06-1-52$ & 95 & 15850 & 1.2 & 10.14967 & 3.1 \\
\hline 45 & $2-4-06-1-17$ & 183 & 57851 & 0.8 & 12.55167 & 1.9 \\
\hline 45 & $2-4-06-1-7$ & 85 & 29160 & 0.5 & 13.59448 & 3.9 \\
\hline 46 & $2-4-06-2-16$ & 2077 & 3923 & 2.4 & 0.03924 & 7.2 \\
\hline 46 & $2-4-06-2-32$ & 728 & 993 & 1.7 & 0.04950 & 5.0 \\
\hline 46 & $2-4-06-2-101$ & 1929 & 6539 & 7.0 & 0.05336 & 3.9 \\
\hline 46 & $2-4-06-2-89$ & 362 & 796 & 8.6 & 0.07666 & 11.0 \\
\hline 46 & $2-4-06-2-10$ & 625 & 2846 & 1.1 & 0.09522 & 11.2 \\
\hline 46 & $2-4-06-2-43$ & 446 & 2074 & 2.7 & 0.07479 & 19.9 \\
\hline 46 & $2-4-06-2-61$ & 415 & 2250 & 1.2 & 0.08404 & 9.1 \\
\hline 46 & $2-4-06-2-15$ & 523 & 4417 & 0.8 & 0.10850 & 9.9 \\
\hline 46 & $2-4-06-2-90$ & 544 & 3179 & 1.0 & 0.10458 & 4.3 \\
\hline 46 & $2-4-06-2-9$ & 769 & 3077 & 5.1 & 0.11575 & 7.5 \\
\hline 46 & $2-4-06-2-111$ & 1001 & 4943 & 3.2 & 0.14191 & 5.0 \\
\hline 46 & $2-4-06-2-16$ & 150 & 957 & 0.5 & 0.26651 & 33.1 \\
\hline 46 & $2-4-06-2-34$ & 880 & 1968 & 1.6 & 0.17721 & 4.4 \\
\hline 46 & $2-4-06-2-69$ & 596 & 5785 & 1.5 & 0.16798 & 4.4 \\
\hline 46 & $2-4-06-2-56$ & 212 & 1344 & 1.6 & 0.19533 & 7.7 \\
\hline 46 & $2-4-06-2-103$ & 630 & 7066 & 1.8 & 0.18296 & 4.5 \\
\hline 46 & $2-4-06-2-99$ & 497 & 1344 & 3.0 & 0.18843 & 5.6 \\
\hline
\end{tabular}


Kimbrough, D.L., Grove, M., Gehrels, G.E., Dorsey, R.J., Howard, K.A., Lovera, O., Aslan, A., House, P.K., and Pearthree, P.A., 2015, Detrital zircon U-Pb provenance of the Colorado River: A 5 m.y. record of incision into cover strata overlying the Colorado Plateau and adjacent regions: Geosphere, v. 11, doi:10.1130/GES00982.1.

TABLE DR7. U-Pb ZIRCON LA-ICP-MS GEOCHRONOLOGIC ANALYSES OF COLORADO RIVER BASIN \& ASSOCIATED SAMPLES.

\begin{tabular}{|c|c|c|c|c|c|c|}
\hline & & & & & & opic ra \\
\hline ID\# & * Analysis & $\begin{array}{c}U \\
(\mathrm{ppm})\end{array}$ & $\begin{array}{l}206 \mathrm{~Pb} \\
204 \mathrm{~Pb} \\
\end{array}$ & U/Th & $\begin{array}{c}207 \mathrm{~Pb}^{*} \\
235 \mathrm{U}\end{array}$ & $\begin{array}{c} \pm \\
(\%) \\
\end{array}$ \\
\hline 46 & $2-4-06-2-17$ & 672 & 3410 & 1.4 & 0.19723 & 4.8 \\
\hline 46 & 2-4-06-2-92 & 371 & 3420 & 1.3 & 0.23397 & 7.1 \\
\hline 46 & $2-4-06-2-4$ & 482 & 2878 & 3.6 & 0.26897 & 7.7 \\
\hline 46 & $2-4-06-2-66$ & 364 & 8291 & 1.8 & 0.26354 & 3.5 \\
\hline 46 & $2-4-06-2-78$ & 709 & 847 & 1.3 & 0.50802 & 5.4 \\
\hline 46 & 2-4-06-2-98 & 216 & 2094 & 1.9 & 0.43042 & 8.4 \\
\hline 46 & $2-4-06-2-31$ & 547 & 7742 & 4.1 & 0.45352 & 2.8 \\
\hline 46 & $2-4-06-2-8$ & 1033 & 29401 & 19.0 & 0.43811 & 3.0 \\
\hline 46 & $2-4-06-2-46$ & 889 & 3067 & 4.9 & 0.46304 & 4.8 \\
\hline 46 & $2-4-06-2-37$ & 535 & 4677 & 1.2 & 0.47941 & 4.7 \\
\hline 46 & $2-4-06-2-87$ & 175 & 4114 & 1.7 & 0.53191 & 4.9 \\
\hline 46 & 2-4-06-2-105 & 441 & 8701 & 1.9 & 0.52211 & 2.2 \\
\hline 46 & $2-4-06-2-6$ & 310 & 2315 & 1.9 & 0.53883 & 2.6 \\
\hline 46 & $2-4-06-2-70$ & 140 & 4745 & 2.1 & 0.59584 & 6.2 \\
\hline 46 & $2-4-06-2-42$ & 306 & 8417 & 1.7 & 0.55402 & 3.3 \\
\hline 46 & 2-4-06-2-113 & 794 & 12365 & 7.5 & 0.59345 & 5.7 \\
\hline 46 & $2-4-06-2-72$ & 399 & 2164 & 3.5 & 0.62160 & 3.7 \\
\hline 46 & $2-4-06-2-27$ & 625 & 22341 & 9.2 & 0.58650 & 4.4 \\
\hline 46 & $2-4-06-2-88$ & 532 & 12385 & 1.6 & 0.63107 & 6.8 \\
\hline 46 & $2-4-06-2-12$ & 247 & 2742 & 0.5 & 0.85994 & 9.2 \\
\hline 46 & $2-4-06-2-93$ & 560 & 15123 & 2.1 & 0.67682 & 2.5 \\
\hline 46 & $2-4-06-2-23$ & 318 & 7786 & 3.8 & 0.67399 & 6.7 \\
\hline 46 & $2-4-06-2-80$ & 392 & 7356 & 6.1 & 0.67761 & 7.0 \\
\hline 46 & $2-4-06-2-36$ & 378 & 3768 & 1.7 & 0.74356 & 5.5 \\
\hline 46 & $2-4-06-2-11$ & 201 & 6997 & 2.0 & 0.74402 & 3.7 \\
\hline 46 & $2-4-06-2-21$ & 196 & 5106 & 2.7 & 0.73131 & 3.2 \\
\hline 46 & $2-4-06-2-38$ & 541 & 5783 & 2.5 & 0.77208 & 3.6 \\
\hline 46 & $2-4-06-2-47$ & 237 & 4849 & 1.0 & 0.77079 & 7.4 \\
\hline 46 & $2-4-06-2-97$ & 77 & 2034 & 2.9 & 0.85090 & 12.3 \\
\hline 46 & 2-4-06-2-29 & 244 & 9344 & 3.0 & 0.75223 & 6.7 \\
\hline 46 & $2-4-06-2-25$ & 458 & 8215 & 6.7 & 0.85014 & 4.4 \\
\hline 46 & 2-4-06-2-114 & 696 & 2737 & 4.2 & 0.84819 & 3.7 \\
\hline 46 & 2-4-06-2-109 & 328 & 5982 & 4.4 & 0.87139 & 2.5 \\
\hline 46 & $2-4-06-2-68$ & 190 & 2176 & 0.6 & 1.47670 & 6.9 \\
\hline 46 & $2-4-06-2-1$ & 268 & 15135 & 3.7 & 1.54220 & 2.2 \\
\hline 46 & $2-4-06-2-82$ & 235 & 3835 & 2.3 & 1.53360 & 3.0 \\
\hline 46 & $2-4-06-2-44$ & 774 & 38460 & 3.5 & 1.56876 & 5.5 \\
\hline 46 & 2-4-06-2-35 & 396 & 6735 & 3.9 & 1.53328 & 2.4 \\
\hline 46 & $2-4-06-2-13$ & 283 & 41057 & 2.1 & 1.83904 & 2.5 \\
\hline 46 & $2-4-06-2-2$ & 450 & 25140 & 1.2 & 1.63060 & 6.9 \\
\hline 46 & $2-4-06-2-48$ & 892 & 3041 & 2.2 & 1.63630 & 6.3 \\
\hline 46 & $2-4-06-2-12$ & 503 & 3844 & 2.8 & 1.98544 & 4.1 \\
\hline 46 & $2-4-06-2-62$ & 624 & 32162 & 3.1 & 1.86738 & 1.5 \\
\hline 46 & $2-4-06-2-75$ & 341 & 22740 & 1.6 & 1.76051 & 3.5 \\
\hline 46 & $2-4-06-2-3$ & 114 & 10458 & 8.8 & 1.91357 & 2.3 \\
\hline 46 & $2-4-06-2-24$ & 181 & 9400 & 3.8 & 1.82936 & 3.8 \\
\hline 46 & $2-4-06-2-30$ & 598 & 11561 & 2.3 & 1.88683 & 2.8 \\
\hline 46 & $2-4-06-2-14$ & 270 & 26308 & 5.0 & 1.91698 & 2.0 \\
\hline 46 & $2-4-06-2-20$ & 113 & 11772 & 1.4 & 1.88347 & 3.8 \\
\hline 46 & $2-4-06-2-19$ & 1157 & 68618 & 10.7 & 1.86153 & 3.3 \\
\hline 46 & $2-4-06-2-73$ & 529 & 27704 & 5.3 & 1.86729 & 4.4 \\
\hline 46 & 2-4-06-2-95 & 99 & 7428 & 3.0 & 2.01461 & 3.6 \\
\hline 46 & $2-4-06-2-64$ & 158 & 8547 & 0.7 & 2.01271 & 4.8 \\
\hline 46 & $2-4-06-2-49$ & 573 & 21985 & 3.2 & 2.20145 & 2.2 \\
\hline 46 & $2-4-06-2-58$ & 637 & 3931 & 4.0 & 2.21756 & 3.7 \\
\hline 46 & $2-4-06-2-15$ & 437 & 12323 & 4.3 & 2.68306 & 2.7 \\
\hline 46 & 2-4-06-2-39 & 661 & 57302 & 2.2 & 2.76831 & 3.6 \\
\hline 46 & $2-4-06-2-7$ & 371 & 29455 & 2.5 & 2.80406 & 4.1 \\
\hline 46 & $2-4-06-2-57$ & 572 & 63688 & 2.1 & 2.84142 & 2.0 \\
\hline 46 & $2-4-06-2-108$ & 245 & 16165 & 3.1 & 2.59755 & 3.1 \\
\hline 46 & $2-4-06-2-5$ & 232 & 26610 & 2.0 & 2.83176 & 3.5 \\
\hline 46 & $2-4-06-2-107$ & 379 & 40385 & 1.8 & 2.83592 & 3.8 \\
\hline 46 & $2-4-06-2-63$ & 1087 & 12495 & 1.8 & 3.01810 & 2.8 \\
\hline 46 & $2-4-06-2-41$ & 537 & 34724 & 3.2 & 2.65433 & 2.9 \\
\hline 46 & $2-4-06-2-51$ & 66 & 5070 & 1.7 & 2.98037 & 6.2 \\
\hline 46 & $2-4-06-2-9$ & 129 & 28498 & 1.2 & 3.23916 & 2.4 \\
\hline 46 & $2-4-06-2-18$ & 247 & 3520 & 3.7 & 2.91336 & 3.0 \\
\hline 46 & $2-4-06-2-110$ & 304 & 33344 & 6.8 & 3.00942 & 3.1 \\
\hline 46 & $2-4-06-2-100$ & 196 & 11660 & 2.8 & 3.08303 & 3.7 \\
\hline 46 & $2-4-06-2-22$ & 422 & 54315 & 4.5 & 3.32437 & 4.4 \\
\hline 46 & $2-4-06-2-86$ & 534 & 10383 & 3.2 & 3.23646 & 3.3 \\
\hline 46 & $2-4-06-2-76$ & 425 & 59830 & 4.4 & 3.56751 & 3.3 \\
\hline 46 & $2-4-06-2-55$ & 66 & 10817 & 1.6 & 3.54425 & 5.5 \\
\hline 46 & $2-4-06-2-71$ & 300 & 26571 & 3.0 & 3.60839 & 3.7 \\
\hline 46 & $2-4-06-2-40$ & 269 & 5517 & 5.4 & 3.46049 & 5.9 \\
\hline 46 & $2-4-06-2-50$ & 250 & 29258 & 2.4 & 3.99907 & 2.9 \\
\hline 46 & $2-4-06-2-28$ & 416 & 15126 & 2.8 & 3.69180 & 4.5 \\
\hline 46 & $2-4-06-2-2$ & 237 & 28323 & 3.0 & 3.85966 & 4.2 \\
\hline 46 & $2-4-06-2-102$ & 166 & 9748 & 4.2 & 2.88661 & 4.5 \\
\hline 46 & $2-4-06-2-106$ & 437 & 10586 & 2.8 & 3.88927 & 2.3 \\
\hline 46 & $2-4-06-2-112$ & 228 & 13234 & 1.5 & 3.91320 & 4.0 \\
\hline 46 & $2-4-06-2-33$ & 349 & 3498 & 7.6 & 4.05428 & 3.8 \\
\hline 46 & $2-4-06-2-54$ & 143 & 2145 & 0.6 & 3.61984 & 5.5 \\
\hline 46 & $2-4-06-2-94$ & 988 & 25239 & 3.2 & 3.70657 & 4.8 \\
\hline 46 & $2-4-06-2-84$ & 292 & 23217 & 2.7 & 3.90537 & 2.7 \\
\hline 46 & $2-4-06-2-52$ & 273 & 32146 & 2.8 & 3.70974 & 5.6 \\
\hline 46 & $2-4-06-2-10$ & 135 & 10812 & 0.9 & 3.94676 & 3.2 \\
\hline 46 & $2-4-06-2-104$ & 636 & 46906 & 2.2 & 4.11159 & 3.6 \\
\hline 46 & $2-4-06-2-20$ & 657 & 13387 & 1.7 & 4.33402 & 4.1 \\
\hline 46 & $2-4-06-2-60$ & 451 & 59617 & 2.2 & 4.38643 & 3.3 \\
\hline
\end{tabular}


Kimbrough, D.L., Grove, M., Gehrels, G.E., Dorsey, R.J., Howard, K.A., Lovera, O., Aslan, A., House, P.K., and Pearthree, P.A., 2015, Detrital zircon U-Pb provenance of the Colorado River: A 5 m.y. record of incision into cover strata overlying the Colorado Plateau and adjacent regions: Geosphere, v. 11, doi:10.1130/GES00982.1.

TABLE DR7. U-Pb ZIRCON LA-ICP-MS GEOCHRONOLOGIC ANALYSES OF COLORADO RIVER BASIN \& ASSOCIATED SAMPLES.

\begin{tabular}{|c|c|c|c|c|c|c|}
\hline & & & & & & opic ra \\
\hline ID\# & Analysis & $\begin{array}{c}U \\
\text { (ppm) }\end{array}$ & $\begin{array}{l}206 \mathrm{~Pb} \\
204 \mathrm{~Pb}\end{array}$ & U/Th & $\begin{array}{c}207 \mathrm{~Pb}^{*} \\
235 \mathrm{U}\end{array}$ & $\begin{array}{c} \pm \\
(\%) \\
\end{array}$ \\
\hline 46 & $2-4-06-2-6$ & 274 & 35084 & 1.9 & 4.32784 & 7.5 \\
\hline 46 & $2-4-06-2-7$ & 560 & 93464 & 1.6 & 4.73897 & 2.6 \\
\hline 46 & $2-4-06-2-59$ & 426 & 43293 & 1.3 & 4.74276 & 4.4 \\
\hline 46 & $2-4-06-2-77$ & 190 & 37158 & 2.3 & 4.70532 & 2.3 \\
\hline 46 & $2-4-06-2-115$ & 139 & 8337 & 1.0 & 4.25632 & 5.7 \\
\hline 46 & $2-4-06-2-11$ & 317 & 32620 & 1.6 & 4.33495 & 10.2 \\
\hline 46 & $2-4-06-2-83$ & 837 & 5436 & 4.0 & 3.62388 & 4.4 \\
\hline 46 & $2-4-06-2-65$ & 481 & 3610 & 1.7 & 4.38547 & 5.1 \\
\hline 46 & $2-4-06-2-81$ & 458 & 31898 & 13.0 & 4.82060 & 4.5 \\
\hline 46 & $2-4-06-2-19$ & 107 & 35721 & 2.2 & 12.86426 & 5.1 \\
\hline 47 & $2-4-06-3-30$ & 144 & 465 & 0.6 & 0.01806 & 66.9 \\
\hline 47 & $2-4-06-3-18$ & 555 & 930 & 0.3 & 0.05503 & 21.5 \\
\hline 47 & 2-4-06-3-45 & 510 & 487 & 0.4 & 0.10577 & 31.3 \\
\hline 47 & $2-4-06-3-38$ & 1013 & 3511 & 11.8 & 0.08792 & 5.9 \\
\hline 47 & $2-4-06-3-7$ & 561 & 1418 & 2.6 & 0.10153 & 11.0 \\
\hline 47 & $2-4-06-3-61$ & 2819 & 5162 & 0.7 & 0.08699 & 2.6 \\
\hline 47 & $2-4-06-3-29$ & 535 & 2313 & 0.8 & 0.08567 & 7.9 \\
\hline 47 & $2-4-06-3-43$ & 708 & 2918 & 2.2 & 0.09430 & 7.3 \\
\hline 47 & $2-4-06-3-22$ & 445 & 3002 & 4.3 & 0.10225 & 6.6 \\
\hline 47 & $2-4-06-3-8$ & 859 & 7346 & 1.0 & 0.09708 & 3.8 \\
\hline 47 & 2-4-06-3-25 & 459 & 3928 & 2.9 & 0.10493 & 4.5 \\
\hline 47 & $2-4-06-3-27$ & 320 & 1759 & 1.4 & 0.15935 & 24.8 \\
\hline 47 & $2-4-06-3-20$ & 535 & 2574 & 4.3 & 0.18180 & 9.6 \\
\hline 47 & $2-4-06-3-3$ & 165 & 515 & 0.8 & 0.28608 & 6.5 \\
\hline 47 & $2-4-06-3-50$ & 344 & 5213 & 1.5 & 0.16546 & 7.0 \\
\hline 47 & $2-4-06-3-18$ & 350 & 2463 & 2.7 & 0.18502 & 3.7 \\
\hline 47 & 2-4-06-3-6 & 333 & 4409 & 2.1 & 0.19912 & 5.5 \\
\hline 47 & $2-4-06-3-25$ & 129 & 1400 & 1.2 & 0.26577 & 8.7 \\
\hline 47 & $2-4-06-3-46$ & 606 & 13137 & 1.3 & 0.19747 & 5.7 \\
\hline 47 & 2-4-06-3-15 & 522 & 11599 & 1.7 & 0.24543 & 4.3 \\
\hline 47 & $2-4-06-3-3$ & 420 & 1613 & 3.9 & 0.26711 & 10.8 \\
\hline 47 & $2-4-06-3-76$ & 253 & 2671 & 3.2 & 0.25870 & 4.4 \\
\hline 47 & $2-4-06-3-28$ & 1375 & 871 & 0.9 & 0.52184 & 7.0 \\
\hline 47 & $2-4-06-3-42$ & 262 & 1356 & 1.1 & 0.32018 & 12.5 \\
\hline 47 & $2-4-06-3-67$ & 1081 & 19918 & 2.1 & 0.28942 & 3.4 \\
\hline 47 & $2-4-06-3-74$ & 324 & 1042 & 0.9 & 0.35223 & 9.9 \\
\hline 47 & $2-4-06-3-16$ & 186 & 6170 & 2.3 & 0.33640 & 5.5 \\
\hline 47 & $2-4-06-3-23$ & 761 & 6514 & 2.9 & 0.34959 & 2.4 \\
\hline 47 & $2-4-06-3-56$ & 676 & 3559 & 3.0 & 0.38359 & 3.0 \\
\hline 47 & $2-4-06-3-77$ & 191 & 3081 & 3.9 & 0.43048 & 9.6 \\
\hline 47 & $2-4-06-3-56$ & 569 & 10955 & 1.5 & 0.41545 & 2.6 \\
\hline 47 & $2-4-06-3-11$ & 696 & 10804 & 4.4 & 0.48333 & 4.7 \\
\hline 47 & $2-4-06-3-33$ & 256 & 832 & 1.4 & 0.53550 & 11.7 \\
\hline 47 & $2-4-06-3-57$ & 354 & 4359 & 1.2 & 0.49268 & 3.9 \\
\hline 47 & $2-4-06-3-40$ & 574 & 6367 & 2.2 & 0.54033 & 6.1 \\
\hline 47 & $2-4-06-3-38$ & 132 & 6132 & 1.0 & 0.51277 & 4.7 \\
\hline 47 & $2-4-06-3-9$ & 254 & 4778 & 3.5 & 0.56646 & 4.9 \\
\hline 47 & $2-4-06-3-35$ & 233 & 6298 & 3.1 & 0.53055 & 3.6 \\
\hline 47 & $2-4-06-3-54$ & 468 & 9288 & 1.6 & 0.69792 & 3.5 \\
\hline 47 & $2-4-06-3-64$ & 144 & 812 & 1.0 & 0.68987 & 8.4 \\
\hline 47 & $2-4-06-3-44$ & 233 & 2268 & 1.4 & 0.74524 & 3.9 \\
\hline 47 & $2-4-06-3-48$ & 168 & 1057 & 0.6 & 0.64327 & 13.1 \\
\hline 47 & $2-4-06-3-49$ & 43 & 2098 & 154.5 & 0.83129 & 8.0 \\
\hline 47 & $2-4-06-3-72$ & 115 & 5229 & 1.1 & 0.77761 & 4.7 \\
\hline 47 & $2-4-06-3-4$ & 727 & 6787 & 4.2 & 0.87435 & 5.0 \\
\hline 47 & $2-4-06-3-11$ & 135 & 9664 & 0.5 & 0.93468 & 3.6 \\
\hline 47 & $2-4-06-3-31$ & 404 & 21942 & 4.0 & 1.10348 & 4.7 \\
\hline 47 & $2-4-06-3-52$ & 172 & 662 & 1.1 & 1.57642 & 4.6 \\
\hline 47 & $2-4-06-3-52$ & 179 & 17597 & 1.7 & 1.59942 & 3.1 \\
\hline 47 & $2-4-06-3-44$ & 327 & 29732 & 2.3 & 1.64475 & 1.8 \\
\hline 47 & 2-4-06-3-1 & 1236 & 88228 & 20.4 & 1.75611 & 2.4 \\
\hline 47 & $2-4-06-3-36$ & 131 & 19130 & 2.7 & 1.86534 & 2.0 \\
\hline 47 & $2-4-06-3-45$ & 698 & 3204 & 3.4 & 1.53979 & 3.7 \\
\hline 47 & $2-4-06-3-1$ & 132 & 15432 & 2.7 & 1.79935 & 5.3 \\
\hline 47 & 2-4-06-3-9 & 417 & 32399 & 1.0 & 1.57377 & 4.1 \\
\hline 47 & $2-4-06-3-21$ & 366 & 20593 & 1.6 & 1.68573 & 2.5 \\
\hline 47 & 2-4-06-3-6 & 66 & 6427 & 0.6 & 1.79625 & 3.6 \\
\hline 47 & $2-4-06-3-75$ & 350 & 70001 & 3.2 & 1.98500 & 2.3 \\
\hline 47 & $2-4-06-3-48$ & 119 & 6820 & 2.7 & 1.85073 & 4.4 \\
\hline 47 & $2-4-06-3-5$ & 104 & 8419 & 6.0 & 1.90159 & 5.2 \\
\hline 47 & $2-4-06-3-32$ & 333 & 13625 & 6.9 & 1.86999 & 4.3 \\
\hline 47 & $2-4-06-3-41$ & 827 & 73240 & 6.6 & 2.09376 & 3.5 \\
\hline 47 & $2-4-06-3-37$ & 234 & 15381 & 4.4 & 2.18290 & 3.5 \\
\hline 47 & $2-4-06-3-12$ & 193 & 18510 & 3.7 & 2.16281 & 4.2 \\
\hline 47 & $2-4-06-3-19$ & 234 & 20854 & 2.3 & 2.26686 & 4.6 \\
\hline 47 & $2-4-06-3-70$ & 144 & 10070 & 1.1 & 2.14046 & 2.2 \\
\hline 47 & $2-4-06-3-33$ & 200 & 18669 & 1.9 & 2.15073 & 4.1 \\
\hline 47 & $2-4-06-3-42$ & 507 & 37815 & 2.5 & 2.73385 & 2.7 \\
\hline 47 & $2-4-06-3-13$ & 356 & 43544 & 2.4 & 3.09878 & 3.9 \\
\hline 47 & $2-4-06-3-68$ & 189 & 5139 & 1.9 & 2.83886 & 2.4 \\
\hline 47 & $2-4-06-3-7$ & 95 & 11370 & 1.9 & 2.98987 & 1.6 \\
\hline 47 & $2-4-06-3-57$ & 190 & 26332 & 1.1 & 2.79057 & 3.1 \\
\hline 47 & $2-4-06-3-37$ & 176 & 38907 & 2.7 & 3.19179 & 3.4 \\
\hline 47 & $2-4-06-3-36$ & 170 & 6728 & 2.0 & 3.18326 & 2.3 \\
\hline 47 & $2-4-06-3-79$ & 210 & 346 & 3.7 & 2.07527 & 10.1 \\
\hline 47 & $2-4-06-3-59$ & 335 & 42652 & 2.3 & 3.30496 & 2.5 \\
\hline 47 & $2-4-06-3-73$ & 213 & 13147 & 2.8 & 3.44748 & 1.7 \\
\hline 47 & $2-4-06-3-27$ & 824 & 10838 & 3.7 & 2.83700 & 4.6 \\
\hline 47 & $2-4-06-3-62$ & 585 & 9298 & 1.3 & 3.20188 & 3.7 \\
\hline 47 & $2-4-06-3-17$ & 164 & 26139 & 1.5 & 3.67761 & 2.2 \\
\hline
\end{tabular}


Kimbrough, D.L., Grove, M., Gehrels, G.E., Dorsey, R.J., Howard, K.A., Lovera, O., Aslan, A., House, P.K., and Pearthree, P.A., 2015, Detrital zircon U-Pb provenance of the Colorado River: A 5 m.y. record of incision into cover strata overlying the Colorado Plateau and adjacent regions: Geosphere, v. 11, doi:10.1130/GES00982.1.

TABLE DR7. U-Pb ZIRCON LA-ICP-MS GEOCHRONOLOGIC ANALYSES OF COLORADO RIVER BASIN \& ASSOCIATED SAMPLES.

\begin{tabular}{|c|c|c|c|c|c|c|}
\hline & & & & & & topic rati \\
\hline ID\# & Analysis & $\begin{array}{c}\text { U } \\
(\mathrm{ppm})\end{array}$ & $\begin{array}{l}206 \mathrm{~Pb} \\
204 \mathrm{~Pb}\end{array}$ & $\mathrm{U} / \mathrm{Th}$ & $\begin{array}{c}207 \mathrm{~Pb}^{*} \\
235 \mathrm{U}\end{array}$ & $\begin{array}{c} \pm \\
(\%) \\
\end{array}$ \\
\hline & $2-4-06-3-19$ & 65 & 4553 & 77.4 & 3.37926 & 5.5 \\
\hline 47 & $2-4-06-3-53$ & 194 & 15399 & 3.9 & 3.77948 & 1.8 \\
\hline 47 & $2-4-06-3-2$ & 71 & 5374 & 3.9 & 3.50973 & 6.9 \\
\hline 47 & $2-4-06-3-32$ & 533 & 30466 & 12.6 & 3.03326 & 7.2 \\
\hline 47 & $2-4-06-3-55$ & 371 & 20521 & 1.1 & 4.02244 & 4.1 \\
\hline 47 & $2-4-06-3-21$ & 124 & 9621 & 1.4 & 4.15991 & 2.9 \\
\hline 47 & $2-4-06-3-71$ & 807 & 13387 & 0.4 & 4.29194 & 3.6 \\
\hline 47 & $2-4-06-3-54$ & 134 & 8534 & 1.7 & 3.72329 & 3.4 \\
\hline 47 & $2-4-06-3-58$ & 396 & 19222 & 2.3 & 3.89791 & 3.7 \\
\hline 47 & $2-4-06-3-58$ & 86 & 7799 & 0.4 & 3.59922 & 5.6 \\
\hline 47 & $2-4-06-3-60$ & 304 & 11427 & 4.5 & 3.58427 & 3.9 \\
\hline 47 & $2-4-06-3-22$ & 117 & 7812 & 1.1 & 3.53087 & 4.6 \\
\hline 47 & 2-4-06-3-59 & 513 & 13408 & 2.9 & 3.39320 & 5.7 \\
\hline 47 & $2-4-06-3-17$ & 228 & 36514 & 4.6 & 4.22847 & 2.1 \\
\hline 47 & $2-4-06-3-39$ & 176 & 21882 & 1.7 & 4.05082 & 3.9 \\
\hline 47 & $2-4-06-3-47$ & 425 & 35996 & 1.3 & 4.54842 & 4.2 \\
\hline 47 & $2-4-06-3-15$ & 381 & 20237 & 6.3 & 4.37727 & 5.6 \\
\hline 47 & $2-4-06-3-26$ & 220 & 16964 & 1.1 & 4.15860 & 6.3 \\
\hline 47 & $2-4-06-3-66$ & 179 & 29132 & 2.2 & 4.43942 & 2.6 \\
\hline 47 & $2-4-06-3-53$ & 586 & 106210 & 5.9 & 4.31822 & 3.0 \\
\hline 47 & 2-4-06-3-41 & 272 & 20107 & 1.4 & 4.51534 & 2.3 \\
\hline 47 & $2-4-06-3-46$ & 199 & 21035 & 1.9 & 4.39953 & 3.8 \\
\hline 47 & $2-4-06-3-78$ & 617 & 80938 & 9.1 & 4.12113 & 2.4 \\
\hline 47 & $2-4-06-3-69$ & 65 & 10442 & 1.0 & 4.36802 & 3.9 \\
\hline 47 & $2-4-06-3-55$ & 106 & 7888 & 1.4 & 4.05802 & 2.8 \\
\hline 47 & $2-4-06-3-24$ & 826 & 74796 & 32.4 & 4.61805 & 5.5 \\
\hline 47 & 2-4-06-3-65 & 315 & 15534 & 1.7 & 4.58725 & 2.7 \\
\hline 47 & $2-4-06-3-40$ & 232 & 11058 & 2.9 & 4.27066 & 7.4 \\
\hline 47 & $2-4-06-3-8$ & 208 & 4192 & 1.5 & 4.06267 & 5.3 \\
\hline 47 & $2-4-06-3-63$ & 607 & 3109 & 2.8 & 3.95292 & 4.1 \\
\hline 47 & $2-4-06-3-43$ & 399 & 9037 & 3.1 & 3.20523 & 6.3 \\
\hline 47 & $2-4-06-3-34$ & 265 & 11871 & 3.3 & 4.73962 & 2.7 \\
\hline 47 & $2-4-06-3-29$ & 370 & 8927 & 1.1 & 3.89711 & 2.5 \\
\hline 47 & $2-4-06-3-13$ & 291 & 34927 & 3.6 & 4.89660 & 2.6 \\
\hline 47 & $2-4-06-3-49$ & 219 & 14169 & 2.7 & 4.79194 & 3.8 \\
\hline 47 & $2-4-06-3-26$ & 311 & 9495 & 2.9 & 5.14887 & 3.4 \\
\hline 47 & $2-4-06-3-14$ & 278 & 4865 & 1.0 & 3.92764 & 5.5 \\
\hline 47 & $2-4-06-3-51$ & 72 & 6173 & 6.3 & 4.94566 & 4.1 \\
\hline 47 & $2-4-06-3-34$ & 93 & 20730 & 1.6 & 5.17317 & 4.7 \\
\hline 47 & $2-4-06-3-50$ & 294 & 30808 & 2.9 & 5.33847 & 2.9 \\
\hline 47 & $2-4-06-3-39$ & 458 & 46413 & 4.7 & 5.62566 & 2.4 \\
\hline 47 & $2-4-06-3-28$ & 314 & 2311 & 1.8 & 6.25642 & 3.6 \\
\hline 47 & $2-4-06-3-24$ & 469 & 13141 & 1.6 & 5.64271 & 8.9 \\
\hline 47 & $2-4-06-3-10$ & 189 & 2833 & 2.9 & 5.78988 & 8.1 \\
\hline 47 & $2-4-06-3-31$ & 97 & 11186 & 1.2 & 9.02139 & 3.5 \\
\hline 47 & $2-4-06-3-47$ & 108 & 20876 & 3.3 & 26.73997 & 2.4 \\
\hline 48 & TBP81412-4 & 186 & 5854 & 0.8 & 0.0270 & 28.5 \\
\hline 48 & TBP81412-64 & 127 & 1485 & 1.1 & 0.0341 & 264.3 \\
\hline 48 & TBP81412-46 & 1296 & 33858 & 0.5 & 0.0244 & 11.1 \\
\hline 48 & TBP81412-91 & 215 & 6148 & 1.2 & 0.0217 & 47.8 \\
\hline 48 & TBP81412-42 & 247 & 3899 & 1.0 & 0.0265 & 90.0 \\
\hline 48 & TBP81412-82 & 220 & 2182 & 1.3 & 0.0283 & 65.2 \\
\hline 48 & TBP81412-52 & 455 & 10541 & 0.8 & 0.0211 & 34.6 \\
\hline 48 & TBP81412-37 & 55 & 1004 & 1.2 & 0.1597 & 323.5 \\
\hline 48 & TBP81412-24 & 182 & 3377 & 2.2 & 0.0168 & 212.7 \\
\hline 48 & TBP81412-100 & 180 & 3546 & 0.8 & 0.0221 & 50.9 \\
\hline 48 & TBP81412-93 & 113 & 3034 & 1.6 & 0.0742 & 236.0 \\
\hline 48 & TBP81412-98 & 225 & 7893 & 0.9 & 0.0329 & 34.7 \\
\hline 48 & TBP81412-9 & 149 & 3107 & 0.9 & 0.0300 & 58.2 \\
\hline 48 & TBP81412-68 & 630 & 21531 & 0.6 & 0.0364 & 13.4 \\
\hline 48 & TBP81412-32 & 81 & 2465 & 0.8 & 0.0358 & 45.3 \\
\hline 48 & TBP81412-11 & 224 & 3500 & 2.6 & 0.0472 & 35.7 \\
\hline 48 & TBP81412-23 & 99 & 2109 & 0.8 & 0.3757 & 1155.8 \\
\hline 48 & TBP81412-28 & 80 & 1459 & 0.9 & 0.0446 & 78.2 \\
\hline 48 & TBP81412-38 & 54 & 1327 & 0.9 & 0.0709 & 179.4 \\
\hline 48 & TBP81412-99 & 230 & 3535 & 2.5 & 0.0406 & 19.4 \\
\hline 48 & TBP81412-63 & 270 & 6140 & 2.7 & 0.0424 & 27.0 \\
\hline 48 & TBP81412-66 & 312 & 12920 & 0.7 & 0.0599 & 20.5 \\
\hline 48 & TBP81412-3 & 857 & 30159 & 1.7 & 0.0606 & 4.4 \\
\hline 48 & TBP81412-39 & 40 & 3283 & 1.2 & 0.1276 & 77.2 \\
\hline 48 & TBP81412-62 & 139 & 4277 & 2.7 & 0.0689 & 23.1 \\
\hline 48 & TBP81412-29 & 102 & 3683 & 2.8 & 0.0698 & 26.4 \\
\hline 48 & TBP81412-85 & 416 & 18257 & 2.0 & 0.0848 & 12.7 \\
\hline 48 & TBP81412-87 & 68 & 12775 & 0.8 & 0.1596 & 22.9 \\
\hline 48 & TBP81412-13 & 64 & 5723 & 0.4 & 0.1615 & 14.5 \\
\hline 48 & TBP81412-56 & 437 & 39017 & 1.1 & 0.2192 & 3.3 \\
\hline 48 & TBP81412-34 & 264 & 34511 & 1.9 & 0.2495 & 4.4 \\
\hline 48 & TBP81412-60 & 229 & 41425 & 1.3 & 0.2603 & 4.4 \\
\hline 48 & TBP81412-33 & 223 & 36699 & 1.6 & 0.2504 & 6.3 \\
\hline 48 & TBP81412-89 & 265 & 76779 & 1.0 & 0.4011 & 3.6 \\
\hline 48 & TBP81412-6 & 106 & 40947 & 2.3 & 0.6621 & 5.5 \\
\hline 48 & TBP81412-77 & 39 & 19216 & 0.5 & 1.7490 & 4.4 \\
\hline 48 & TBP81412-58 & 206 & 102977 & 1.0 & 1.7701 & 1.4 \\
\hline 48 & TBP81412-26 & 50 & 36085 & 0.8 & 1.9269 & 2.8 \\
\hline 48 & TBP81412-41 & 79 & 39906 & 1.3 & 2.1789 & 2.4 \\
\hline 48 & TBP81412-75 & 37 & 50988 & 1.5 & 2.3322 & 2.5 \\
\hline 48 & TBP81412-25 & 81 & 103864 & 1.2 & 2.9511 & 1.2 \\
\hline 48 & TBP81412-17 & 42 & 34420 & 1.7 & 3.0168 & 3.0 \\
\hline 48 & TBP81412-27 & 34 & 25118 & 0.9 & 3.1687 & 3.9 \\
\hline 48 & TBP81412-65 & 58 & 53418 & 0.9 & 3.0774 & 2.5 \\
\hline
\end{tabular}


Kimbrough, D.L., Grove, M., Gehrels, G.E., Dorsey, R.J., Howard, K.A., Lovera, O., Aslan, A., House, P.K., and Pearthree, P.A., 2015, Detrital zircon U-Pb provenance of the Colorado River: A 5 m.y. record of incision into cover strata overlying the Colorado Plateau and adjacent regions: Geosphere, v. 11, doi:10.1130/GES00982.1.

TABLE DR7. U-Pb ZIRCON LA-ICP-MS GEOCHRONOLOGIC ANALYSES OF COLORADO RIVER BASIN \& ASSOCIATED SAMPLES.

\begin{tabular}{|c|c|c|c|c|c|c|}
\hline & & & & & & topic rat \\
\hline ID\# & $\neq$ Analysis & $\begin{array}{c}\text { U } \\
(\mathrm{ppm})\end{array}$ & $\begin{array}{l}206 \mathrm{~Pb} \\
204 \mathrm{~Pb}\end{array}$ & U/Th & $\begin{array}{c}207 \mathrm{~Pb}^{*} \\
235 \mathrm{U}\end{array}$ & $\begin{array}{c} \pm \\
(\%)\end{array}$ \\
\hline 48 & TBP81412-81 & 25 & 21711 & 0.4 & 3.0375 & 6.4 \\
\hline 48 & TBP81412-88 & 142 & 102633 & 0.8 & 3.0995 & 2.7 \\
\hline 48 & TBP81412-14 & 46 & 32778 & 1.7 & 3.1055 & 2.5 \\
\hline 48 & TBP81412-47 & 83 & 53858 & 0.6 & 3.1062 & 2.1 \\
\hline 48 & TBP81412-83 & 121 & 555518 & 1.0 & 3.1589 & 2.2 \\
\hline 48 & TBP81412-18 & 36 & 40359 & 0.6 & 3.0647 & 3.6 \\
\hline 48 & TBP81412-69 & 107 & 62106 & 1.2 & 3.1690 & 1.4 \\
\hline 48 & TBP81412-1 & 113 & 131746 & 1.6 & 3.1211 & 1.9 \\
\hline 48 & TBP81412-73 & 77 & 82592 & 1.0 & 3.1630 & 1.5 \\
\hline 48 & TBP81412-78 & 155 & 146127 & 1.3 & 3.1388 & 2.3 \\
\hline 48 & TBP81412-71 & 135 & 91421 & 1.6 & 3.1303 & 1.0 \\
\hline 48 & TBP81412-48 & 13 & 14349 & 1.1 & 3.1061 & 6.4 \\
\hline 48 & TBP81412-55 & 351 & 24254 & 0.6 & 3.0398 & 1.7 \\
\hline 48 & TBP81412-19 & 154 & 268433 & 0.7 & 3.1275 & 2.5 \\
\hline 48 & TBP81412-97 & 384 & 552130 & 31.1 & 3.1391 & 1.2 \\
\hline 48 & TBP81412-53 & 61 & 42006 & 1.1 & 3.1484 & 1.9 \\
\hline 48 & TBP81412-15 & 106 & 92171 & 1.5 & 3.1564 & 2.2 \\
\hline 48 & TBP81412-36 & 188 & 176822 & 1.4 & 3.2251 & 1.0 \\
\hline 48 & TBP81412-54 & 189 & 337228 & 1.7 & 3.1528 & 1.9 \\
\hline 48 & TBP81412-43 & 50 & 56959 & 1.0 & 3.2719 & 3.8 \\
\hline 48 & TBP81412-49 & 42 & 38553 & 1.1 & 3.1575 & 2.6 \\
\hline 48 & TBP81412-57 & 76 & 261677 & 0.8 & 3.1363 & 2.3 \\
\hline 48 & TBP81412-76 & 15 & 9558 & 0.6 & 3.1810 & 5.0 \\
\hline 48 & TBP81412-30 & 159 & 179930 & 2.0 & 3.3923 & 5.4 \\
\hline 48 & TBP81412-7 & 136 & 240356 & 1.5 & 4.1493 & 0.9 \\
\hline 48 & TBP81412-84 & 68 & 77741 & 0.9 & 4.2945 & 1.2 \\
\hline 48 & TBP81412-67 & 149 & 102579 & 1.9 & 4.0342 & 1.8 \\
\hline 48 & TBP81412-16 & 233 & 189556 & 2.6 & 4.2869 & 1.1 \\
\hline 48 & TBP81412-94 & 163 & 294585 & 2.3 & 4.3349 & 0.7 \\
\hline 48 & TBP81412-74 & 369 & 944424 & 2.0 & 4.3496 & 1.3 \\
\hline 48 & TBP81412-5 & 136 & 81017 & 1.6 & 4.3222 & 1.4 \\
\hline 48 & TBP81412-79 & 230 & 264363 & 2.2 & 4.3623 & 1.2 \\
\hline 48 & TBP81412-59 & 86 & 70387 & 2.0 & 4.3170 & 2.5 \\
\hline 48 & TBP81412-10 & 121 & 214627 & 1.7 & 4.4321 & 4.1 \\
\hline 48 & TBP81412-21 & 405 & 419925 & 10.6 & 4.4552 & 0.9 \\
\hline 48 & TBP81412-70 & 144 & 231535 & 1.2 & 4.3802 & 1.9 \\
\hline 48 & TBP81412-12 & 107 & 139445 & 1.7 & 4.3946 & 0.9 \\
\hline 48 & TBP81412-96 & 272 & 56762 & 1.7 & 4.4733 & 2.5 \\
\hline 48 & TBP81412-80 & 145 & 154511 & 1.7 & 4.4994 & 1.1 \\
\hline 48 & TBP81412-8 & 201 & 234727 & 0.8 & 4.4357 & 1.8 \\
\hline 48 & TBP81412-44 & 96 & 11493 & 1.1 & 4.2731 & 3.0 \\
\hline 48 & TBP81412-45 & 104 & 153792 & 1.5 & 4.4450 & 1.6 \\
\hline 48 & TBP81412-50 & 111 & 153548 & 1.4 & 4.5287 & 1.2 \\
\hline 48 & TBP81412-51 & 92 & 53897 & 2.5 & 4.5252 & 1.6 \\
\hline 48 & TBP81412-86 & 55 & 75092 & 1.4 & 4.5565 & 1.7 \\
\hline 48 & TBP81412-90 & 227 & 337676 & 2.1 & 4.6655 & 0.9 \\
\hline 48 & TBP81412-2 & 142 & 307821 & 2.2 & 4.5553 & 1.4 \\
\hline 48 & TBP81412-40 & 223 & 520215 & 2.1 & 12.0021 & 0.6 \\
\hline 48 & TBP81412-35 & 255 & 594356 & 1.6 & 12.7043 & 2.3 \\
\hline 48 & TBP81412-22 & 163 & 483346 & 2.7 & 13.1617 & 2.6 \\
\hline 48 & TBP81412-95 & 74 & 163760 & 0.8 & 13.3739 & 3.4 \\
\hline 49 & TBP79122-99 & 922 & 23564 & 0.9 & 0.0187 & 14.2 \\
\hline 49 & TBP79122-44 & 183 & 4234 & 1.2 & 0.0150 & 103.3 \\
\hline 49 & TBP79122-1 & 87 & 1625 & 0.8 & 0.0770 & 109.1 \\
\hline 49 & TBP79122-86 & 367 & 4275 & 0.4 & 0.0259 & 36.6 \\
\hline 49 & TBP79122-71 & 144 & 2287 & 1.3 & 0.0409 & 42.9 \\
\hline 49 & TBP79122-98 & 167 & 3193 & 1.0 & 0.0412 & 32.1 \\
\hline 49 & TBP79122-48 & 200 & 1848 & 1.9 & 0.0261 & 41.9 \\
\hline 49 & TBP79122-47 & 231 & 7324 & 1.4 & 0.0675 & 218.8 \\
\hline 49 & TBP79122-76 & 250 & 5338 & 1.2 & 0.0221 & 41.8 \\
\hline 49 & TBP79122-81 & 111 & 2038 & 1.0 & 0.0538 & 56.8 \\
\hline 49 & TBP79122-60 & 292 & 1456 & 0.6 & 0.0365 & 30.0 \\
\hline 49 & TBP79122-14 & 157 & 2480 & 0.6 & 0.0493 & 40.9 \\
\hline 49 & TBP79122-24 & 390 & 9499 & 0.8 & 0.0462 & 13.6 \\
\hline 49 & TBP79122-64 & 172 & 8541 & 1.4 & 0.0563 & 21.4 \\
\hline 49 & TBP79122-36 & 123 & 2806 & 0.8 & 0.0513 & 20.7 \\
\hline 49 & TBP79122-91 & 548 & 15931 & 1.0 & 0.0575 & 10.6 \\
\hline 49 & TBP79122-78 & 57 & 2436 & 1.3 & 0.2558 & 720.1 \\
\hline 49 & TBP79122-9 & 285 & 20358 & 1.5 & 0.0831 & 12.2 \\
\hline 49 & TBP79122-40 & 162 & 16260 & 1.6 & 0.1047 & 20.9 \\
\hline 49 & TBP79122-57 & 71 & 2509 & 0.5 & 0.1966 & 180.3 \\
\hline 49 & TBP79122-94 & 122 & 9023 & 1.5 & 0.1082 & 16.8 \\
\hline 49 & TBP79122-26 & 109 & 4727 & 0.9 & 0.1030 & 16.9 \\
\hline 49 & TBP79122-80 & 74 & 9781 & 1.3 & 0.1817 & 16.5 \\
\hline 49 & TBP79122-88 & 92 & 10040 & 0.6 & 0.1717 & 15.9 \\
\hline 49 & TBP79122-28 & 177 & 17467 & 0.9 & 0.1718 & 8.7 \\
\hline 49 & TBP79122-6 & 69 & 5081 & 1.2 & 0.1802 & 17.3 \\
\hline 49 & TBP79122-73 & 356 & 38391 & 2.5 & 0.1859 & 3.1 \\
\hline 49 & TBP79122-12 & 170 & 22908 & 1.0 & 0.1896 & 9.9 \\
\hline 49 & TBP79122-3 & 401 & 53565 & 2.0 & 0.2514 & 3.4 \\
\hline 49 & TBP79122-41 & 160 & 27379 & 2.2 & 0.2320 & 6.3 \\
\hline 49 & TBP79122-2 & 141 & 24166 & 1.8 & 0.2468 & 8.5 \\
\hline 49 & TBP79122-93 & 145 & 26849 & 1.7 & 0.2901 & 6.7 \\
\hline 49 & TBP79122-46 & 120 & 35241 & 1.1 & 0.4116 & 6.2 \\
\hline 49 & TBP79122-82 & 101 & 76144 & 1.5 & 1.7663 & 2.6 \\
\hline 49 & TBP79122-62 & 114 & 80914 & 2.0 & 1.6812 & 2.4 \\
\hline 49 & TBP79122-96 & 44 & 48443 & 0.8 & 1.7662 & 5.0 \\
\hline 49 & TBP79122-97 & 74 & 20646 & 2.8 & 1.8419 & 2.1 \\
\hline 49 & TBP79122-79 & 110 & 85567 & 1.7 & 1.8166 & 1.8 \\
\hline 49 & TBP79122-53 & 18 & 20732 & 0.9 & 2.0477 & 6.9 \\
\hline
\end{tabular}


Kimbrough, D.L., Grove, M., Gehrels, G.E., Dorsey, R.J., Howard, K.A., Lovera, O., Aslan, A., House, P.K., and Pearthree, P.A., 2015, Detrital zircon U-Pb provenance of the Colorado River: A 5 m.y. record of incision into cover strata overlying the Colorado Plateau and adjacent regions: Geosphere, v. 11, doi:10.1130/GES00982.1.

TABLE DR7. U-Pb ZIRCON LA-ICP-MS GEOCHRONOLOGIC ANALYSES OF COLORADO RIVER BASIN \& ASSOCIATED SAMPLES.

\begin{tabular}{|c|c|c|c|c|c|c|c|c|c|c|c|c|c|c|c|c|c|}
\hline \multirow[b]{2}{*}{ ID\# } & \multirow[b]{2}{*}{ \# Analysis } & \multirow[b]{2}{*}{$\underset{(\mathrm{ppm})}{U}$} & \multirow[b]{2}{*}{$\begin{array}{l}206 \mathrm{~Pb} \\
204 \mathrm{~Pb}\end{array}$} & \multirow[b]{2}{*}{$\mathrm{U} / \mathrm{Th}$} & \multicolumn{4}{|c|}{ Isotopic ratios } & \multirow[b]{2}{*}{$\begin{array}{l}\text { error } \\
\text { corr. }\end{array}$} & \multicolumn{6}{|c|}{ " Apparent ages (Ma) } & \multirow[b]{2}{*}{$\begin{array}{l}\text { Best age } \\
\text { (Ma) }\end{array}$} & \multirow[b]{2}{*}{$\begin{array}{c} \pm \\
(\mathrm{Ma})\end{array}$} \\
\hline & & & & & $\begin{array}{c}207 \mathrm{~Pb}^{*} \\
235 \mathrm{U}\end{array}$ & $\begin{array}{c} \pm \\
(\%)\end{array}$ & $\begin{array}{c}206 \mathrm{~Pb}^{*} \\
238 \mathrm{U}\end{array}$ & $\begin{array}{c} \pm \\
(\%)\end{array}$ & & $\begin{array}{c}206 \mathrm{~Pb}^{*} \\
238 \mathrm{U}\end{array}$ & $\begin{array}{c} \pm \\
(\mathrm{Ma})\end{array}$ & $\begin{array}{c}207 \mathrm{~Pb}^{\star} \\
235 \mathrm{U}\end{array}$ & $\begin{array}{c} \pm \\
(\mathrm{Ma})\end{array}$ & $\begin{array}{l}206 \mathrm{~Pb}^{*} \\
207 \mathrm{~Pb}^{\star}\end{array}$ & $\begin{array}{c} \pm \\
(\mathrm{Ma})\end{array}$ & & \\
\hline 49 & TBP79122-72 & 39 & 42096 & 2.5 & 2.0240 & 3.5 & 0.1927 & 0.9 & 0.25 & 1136.1 & 9.1 & 1123.6 & 24.1 & 1099.7 & 68.8 & 1099.7 & 68.8 \\
\hline 49 & TBP79122-17 & 58 & 65229 & 2.0 & 1.9472 & 2.4 & 0.1843 & 1.7 & 0.72 & 1090.6 & 17.3 & 1097.5 & 16.1 & 1111.3 & 33.5 & 1111.3 & 33.5 \\
\hline 49 & TBP79122-50 & 36 & 49267 & 2.1 & 2.0352 & 2.8 & 0.1907 & 1.4 & 0.49 & 1125.1 & 14.1 & 1127.4 & 19.0 & 1131.7 & 48.4 & 1131.7 & 48.4 \\
\hline 49 & TBP79122-54 & 136 & 117322 & 0.7 & 2.0834 & 1.8 & 0.1940 & 1.5 & 0.82 & 1143.1 & 15.6 & 1143.4 & 12.5 & 1143.9 & 20.7 & 1143.9 & 20.7 \\
\hline 49 & TBP79122-63 & 33 & 35897 & 1.2 & 2.1495 & 4.0 & 0.1984 & 3.0 & 0.75 & 1166.8 & 32.3 & 1164.9 & 28.0 & 1161.4 & 52.8 & 1161.4 & 52.8 \\
\hline 49 & TBP79122-33 & 105 & 96489 & 1.8 & 2.1849 & 2.2 & 0.2001 & 0.9 & 0.40 & 1175.9 & 9.2 & 1176.3 & 15.0 & 1176.8 & 39.1 & 1176.8 & 39.1 \\
\hline 49 & TBP79122-43 & 44 & 32259 & 3.0 & 2.6158 & 2.2 & 0.2234 & 1.2 & 0.54 & 1299.9 & 14.3 & 1305.1 & 16.5 & 1313.7 & 36.6 & 1313.7 & 36.6 \\
\hline 49 & TBP79122-34 & 89 & 101566 & 1.8 & 2.7189 & 2.0 & 0.2317 & 1.6 & 0.78 & 1343.5 & 18.9 & 1333.6 & 14.7 & 1317.8 & 23.9 & 1317.8 & 23.9 \\
\hline 49 & TBP79122-15 & 60 & 54477 & 1.2 & 2.9839 & 2.4 & 0.2438 & 1.8 & 0.73 & 1406.4 & 22.2 & 1403.5 & 18.4 & 1399.1 & 31.9 & 1399.1 & 31.9 \\
\hline 49 & TBP79122-10 & 189 & 103441 & 1.6 & 2.9528 & 1.2 & 0.2408 & 1.1 & 0.90 & 1391.0 & 13.4 & 1395.6 & 9.0 & 1402.5 & 10.0 & 1402.5 & 10.0 \\
\hline 49 & TBP79122-20 & 141 & 113589 & 0.9 & 2.8329 & 1.8 & 0.2303 & 1.7 & 0.95 & 1336.0 & 20.7 & 1364.3 & 13.5 & 1408.9 & 10.7 & 1408.9 & 10.7 \\
\hline 49 & TBP79122-16 & 45 & 48204 & 1.0 & 3.0476 & 2.1 & 0.2471 & 1.7 & 0.78 & 1423.3 & 21.2 & 1419.6 & 16.2 & 1414.0 & 25.1 & 1414.0 & 25.1 \\
\hline 49 & TBP79122-18 & 139 & 97268 & 1.9 & 2.9555 & 1.7 & 0.2388 & 1.6 & 0.93 & 1380.5 & 20.0 & 1396.2 & 13.2 & 1420.3 & 12.4 & 1420.3 & 12.4 \\
\hline 49 & TBP79122-8 & 169 & 171529 & 0.4 & 2.8884 & 1.7 & 0.2332 & 1.7 & 0.97 & 1351.1 & 20.3 & 1378.9 & 13.0 & 1422.2 & 8.5 & 1422.2 & 8.5 \\
\hline 49 & TBP79122-27 & 86 & 60324 & 0.5 & 3.1270 & 1.9 & 0.2513 & 1.4 & 0.72 & 1445.3 & 18.1 & 1439.4 & 14.9 & 1430.5 & 25.6 & 1430.5 & 25.6 \\
\hline 49 & TBP79122-100 & 78 & 111549 & 1.6 & 3.1666 & 2.8 & 0.2540 & 2.6 & 0.92 & 1458.8 & 34.0 & 1449.1 & 21.8 & 1434.7 & 21.0 & 1434.7 & 21.0 \\
\hline 49 & TBP79122-70 & 42 & 117875 & 1.0 & 3.1683 & 2.0 & 0.2535 & 0.7 & 0.33 & 1456.4 & 8.6 & 1449.5 & 15.3 & 1439.3 & 35.6 & 1439.3 & 35.6 \\
\hline 49 & TBP79122-45 & 292 & 519883 & 1.5 & 3.0914 & 0.9 & 0.2466 & 0.9 & 0.97 & 1421.1 & 11.1 & 1430.6 & 6.9 & 1444.6 & 4.2 & 1444.6 & 4.2 \\
\hline 49 & TBP79122-21 & 112 & 77948 & 1.1 & 3.1431 & 2.3 & 0.2507 & 2.1 & 0.93 & 1442.3 & 27.7 & 1443.3 & 17.7 & 1444.7 & 15.8 & 1444.7 & 15.8 \\
\hline 49 & TBP79122-31 & 97 & 76468 & 0.5 & 3.1364 & 1.5 & 0.2499 & 1.2 & 0.78 & 1437.7 & 15.5 & 1441.7 & 11.9 & 1447.5 & 18.3 & 1447.5 & 18.3 \\
\hline 49 & TBP79122-59 & 53 & 113439 & 1.2 & 3.2039 & 1.5 & 0.2529 & 0.9 & 0.59 & 1453.2 & 11.5 & 1458.1 & 11.6 & 1465.3 & 23.2 & 1465.3 & 23.2 \\
\hline 49 & TBP79122-51 & 120 & 93610 & 1.9 & 3.4088 & 4.8 & 0.2662 & 4.7 & 0.99 & 1521.7 & 63.5 & 1506.4 & 37.3 & 1485.1 & 14.9 & 1485.1 & 14.9 \\
\hline 49 & TBP79122-23 & 23 & 30853 & 0.4 & 4.0798 & 4.0 & 0.2901 & 1.4 & 0.36 & 1641.9 & 20.9 & 1650.3 & 32.4 & 1661.0 & 68.5 & 1661.0 & 68.5 \\
\hline 49 & TBP79122-77 & 75 & 102516 & 1.3 & 4.1679 & 1.2 & 0.2947 & 0.7 & 0.58 & 1665.2 & 10.0 & 1667.7 & 9.6 & 1670.9 & 17.7 & 1670.9 & 17.7 \\
\hline 49 & TBP79122-49 & 106 & 147371 & 0.7 & 4.2051 & 0.9 & 0.2973 & 0.7 & 0.74 & 1677.8 & 10.1 & 1675.0 & 7.6 & 1671.5 & 11.6 & 1671.5 & 11.6 \\
\hline 49 & TBP79122-87 & 672 & 459508 & 5.0 & 4.2399 & 0.7 & 0.2991 & 0.7 & 0.98 & 1686.8 & 9.8 & 1681.8 & 5.5 & 1675.5 & 2.4 & 1675.5 & 2.4 \\
\hline 49 & TBP79122-5 & 168 & 360110 & 2.0 & 4.1321 & 4.2 & 0.2908 & 4.2 & 0.99 & 1645.4 & 60.7 & 1660.7 & 34.5 & 1680.0 & 10.8 & 1680.0 & 10.8 \\
\hline 49 & TBP79122-90 & 142 & 137854 & 1.5 & 4.3876 & 2.9 & 0.3083 & 2.9 & 0.97 & 1732.1 & 43.4 & 1710.0 & 24.4 & 1683.0 & 13.4 & 1683.0 & 13.4 \\
\hline 49 & TBP79122-19 & 314 & 324334 & 0.8 & 4.0036 & 1.1 & 0.2809 & 1.0 & 0.98 & 1596.1 & 14.8 & 1634.9 & 8.7 & 1685.2 & 4.3 & 1685.2 & 4.3 \\
\hline 49 & TBP79122-22 & 31 & 33148 & 0.8 & 4.2249 & 2.0 & 0.2964 & 1.0 & 0.49 & 1673.5 & 14.4 & 1678.9 & 16.3 & 1685.6 & 32.0 & 1685.6 & 32.0 \\
\hline 49 & TBP79122-37 & 49 & 41217 & 0.9 & 4.2336 & 2.2 & 0.2967 & 1.9 & 0.86 & 1674.9 & 27.4 & 1680.6 & 17.8 & 1687.6 & 20.4 & 1687.6 & 20.4 \\
\hline 49 & TBP79122-58 & 89 & 126826 & 2.5 & 4.3689 & 3.1 & 0.3060 & 2.9 & 0.93 & 1721.1 & 44.0 & 1706.5 & 25.8 & 1688.5 & 20.9 & 1688.5 & 20.9 \\
\hline 49 & TBP79122-32 & 114 & 162624 & 2.6 & 4.2723 & 1.4 & 0.2989 & 0.9 & 0.65 & 1686.0 & 13.3 & 1688.0 & 11.3 & 1690.6 & 19.3 & 1690.6 & 19.3 \\
\hline 49 & TBP79122-84 & 92 & 172474 & 1.5 & 4.4531 & 1.3 & 0.3112 & 1.2 & 0.90 & 1746.7 & 17.9 & 1722.3 & 10.8 & 1692.7 & 10.3 & 1692.7 & 10.3 \\
\hline 49 & TBP79122-67 & 47 & 108923 & 0.9 & 4.3091 & 4.1 & 0.2999 & 3.9 & 0.94 & 1690.7 & 57.4 & 1695.1 & 33.7 & 1700.6 & 24.7 & 1700.6 & 24.7 \\
\hline 49 & TBP79122-56 & 759 & 1129050 & 2.0 & 4.3353 & 1.5 & 0.3013 & 1.5 & 1.00 & 1697.5 & 23.0 & 1700.1 & 12.7 & 1703.3 & 1.3 & 1703.3 & 1.3 \\
\hline 49 & TBP79122-42 & 119 & 126443 & 1.0 & 4.2295 & 1.0 & 0.2936 & 0.7 & 0.72 & 1659.7 & 10.9 & 1679.8 & 8.5 & 1704.9 & 13.1 & 1704.9 & 13.1 \\
\hline 49 & TBP79122-68 & 136 & 116420 & 3.6 & 4.3164 & 1.0 & 0.2994 & 0.9 & 0.95 & 1688.2 & 13.9 & 1696.5 & 8.1 & 1706.7 & 5.4 & 1706.7 & 5.4 \\
\hline 49 & TBP79122-65 & 94 & 108540 & 0.9 & 4.4419 & 2.9 & 0.3078 & 2.8 & 0.97 & 1729.7 & 42.9 & 1720.2 & 24.1 & 1708.6 & 12.7 & 1708.6 & 12.7 \\
\hline 49 & TBP79122-13 & 60 & 49838 & 1.2 & 4.4479 & 1.6 & 0.3081 & 1.3 & 0.82 & 1731.4 & 19.9 & 1721.3 & 13.3 & 1709.1 & 16.8 & 1709.1 & 16.8 \\
\hline 49 & TBP79122-83 & 42 & 36003 & 1.1 & 4.4454 & 1.8 & 0.3065 & 1.4 & 0.77 & 1723.6 & 21.4 & 1720.8 & 15.1 & 1717.4 & 21.2 & 1717.4 & 21.2 \\
\hline 49 & TBP79122-75 & 159 & 216656 & 2.4 & 4.5027 & 0.8 & 0.3104 & 0.6 & 0.79 & 1742.7 & 9.6 & 1731.5 & 6.6 & 1717.9 & 8.9 & 1717.9 & 8.9 \\
\hline 49 & TBP79122-55 & 135 & 67583 & 1.8 & 4.4827 & 1.8 & 0.3076 & 1.6 & 0.91 & 1728.8 & 24.9 & 1727.8 & 15.0 & 1726.5 & 13.7 & 1726.5 & 13.7 \\
\hline 49 & TBP79122-25 & 346 & 58694 & 2.0 & 4.1266 & 5.1 & 0.2819 & 5.0 & 1.00 & 1601.1 & 71.4 & 1659.6 & 41.3 & 1734.4 & 6.8 & 1734.4 & 6.8 \\
\hline 49 & TBP79122-29 & 231 & 73413 & 1.1 & 4.5324 & 0.8 & 0.3092 & 0.8 & 0.97 & 1736.7 & 12.5 & 1736.9 & 7.0 & 1737.2 & 3.7 & 1737.2 & 3.7 \\
\hline 49 & TBP79122-74 & 321 & 82643 & 3.7 & 4.6030 & 11.6 & 0.3121 & 11.6 & 1.00 & 1750.9 & 178.6 & 1749.8 & 97.4 & 1748.5 & 4.9 & 1748.5 & 4.9 \\
\hline 49 & TBP79122-39 & 212 & 15736 & 1.1 & 4.5721 & 3.2 & 0.3012 & 3.2 & 0.99 & 1697.3 & 47.2 & 1744.2 & 26.5 & 1800.8 & 6.9 & 1800.8 & 6.9 \\
\hline 49 & TBP79122-89 & 152 & 208619 & 2.2 & 5.2414 & 1.5 & 0.3382 & 1.5 & 0.96 & 1878.1 & 24.1 & 1859.4 & 13.2 & 1838.5 & 8.3 & 1838.5 & 8.3 \\
\hline 49 & TBP79122-7 & 82 & 101858 & 0.7 & 5.1102 & 1.1 & 0.3295 & 0.9 & 0.77 & 1835.9 & 13.6 & 1837.8 & 9.5 & 1840.0 & 13.0 & 1840.0 & 13.0 \\
\hline 49 & TBP79122-38 & 152 & 193830 & 1.7 & 5.1965 & 0.9 & 0.3335 & 0.8 & 0.94 & 1855.3 & 13.1 & 1852.0 & 7.3 & 1848.3 & 5.1 & 1848.3 & 5.1 \\
\hline 49 & TBP79122-85 & 653 & 227983 & 3.3 & 4.9329 & 2.8 & 0.3127 & 2.8 & 1.00 & 1753.9 & 42.2 & 1807.9 & 23.3 & 1870.7 & 4.4 & 1870.7 & 4.4 \\
\hline 49 & TBP79122-4 & 222 & 6219 & 1.9 & 10.3473 & 2.1 & 0.4335 & 2.0 & 0.95 & 2321.5 & 39.5 & 2466.3 & 19.8 & 2588.0 & 11.3 & 2588.0 & 11.3 \\
\hline 49 & TBP79122-30 & 22 & 44378 & 0.7 & 11.6851 & 3.3 & 0.4764 & 3.2 & 0.96 & 2511.6 & 66.1 & 2579.5 & 30.9 & 2633.3 & 15.3 & 2633.3 & 15.3 \\
\hline
\end{tabular}


Kimbrough, D.L., Grove, M., Gehrels, G.E., Dorsey, R.J., Howard, K.A., Lovera, O., Aslan, A., House, P.K., and Pearthree, P.A., 2015, Detrital zircon U-Pb provenance of the Colorado River: A 5 m.y. record of incision into cover strata overlying the Colorado Plateau and adjacent regions: Geosphere, v. 11, doi:10.1130/GES00982.1.

TABLE DR8. U-Pb ZIRCON LA-ICP-MS ANALYSES OF SECONDARY ZIRCON STANDARD 49127; 30 MICRON BEAM

\begin{tabular}{|c|c|c|c|}
\hline & & & \\
\hline Analysis & $\begin{array}{c}\text { U } \\
(\mathrm{ppm})\end{array}$ & $\begin{array}{l}206 \mathrm{~Pb} \\
204 \mathrm{~Pb}\end{array}$ & $\mathrm{U} / \mathrm{Th}$ \\
\hline $49127-1$ & 226 & 3947 & 1.0 \\
\hline $49127-2$ & 396 & 6631 & 1.2 \\
\hline $49127-3$ & 231 & 3566 & 0.9 \\
\hline $49127-4$ & 152 & 2912 & 0.8 \\
\hline $49127-5$ & 165 & 3196 & 1.7 \\
\hline $49127-6$ & 224 & 4468 & 1.4 \\
\hline $49127-7$ & 204 & 3535 & 1.0 \\
\hline $49127-8$ & 174 & 3260 & 1.2 \\
\hline $49127-9$ & 182 & 3116 & 0.9 \\
\hline 49127-10 & 217 & 3603 & 0.9 \\
\hline $49127-1$ & 173 & 2882 & 1.1 \\
\hline $49127-2$ & 87 & 1530 & 1.3 \\
\hline $49127-3$ & 211 & 3709 & 1.3 \\
\hline $49127-4$ & 218 & 3709 & 1.1 \\
\hline $49127-5$ & 138 & 2233 & 1.5 \\
\hline $49127-6$ & 142 & 2518 & 0.9 \\
\hline $49127-7$ & 347 & 5939 & 1.2 \\
\hline $49127-8$ & 110 & 1760 & 0.9 \\
\hline $49127-9$ & 157 & 2712 & 0.8 \\
\hline 49127-10 & 272 & 4313 & 1.2 \\
\hline $49127-1$ & 135 & 2276 & 1.6 \\
\hline $49127-2$ & 165 & 2417 & 0.8 \\
\hline $49127-3$ & 226 & 3645 & 1.6 \\
\hline $49127-4$ & 142 & 2429 & 1.2 \\
\hline $49127-5$ & 99 & 1431 & 0.9 \\
\hline $49127-6$ & 143 & 2399 & 0.8 \\
\hline $49127-7$ & 156 & 1693 & 0.8 \\
\hline $49127-8$ & 145 & 1815 & 1.9 \\
\hline 49127-9 & 127 & 2348 & 1.2 \\
\hline 49127-10 & 142 & 2269 & 0.8 \\
\hline 49127-2 & 154 & 2518 & 1.4 \\
\hline $49127-1$ & 190 & 3080 & 1.2 \\
\hline $49127-3$ & 146 & 2480 & 0.8 \\
\hline $49127-4$ & 80 & 1447 & 1.5 \\
\hline $49127-5$ & 338 & 5710 & 1.8 \\
\hline $49127-6$ & 123 & 2176 & 1.0 \\
\hline $49127-7$ & 157 & 2356 & 0.9 \\
\hline $49127-8$ & 119 & 1692 & 0.9 \\
\hline 49127-9 & 328 & 5298 & 1.3 \\
\hline 49127-10 & 138 & 2190 & 1.0 \\
\hline 49127-1 & 200 & 3830 & 1.8 \\
\hline $49127-2$ & 416 & 7255 & 1.2 \\
\hline $49127-3$ & 146 & 2761 & 1.1 \\
\hline $49127-4$ & 127 & 1970 & 1.1 \\
\hline $49127-5$ & 165 & 2408 & 0.8 \\
\hline $49127-6$ & 171 & 1764 & 0.8 \\
\hline $49127-7$ & 68 & 1275 & 1.4 \\
\hline $49127-8$ & 148 & 2452 & 0.8 \\
\hline $49127-9$ & 357 & 6231 & 0.9 \\
\hline $49127-10$ & 111 & 1819 & 1.0 \\
\hline CRTO-806-9-49127-4 & 120 & 1230 & 1.2 \\
\hline RTO-806-9-49127-10 & 313 & 2853 & 2.0 \\
\hline CRTO-806-8-49127-7 & 233 & 3063 & 1.9 \\
\hline CRTO-806-9-49127-5 & 152 & 1174 & 1.0 \\
\hline CRTO-806-8-49127-4 & 244 & 3233 & 1.3 \\
\hline CRTO-806-8-49127-1 & 214 & 2417 & 1.6 \\
\hline CRTO-806-9-49127-6 & 582 & 7176 & 1.6 \\
\hline CRTO-806-8-49127-2 & 113 & 1283 & 1.2 \\
\hline CRTO-806-9-49127-7 & 420 & 4685 & 1.6 \\
\hline CRTO-806-9-49127-9 & 139 & 2171 & 1.1 \\
\hline CRTO-806-8-49127-5 & 184 & 1692 & 1.0 \\
\hline CRTO-806-9-49127-2 & 155 & 2476 & 1.2 \\
\hline CRTO-806-9-49127-8 & 174 & 2819 & 1.0 \\
\hline CRTO-806-8-49127-10 & 131 & 1429 & 1.1 \\
\hline CRTO-806-9-49127-3 & 89 & 2084 & 1.5 \\
\hline CRTO-806-8-49127-8 & 140 & 2371 & 1.0 \\
\hline CRTO-806-9-49127-1 & 418 & 10396 & 1.4 \\
\hline CRTO-806-8-49127-6 & 114 & 2401 & 1.2 \\
\hline CRTO-806-8-49127-9 & 78 & 1221 & 1.6 \\
\hline $06322-6-49127-1$ & 148 & 1720 & 0.5 \\
\hline $06322-6-49127-2$ & 199 & 2988 & 0.7 \\
\hline $06322-6-49127-3$ & 116 & 1224 & 0.5 \\
\hline $06322-6-49127-4$ & 154 & 2594 & 0.9 \\
\hline $06322-6-49127-5$ & 264 & 3776 & 0.8 \\
\hline $32406-1-49127-6$ & 161 & 1452 & 0.5 \\
\hline $32406-1-49127-7$ & 228 & 2609 & 0.5 \\
\hline $32406-1-49127-8$ & 134 & 1458 & 0.6 \\
\hline $32406-1-49127-1$ & 173 & 4089 & 0.7 \\
\hline $32406-1-49127-2$ & 208 & 3138 & 0.5 \\
\hline $32406-1-49127-3$ & 159 & 2877 & 0.5 \\
\hline $32406-1-49127-4$ & 123 & 1612 & 0.7 \\
\hline $32406-1-49127-5$ & 219 & 3111 & 0.7 \\
\hline CR1-49127-1 & 324 & 4416 & 0.5 \\
\hline CR1-49127-10 & 1730 & 20623 & 0.7 \\
\hline CR1-49127-2 & 249 & 2114 & 0.5 \\
\hline CR1-49127-3 & 155 & 2502 & 1.1 \\
\hline CR1-49127-4 & 148 & 2129 & 0.8 \\
\hline CR1-49127-5 & 513 & 6440 & 1.3 \\
\hline CR1-49127-6 & 238 & 2842 & 1.0 \\
\hline CR1-49127-7 & 103 & 1435 & 0.9 \\
\hline CR1-49127-8 & 201 & 2524 & 0.6 \\
\hline CR1-49127-9 & 646 & 10868 & 0.8 \\
\hline ICRTO806-6-49127-1 & 499 & 5878 & 1.1 \\
\hline
\end{tabular}


Kimbrough, D.L., Grove, M., Gehrels, G.E., Dorsey, R.J., Howard, K.A., Lovera, O., Aslan, A., House, P.K., and Pearthree, P.A., 2015, Detrital zircon U-Pb provenance of the Colorado River: A 5 m.y. record of incision into cover strata overlying the Colorado Plateau and adjacent regions: Geosphere, v. 11, doi:10.1130/GES00982.1.

TABLE DR8. U-Pb ZIRCON LA-ICP-MS ANALYSES OF SECONDARY ZIRCON STANDARD 49127; 30 MICRON BEAM

\begin{tabular}{|c|c|}
\hline & \\
\hline Analysis & $\begin{array}{c}U \\
(\mathrm{ppm})\end{array}$ \\
\hline ICRTO806-6-49127-2 & 204 \\
\hline ICRTO806-6-49127-3 & 168 \\
\hline ICRTO806-6-49127-4 & 167 \\
\hline ICRTO806-6-49127-5 & 195 \\
\hline ICRTO806-6-49127-6 & 113 \\
\hline ICRTO806-6-49127-7 & 111 \\
\hline ICRTO806-6-49127-8 & 179 \\
\hline ICRTO806-6-49127-9 & 174 \\
\hline CRTO806-6-49127-10 & 127 \\
\hline CR05-2-49127-3 & 115 \\
\hline CR05-1-49127-5 & 161 \\
\hline CR05-1-49127-2 & 451 \\
\hline CR05-1-49127-1 & 180 \\
\hline CR05-1-49127-4 & 100 \\
\hline CR05-2-49127-1 & 176 \\
\hline CR05-2-49127-7 & 162 \\
\hline CR05-2-49127-4 & 213 \\
\hline CR05-2-49127-6 & 144 \\
\hline CR05-2-49127-8 & 385 \\
\hline CR05-2-49127-2 & 253 \\
\hline CR05-2-49127-5 & 136 \\
\hline CR05-1-49127-3 & 164 \\
\hline $49127-9$ & 480 \\
\hline WICRT08062-49127-5 & 108 \\
\hline WICRT08062-49127-4 & 425 \\
\hline WICRT08062-49127-2 & 271 \\
\hline $49127-10$ & 141 \\
\hline 49127-8 & 199 \\
\hline WICRT08062-49127-1 & 179 \\
\hline WICRT08062-49127-3 & 687 \\
\hline ICRT0806-5-49127-5 & 213 \\
\hline ICRT0806-5-49127-4 & 170 \\
\hline ICRT0806-5-49127-2 & 157 \\
\hline ICRT0806-5-39127-2 & 172 \\
\hline ICRT0806-5-49127-3 & 202 \\
\hline ICRT0806-5-49127-8 & 237 \\
\hline ICRT0806-5-39127-1 & 189 \\
\hline ICRT0806-5-49127-1 & 170 \\
\hline ICRT0806-5-49127-7 & 129 \\
\hline WI06322-37-49127-9 & 171 \\
\hline $06322-37-49127$ & 180 \\
\hline WI06322-37-49127-2 & 159 \\
\hline WI06322-37-49127-8 & 144 \\
\hline W'06322-37-49127-4 & 1548 \\
\hline Wl06322-37-49127-5 & 1334 \\
\hline Wl06322-37-49127-6 & 190 \\
\hline 106322-37-49127-10 & 151 \\
\hline Wl06322-37-49127-7 & 115 \\
\hline W'2-4-06-1-49127-3 & 2671 \\
\hline W'2-4-06-1-49127-7 & 659 \\
\hline W'2-4-06-1-49127-8 & 209 \\
\hline W'2-4-06-1-49127-9 & 117 \\
\hline W'2-4-06-1-49127-6 & 121 \\
\hline |2-4-06-1-49127-10 & 139 \\
\hline W'2-4-06-1-49127-5 & 100 \\
\hline WI2-4-06-1-49127-4 & 285 \\
\hline W'2-4-06-1-49127-2 & 134 \\
\hline FC05-3-49127-5 & 706 \\
\hline FC05-3-49127-3 & 79 \\
\hline FC05-3-49127-7 & 480 \\
\hline FC05-3-49127-8 & 251 \\
\hline FC05-3-49127-4 & 949 \\
\hline FC05-3-49127-2 & 125 \\
\hline FC05-3-49127-9 & 189 \\
\hline FC05-3-49127-1 & 213 \\
\hline FC05-3-49127-6 & 175 \\
\hline 1-22-06-2-49127-2 & 702 \\
\hline 1-22-06-2-49127-7 & 3363 \\
\hline 1-22-06-2-49127-1 & 154 \\
\hline 1-23-06-1-49127-2 & 602 \\
\hline 1-23-06-1-49127-5 & 5411 \\
\hline $1-22-06-2-49127-8$ & 391 \\
\hline 1-23-06-1-49127-6 & 330 \\
\hline $1-22-06-2-49127-4$ & 249 \\
\hline $1-22-06-2-49127-3$ & 307 \\
\hline $1-22-06-2-49127-5$ & 169 \\
\hline FC06-2-49120-1 & 338 \\
\hline FC06-2-49120-3 & 228 \\
\hline FC06-2-49120-4 & 179 \\
\hline FC06-2-49127-6 & 387 \\
\hline FC06-2-49127-8 & 2031 \\
\hline FC06-1-49127-3 & 184 \\
\hline FC06-1-49127-1 & 336 \\
\hline FC06-1-49127-5 & 127 \\
\hline FC06-1-49127-6 & 150 \\
\hline FC06-1-49127-7 & 293 \\
\hline FC06-1-49127-8 & 202 \\
\hline $2-4-06-3-49127-2$ & 169 \\
\hline $2-4-06-3-49127-3$ & 242 \\
\hline $2-4-06-3-49127-4$ & 298 \\
\hline $2-4-06-3-49127-5$ & 215 \\
\hline $2-4-06-3-49127-6$ & 186 \\
\hline $2-4-06-3-49127-7$ & 128 \\
\hline $2-4-06-3-49127-8$ & 292 \\
\hline $2-4-06-3-49127-9$ & 210 \\
\hline
\end{tabular}


Kimbrough, D.L., Grove, M., Gehrels, G.E., Dorsey, R.J., Howard, K.A., Lovera, O., Aslan, A., House, P.K., and Pearthree, P.A., 2015, Detrital zircon U-Pb provenance of the Colorado River: A 5 m.y. record of incision into cover strata overlying the Colorado Plateau and adjacent regions: Geosphere, v. 11, doi:10.1130/GES00982.1.

TABLE DR8. U-Pb ZIRCON LA-ICP-MS ANALYSES OF SECONDARY ZIRCON STANDARD 49127; 30 MICRON BEAM

\begin{tabular}{|c|c|}
\hline & \\
\hline Analysis & $\begin{array}{c}U \\
(\mathrm{ppm})\end{array}$ \\
\hline $2-4-06-3-49127-10$ & 138 \\
\hline 2-4-06-2-49127-11 & 226 \\
\hline $2-4-06-2-49127-12$ & 813 \\
\hline $2-4-06-2-49127-13$ & 213 \\
\hline 2-4-06-2-49127-14 & 692 \\
\hline 2-4-06-2-49127-15 & 183 \\
\hline FC05-149127-1 & 190 \\
\hline FC05-1-49127-2 & 1630 \\
\hline FC05-1-49127-3 & 478 \\
\hline FC05-1-49127-4 & 224 \\
\hline FC05-1-49127-5 & 206 \\
\hline FC05-1-49127-6 & 179 \\
\hline FC05-1-49127-7 & 440 \\
\hline FC05-1-49127-9 & 141 \\
\hline FC05-1-49127-8 & 200 \\
\hline FC05-1-49127-10 & 214 \\
\hline FC05-3-49127-11 & 177 \\
\hline FC05-3-49127-12 & 235 \\
\hline FC05-3-49127-13 & 139 \\
\hline FC05-3-49127-14 & 161 \\
\hline FC05-3-49127-15 & 121 \\
\hline 1-22-06-2-49127-2 & 158 \\
\hline 1-22-06-2-49127-1 & 179 \\
\hline 1-22-06-2-49127-6 & 4015 \\
\hline 1-22-06-2-49127-3 & 127 \\
\hline $1-22-06-2-49127-7$ & 375 \\
\hline $1-22-06-2-49127-8$ & 215 \\
\hline $1-22-06-2-49127-4$ & 152 \\
\hline $1-22-06-2-49127-5$ & 687 \\
\hline CR3-49127-5 & 101 \\
\hline CR2-49127-3 & 88 \\
\hline CR3-49127-7 & 106 \\
\hline CR3-49127-4 & 75 \\
\hline CR2-49127-2 & 45 \\
\hline CR2-49127-5 & 75 \\
\hline CR3-49127-6 & 143 \\
\hline CR2-49127-4 & 68 \\
\hline CR2-49127-6 & 109 \\
\hline CR3-49127-3 & 69 \\
\hline CR3-49127-1 & 92 \\
\hline CR2-49127-1 & 127 \\
\hline CR3-49127-2 & 59 \\
\hline FELIPITO-49127-2 & 116 \\
\hline FELIPITO-49127-4 & 157 \\
\hline 09JBM07-49127-2 & 263 \\
\hline FELIPITO-49127-1 & 1579 \\
\hline FELIPITO-49127-5 & 205 \\
\hline 09JBM07-49127-4 & 192 \\
\hline 09JBM 07-49127-5 & 144 \\
\hline 09JBM 07-49127-1 & 198 \\
\hline FELIPITO-49127-3 & 614 \\
\hline 09JВM07-49127-3 & 285 \\
\hline YUMA-49127-2 & 106 \\
\hline SClara-49127-1 & 166 \\
\hline YUMA-49127-4 & 306 \\
\hline SClara-49127-4 & 190 \\
\hline SClara-49127-5 & 342 \\
\hline YUMA-49127-1 & 113 \\
\hline SClara-49127-3 & 157 \\
\hline YUMA-49127-5 & 130 \\
\hline YUMA-49127-3 & 770 \\
\hline SClara-49127-2 & 953 \\
\hline |32306-175-49127-1 & 324 \\
\hline |32306-175-49127-2 & 534 \\
\hline |32306-175-49127-3 & 677 \\
\hline |32306-175-49127-4 & 453 \\
\hline |32306-175-49127-5 & 107 \\
\hline $32606-49127-6$ & 295 \\
\hline 32606-49127-7 & 81 \\
\hline 32606-49127-8 & 175 \\
\hline $32606-49127-9$ & 85 \\
\hline $32606-49127-10$ & 133 \\
\hline LMSP2-49127-1 & 184 \\
\hline LMSP2-49127-2 & 124 \\
\hline LMSP2-49127-3 & 225 \\
\hline LMSP2-49127-4 & 116 \\
\hline LMSP2-49127-5 & 125 \\
\hline $325061-49127-10$ & 293 \\
\hline $325061-49127-7$ & 102 \\
\hline $325061-49127-8$ & 1739 \\
\hline $325061-49127-9$ & 405 \\
\hline $325061-49127-6$ & 68 \\
\hline CRT0806-49127-1 & 190 \\
\hline CRT0806-49127-2 & 173 \\
\hline CRT0806-49127-3 & 174 \\
\hline CRT0806-49127-4 & 170 \\
\hline CRT0806-49127-5 & 142 \\
\hline CRT0806-49127-6 & 166 \\
\hline CRT0806-49127-7 & 261 \\
\hline CRT0806-49127-8 & 127 \\
\hline CRT0806-49127-9 & 240 \\
\hline WICRT0806-49127-10 & 109 \\
\hline CRT0806-3-49127-11 & 180 \\
\hline CRT0806-3-49127-12 & 5790 \\
\hline CRT0806-3-49127-13 & 343 \\
\hline
\end{tabular}


Kimbrough, D.L., Grove, M., Gehrels, G.E., Dorsey, R.J., Howard, K.A., Lovera, O., Aslan, A., House, P.K., and Pearthree, P.A., 2015, Detrital zircon U-Pb provenance of the Colorado River: A 5 m.y. record of incision into cover strata overlying the Colorado Plateau and adjacent regions: Geosphere, v. 11, doi:10.1130/GES00982.1.

\begin{tabular}{|c|c|c|c|c|c|c|c|c|c|c|c|c|c|c|c|c|c|}
\hline \multirow[b]{2}{*}{ Analysis } & \multirow[b]{2}{*}{$\underset{(\mathrm{ppm})}{U}$} & \multirow[b]{2}{*}{$\begin{array}{l}206 \mathrm{~Pb} \\
204 \mathrm{~Pb}\end{array}$} & & & opic ra & & & & & & arent ages & & & & & & \\
\hline & & & $\mathrm{U} / \mathrm{Th}$ & $\begin{array}{c}207 \mathrm{~Pb}^{*} \\
235 \mathrm{U}\end{array}$ & $\begin{array}{c} \pm \\
(\%)\end{array}$ & $\begin{array}{c}206 \mathrm{~Pb}^{*} \\
238 \mathrm{U}\end{array}$ & $\begin{array}{c} \pm \\
(\%)\end{array}$ & $\begin{array}{l}\text { error } \\
\text { corr. }\end{array}$ & $\begin{array}{c}206 \mathrm{~Pb}^{*} \\
238 \mathrm{U}\end{array}$ & $\begin{array}{c} \pm \\
(\mathrm{Ma})\end{array}$ & $\begin{array}{c}207 \mathrm{~Pb}^{\star} \\
235 \mathrm{U}\end{array}$ & $\begin{array}{c} \pm \\
(\mathrm{M})\end{array}$ & $\begin{array}{l}206 \mathrm{~Pb}^{\star} \\
207 \mathrm{~Pb}^{\star}\end{array}$ & $\begin{array}{c} \pm \\
(\mathrm{Ma})\end{array}$ & $\begin{array}{l}\text { Best age } \\
\text { (Ma) }\end{array}$ & $\begin{array}{c} \pm \\
(\mathrm{Ma})\end{array}$ & $\begin{array}{c}\text { date } \\
\text { analyzed }\end{array}$ \\
\hline CRT0806-3-49127-14 & 319 & 5917 & 1.1 & 0.15224 & 7.0 & 0.02122 & 5.7 & 0.82 & 135.4 & 7.7 & 143.9 & 9.4 & 286.8 & 90.9 & 135.4 & 7.7 & $2006-7$ \\
\hline CRT0806-3-49127-15 & 288 & 2014 & 1.4 & 0.13408 & 4.7 & 0.02081 & 2.7 & 0.57 & 132.8 & 3.5 & 127.8 & 5.6 & 35.4 & 92.1 & 132.8 & 3.5 & $2006-7$ \\
\hline ICRT0806-2-49127-1 & \#DIV/O! & 3433 & \#DIV/O! & 0.15651 & 3.0 & 0.02258 & 1.8 & 0.58 & 144.0 & 2.5 & 147.6 & 4.2 & 207.1 & 57.4 & 144.0 & 2.5 & $2006-7$ \\
\hline CRT0806-2-49127-2 & \#DIVIO! & 1075 & \#DIV/O! & 0.10444 & 13.9 & 0.02241 & 4.2 & 0.31 & 142.9 & 6.0 & 100.9 & 13.3 & -806.4 & 376.6 & 142.9 & 6.0 & $2006-7$ \\
\hline ICRT0806-2-49127-3 & \#DIV/O! & 2103 & \#DIV/O! & 0.11648 & 10.0 & 0.02233 & 2.0 & 0.20 & 142.4 & 2.8 & 111.9 & 10.6 & -497.3 & 260.7 & 142.4 & 2.8 & $2006-7$ \\
\hline CRT0806-2-49127-4 & \#DIV/O! & 3266 & \#DIV/0! & 0.13358 & 6.7 & 0.02188 & 2.2 & 0.32 & 139.5 & 3.0 & 127.3 & 8.0 & -95.6 & 156.0 & 139.5 & 3.0 & $2006-7$ \\
\hline ICRT0806-2-49127-5 & \#DIV/O! & 2990 & \#DIV/O! & 0.13402 & 5.1 & 0.02223 & 1.8 & 0.35 & 141.8 & 2.5 & 127.7 & 6.2 & -126.6 & 119.0 & 141.8 & 2.5 & $2006-7$ \\
\hline ICRT0806-2-49127-1 & 804 & 3433 & 0.9 & 0.15651 & 3.0 & 0.02258 & 1.8 & 0.58 & 144.0 & 2.5 & 147.6 & 4.2 & 207.1 & 57.4 & 144.0 & 2.5 & $2006-7$ \\
\hline ICRT0806-2-49127-2 & 134 & 1075 & 0.9 & 0.10444 & 13.9 & 0.02241 & 4.2 & 0.31 & 142.9 & 6.0 & 100.9 & 13.3 & -806.4 & 376.6 & 142.9 & 6.0 & $2006-7$ \\
\hline ICRT0806-2-49127-3 & 246 & 2103 & 1.1 & 0.11648 & 10.0 & 0.02233 & 2.0 & 0.20 & 142.4 & 2.8 & 111.9 & 10.6 & -497.3 & 260.7 & 142.4 & 2.8 & $2006-7$ \\
\hline ICRT0806-2-49127-4 & 368 & 3266 & 0.6 & 0.13358 & 6.7 & 0.02188 & 2.2 & 0.32 & 139.5 & 3.0 & 127.3 & 8.0 & -95.6 & 156.0 & 139.5 & 3.0 & $2006-7$ \\
\hline ICRT0806-2-49127-5 & 351 & 2990 & 0.6 & 0.13402 & 5.1 & 0.02223 & 1.8 & 0.35 & 141.8 & 2.5 & 127.7 & 6.2 & -126.6 & 119.0 & 141.8 & 2.5 & $2006-7$ \\
\hline NICRT08064-49127-4 & 219 & 1856 & 1.2 & 0.14212 & 12.1 & 0.02072 & 2.2 & 0.18 & 132.2 & 2.9 & 134.9 & 15.3 & 183.1 & 278.9 & 132.2 & 2.9 & $2006-7$ \\
\hline FC063-49127-9 & 1434 & 12936 & 1.8 & 0.15149 & 2.4 & 0.02108 & 1.5 & 0.62 & 134.5 & 2.0 & 143.2 & 3.3 & 290.8 & 43.4 & 134.5 & 2.0 & $2006-7$ \\
\hline FC063-49127-8 & 234 & 2064 & 1.3 & 0.15022 & 9.8 & 0.02123 & 4.4 & 0.45 & 135.4 & 5.9 & 142.1 & 13.0 & 255.0 & 201.6 & 135.4 & 5.9 & $2006-7$ \\
\hline FC063-49127-10 & 541 & 6125 & 1.3 & 0.16045 & 4.0 & 0.02132 & 2.7 & 0.67 & 136.0 & 3.6 & 151.1 & 5.6 & 395.5 & 66.7 & 136.0 & 3.6 & $2006-7$ \\
\hline NICRT08064-49127-3 & 1483 & 9159 & 1.8 & 0.15566 & 2.1 & 0.02150 & 1.2 & 0.57 & 137.1 & 1.7 & 146.9 & 2.9 & 307.9 & 40.1 & 137.1 & 1.7 & $2006-7$ \\
\hline FC063-49127-6 & 504 & 5027 & 1.5 & 0.16818 & 5.6 & 0.02160 & 2.7 & 0.49 & 137.7 & 3.7 & 157.8 & 8.2 & 471.0 & 108.7 & 137.7 & 3.7 & $2006-7$ \\
\hline$=C 063-49127-7$ & 218 & 2394 & 1.2 & 0.15619 & 9.4 & 0.02171 & 2.2 & 0.24 & 138.4 & 3.1 & 147.4 & 12.9 & 293.4 & 208.8 & 138.4 & 3.1 & $2006-7$ \\
\hline WICRT08064-49127-2 & 208 & 1381 & 1.2 & 0.13914 & 11.5 & 0.02171 & 2.6 & 0.23 & 138.5 & 3.6 & 132.3 & 14.3 & 22.7 & 269.7 & 138.5 & 3.6 & $2006-7$ \\
\hline NICRT08064-49127-5 & 221 & 1587 & 1.8 & 0.15081 & 8.9 & 0.02192 & 4.0 & 0.46 & 139.8 & 5.6 & 142.6 & 11.8 & 190.2 & 183.7 & 139.8 & 5.6 & $2006-7$ \\
\hline WICRT08064-49127-1 & 215 & 1635 & 1.2 & 0.14056 & 9.9 & 0.02231 & 2.5 & 0.25 & 142.2 & 3.5 & 133.5 & 12.4 & -18.3 & 233.3 & 142.2 & 3.5 & $2006-7$ \\
\hline CRT080610-49127-4 & 441 & 5077 & 2.1 & 0.14763 & 4.2 & 0.02068 & 2.1 & 0.50 & 132.0 & 2.8 & 139.8 & 5.5 & 275.1 & 83.5 & 132.0 & 2.8 & 2006-7 \\
\hline ICRT080610-49127-5 & 247 & 2157 & 1.3 & 0.14132 & 8.8 & 0.02069 & 3.0 & 0.34 & 132.0 & 4.0 & 134.2 & 11.1 & 173.7 & 193.9 & 132.0 & 4.0 & $2006-7$ \\
\hline CRT080610-49127-9 & 259 & 2438 & 1.2 & 0.13978 & 7.1 & 0.02094 & 1.9 & 0.27 & 133.6 & 2.5 & 132.8 & 8.8 & 119.0 & 161.6 & 133.6 & 2.5 & $2006-7$ \\
\hline CRT080612-49127-11 & 512 & 5837 & 1.4 & 0.15031 & 5.0 & 0.02096 & 3.3 & 0.66 & 133.7 & 4.4 & 142.2 & 6.7 & 286.3 & 86.8 & 133.7 & 4.4 & 2006-7 \\
\hline CRT080610-49127-6 & 109 & 1155 & 1.9 & 0.13927 & 13.2 & 0.02111 & 5.3 & 0.40 & 134.7 & 7.0 & 132.4 & 16.4 & 91.9 & 287.6 & 134.7 & 7.0 & $2006-7$ \\
\hline CRT080612-49127-13 & 197 & 2361 & 1.2 & 0.14838 & 11.0 & 0.02113 & 3.3 & 0.30 & 134.8 & 4.4 & 140.5 & 14.5 & 237.4 & 244.0 & 134.8 & 4.4 & 2006-7 \\
\hline CRT080612-49127-15 & 145 & 2469 & 1.5 & 0.14154 & 9.6 & 0.02121 & 3.8 & 0.39 & 135.3 & 5.1 & 134.4 & 12.0 & 118.8 & 207.6 & 135.3 & 5.1 & 2006-7 \\
\hline CRT080612-49127-12 & 110 & 1136 & 1.4 & 0.13422 & 17.3 & 0.02129 & 4.5 & 0.26 & 135.8 & 6.1 & 127.9 & 20.8 & -17.2 & 406.9 & 135.8 & 6.1 & 2006-7 \\
\hline CRT080610-49127-7 & 10745 & 64017 & 1.6 & 0.14761 & 3.7 & 0.02181 & 3.4 & 0.91 & 139.1 & 4.7 & 139.8 & 4.8 & 152.4 & 35.2 & 139.1 & 4.7 & $2006-7$ \\
\hline ICRT080610-49127-3 & 305 & 4123 & 1.7 & 0.15480 & 6.1 & 0.02196 & 2.7 & 0.44 & 140.0 & 3.7 & 146.1 & 8.3 & 246.9 & 126.2 & 140.0 & 3.7 & $2006-7$ \\
\hline CRT080610-49127-10 & 307 & 4652 & 2.0 & 0.15448 & 8.8 & 0.02238 & 4.6 & 0.52 & 142.7 & 6.4 & 145.9 & 12.0 & 198.1 & 176.2 & 142.7 & 6.4 & $2006-7$ \\
\hline ICRT080610-49127-2 & 539 & 4118 & 1.8 & 0.15317 & 7.6 & 0.02243 & 2.3 & 0.31 & 143.0 & 3.3 & 144.7 & 10.3 & 172.6 & 170.1 & 143.0 & 3.3 & $2006-7$ \\
\hline CRT080612-49127-14 & 146 & 1952 & 1.1 & 0.14338 & 10.7 & 0.02251 & 7.2 & 0.67 & 143.5 & 10.3 & 136.0 & 13.7 & 8.2 & 190.8 & 143.5 & 10.3 & $2006-7$ \\
\hline ICRT080610-49127-8 & 186 & 2378 & 1.1 & 0.16086 & 9.9 & 0.02256 & 3.6 & 0.36 & 143.8 & 5.1 & 151.5 & 13.9 & 273.2 & 212.2 & 143.8 & 5.1 & $2006-7$ \\
\hline CRT080610-49127-1 & 596 & 6794 & 1.5 & 0.16062 & 5.0 & 0.02276 & 3.0 & 0.60 & 145.1 & 4.3 & 151.2 & 7.0 & 249.4 & 91.7 & 145.1 & 4.3 & $2006-7$ \\
\hline ICRT080613-49127-1 & 220 & 2690 & 3.6 & 0.15308 & 10.5 & 0.02056 & 7.6 & 0.72 & 131.2 & 9.8 & 144.6 & 14.1 & 370.8 & 163.1 & 131.2 & 9.8 & $2006-7$ \\
\hline CRT080613-49127-4 & 205 & 2753 & 1.7 & 0.14899 & 13.0 & 0.02068 & 9.5 & 0.73 & 132.0 & 12.5 & 141.0 & 17.1 & 296.2 & 201.2 & 132.0 & 12.5 & $2006-7$ \\
\hline ICRT080613-49127-3 & 203 & 3567 & 1.0 & 0.15356 & 8.1 & 0.02101 & 4.9 & 0.61 & 134.1 & 6.5 & 145.0 & 11.0 & 328.6 & 146.4 & 134.1 & 6.5 & $2006-7$ \\
\hline CRT080613-49127-5 & 97 & 1136 & 1.6 & 0.14002 & $\begin{array}{l}13.3 \\
\end{array}$ & 0.02115 & 3.7 & 0.27 & 134.9 & 4.9 & 133.1 & 16.6 & 100.5 & 304.2 & 134.9 & 4.9 & $2006-7$ \\
\hline ICRT080613-49127-2 & 173 & 1765 & 2.1 & 0.14926 & 9.0 & 0.02159 & 6.8 & 0.76 & 137.7 & 9.3 & 141.3 & 11.9 & 201.5 & 137.6 & 137.7 & 9.3 & $2006-7$ \\
\hline CRT080613-49127-8 & 117 & 1919 & 1.4 & 0.16918 & 8.7 & 0.02027 & 4.4 & 0.50 & 129.4 & 5.6 & 158.7 & 12.8 & 622.8 & 161.8 & 129.4 & 5.6 & $2006-7$ \\
\hline CRT080613-49127-10 & 124 & 2280 & 1.3 & 0.15403 & 7.9 & 0.02041 & 5.4 & 0.69 & 130.3 & 7.0 & 145.5 & 10.6 & 401.2 & 127.1 & 130.3 & 7.0 & $2006-7$ \\
\hline CRT080613-49127-6 & 269 & 4237 & 1.4 & 0.15209 & 8.0 & 0.02064 & 3.2 & 0.40 & 131.7 & 4.1 & 143.8 & 10.7 & 347.2 & 166.6 & 131.7 & 4.1 & $2006-7$ \\
\hline ICRT080613-49127-7 & 155 & 2793 & 1.2 & 0.16286 & 8.2 & 0.02094 & 3.8 & 0.46 & 133.6 & 5.0 & 153.2 & 11.7 & 468.3 & 162.7 & 133.6 & 5.0 & $2006-7$ \\
\hline CRT080613-49127-9 & 3938 & 50472 & 4.9 & 0.14376 & 1.6 & 0.02132 & 1.2 & 0.76 & 136.0 & 1.6 & 136.4 & 2.0 & 143.0 & 23.6 & 136.0 & 1.6 & $2006-7$ \\
\hline DM303-49127-1 & 619 & 4615 & 1.7 & 0.14382 & 2.5 & 0.02065 & 2.0 & 0.81 & 131.8 & 2.7 & 136.4 & 3.2 & 218.1 & 34.5 & 131.8 & 2.7 & $2006-7$ \\
\hline DM303-49127-2 & 398 & 2597 & 1.2 & 0.14412 & 5.1 & 0.02030 & 4.0 & 0.79 & 129.5 & 5.1 & 136.7 & 6.5 & 263.1 & 71.7 & 129.5 & 5.1 & $2006-7$ \\
\hline DM303-49127-3 & 506 & 3937 & 1.9 & 0.14747 & 3.5 & 0.02077 & 2.8 & 0.81 & 132.5 & 3.7 & 139.7 & 4.6 & 263.5 & 46.8 & 132.5 & 3.7 & $2006-7$ \\
\hline DM303-49127-4 & 386 & 2325 & 1.3 & 0.15345 & 3.6 & 0.02079 & 2.7 & 0.75 & 132.7 & 3.5 & 145.0 & 4.8 & 351.0 & 53.3 & 132.7 & 3.5 & $2006-7$ \\
\hline DM303-49127-5 & 331 & 2849 & 1.3 & 0.15143 & 3.6 & 0.02095 & 1.8 & 0.51 & 133.7 & 2.4 & 143.2 & 4.8 & 304.0 & 71.0 & 133.7 & 2.4 & $2006-7$ \\
\hline - $5 \mathrm{HW}-49127-6$ & 173 & 2254 & 1.2 & 0.15160 & 6.5 & 0.02048 & 2.9 & 0.45 & 130.7 & 3.8 & 143.3 & 8.7 & 357.8 & 132.2 & 130.7 & 3.8 & $2006-7$ \\
\hline H5HW-49127-7 & 154 & 1543 & 1.5 & 0.15757 & 7.2 & 0.02038 & 3.2 & 0.44 & 130.1 & 4.1 & 148.6 & 10.0 & 454.8 & 144.6 & 130.1 & 4.1 & $2006-7$ \\
\hline $45 \mathrm{HW}-49127-8$ & 157 & 1570 & 1.2 & 0.14638 & 6.6 & 0.02038 & 2.1 & 0.33 & 130.1 & 2.8 & 138.7 & 8.5 & 288.9 & 142.1 & 130.1 & 2.8 & $2006-7$ \\
\hline H5HW-49127-9 & 207 & 1904 & 1.2 & 0.14854 & 6.6 & 0.02041 & 3.1 & 0.48 & 130.2 & 4.0 & 140.6 & 8.6 & 319.7 & 131.6 & 130.2 & 4.0 & $2006-7$ \\
\hline H5HW-49127-10 & 168 & 1729 & 1.1 & 0.14588 & 4.4 & 0.01990 & 2.1 & 0.48 & 127.0 & 2.7 & 138.3 & 5.7 & 336.4 & 86.7 & 127.0 & 2.7 & $2006-7$ \\
\hline PARKER3-49127-4 & 224 & 3030 & 1.5 & 0.16057 & 4.9 & 0.02060 & 2.3 & 0.46 & 131.5 & 2.9 & 151.2 & 6.9 & 473.1 & 97.4 & 131.5 & 2.9 & $2006-7$ \\
\hline PARKER3-49127-5 & 87 & 1299 & 1.8 & 0.15573 & 8.5 & 0.02063 & 2.9 & 0.34 & 131.7 & 3.7 & 147.0 & 11.6 & 401.5 & 178.7 & 131.7 & 3.7 & $2006-7$ \\
\hline PARKER3-49127-1 & 115 & 2168 & 1.3 & 0.15944 & 7.5 & 0.02070 & 2.6 & 0.35 & 132.1 & 3.5 & 150.2 & 10.5 & 446.6 & 157.0 & 132.1 & 3.5 & $2006-7$ \\
\hline PARKER3-49127-3 & 159 & 2904 & 1.2 & 0.16197 & 3.4 & 0.02080 & 1.9 & 0.57 & 132.7 & 2.5 & 152.4 & 4.8 & 471.1 & 61.3 & 132.7 & 2.5 & $2006-7$ \\
\hline PARKER3-49127-2 & 128 & 2459 & 1.9 & 0.17029 & 4.8 & 0.02098 & 2.4 & 0.49 & 133.9 & 3.2 & 159.7 & 7.2 & 562.0 & 91.7 & 133.9 & 3.2 & $2006-7$ \\
\hline $325063-49127-6$ & 193 & 2483 & 1.3 & 0.14814 & 3.8 & 0.02020 & 1.6 & 0.41 & 128.9 & 2.0 & 140.3 & 5.0 & 336.7 & 78.0 & 128.9 & 2.0 & $2006-7$ \\
\hline 325063-49127-7 & 226 & 2942 & 1.0 & 0.15076 & 3.2 & 0.02027 & 1.2 & 0.36 & 129.4 & 1.5 & 142.6 & 4.2 & 368.8 & 66.7 & 129.4 & 1.5 & $2006-7$ \\
\hline $325063-49127-8$ & 157 & 1783 & 0.9 & 0.15795 & 5.6 & 0.02025 & 2.8 & 0.50 & 129.2 & 3.5 & 148.9 & 7.7 & 475.3 & 106.8 & 129.2 & 3.5 & $2006-7$ \\
\hline $325063-49127-9$ & 74 & 1169 & 1.5 & 0.16944 & 9.6 & 0.02074 & 3.1 & 0.33 & 132.3 & 4.1 & 158.9 & 14.1 & 576.5 & 197.4 & 132.3 & 4.1 & $2006-7$ \\
\hline 325063-49127-10 & 167 & 1989 & 1.0 & 0.14906 & 4.8 & 0.02029 & 2.4 & 0.49 & 129.5 & 3.1 & 141.1 & 6.4 & 340.3 & 95.5 & 129.5 & 3.1 & $2006-7$ \\
\hline H5HW21-49127-1 <> & 129 & 8851 & 1.3 & 0.1280 & 16.5 & 0.0206 & 3.7 & 0.22 & 131.6 & 4.8 & 122.3 & 19.1 & -55.8 & 395.0 & 131.6 & 4.8 & 2013-11 \\
\hline H5HW21-49127-2 <> & 181 & 16194 & 1.1 & 0.1237 & 11.4 & 0.0210 & 1.5 & 0.13 & 134.1 & 2.0 & 118.4 & 12.7 & -187.7 & 282.6 & 134.1 & 2.0 & $2013-11$ \\
\hline H5HW21-49127-3<> & 166 & 13434 & 0.9 & 0.1445 & 12.9 & 0.0210 & 1.2 & 0.09 & 134.2 & 1.6 & 137.0 & 16.5 & 187.2 & 300.1 & 134.2 & 1.6 & 2013-11 \\
\hline $34942-49127-1<>$ & 96 & 9313 & 1.0 & 0.1019 & 28.2 & 0.0210 & 5.7 & 0.20 & 133.9 & 7.5 & 98.6 & 26.5 & -690.3 & 777.4 & 133.9 & 7.5 & 2013-11 \\
\hline $34942-49127-2<>$ & 263 & 11570 & 1.0 & 0.1437 & 13.1 & 0.0211 & 1.7 & 0.13 & 134.9 & 2.3 & 136.3 & 16.7 & 161.1 & 303.8 & 134.9 & 2.3 & 2013-11 \\
\hline $34942-49127-3<>$ & 146 & 9172 & 0.9 & 0.1454 & 11.7 & 0.0214 & 3.1 & 0.26 & 136.4 & 4.2 & 137.8 & 15.1 & 162.5 & 265.3 & 136.4 & 4.2 & 2013-11 \\
\hline
\end{tabular}


Kimbrough, D.L., Grove, M., Gehrels, G.E., Dorsey, R.J., Howard, K.A., Lovera, O., Aslan, A., House, P.K., and Pearthree, P.A., 2015, Detrital zircon U-Pb provenance of the Colorado River: A 5 m.y. record of incision into cover strata overlying the Colorado Plateau and adjacent regions: Geosphere, v. 11, doi:10.1130/GES00982.1.

TABLE DR9. REPLICATE ANALYSES OF NEW ZEALAND GABBRONORITE OU49127

\begin{tabular}{|c|c|c|c|c|c|c|c|c|c|}
\hline date & fraction & *method & wt (mg) & U ppm & $206 / 204$ & $206 / 238$ & $207 / 235$ & $207 / 206$ & $7 / 6 \mathrm{err}$ \\
\hline Apr-84 & coarse & \&AVCO & 9.7 & 308.4 & 3077 & 136.58 & 136.76 & 139.8 & 4.0 \\
\hline Apr-84 & fine & \&AVCO & 11.8 & 434.4 & 3021 & 136.51 & 136.67 & 139.4 & 4.0 \\
\hline Dec-91 & bulk & \#VG (HF) & 4.0 & 322.6 & 30451 & 137.13 & 137.30 & 140.3 & 0.5 \\
\hline Dec-91 & bulk & \#VG (HF) & 3.2 & 369.3 & 21657 & 137.45 & 137.62 & 140.7 & 0.8 \\
\hline Dec-91 & bulk & \#VG (HF) & 3.0 & 325.6 & 3438 & 137.29 & 137.61 & 143.2 & 2.2 \\
\hline Apr-92 & bulk & VG & 3.3 & 300.9 & 1547 & 136.84 & 136.76 & 135.4 & 4.0 \\
\hline Sep-92 & bulk & VG & 6.6 & 274.3 & 2355 & 136.77 & 136.94 & 139.9 & 1.8 \\
\hline Mar-93 & bulk & VG UO2 & 4.2 & 250.6 & 2479 & 136.69 & 136.97 & 141.9 & 2.9 \\
\hline Mar-93 & bulk & VG UO2 & 4.2 & 236.4 & 1901 & 136.67 & 136.84 & 139.8 & 3.3 \\
\hline Mar-93 & bulk & VG UO2 & 3.9 & 302.2 & 2269 & 136.68 & 136.90 & 140.8 & 3.6 \\
\hline Mar-93 & bulk & VG UO2 & 4.3 & 193 & 2771 & 136.74 & 136.66 & 135.3 & 2.7 \\
\hline Mar-93 & bulk & VG UO2 & 4.6 & 272.3 & 2905 & 136.47 & 136.61 & 139.1 & 2.4 \\
\hline Mar-93 & bulk & VG UO2 & 4.4 & 279.7 & 2579 & 136.64 & 136.82 & 139.9 & 3.4 \\
\hline Dec-93 & bulk & VG UO2 & 1.3 & 133.1 & 2514 & 137.17 & 137.16 & 137.0 & 1.3 \\
\hline Dec-93 & bulk & VG UO2 & 1.0 & 144.8 & 2905 & 137.27 & 137.33 & 138.3 & 1.6 \\
\hline Apr-94 & bulk & VG UO2 & 1.2 & 305 & 3268 & 136.81 & 136.71 & 135.0 & 4.3 \\
\hline \multirow[t]{3}{*}{ Apr-94 } & bulk & VG UO2 & 0.6 & 169.3 & 2239 & 137.47 & 137.66 & 141.9 & 1.7 \\
\hline & & & & AVERAGE & & 136.89 & 137.02 & 139.3 & 2.6 \\
\hline & & & & STD DEV & & 0.33 & 0.36 & 2.39 & 1.20 \\
\hline
\end{tabular}

${ }^{*}$ All analyses by Thermal Ionisation Mass Spectrometry

AVCO = mass spectrometry on the UC Santa Barbara single collector AVCO instrument

VG = mass spectrometry on the SDSU VG Sector 54 multicollector instrument

$\mathrm{HF}=$ leaching of sample in $\mathrm{HF}+\mathrm{HNO} 3$ on hotplate overnight prior to sample digestion

$\mathrm{UO} 2$ = uranium run as oxide loaded onto $\mathrm{Pb}$ ID run

for sample analysis details see Kimbrough et al. (1992)

$\&=$ analyses published in Mattinson et al. (1986)

\# = analyses published in Kimbrough et al. (1994) 